\title{
Freedom and Uncertainty:
}

\section{Contemporary liberal theory examined}

from the perspective of moral uncertainty

A thesis submitted in partial fulfilment of the requirements for the

degree of Doctor of Philosophy in Law

in the University of Canterbury

by Matthew Barber

University of Canterbury

2008 


\section{TABLE OF CONTENTS}

\section{Introduction}

1. Moral uncertainty

1.1 Protagoras and relativism 7

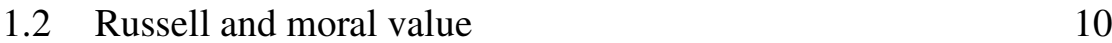

1.3 The postmodern condition 13

2. Liberal theory 16

3. Method 18

3.1 The perspective of moral uncertainty 18

$\begin{array}{ll}3.2 \text { Liberalism examined } & 20\end{array}$

3.3 Summary of thesis 22

2. A Theory of Justice

1. Introduction 24

2. Basic structure $\quad 27$

2.1 Approach to justification 27

2.2 Role of the original position 28

$\begin{array}{ll}2.3 \text { Conditions of the original position } & 30\end{array}$

2.4 Outcome of the original position 32

3. Analysis 35

3.1 Universal aims 35

3.2 Justification and the original position 37

3.3 Moral intuitions and considered judgments $\quad 40$

3.4 Deontology, the right and the good 45

3.5 Implied conceptions $\quad 51$

3.6 Two principles of justice $\quad 59$

4. Conclusion 66

3. Anarchy, State, and Utopia 70

1. Introduction $\quad 70$

2. Basic structure $\quad 72$

$\begin{array}{lll}2.1 & \text { Structure and justification } & 72\end{array}$

2.2 The state of nature and the ultraminimal state 75

2.3 The minimal state $\quad 77$

2.4 Beyond the minimal state $\quad 82$

3. Analysis 86

3.1 Universal aims and justificatory approach $\quad 86$

$\begin{array}{lr}3.2 \text { Natural rights } & 90\end{array}$

3.3 The ultraminimal state 101

3.4 The minimal state 103

3.5 Beyond the minimal state 108

4. Conclusion 110 
4. Equality and neutrality 114

1. Introduction 114

2. Dworkin and equality 114

2.1 Equal concern and respect $\quad 115$

2.2 Distributive equality 118

2.3 Liberalism and equality 126

2.4 Equality revisited 138

3. Ackerman and neutrality 142

3.1 Neutral liberal dialogue 142

3.2 Neutrality and equality 146

3.3 Diversity, exchange and justification $\quad 150$

4. Contemporary liberal theory 154

5. Pluralism 158

1. The communitarian critique of liberalism 158

1.1 The unencumbered self 158

1.2 The enlightenment critique 162

2. Liberal responses 167

2.1 Pluralism in liberal theory 167

2.2 Walzer's complex equality 168

$\begin{array}{lll}2.3 & \text { Raz's perfectionism } & 175\end{array}$

$\begin{array}{ll}2.4 \text { Gray's post-liberalism } & 185\end{array}$

3. Liberal pluralism 193

3.1 Contextual justification 194

3.2 Conceiving pluralism 196

3.3 Pluralism and liberalism 198

3.4 From pluralism to liberalism 203

3.5 Galston's Liberal Pluralism 207

4. Conclusion 213

6. Political liberalism and public reason 219

1. The political and the metaphysical 219

2. Political Liberalism 221

2.1 The political and the reasonable 221

2.2 The political and the true 226

2.3 Reasonable pluralism 230

2.4 From reasonable pluralism to political liberalism 235

2.5 Overlapping consensus 240

2.6 Public reason 242

2.7 Conclusion 247

3. Patterns of Moral Complexity 250

3.1 Neutral political neutrality 254

3.2 Larmore's public reason $\quad 257$

4. Liberal public reason $\quad 259$

4.1 Habermas and discourse ethics 259

4.2 Deliberative democracy 261

4.3 Justificatory liberalism 268

4.4 Public reason in liberalism 281 
7. Multiculturalism 287

1. Liberal theory and group rights 287

$\begin{array}{ll}1.1 & \text { The multicultural movement } \\ 1.2 & 287\end{array}$

1.2 The politics of recognition 288

2. Multicultural citizenship 293

2.1 Group rights as liberal rights 293

2.2 Culture and multiculturalism 299

2.3 Societal culture and liberal freedom 303

2.4 Minority self-government and individual rights 305

2.5 Culture and Equality 308

2.6 Liberal bias and equal treatment 311

2.7 Liberalism and multiculturalism $\quad 315$

3. Multiculturalism beyond liberalism 318

3.1 Demands for recognition 318

3.2 Cultural understandings of culture 324

3.3 Rethinking Multiculturalism 326

3.4 Multicultural attitudes to liberalism 332

4. Conclusion 337

8. Postmodernism 342

1. Rorty's postmodern liberalism 342

1.1 Contingency and reality 343

1.2 Postmodernism and theory 346

1.3 Contingency and liberalism 347

2. Analysis 351

2.1 Theory and contingency 351

2.2 Justification and liberalism 356

2.3 Irony and solidarity 361

2.4 Liberal ironism 365

3. Conclusion 368

9. Freedom and uncertainty 373

1. Contemporary liberal theory 373

1.1 Approach of thesis 373

1.1 Early contemporary liberal theory 375

1.2 Post-communitarian liberal theory 380

2. Responding to moral uncertainty 389

2.1 Liberal duality $\quad 389$

2.2 Intuitions in liberal theory 391

2.3 Biased conceptions of liberalism 393

3. Developing liberal theory $\quad 395$

3.1 The problem with liberal theory $\quad 395$

3.2 Liberal theory and value 395

3.3 A framework for liberal theories $\quad 400$

3.4 Beyond the framework 402

3.5 Some objections 408

3.6 Concluding remarks 410

$\begin{array}{ll}\text { References } & 413\end{array}$ 


\section{Table of graphs}

1. Resource-Equalising Insurance Market

131

2. $50 \%$ Income Coverage

132

3. $50 \%$ Income Coverage (2)

133

4. Maximal Insurance

134 


\section{Abstract}

This thesis aims to use general assertions of moral uncertainty as a perspective by which to explore and illuminate contemporary strands of liberal theory. It examines the work of the earlier contemporary liberal theorists, including John Rawls, Robert Nozick, Ronald Dworkin and Bruce Ackerman, as well as the more recent accounts of liberalism that express ideas of pluralism (Michael Walzer, Joseph Raz, John Gray, William Galston, George Crowder), political liberalism (John Rawls, Charles Larmore), public reason (Amy Gutmann \& Dennis Thompson, Gerald Gaus), multiculturalism (Charles Taylor, Will Kymlicka, Brian Barry, James Tully, and Bikhu Parekh), and postmodernism (Richard Rorty). The development from the earlier to more recent liberal theories represents a fundamental shift in justificatory strategy: where earlier liberal conceptions aim at universality, and at overcoming or transcending uncertainty, later approaches make this uncertainty, usually in the form of pluralism or difference, central to the liberal project. In order to achieve this, these latter theories tended to presuppose the circumstances of western society, or western democratic values. Generally speaking, these approaches fail to respond adequately to moral uncertainty, and to meet their own justificatory aims. This manifests, in the earlier theories, as plausible but contestable central conceptions, and, in the more recent theories, as the inability to justify particular liberal conceptions in the face of persistence difference. This is an important result, and suggests the need for further developments in liberal justificatory strategies. I suggest that one viable approach would be for liberal theory to accept moral uncertainty, and work from a model of society and self towards a more successful liberal conception.

\section{Acknowledgements}

I would like to acknowledge the assistance and support of Geoff Leane, Professor Mark Francis, and John Hopkins, as well as the tireless proof-reading efforts of Kate Henson. 


\section{INTRODUCTION}

\section{Moral uncertainty}

\subsection{Protagoras and relativism}

The concept of moral uncertainty represents a number of ideas about and perspectives on moral theory. These vary in meaning and expression, and are potentially quite diverse, but what is common between them is the doubt they express as to the ability of a moral theory to provide a set of positive and absolute guidelines for action that are universal, knowable and expressible. This incorporates the group of ideas ascribed such names as relativism, subjectivism and scepticism. This doubt acts not only to criticise the grand moral theories that purport to set out some rationally incontestable truth, but also promotes alternative approaches to working out how we should act. These sorts of answers face considerable difficulties: having criticised absolutism in the moral theory of others, the challenge is then to arrive at some definite and useful result that does not suffer from the same ailment.

One of the earliest proponents of this kind of moral uncertainty in the western tradition was Protagoras. He was the most successful member of the Sophists, a group of professional philosophers and teachers who lived in Greece in fifth century BC. ${ }^{1}$ He was a contemporary of Socrates, and is best known for featuring in two Platonic dialogues, Protagoras and Theaetetus. He is famous for his oft-quotes assertion that: "Man is the measure of all things, of things that are as to how they are, and of things that are not as to how they are not."2 This statement has been subject to a number of interpretations. In a relatively weak rendering, it can be understood as meaning that the characteristics of a thing depend on the perspective of each person; or in other words "if honey seems sweet to some and bitter to others, then it is sweet to those to

\footnotetext{
${ }^{1}$ T Honderich (ed.), The Oxford Companion to Philosophy (1995, p725

${ }^{2}$ G B Kerferd, The Sophistic Movement (1981), p85.
} 
whom it seems sweet, and bitter to those to whom it seems bitter". ${ }^{3}$ This is contrary to the more natural idea, that the honey is sweet regardless of who tastes it, and, so, is universally sweet. It seems, then, that Protagoras is saying more than simply that different people perceive things in different ways; rather, he is asserting that the natures of the things themselves are dependant upon these individual perceptions. To recapitulate this argument: since perceptions vary, the natures of things also vary, and are dependant on the identity and position of the person.

This idea has broad and important implications, especially if the subjectivity of the characteristics of objects is extended to encompass the properties and nature of any moral situation or action. This would suggest that the correct moral analysis of a situation is dependant on the position, the context and beliefs, of the person sitting in judgment. This result is both important and startling, and it threatens to undermine attempts to apply any moral judgment generally, and, by extension, any judgment at all. The dramatic result of Protagoras' arguments is that

[t]ruth [is] relative, not absolute, and differ[s] from culture to culture, from person to person, and from situation to situation. Claims to the contrary, whether religious or philosophical, [can]not stand up to critical argument. The ultimate value of any belief or opinion [can] be judged only by its practical utility in serving the individual's needs in life. ${ }^{4}$

This position is sceptical of any moral theory based on a universal view of nature or reason, and leads to a number of theoretical difficulties. Probably the most striking of these is the argument of Plato that such a position is self-refuting: if all perceptions are true, then it is never possible that they be corrected or disproved, even by the same person at a later time. ${ }^{5}$ If this is extended to beliefs generally, then all beliefs must be true, including the belief that not all beliefs are true. ${ }^{6}$

Neither Protagoras' view nor Plato's criticism of it have been expressed here sufficiently so as to allow the validity of either to be rigorously explored; but it does seem that Plato has identified a fundamental difficultly that could apply to any argument critical of absoluteness or universality. This difficulty is characterised by

\footnotetext{
3 Ibid p86.

${ }^{4} \mathrm{R}$ Tarnas, The Passion of the Western Mind: Understanding the Ideas That Have Shaped Our World View 1996), p27.

${ }^{5}$ Kerferd, op cit, above note 2, p88.

${ }^{6}$ Honderich, op cit, above note $1, \mathrm{p} 725$.
} 
the contradiction between an argument against absolutism and the absolute way in which that argument is made. In relation to this example, Protagoras' argument against absolute judgment leads ultimately to the position that all beliefs are true. This, however, is contradicted by the (consequently true) belief to the contrary. By being applied universally, the idea that meaning is dependant on perception undermines itself; the relativistic consequences of the idea cannot sit easily with the absolute manner in which it is applied.

Someone supporting Protagoras' basic claim might argue against this conclusion. They might criticise the way that the 'man as the measure' idea has been extended so easily, and without qualification, to all beliefs. They might also argue that the beliefs of individuals are not 'true' in the same way as the more fundamental or general truth about beliefs; in other words, that the statement about the truth of all claims is a rule that defines how the system of beliefs works, while the actual beliefs of the individuals themselves are the workings of that system. Individuals, then, can believe that not all beliefs are true, and this belief can itself be true, within the bounds of that system.

This sort of argument initially seems quite plausible. It allows that individuals have their own incontestable beliefs, yet it also allows some general statements to be made about the nature or structure of these beliefs. It simply asserts that these two operate within different spheres, so that their truth is not comparable. On further reflection, however, this position actually undermines the initial claim Protagoras was making, that truths are not universal, but relative, and, so, incapable of being disputed. This reply to Plato's criticism sets up a type of truth that is capable of being universal and absolute, and then argues that the 'man as a measure' premise is one of these truths.

In beginning to characterise the position of moral uncertainty, then, two fundamental problems can be recognised. The first refers to the approach of undermining any absolute claims in a manner that is itself absolute, or, in other words, being sceptical, but being certain in the manner of that scepticism. This is the sense in which such scepticism is self-refuting, and there is no obvious way to overcome this. When this approach is extended to assert some substantive moral theory, the second problem becomes apparent. Since few theorists are willing to embrace a moral free-for-all 
where no meaningful judgment can be made about any action, they need to overcome their own sceptical approach and espouse a substantive moral position that is, in some sense, universal. The difficulty lies in achieving this without falling subject to the very criticism they have levelled at more ostensibly absolute theories.

These two problems are closely related, and must be addressed by any sceptical moral theory. Consequently, if liberal theories are to adopt a position that recognises or adopts moral uncertainty, they must in some way overcome these problems. The possible approaches to achieving this vary, depending on the theories themselves. Not all moral criticisms are expressions of the same formulation set out by Protagoras.

\title{
1.2 Russell and moral value
}

An alternative approach to moral uncertainty can be found in the work of Bertrand Russell. In Religion and Science, he maps the historical conflicts between natural science and religion, and then uses these to build an understanding of the nature of moral value. He determines that rational and universal conceptions of the meaning of good and bad are always flawed:

\begin{abstract}
questions as to "values" lie wholly outside the domain of knowledge. That is to say, when we assume that this or that has "value," we are giving expressions to our own emotions, not to a fact which would still be true if our personal feelings were different.... Ethics is an attempt though not, I think a successful one - to escape from this subjectivity. ${ }^{7}$
\end{abstract}

In this way, moral value cannot be knowable in the sense of this representing an objective truth. In addition, moral judgments are merely personal preferences, and are not congruent between people, so that "what one man calls 'sin' another may call 'virtue,' and though they may dislike each other on account of this difference, neither can convict the other of intellectual error." 8

This view is implicitly critical of any moral theory that claims to be universal or objective. Such theories assert that a particular moral judgment can be identified that

\footnotetext{
${ }^{7}$ Russell, B A W, Religion and Science (1997), pp230-231.

${ }^{8}$ Ibid, pp238-239.
} 
is potentially recognisable by every person, and that such a judgment ought to be agreed with and followed by all. With Russell's approach, each judgment is inherently personal; no general statement can be made about any judgment that transcends individual or group perception. In this sense he agrees with Protagoras that the perspective of each person is distinct and unable to be corrected or disproved.

This is not to say that the views of the two are the same, however. Russell's focus is not on the nature of individual perceptions and the characteristics of objects, but, rather, on the evaluation of the meaning of moral value. Protagoras asserts that moral (and, potentially, all) judgment is constructed by the individual who perceives the situation. An objective perspective is impossible since the nature of that which is being judged is dependant on the individual who judges it. In contrast, Russell says that we can judge a moral theory by its consistency with our own feelings, and can then determine whether or not to like it. This suggests that the nature of morality is such that no independent criteria can be applied to determine its universal value. An objective perspective is impossible because moral judgments can never have any meaning beyond the feelings or intuitions of the person or group perceiving them.

Russell's comments are narrower than those of Protagoras in that they apply only to moral theory. This approach means that an answer to the first of the two problems of moral uncertainty is more easily forthcoming. Because his assertion applies only to morality, he is capable of making general comments about moral judgments that are not self-refuting, since those comments are not themselves moral judgments. For this approach to be convincing, the actual nature of morality would have to be explicitly described and explored in such a way as to distinguish it from other spheres of knowledge or judgment that might themselves allow such comparison, e.g. scientific knowledge. This would involve overcoming the plausible view that meaningful comments can be made about the moral judgments of others, beyond a mere expression of dislike. While these tasks would not be easy, they suggest a method by which the first problem can be convincingly overcome.

The second problem raises more difficulties in relation to Russell's approach. If all we can say about the moral judgments of others is that we like or dislike them, then there is no independent way of choosing between alternative judgments or theories. The 
only criteria we can apply are the feelings of the target individual or group. The result, that there is no means of justifying, or disapproving of, an ethical position, is quite disconcerting and, to many, would be deeply troubling. The attempt might be made to avoid this result by introducing some external criterion that acts as an independent measure. One approach would be to give some value to the common view of acceptable morality within a society or culture. This would mean championing the boundaries of common moral judgment, not as the correct ones, but as ones that we should, in the circumstances, follow.

This idea breaks down once the argument is fully expanded. For it to succeed there would need to be some valid justification for promoting the common judgment. To achieve this, a single factor might be identified, say stability, that tends to be improved or maximised when the common judgment is accepted. The problem is that the decision to follow the stable judgment is itself a moral judgment, and, so, subject to Russell's original criticism; consequently, we cannot comment on it other than to state our like or dislike for it. To say that any criterion should guide our moral judgment amounts to making a statement that is itself of a moral nature, and is morally contestable. Russell leaves us little way out of this, and little possibility of talking sensibly or rationally about our personal or group morals. The point could be made that our natures and upbringings do in fact predispose us to follow the norms and morals of the culture in which we live, but this in no way justifies the views of that culture, nor can it act to condemn the person whose views are in violent opposition. Although we might identify an actual moral status quo, moral theory has no power to comment on or validate this.

Of course, the positions of both Protagoras and Russell have been described here in only the most superficial manner. Further enquiry would reveal new depths and complexities, which would lead to new discussions and arguments. The purpose of citing these philosophers, then, is not to fully explore their work, but, rather, to begin to characterise the nature of moral uncertainty. In making the comparison between Protagoras and Russell, what should be apparent are the variations in approach, both obvious and subtle, but also the common thread, that each is sceptical towards absolutism and objectivity in moral judgment and theory. 


\subsection{The postmodern condition}

This comparison can be extended by including a more contemporary view of moral uncertainty, and one that will be revisited in the context of recent liberal theory, namely postmodernism. It is difficult to attach any definite content to the postmodern movement; the adjective 'postmodern' can offer many different meanings, and the movement itself is diverse, both in understanding and in scope. ${ }^{9}$ Perhaps the most familiar attempt to characterise the postmodern was made by Jean-François Lyotard in his book The Postmodern Condition: "Simplifying to the extreme, I define postmodern as incredulity toward metanarratives." 10 These metanarratives are the "supposedly transcendent and universal truths that underpin western civilization and that function to give that civilization objective legitimation". ${ }^{11}$ Lyotard's postmodernism, then, involves scepticism towards the grand theories and truths of modernity, including the purportedly objective application of rationality to problems in order to achieve universal solutions. This general approach leads to a number of ideas:

There is an appreciation of the plasticity and constant change of reality and knowledge, a stress on the priority of concrete experience over fixed abstract principles, and a conviction that no single a priori thought system should govern belief or investigation.... The critical search for truth is constrained to be tolerant of ambiguity and pluralism, and its outcome will necessarily be knowledge that is relative and fallible rather than absolute or certain. ${ }^{12}$

Contracting the postmodern condition involves recognising the 'objective truths' for what they are, namely the subjective traditions of some group, culture or discipline. In addition to this recognition, postmodernism may also prescribe an appropriate response. This can involve an attitude of self-awareness or irony, and the acceptance of the validity of a pervasive value-pluralism. In the words of Lyotard, postmodern knowledge "refines our sensitivity to differences and reinforces our ability to tolerate the incommensurable". ${ }^{13}$ It is in this sense that postmodernism goes beyond criticism and achieves its own approach, attitude or, perhaps, style. A style that, being

\footnotetext{
${ }^{9}$ T Mautner, The Penguin Dictionary of Philosophy (2000), p439; Honderich, op cit, above note 1, p708.

${ }^{10}$ J F Lyotard, The Postmodern Condition: a report on knowledge (1984), pxxiv.

${ }^{11} \mathrm{~J}$ W Bertens, The Idea of the Postmodern: A History (1995), p124.

${ }^{12}$ Tarnas, op cit, above note 4, pp395-396.

${ }^{13}$ Lyotard, op cit, above note 10, pxxv.
} 
necessarily divorced from usual methodologies and constraints, claims to be illuminating and liberating, yet often seems difficult, unapproachable and devoid of meaning.

The approach of postmodernism can be applied to morality and moral theory. This would involve recognising that the inherited moral theories and judgments of western society posit a false objectivity, and an invalid process of legitimation. Instead of blind adherence to these judgments, one should be aware of their limitations, and of the potential for valid alternatives. Although the underlying message of this criticism is one that is, in some sense, shared by both Protagoras and Russell, there are obvious differences in focus and approach. Lyotard is concerned with questioning western beliefs and perspectives. This shares with Protagoras a belief in both the subjectivity and constructedness of moral judgment, but is concerned with the historical development and ossification of traditions and truths and their lack of true validity, rather than the necessarily individualistic nature of judgment. A deeper inquiry into postmodernism would reveal additional differences in focus, such as, the emphasis on the role of language in thought, and, even, a rejection of western rationalism.

These differences are further illustrated by scrutinising Lyotard's postmodernism in relation to the two identified problems of morally uncertain theories. The first of these sets postmodern theory a significant challenge. If it involves incredulity toward metanarratives, then the application of postmodernism generally threatens, itself, to become a metanarrative. The understanding, criticism and approach implied by the postmodern condition could itself qualify as a 'supposedly transcendent and universal truth', which is followed without valid legitimation. This would lead to the application of the postmodern approach to postmodernism itself, or, in other words, to the questioning the very approach of questioning. Some postmodernists might welcome this, coming from such a radically critical perspective, but it does raise the issue of whether there is any truth or validity in adopting this perspective in the first place. If this argument is carried through, postmodernism, ultimately, swallows its own tail, which some may argue is the whole point. As Richard Tarnas suggests: truth and knowledge must itself be regarded as reflecting but one more local and temporal perspective having no necessarily universal, extrahistorical value.... Implicitly, the one 
postmodern absolute is critical consciousness, which, by deconstructing all, seems compelled by its own logic to do so to itself as well. This is the unstable paradox that permeates the postmodern mind. ${ }^{14}$

This first question, then, raises a valid criticism of postmodernism, and one that seems to be answerable only by denying the validity of the position itself. This result is mirrored in relation to the second question. Although there is no substantive moral position that is shared even weakly by postmodernists, what is relatively common is the general postmodern response: one of pluralism, ambiguity and sensitivity to difference. This kind of approach seems to be promoted as appropriate.

Postmodernism, however, cannot easily explain why this is the case. Someone who recognises the incredulity of metanarratives need not necessarily adopt this postmodern approach. That person might decide, instead, to adopt the traditions and truths of western society to the exclusion of others for any number of reasons, or even for no reason at all. The recognition of the shortcomings of western truths does not dictate the adoption of a radical postmodern critique and a pluralist, ambiguous viewpoint; this being the case, postmodernism has no obvious mechanism by which to validate such a response.

The positions of Protagoras and Lyotard contribute towards an understanding of moral uncertainty. This uncertainty encompasses a broad and potentially diverse group of ideas that have in common the scepticism of moral judgments or theories that claim to be objective and universal. These three views, together, allow the basic structure of moral uncertainty to be appreciated and, also, the most basic questions of its viability to be discerned. This uncertainty refers to a group of approaches or theories that are themselves complex, as are the relationships between them. They admit claims that can vary in scope, focus and effect, yet the common thread between them is a belief in the shortcomings of those alternative positions that assert an absoluteness or universality to morality, and a scepticism as to their confidence in being able to apply some valid process to arrive at the morally correct result. They represent moral understanding, instead, in a number of ways: as being in some way contestable, meaning that there is no rational or objective standard by which to judge moral value; as involving deep complexity, not adequately represented in moral theory; and, as being contingent on the specific situations, beliefs and traditions.

\footnotetext{
${ }^{14}$ Tarnas, op cit, above note $4, \mathrm{p} 402$.
} 


\section{Liberal theory}

The beginnings of liberalism as a political ideology are nebulous, partly because the attitude of mind that it represents precedes the use of 'liberal' as a political label. ${ }^{15}$ The promotion of toleration that followed the Wars of Religion is often identified as an important precursor to its development. ${ }^{16}$ The enlightenment also represents a significant period of growth, as public discussion about political liberties became prominent, and the liberal movement later drew inspiration and impetus from the revolutions in both America and France. ${ }^{17}$

The liberal ideology evolved with a number of complementary focuses, the most important being a universal set of individual rights. These included the freedom of conscience and religion, and also economic freedom, as well as a system of private property. ${ }^{18}$ Also important was a constitutional government with a clear separation of powers. ${ }^{19}$ Together, these ideas champion the ability of the individual to be liberated from existing political and cultural power structures. "As a political movement everywhere liberalism emphasized the rights and the potential of the individual facing the forces of tradition and of the old Establishment." 20

The political ideology of liberalism did not evolve in the absence of theorising about its foundations and content. The first liberal work can tentatively be identified as John Locke's Two Treatises on Government, probably written around 1680. Liberal philosophy developed throughout the eighteenth century with contributions from Jean Jacques Rousseau, Thomas Paine and Adam Smith. The nineteenth century saw utilitarianism grow to become a significant political and potentially liberal paradigm, as expressed in the works of Bentham and, probably to a lesser extent, John Stuart

\footnotetext{
${ }^{15}$ E K Bramsted \& K J Melhuish (eds.), Western Liberalism: A History of Documents from Locke to Croce (1978), p3.

${ }^{16}$ Honderich, op cit, above note $1, \mathrm{p} 483$.

${ }^{17}$ Porter, R, Enlightenment (2000), p188; Bramsted \& Melhuish, op cit, above note 15, p3.

${ }^{18}$ Bramsted and Melhuish, op cit, above note 15, pp3-7; J G Merquior, J G, Liberalism, Old and New, (1991), pp15-23.

${ }^{19}$ Bramsted and Melhuish, op cit, above note 15, pp29-31; Merquior, op cit, above note 18, pp23-25.

${ }^{20}$ Bramsted and Melhuish, op cit, above note 15, p3.
} 
Mill. Significant contributions to liberalism were made in the mid twentieth century by Benedetto Croce, Friedrich Hayek and Isaiah Berlin, but by that stage utilitarianism had taken a firm hold over political theory.

The lack of fruitful speculation and development in this area led Berlin to ask, in 1961: “does political theory still exist?" In addressing this question, he identified and described the common belief in the death of political theory:

The principal symptom which seems to support this belief is that no commanding work of political philosophy has appeared in the twentieth century. By a commanding work in the field of general ideas I mean at the very least one that has in a large area converted paradoxes into platitudes or vice versa. This seems to me no more (but also no less) than an adequate criterion of the characteristic in question. ${ }^{21}$

Berlin's conclusion was optimistic: "So long as rational curiosity exists ... political theory will not wholly perish from the earth." ${ }^{22}$ He looked forward for new and unpredictable developments. ${ }^{23}$ He need not have looked far, for, just ten years later, John Rawls published what is probably the most important work of political philosophy of the century. ${ }^{24}$

A Theory of Justice (1971) has the aim of building and justifying a liberal theory of justice on an explicitly non-utilitarian foundation. The theory, itself, is selfconsciously moral, and, because of its aim of being relatively complete and freestanding, is quite complex. It deals with the values of liberty and equality, and the relationship between them. A Theory of Justice has had a profound impact on political philosophy. It stirred a great amount of interest and opinion, and set the agenda for all the liberal theory that was to follow. The discussion and criticism it spawned trace a thread through much of the liberal theory subsequent to its publication. Whether it satisfies Berlin's criterion for a commanding work is arguable. What is clear is that it is the vanguard of contemporary liberal theory, and that any analysis of this area should not fail to take account of its importance as a formulation of a liberal theory of justice, and of its influence on all that has followed.

\footnotetext{
${ }^{21}$ Berlin I, Concepts and Categories: Philosophical Essays (London: Hogarth Press, 1999), p142.

${ }^{22}$ Ibid, p172.

${ }^{23}$ Ibid.

${ }^{24}$ Mautner, The Penguin Dictionary of Philosophy p471.
} 


\section{Method}

\subsection{The perspective of moral uncertainty}

Having broadly discussed the meaning of moral uncertainty, and briefly located contemporary liberal theory within the liberal tradition, it is now necessary to set out how the former will be used to examine the latter. Moral uncertainty has been presented both as a criticism of absolute moral theory, and as an alternative, albeit difficult, approach to thinking about morality. Moral uncertainty presents a fundamental challenge to moral theory. Most theories involve justifying the application of a set of moral judgments generally, whether to one person at some later time, or to all people at all times. The nature of these claims and theories can be quite varied; they feature the same kind of complexity as did the approaches of Protagoras, Russell and Lyotard. As with those theories, moral theories can involve different claims, focuses and arguments, borne out of different methodologies and approaches. There is a complexity of universal positions, just as with positions of uncertainty, that has been explored over thousands of years through concerted intellectual effort, but never reduced to the straight-forward and transparent.

The concern, then, is to analyse how liberal theories respond to moral uncertainty, having accepted the inherent complexity of both. It is not useful to look at liberal theory with a single specific argument or criticism in mind, nor is it possible to consider various forms of liberalism from all possible uncertain positions. Instead, the process will involve examining how a number of alternative liberal theories justify their liberal content, their reasoning from moral foundations to substantive content, in order to assess their attempts, whether explicit or implicit, to respond to moral uncertainty. If some sense, certainty or universality is the aim of a moral theory; viewing it from this perspective means nothing less than assessing its validity and value, albeit from a particular point of view.

Given that moral uncertainty is a prominent criticism of moral theory that relates to its fundamental aims, some measure of recognition and response would be expected. The 
complexity of moral theory allows for a complexity of response, and makes the perspective of uncertainty interesting and useful. The general approach this thesis will take is to examine the aims of a theory, its justification and its substance. In relation to uncertainty, the aims of such a theory will typically be to achieve some generally recognised moral value, or, in other words, some form of universality, objectivity or truth. The justification of a theory represents the strategy by which these aims are to be met, and lays the foundations upon which the substance of the theory is built, by setting out the source of the theory's value. It is this aspect of a theory at which criticisms from moral uncertainty are often aimed, since both have a similar scope, i.e. one asserts an argument for some objective value while the other seeks to undermine that assertion.

Finally, examinations of the substantive parts of each theory are required in order to give a more full and complex picture of each. The moral and political substance of a theory is not as directly relevant to the perspective of uncertainty, yet is essential to the analysis. A theory's substance and principles are the expression of its justification; the two are contiguous, and, as such, an effective examination requires reference to them both. By considering the aims and justificatory strategy of a theory, a general position on it can be reached, but, it is only by considering how these are expressed and developed through the substance (the processes and prescriptions) of the theory, that the full picture is revealed.

It is clear, then, that there are necessary links between each of the aspects of a liberal theory: the aims set the standard for the justification, which gives the reasons for its substantive processes, from which the prescription is derived. Since a theory's justification sets out how the substance of the theory will function in order to achieve its aims, the latter must accord with this explanation in order for the theory to be internally consistent. This means that every aspect of the theory's substantive structure must be within the boundaries set by the justification and, equally, that every essential part of this justification must find expression in the structure. The justification and substance of a theory, then, are not separate, but, rather, the latter builds from and manifests the former in a particular form. 
The method of examining a liberal theory from the perspective of uncertainty, then, should involve identifying and exploring each of its different aspects. By doing this, a detailed view of that theory as a response to uncertainty can be developed. That response can then be brought into question, both, in terms of its internal consistency, and, with respect to its approach to achieving its own aims. In using this method, no single conception of moral uncertainty is required; rather, the analysis is responsive to the structure and substance of the theory itself, yet still challenges that theory from a morally sceptical standpoint.

This method meets the aims of the thesis in that it allows recent liberal theory to be illuminated by a different and significant perspective, and should allow a general analysis of the development of this area of theory. As this process of analysis is applied to various works within the area of contemporary liberal theory, the method itself should develop. Analyses of new theories will add new complexities, and test similar and different approaches to theorising liberalism, leading to an understanding of common challenges and issues such theorising faces.

\subsection{Liberalism examined}

Firstly, the contemporary liberalism that is to be examined will be described uniformly as being theories of liberal justice. This label will be applied for convenience and for the sake of consistence, and follows Rawls' description of his earlier work. This makes no comment on whether particular ideas or systems amount to theories, nor whether justice is their primary concern. 'Theory of justice' could be read, then, as being equivalent to 'proposed system of socio-political organisation'.

The subject matter of the thesis concerns contemporary liberal theories, beginning from Rawls' A Theory of Justice. The primary focus will be on significant examples of liberal theory; in those works that contain a novel and engaging theoretical structure, and that attempts to justify a recognisably liberal conception of justice. Most of the works considered represent mainstream or prominent works in this area. It is important to examine these particular works for a number of reasons: if the 
development of the area is to be charted, then it is necessary to look at those theories that have been most influential on subsequent work; if it is to be examined as a whole, then the central works must be taken account of; and, not least of all, the most successful and well-recognised works are the most likely to be well structured and reasoned, and, so, provide the best place to start looking for well-developed and thought-provoking responses to uncertainty. Because of the inherent limitations of the approach, however, some prominent liberal theories have been excluded. This occurs on the basis that their conceptions of liberalism are either not fruitful, or are substantially reproduced in other examined works.

The works of post-Rawlsian liberalism are typically written by Anglo-American philosophers. Certainly there is a strong non-English tradition throughout the history of liberal theory, but this is not well-represented by the thread of liberalism that is being examined. Some of the writers, either by self-conception or in the view of others, are not liberal, yet, they remain within this post-Rawlsian tradition, and their work contributes to the overall understanding of recent liberal theory.

Problems associated with classifying and grouping different theories, and of locating particular theories within a tradition, are to be expected in such an undertaking. In addressing these challenges, use has been made of both chronology and theme as organising ideas. The former is useful in relation to charting the development of recent liberal thought, and the latter helps in the categorisation of the later chapters, where this liberalism has developed beyond its earlier preoccupations. Inevitably, such organisation cannot do justice to every theory, and must squeeze and truncate some in the name of the overall analysis. The important point is that these works are not so manhandled as to lose their original shape, and, that the overall scheme of an examination of contemporary liberal theory, from the perspective of moral uncertainty, is served.

The idea is to map out the development of Rawlsian and post-Rawlsian liberal theory in relation to its response to liberal uncertainty; its aims at universal justification, and its successes and failures at achieving these. The ultimate aim of this process is to be able to characterise contemporary liberalism on these terms, and to identify the 
directions in which liberal theory can develop, effective both as a moral theory and as a response to moral uncertainty.

\subsection{Summary of thesis}

Chapter 2 will apply the methodology of A Theory of Justice itself. The significance of this work dictates a detailed analysis: from Rawls' justificatory strategy, through his complex theoretical structure and process, to the liberal and egalitarian results. The following two chapters represent early reaction to and development of Rawls' work. Robert Nozick's, Anarchy, State, and Utopia, is often seen as the libertarian response to A Theory. Chapter 3 will examine Nozick's approach to justifying his theory and the similarities and differences between the two, with the focus on this, rather than their respective political consequences.

Ronald Dworkin and Bruce Ackerman both represent recognisably Rawlsian approaches to issues of liberal justice. Their approaches will be detailed in chapter 4 , which will allow for examinations of neutrality and equality as conceptions of liberal theory. Chapter 5 will discuss the communitarian criticisms of liberalism, and looks at a major response to these criticisms, namely the use of pluralism as a foundation for liberalism. This will involve some early post-communitarian liberal conceptions, and the then the later and more thoroughgoing liberal pluralism.

Chapter 6 examines Rawls' shift from the idea of justice as fairness, as expressed in $A$ Theory of Justice, to the separation between the political and the metaphysical, as expressed in Political Liberalism. This approach to liberalism contains a conception of public liberalism, which forms part of its own thread of liberal theory, and this will also be examined in this chapter. Chapter 7 will look at how demands for the recognition of minority cultures have affected liberal theory, and in particular, considers the possibility of culturally-sensitive forms of liberalism. The penultimate chapter concerns Richard Rorty's attempts to build a clearly postmodern version of liberalism. This approach is unique among those surveyed, in that it incorporates a 
radical form of moral uncertainty, and will prove interesting as a final substantive looks at liberalism as a response to uncertainty. 


\section{A THEORY OF JUSTICE}

\section{Introduction}

Rawls' A Theory of Justice (1971) is the vanguard of recent liberal theory, and as such is a useful starting place for a thorough analysis of the subject. It is difficult to understate its significance both as a political theory and as an academic text. It not only signalled the decline of utilitarianism as the dominant paradigm of political philosophy, but also spawned an entire field of political and moral theory. In addition to its scholarly virtues, $A$ Theory drew significant attention and acclaim from the nonacademic press. $^{1}$

There have been a number of reasons given for the success of A Theory of Justice, which include: the way in which it reconciles liberty and equality ${ }^{2}$; the way in which it reconciles fairness and mutual advantage ${ }^{3}$; and, its use of a deontological ethic in relation to justice. ${ }^{4}$ All of these ideas offer some insight into its popularity: it is sufficiently complex and multi-faceted, and the response to it correspondingly so, to support these various interpretations. Inevitably any understanding of it will depend on the context of the reader: their reasons for reading it, and the aspect of it they wish to applaud or criticise.

Rawls himself describes his aim as being to "present a conception of justice which generalizes and carries to a higher level of abstraction the familiar theory of the social contract as found, say, in Locke, Rousseau and Kant." ${ }^{, 5}$ The contract he uses is one that results from the, now well-known, original position. This involves a group of hypothetical people determining what is to be just and unjust in their society. ${ }^{6}$ The outcome of this discussion is Rawls' two principles of justice, liberty and distribution, the former being morally prior to the latter. It is the fairness of the conditions and workings of the original position that lends these principles their legitimacy as

\footnotetext{
${ }^{1}$ N Daniels (ed.), Reading Rawls (1989), pxxxi.

${ }^{2}$ K-C Tan, Toleration, Diversity, and Global Justice (2000), p159.

${ }^{3}$ R Hardin, Liberalism, Constitutionalism, and Democracy (1999), p33.

${ }^{4}$ M Sandel, Liberalism and the Limits of Justice ( $2^{\text {nd }}$ ed., 1998), p15.

${ }^{5}$ J Rawls, A Theory of Justice (Rev ed., 1999), p11.

${ }^{6}$ Ibid.
} 
expressions of justice, and it is for this reason that he describes the main mechanism of the theory as justice as fairness. ${ }^{7}$

These principles entail a position of egalitarianism that reflects many of the actual moral concerns of the era in which A Theory was published. It is true to say of Rawls, then, that:

\begin{abstract}
He wants to reveal the principles of justice which underlie the dominant moral and political views of our period. He wants to show that these principles can be viewed as the result of a selection procedure that all people can agree is fair.... And he wants to show that these principles describe a workable social arrangement, given everything we know from the social sciences. ${ }^{8}$
\end{abstract}

Rawls first presented the idea of an agreement from the original position in 1958, and developed it substantially before publishing it in A Theory in $1971 .{ }^{9}$ Because of this, it is well developed, closely argued, and relatively complete as a political theory. It has the virtues of being both broad in scope and complex in detail. Much of the reasoning is multi-stranded, meaning that there are often several complementary or alternative reasons or explanations for a single argument. Brian Barry, for example, describes Rawls' writings as containing "not one theory of justice, but several, of different levels of abstraction and different degrees of articulation." ${ }^{, 10}$ This detail and complexity means that it can be difficult, in discussing the work, to ensure that its meaning has been usefully captured, without becoming mired in argument and detail. For the purpose of this analysis $A$ Theory should be viewed in a manner conducive to the perspective of moral uncertainty, without compromising the clarity of the view of the work.

It is significant that Rawls himself continued to believe in the correctness of the theory. ${ }^{11} \mathrm{He}$ did, in fact, explain and develop it further following its publication, and defend it against criticism, culminating in a restatement of the central doctrines in $2001 .^{12}$ Given that this analysis is interested in the development of approaches to liberal thought, and in the main assertions of liberal moral value, the primary focus of

\footnotetext{
${ }^{7}$ Ibid.

${ }^{8}$ Daniels, op cit, above note 1 , pxxxiv.

${ }^{9}$ S Freeman, (ed.), John Rawls: Collected Papers (1999), p47.

${ }^{10}$ B M Barry, Essays in Political Theory (1991), p147.

${ }^{11}$ Rawls, op cit, above note 5, pxi.

${ }^{12}$ J Rawls \& E Kelly, Justice as Fairness : A Restatement (2001).
} 
this chapter will be on Rawls' theory as stated in A Theory of Justice. Some of the later developments in his thought will be considered in subsequent chapters, and in particular, the chapter concerning political liberalism.

Rawls' work has spawned a voluminous literature rarely matched by other subjects of academic criticism. There is, as would be imagined, a great deal of overlap between various criticisms, and much of the later material is in relation to more recent developments in liberal theory by Rawls and his critics. The focus here is on those arguments relevant to the view of $A$ Theory as a response to uncertainty, and as a point on the timeline of liberal theory. Many of these arguments will be relevant, particularly those that criticise the justification and moral position implicit in the work, as will some of the more basic criticisms of the theory's approach and substance.

The main task in this chapter, then, is to explore and analyse A Theory of Justice in a way that is conducive to the perspective of uncertainty. This involves examining its method of justification and its substance processes and principles. A successful and morally valuable theory will apply a justificatory strategy that meets its aims and, then, is expressed cogently and consistently by its substance. However for Rawls', as well as many other theories, the main focus is on the substance rather than its justification. Often their aims and justificatory strategies will need to be drawn from some oblique and brief comments, and implied from the other aspects of the theory. For this reason, the approach of working from aim and justification to the substance is problematic. This is exacerbated by the close link identified between these aspects: dealing with each separately and in turn makes it difficult to cross-reference to and connect with matters not yet discussed.

The approach that will be taken in this and other chapters is to initially explore the theory's basic structure - its justification, process and prescriptive principles - as it was originally presented. Here the theory and its reasoning will be taken on its own terms, without undue analysis or criticism. Subsequently, various criticisms of the theory, relevant the perspective of uncertainty, can be canvassed and explored, and the validity and power of the theory tested. This method will allow the analysis of the 
theory to take place after, and in relation to, a presentation of the whole of its structure.

\section{Basic structure}

\subsection{Approach to justification}

By far the most prominent idea in A Theory of Justice is the original position. This mechanism is often identified as the whole of Rawls' theory, and, while it is true to say that this is at its centre, the original position is incomplete as a strategy of justification; only a broader view can offer a proper explanation of the validity and power of Rawls' notion of justice. This involves looking at the original position as part of a reflective equilibrium.

As a justification of his theory, Rawls argues in favour of the necessary moral value of the original position, and the principles of justice it produces. The original position supposes that "one conception of justice is more reasonable than another, or justifiable with respect to it, if rational persons in the initial situation would choose its principles over those of the other for the role of justice." ${ }^{13}$ It is clear, then, that Rawls is asserting that the outcome of the original position has moral value. The nature of the justification of the theory, then, is to show why this is the case.

The original position can be defined by the conditions that constrain its decisionmaking procedure and the principles it produces. The process of finding a reflective equilibrium involves reaching a set of initial conditions that will produce morally relevant results and, then, agreeing with the results that are actually produced. Reaching this equilibrium involves a process of tweaking conditions and results, as well as our own considered judgments relating to both, so that conditions and results are morally significant and one leads logically to the other.

\footnotetext{
${ }^{13}$ Rawls, op cit, above note 5, p16.
} 
In searching for the most favoured description of this situation we work from both ends. We begin by describing it so that it represents generally shared and preferably weak conditions. We then see if these conditions are strong enough to yield a significant set of principles. If not, we look for further premises equally reasonable. But if so, and these principles match our considered convictions of justice, then so far well and good. But presumably there will be discrepancies. In this case we have a choice. We can either modify the account of the initial situation or we can revise our existing judgments.... By going back and forth, sometimes altering the conditions of the contractual circumstances, at others withdrawing our judgments and conforming them to principle, I assume that eventually we shall find a description of the initial situation that both expresses reasonable conditions and yields principles which match our considered judgments duly pruned and adjusted. This state of affairs I refer to as reflective equilibrium. ${ }^{14}$

It is the reflective equilibrium then - this refining of the procedural setup, the principles it produces, and our own considered judgments - that justifies Rawls' use of the original position. The success of this approach depends on the arguments that the conditions he places on the original position are actually morally reasonable, that the principles of justice can be derived from them, and that they plausibly conform to our considered judgments about justice. Much of $A$ Theory is aimed at showing that Rawls' theory does amount to such an equilibrium.

The nature of the justification of $A$ Theory, then, is one of agreement. This agreement appears to occur as part of the operation of the original position, but the truly justificatory agreement is actually external to it. This agreement is with Rawls' assertion that his conception of the original position and the principles of justice amount to a reflective equilibrium: an appropriate moral process with which, upon reasonable reflection, we would all agree. The legitimacy of the theory, then, comes from the claim that every person would recognise it as a reflective equilibrium.

\subsection{Role of the original position}

Although the bulk of $A$ Theory of Justice, and of the responses to it, is dedicated to characterising and exploring the original position, its justificatory structure maps the interplay between the conceptions of the original position and the reflective equilibrium. To demonstrate this structure it is useful to consider the role of each of these. The original position is undoubtedly central to Rawls' theory, yet it would not be fatal for it to be removed entirely. What would remain is the process of comparing

\footnotetext{
${ }^{14}$ Ibid, p18.
} 
the two suggested principles of justice with our considered judgments. Ronald Dworkin has grappled with the idea that the original position could be removed from the theory:

If it has any role, it must be in the process of justification, because it takes its place in the body of theory we construct to bring our convictions into balance. But if the two principles of justice are themselves in reflective equilibrium with our convictions, it is unclear why we need the original position to supplement the two principles on the theoretical side of the balance. What can the idea contribute to a harmony already established ? $^{15}$

The provisional answer he gives is that it is "one of the conditions we impose on a theoretical principle, before we allow it to figure as a justification of our convictions." ${ }^{\prime 16}$ And this is essentially the role that the original position takes in the justificatory process. By requiring that the principles of justice conform to the original position, these are subjected to additional moral criteria, as manifested in the conditions and the workings of the position. This allows a more complex interplay between the conditions, principles and our considered judgments, and so contributes to the moral content of the theory.

It is the actual working of the original position, through the mechanism of individual choice in a constrained situation, that produces the principles of justice. It is this mechanism that links the moral conditions to principles in an acceptable way. As Thomas Nagel has put it,

[t]he aim is to provide a way of treating the basic problems of social choice, for which no generally recognized methods of precise solution exist, through the proxy of a specially constructed parallel problem of individual choice, which can be solved by the more reliable intuitions and decision procedures of rational choice. ${ }^{17}$

The role of the original position, then, is to buttress the reflective equilibrium by adding several conditions and a closely defined procedure, all of which are recognisable, either morally or as rational choice. Because of this, much of $A$ Theory of Justice is aimed at demonstrating the value of both of the conditions and the procedure of the original position. Without the original position, the process of working towards a reflective equilibrium would be deficient, and would suffer a corresponding fall in prescriptive moral power. In justificatory terms, then, the

\footnotetext{
${ }^{15}$ Dworkin, R M, Taking Rights Seriously (London: Duckworth, 1978) p156.

${ }^{16}$ Ibid, p157.

${ }^{17}$ T Nagel, 'Rawls on Justice', in Daniels, op cit, above note 1, p1.
} 
original position is embedded in the content of the process of reflection. This process is itself the means by which the theory can assert moral value, and so has a necessary role as part of the justificatory structure. It is this process by which a theory can be found to be a reasonably acceptable conception of justice, and which Rawls is trying to persuade us is satisfied by the content of his theory.

\subsection{Conditions of the original position}

The justification of $A$ Theory of Justice, then, involves a reasonable agreement with Rawls' assessment of the original position and its principles as a valid expression of justice. Having identified this, the next move is to examine its expression in the substance of the theory. Here, the two substantive aspects of process and prescription are readily met by the procedure of the original position and the two principles of justice respectively. ${ }^{18}$

For the justification to be effective, there are two related requirements of the original position: its conditions must be morally relevant, and the procedure by which it functions must be fair. Rawls explicitly makes both of these arguments. ${ }^{19}$ The original position is a hypothetical contract formed between representative parties. ${ }^{20}$ This hypothetical situation is constrained in a number of ways, the most significant of which is the veil of ignorance. This means that the individual parties to the contract are unaware of their place in society, class position and social status, and their conception of the good, as well as their "fortune in the distribution of natural assets and abilities, [their] intelligence, strength and the like". ${ }^{21}$ In addition, they know not the circumstances of their society, economic or political details, or information about their civilization or culture. ${ }^{22}$ What they do know includes facts about human nature, and political and economic affairs generally. ${ }^{23}$ The idea is that matters that are not relevant to decisions about justice, such as those which would inform people's self-

\footnotetext{
${ }^{18}$ The principle of liberty and the difference principle.

${ }^{19}$ Rawls, op cit, above note 5, pp19, 105 and pp15, 104 respectively.

${ }^{20}$ Ibid, pp11, 82 .

${ }^{21}$ Ibid, p118.

${ }^{22}$ Ibid.

${ }^{23}$ Ibid, p119.
} 
interest or prejudice, are excluded from the decision-making process, while the individuals are acquainted with other, more relevant general information. Rawls argues that the exclusion of this personal information gives the deliberation of each person more moral significance; it means that people will not be inclined to reach political principles that privilege themselves, their class or conception of the good, and allows a widespread agreement between people on matters of justice. It is meant to exclude morally arbitrary factors and, so, force the parties to seek just and fair principles. It is this condition of the original position that carries the most justificatory weight, in that it has some intuitive moral force and attaches power to the result of the process.

A second condition of the original position sets out the equality of all parties to the agreement. This means that each has an equal role, and equal rights, in engaging in the contracting process. ${ }^{24}$ However, the full sense in which the parties to that agreement are equal is more complex and subtle. The veil of ignorance is itself an equalising mechanism: it takes a diverse group of individuals with different backgrounds, beliefs and conceptions of the good and effectively removes these differences. The result is that each person applies to the agreement process their sense of justice, which is shared by all, since all are morally capable people. ${ }^{25}$ In fact, Dworkin identifies the implicit fundamental concept of the original position to be an equal concern and respect for all individuals. ${ }^{26}$ Thus, equal consideration should be recognised as a condition of the original position and must be acknowledged in the process of finding a reflective equilibrium.

The final condition of the original position is that of the rationality of the representative parties. In explaining how they are rational, Rawls has recourse to a concept of 'primary goods'. These are goods that, it is presumed, every rational person would want. They include social goods, such as rights, liberties, opportunities, income and wealth, as well as natural goods, such as health, intelligence and imagination. ${ }^{27}$ Individuals in the original position, despite the limits on their

\footnotetext{
${ }^{24}$ Ibid, p17.

${ }^{25}$ Ibid.

${ }^{26}$ Dworkin, op cit, above note 15 , p181.

${ }^{27}$ Rawls, op cit, above note 5, p54.
} 
knowledge, are able to make rational decisions. The obvious such decision is to prefer more of a primary good to less.

The use of a conception of rationality is necessary in order to predict how the parties to the original position would act and the content of the agreement they would reach, and to argue that this would indeed be common between all the parties. As a condition of the original position, however, this rationality must be shown to be morally acceptable, and must fit with the veil of ignorance in the sense that each party must act rationally given the nature of the information that they are permitted. Also, the rationality must satisfy the assertion that the contracting process will lead to a fair result. One particular concern might be that people in the original position will act in a self-interested manner and, so, undermine the resulting principles. In fact, this rationality does imply that each person acts to further their own purposes. ${ }^{28}$ But, the restrictions implied by the veil of ignorance suggest that individuals are incapable of being ruthlessly egoistic, for they are not aware of which principles would particularly favour them. ${ }^{29}$ This means that they are forced, instead, to view the good of society as some complete whole and to consider the positions of each possible individual, for they cannot be sure which of these they will turn out to be. This rather elegant result that the moral weakness of rationality is cancelled out by restrictions on knowledge shows the mechanism by which individual choice is used as a proxy for social choice, and sets the foundations for the derivation of actual principles from the original position. $^{30}$

\subsection{Outcome of the original position}

The principles of justice that Rawls uses must be the logical outcome of the original position. In other words, "given the circumstances of the parties, and their knowledge, beliefs, and interests, an agreement on these principles is the best way for each person to secure his ends in view of the alternatives available". ${ }^{31}$ Rawls argues that his two

\footnotetext{
${ }^{28}$ Ibid, p124.

${ }^{29}$ Ibid, p129.

${ }^{30}$ Nagel, op cit, above note 17.

${ }^{31}$ Rawls, op cit, above note 5, pp102-103.
} 
principles would be chosen over a number of possible alternative political principles, including utilitarian and perfectionist principles. ${ }^{32}$ This does not indicate that they are correct or true principles, but, rather, that they are the best expressions of justice among the list of reasonable alternatives, and, so, are a potential part of a reflective equilibrium. Thus, the process of comparing principles from the perspective of the original position "moves us closer to the philosophical ideal; it does not, of course, achieve it.",33

The approach of deriving Rawls' principles from the original position involves two steps, setting out the reasoning that leads to each principle in turn. Rawls argues that the principle of liberty is the first assertion any rational person in the original position would make, and reflects the equality of individuals once personal concerns are removed.

\begin{abstract}
Since it is not reasonable for him to expect more than an equal share in the division of social primary goods, and since it is not rational for him to agree to less, the sensible thing is to acknowledge as the first step a principle of justice requiring an equal distribution. Indeed, this principle is so obvious given the symmetry of the parties that it would occur to everyone immediately. Thus the parties start with a principle requiring equal basic liberties for all, as well as fair equality of opportunity and equal division of income and wealth. ${ }^{34}$
\end{abstract}

This principle of liberal equality, then, is argued to be the basic and obvious response to the conditions of the original position and, as such, necessarily embodies the virtue of fairness, and, so, is just. This principle entails that "each person is to have an equal right to the most extensive scheme of equal basic liberties compatible with a similar scheme of liberties for others." ${ }^{35}$ Rawls offers no precise or comprehensive list of such rights, but does explore the kinds of rights that might be covered, including looking at the equal liberty of conscience and of the person. ${ }^{36}$

The second step in arguing from the original position begins where the first left off. The principle of equal social primary goods is the most sensible initial move, but it is inherently static. It sets out a position of distribution and right that can be met at a

\footnotetext{
${ }^{32}$ Ibid, p43.

${ }^{33}$ Ibid.

${ }^{34}$ Ibid, p130.

${ }^{35}$ Ibid, p53.

${ }^{36}$ Ibid, p180.
} 
particular point in time, but does not set out how later actual agreements or moves that take the position away from this equality are to be treated. Rawls reasons that

[s]ociety should take into account economic efficiencies and the requirements of organization and technology. If there are inequalities in income and wealth, and differences in authority and degrees of responsibility, that work to make everyone better off in comparison to the benchmark of equality, why not permit them? ... Thus the basic structure should allow these inequalities so long as these improve everyone's situation, including that of the least advantaged, provided they are consistent with equal liberty and fair opportunity. ${ }^{37}$

The result Rawls calls the difference principle. This is an outcome of the original position subsequent to the first principle of liberal equality, since " $[\mathrm{t}]$ aking equality as the basis of comparison, those who have gained more must do so on terms that are justifiable to those who have gained the least." 38 The reasoning behind the difference principle emphasises that it does not depend on any person knowing how an inequality will affect his or her own interests; the contractors agree to the principle because, irrespective of how the inequality affects them, it can make them no worse off relative to the baseline of equality. This allows that the obvious and significant gains from, say, allocating a task, position or role to a person to whom it is suited by reason of talent or experience, can be realised. Everyone can agree to this from behind the veil of ignorance since no-one will be made worse off relative to the position of equality, and all will have a fair opportunity to attain this special status, or to benefit from this inequality. ${ }^{39}$

However, the effect of the difference principle also extends to voluntary market transactions. In most cases these will benefit all affected parties, and so are potentially sanctioned by the difference principle. Nevertheless, the action of the market must remain within "a framework of political and legal institutions which regulates the overall trends of economic events and preserves the social conditions necessary for fair equality of opportunity." 40

These two principles represent Rawls' predicted outcome of the original position. The one point that remains concerns the relationship between the two. The fact that the first principle is a consequence of the initial response of equality, and that this sets the

\footnotetext{
${ }^{37}$ Ibid, pp130-131.

${ }^{38}$ Ibid, p131.

${ }^{39}$ Ibid, p63.

${ }^{40}$ Ibid.
} 
baseline from which the difference principle is drawn, suggests that the latter is logically subordinate to the former. And this is exactly the position that Rawls takes: that the liberty principle is lexically (lexicographically) prior to the difference principle. ${ }^{41}$ This term is used because the way in which the principles are ordered resembles the way words are listed alphabetically: only when the first letters are the same does the second letter become relevant. This places the two principles on entirely separate levels, and means that matters affecting liberty should always be considered prior to those concerning inequality or, in other words, that only liberty can limit liberty. ${ }^{42}$

Rawls also argues that these two principles and the relationship between them is completely reasonable in a politically pragmatic sense: they tend to promote the good of individuals, tend to be stable, and will promote self-respect and social co-operation and a mutual public respect. ${ }^{43}$ They mean that all people will be treated as ends in themselves, and that their interests will not be traded off for the good of others or of the majority. ${ }^{44}$ These assertions satisfy our beliefs about justice and politics, in addition to being the result of a procedure that must produce fair principles. The intuitive value of each of these aspects of Rawls' theory builds a strong justificatory argument that these principles represent a valid reflective equilibrium of justice, and that they ought to be adopted by any state and society.

\section{Analysis}

\subsection{Universal aims}

The first task of analysing Rawls' theory is to identify the basic position in relation to uncertainty, as represented by its universal aspirations. It is clear that the nature of the theory and its approach to justification does not involve the assertion of an absolute, perfect and unchangeable truth. Amy Gutmann, for example, has commented that

\footnotetext{
${ }^{41}$ Ibid, p38.

${ }^{42}$ Ibid, p214.

${ }^{43}$ Ibid, pp154-156

${ }^{44}$ Ibid, p156.
} 
[g]rand theorists as different as Plato, Hobbes and Mill wrote as if their conceptions of justice were justified for all people at all times. They searched for a set of eternal forms, a self-evident truth, or a very simple first principle from which to derive all practical moral imperatives. In defending his method of "rational equilibrium," Rawls is the most modest, and in this respect wisest, of the grand philosophers. He argues that we have no better way of justifying principles... than by translating [them] into social practices and judging whether these practices are consistent with our moral convictions. ${ }^{45}$

Thus, Rawls' reliance on a process of testing principles of justice against out own moral judgments limits the potential value of the theory relative to those asserting an absolute truth. Rawls himself states that

I do not claim for the principles of justice proposed that they are necessary truths or derivable from such truths. A conception of justice cannot be deduced from self-evident premises or conditions on principles; instead its justification is a matter of the mutual support of many considerations, of everything fitting together into one coherent view. ${ }^{46}$

Rawls here rejects the value of absolute moral theory. The position of a reflective equilibrium involves the 'fitting together' of our considered judgments, some proposed principles of judgments, and our acceptance of both the conditions and workings of the original position. It is the potential coherence of these ideas, Rawls argues, that can serve to justify a moral theory, rather than by reference to some universal rule or truth. This realisation does not mean that his theory is relative, or eschews the claim of universality. In fact, as Rawls makes clear throughout, A Theory is aimed at justifying the two principles of justice from a perspective common to all people, and so awarding them some universal value. This is readily apparent from the final passage of the work:

The perspective of eternity is not a perspective from a certain place beyond the world, nor the point of view of a transcendent being; rather it is a certain form of thought and feeling that can, whatever their generation, bring together into one scheme all individual perspectives and arrive at regulative principles that can be affirmed by everyone as he lives by them, each from his own standpoint. ${ }^{47}$

Rawls seems to be asserting that a universal moral standpoint is possible, even though it cannot take the form envisaged by grand or absolute theory. This typifies his overall approach while justifying $A$ Theory: he aspires to achieve a universal set of liberal principles, but accepts the inherent limitations of moral theory and thought. ${ }^{48}$ This acceptance is manifest throughout $A$ Theory: in the way Rawls compares his two

\footnotetext{
${ }^{45}$ A Gutmann, 'The Central Role of Rawls' Theory' (1989) 36(3) Dissent 338, p342.

${ }^{46}$ Rawls, op cit, above note 5, p19.

${ }^{47}$ Ibid, p514.

${ }^{48}$ See, for example, p507.
} 
principles against other likely approaches, rather than presenting it simply as the correct result derived directly from true premises; in the way he presents his result as being part of a potentially larger process, and not an incontestable endpoint; and in the means of justification itself, by relying on the considered judgments of the reader. However, while the approach is necessarily imperfect and the results only an approximation of justice, they do refer to a true justice, and represent a more accurate transcript than any known alternative approach. The 'perspective of eternity', although not perfect, is common between all people in all contexts, and it is inherent in Rawls' approach that it can be employed to discern the best of a number of moral theories.

This expresses a basic response to uncertainty: an acceptance of the limitations of the pursuit of moral knowledge, coupled with the belief that, by the application of both reason and our considered judgments, we have the ability to recognise the shape and content of justice. In this way, Rawls accepts and incorporates many of those arguments that are sceptical of the power of moral theory, yet attempts to overcome their basic consequence, that no objective or universal results are possible. It is this approach that prompts Gutmann to class Rawls among the grand philosophers. The success of $A$ Theory as an expression of justice, then, depends on Rawls achieving this universal moral position while acknowledging the limitations of moral theory. Determining whether this is achieved involves analysing the theory's justificatory strategy and, in particular, how these limitations on moral theory are conceived, and how they are overcome by his approach.

\subsection{Justification and the original position}

Rawls' approach to justification involves the formulation of the original position and the resulting principles of justice as a reflective equilibrium. Analysing this approach will involve looking at two common arguments made against it, relating firstly to the contractual aspect of the original position and then the legitimacy of using our considered judgments in the process of looking for a reflective equilibrium. This will 
be followed by an examination of the features of the original position itself, and finally, the principles of justice.

As a matter of justification, it is essential that the conditions of the original position are moral, and it necessarily produces a fair result. The focus here is on the nature and value of the agreement that the original position represents. As was mentioned above, Rawls views one of his main aims as presenting a theory that "generalizes and carries to a higher level of abstraction" traditional contractarian approaches to political theory. ${ }^{49}$ In very basic terms, these approaches involve identifying some social contract with which people agree, whether explicitly or implicitly. This contract then legitimises the actions of the government that are within its ambit. The idea of deriving legitimacy from agreement or consent is an old one in political and social philosophy.

Rawls' contract differs from more traditional approaches in that the situation it represents is hypothetical. This has led to significant difficulty in identifying its meaning and justificatory role. Michael Sandel, for example, identifies the contract in the original position as being doubly hypothetical in that "[i]t imagines an event that never really happened, involving the sorts of beings who never really existed." ${ }^{, 50} \mathrm{He}$ argues that even actual contracts are not "self-sufficient moral instruments", and can only be understood within a broader moral context: one that would encompass the positions of the parties, the details of bargaining process, and the nature of the actual obligations that arise. ${ }^{51}$ The contracting process, then, does not by itself guarantee a fair result, even when there is actual agreement between actual parties.

Dworkin also questions the role of the contract within A Theory. He examines its hypothetical nature and decides that such an agreement is not legitimised merely by virtue of being a contract. The role of the contract, then, must be that it "call[s] attention to some independent argument for the fairness of the two principles - an argument that does not rest on the false premise that a hypothetical contract has some

\footnotetext{
${ }^{49}$ Ibid, p10.

${ }^{50}$ M J Sandel, Liberalism and the Limits of Justice (2 ${ }^{\text {nd }}$ ed., 1998), p105.

${ }^{51}$ Ibid, p109.
} 
pale binding force". ${ }^{52}$ Consequently, if the contract is to have an effect it must be as part of some other argument, and not merely because of its nature as a contract. Together, Sandel and Dworkin can be taken as arguing that the two principles of justice are not legitimised because they are the subject of a contract, and that any such legitimising process is dependant on the contract's context, both in terms of the details of the bargaining situation, and the broader justificatory strategy within which the contract is embedded. But, this does not mean that the value of any such contract is negated; rather, that it is not sufficient justification by itself of any obligation or principle.

Valid though this argument may be, it is not clearly in conflict with A Theory. The original position is constrained in a way that is supposed necessarily to produce a fair result. Its legitimacy, then, is dependant on our recognition and acceptance of its details, and not the status of the agreement reached. In this sense, the value of the contract comes not from it being a contract, but rather from our own moral judgment of the original position as a whole. This means that the 'contract' can be a part of the theory's justification without falling afoul of Dworkin's and Sandel's concerns.

Despite this, the role of such a contract as part of a justification is difficult and potentially misleading, since it seems to add nothing to the moral value of the theory. Unlike traditional contractarian arguments, no justificatory weight is placed on either the consent or agreement aspects of the contract. Sandel recognises that the agreement in the original position is not the kind of agreement that is reached as the result of discussion within a diverse group of people, but, rather, is in the nature of an individual agreeing to a proposition. Such an agreement

amounts to acknowledging [the proposition's] validity, and this requires neither that others be involved nor that I take the validity of the proposition to be a matter of choice. It may be enough that I see it to be valid, as when I agree to (or accept, or acknowledge) the proposition that $2+2$ $=4 .^{53}$

Strictly speaking, then, the original position would carry the same weight even if it involved the decision of just one representative person. ${ }^{54}$ Thus, it is an exercise in morally constrained choice, and not deliberation or agreement. Consequently,

\footnotetext{
${ }^{52}$ Dworkin, op cit, above note $15, \mathrm{p} 152$.

${ }^{53}$ Sandel, op cit, above note 48, p130.

${ }^{54}$ See also R M Hare, 'Rawls' Theory of Justice II' (1973) Philosophical Quarterly 241, p244.
} 
although the point made by Sandel and Dworkin does not undermine Rawls' justificatory approach, it does remind us that, in applying our considered judgment to the original position, the normal contractarian intuitions relating to consent and agreement are not relevant. The only role of the contract in the original position, then, is to make clear the point that every possible representative person who is constrained in that same way would reach the same decision. This may contribute to our judgment of the fairness of the result, but the fact that Rawls presents it in the form of a contract is not relevant to its moral value. This seems to undermine Rawls' claims as to the importance of the contractarian aspect of $A$ Theory, yet it is in accordance with the most reasonable reading of his justificatory approach, and avoids what Sandel and Dworkin have correctly identified as a difficult or even untenable position.

Overall, this means that the agreement in the original position can play a valid part in justifying Rawls' theory, but that this depends on recognising its conditions - the veil of ignorance etc. - and the inherent fairness of the processes involved. These are to be considered, along with the actual result of the process, to determine whether the standard of a reflective equilibrium has been reached. Rawls asserts that his conception of the original position and the two principles of justice represent an equilibrium with which everyone, upon consideration, could agree. The remainder of the analysis of his justification of $A$ Theory will look at the role considered judgment plays in this process, and what this sort of agreement, if achievable, could mean.

\subsection{Moral intuitions and considered judgments}

The process of recognising a reflective equilibrium relies heavily on the use of our considered judgments, both in relation to its conditions and the resulting principles of justice. Rawls defines these judgments in the following way:

Considered judgments are simply those rendered under conditions favourable to the exercise of the sense of justice, and therefore in circumstances where the more common excuses and explanations for making a mistake do not obtain. The person making the judgment is presumed, then, to have the ability, the opportunity, and the desire to reach a correct decision.... Moreover, the criteria that identify these judgments are not arbitrary. ${ }^{55}$

\footnotetext{
${ }^{55}$ Rawls, op cit, above note 5, p42.
} 
These judgments, then, amount to moral intuitions that are properly directed towards matters of justice, and reasonably constrained to exclude potentially distorting influences and considerations. Their role in the justification of Rawls' theory makes clear that he is relying on a form of intuitionism, in the sense that our actual moral judgments are one of the criteria upon which the principles, and in fact the whole of the theory, are being judged. ${ }^{56}$ If the challenge of Rawls' theory is to achieve universality while adopting some of the limitations of moral theory, then intuitions represent the main mechanism by which this is to be achieved. The universality is proved not by the truth of some underlying rule or doctrine, but by the agreement between people who direct their considered judgment at the theory. As Nagel has put it:

[Rawls] intends ... that the underlying principles should possess intuitive moral plausibility of their own, and that the total theory should not merely summarize but illuminate and make plausible the particular judgments that it explains. Moreover, its intrinsic plausibility may persuade us to modify or extend our intuitions, thereby achieving greater theoretical coherence. ${ }^{57}$

The interplay here is between our considered judgments of the principles of justice and of the original position. The aim is to arrive at a coherent position that is characterised by our willingness to accept not just the plausibility of each part of the theory, but the manner in which each part relates to and supports the other, and how this is reflected by, and also informs, our intuitions. Since our own considered judgments will themselves be modified and illuminated in this process, the important intuitive standard is that of plausibility: if we find a position plausible then we have recognised sufficient intuitive value in it that we may be willing to accept it as the most appropriate moral position given its necessary relationship to the other parts of the theory.

The approach of searching for a plausible and coherent equilibrium can be contrasted with more traditional grand moral theory, whereby moral prescriptions are derived from some rule or doctrine that is presented as being absolutely true. It has the virtue of relying not on abstract theory, but rather on our actual moral feelings and beliefs about justice. However, it is difficult to see how some of our intuitions indicate a

\footnotetext{
${ }^{56}$ The use of 'intuitionism' here is different than the sense in which Rawls actually uses it.

${ }^{57}$ Nagel in Daniels, op cit, above note 1, p2.
} 
universal value, and why Rawls' approach should be considered successful in identifying which of our intuitions possess this quality.

This difficulty has prompted a great deal of criticism. One of the most belligerent attacks is from R M Hare, who argues that Rawls' approach hides a bias that undermines the theory. He asserts that Rawls' claim of universality is a matter of selfdeception, which means that "if (as will certainly be the case) [Rawls] finds a large number of readers who can share with him a cosy unanimity in their considered judgments, he and they will think that they adequately represent 'people generally', and congratulate themselves on having attained the truth." ${ }^{, 58}$ In this way Hare argues that Rawls' view from eternity is actually just Rawls' personal view, and that the use of intuitions merely disguises what he is actually doing, which is presenting his own idiosyncratic and morally unjustified beliefs as the correct view of justice. Hare goes on to illustrate this by identifying the extent to which Rawls' theory relies explicitly on intuition and belief:

\begin{abstract}
I have counted in two pages thirty expressions implying a reliance on intuitions: such expressions as 'I assume that there is a broad measure of agreement that'; 'commonly shared presumptions'; 'acceptable principles'; 'it seems reasonable to suppose'; 'is arrived at in a natural way'; 'match our considered convictions of justice or extend them in an acceptable way'; 'which we can affirm on reflection'; 'we are confident'; 'we think'; and so on. ... Since the theoretical structure is tailored at every point to fit Rawls' intuitions, it is hardly surprising that its normative consequences fit them too - if they did not, he would alter the theory ... and the fact that Rawls is a fairly typical man of his times and society, and will therefore have many adherents, does not make this a good way of doing philosophy. ${ }^{59}$
\end{abstract}

This claim, that Rawls' persistent reliance on intuition undermines the universality of his theory, should seemingly be easy to test. Rawls claims that this universality comes from a "certain form of thought and feeling" of all people on matters of justice, and that leads to intuitions and results that are common between them. ${ }^{60}$ Rawls', in effect, argues that his own conception of a theory of justice is an example of this universality. If he is right then every person, regardless of background and belief, who considers this matter ought to agree with this conception. The existence of persistent and rational disagreement should then signal the non-universality of $A$ Theory.

\footnotetext{
${ }^{58}$ R M Hare, 'Rawls' Theory of Justice I' (1973) Philosophical Quarterly 144, p145.

${ }^{59}$ Ibid, pp146-147.

${ }^{60}$ Rawls, op cit, above note 5, p514.
} 
Of course, A Theory has not met with anything approximating the required level of agreement, which seems to undermine its whole project. This is not directly addressed, but it is a consequence that Rawls would no doubt deny. He might argue that the intuitions involved do not reach the standard required by considered judgments, by being the result of a mistake, by taking into account considerations not relevant to the matter of justice, or by including non-arbitrary criteria for example. This would be the case if readers consider the theory with some established moral or political agenda, and not as a pure conception of justice.

Rawls might also add that this disagreement merely reflects the inherent limitations of moral theory: although his strategy of justification implies some theoretical possibility of consensus, in practice this is not achievable because, for example, of the imprecision of language and the finite space available. Or he might argue more generally that such an agreement is simply unrealistic, given the complexity of what is at stake. Each of these arguments has some merit, yet they seem inadequate to explain the lack of agreement with $A$ Theory, particularly when it is this agreement upon which it ultimately depends: it is quite possible to follow all of Rawls' directions and prescriptions and still to disagree with Rawls' conclusions.

Rawls comes closest to dealing with this issue in making some remarks about the nature of a reflective equilibrium:

[D]oes a reflective equilibrium (in the sense of the philosophical ideal) exist? If so, is it unique? Even if it is unique, can it be reached? Perhaps the judgments from which we begin or the course of reflection itself (or both), affect the resting point, if any, that we eventually achieve. It would be useless, however, to speculate about these matters here. They are far beyond our reach. I shall not even ask whether the principles that characterize one person's considered judgments are the same as those that characterize another's... If men's conceptions of justice finally turn out to differ, the ways in which they do is a matter of first importance.... If we can characterize one (educated) person's sense of justice, we might have a good beginning toward a theory of justice ... So for the purposes of this book, the views of the reader and the author are the only ones that count. $^{61}$

Rawls' actual response to this potential disagreement seems to be to downplay the significance and universality of his theory of justice. This is expressed most obviously when Rawls allows that conceptions of justice may differ between individuals, and that his theory might play a useful role if it does nothing more than characterise the

${ }^{61}$ Ibid, pp43-44. 
conception of one person. This suggestion culminates in the direction that the reader should be concerned only with his or her own view of the theory, and should ignore the issue of a widespread consensus. This does not sit easily with the universal elements of Rawls' justification: conceivably, the view from eternity is not only his or his reader's, and does not adequately counter Hare's criticism. Instead it seems only to encourage further examination of the use of considered judgments as a criterion of justice.

The argument against this intuitionism involves two separate issues: firstly, the role of our intuitions in identifying universal moral principles, and secondly, the claim that the two principles of justice exhibit such universality. Rawls' inclusion of our intuitions, as considered judgments, in the justificatory process is itself significant. It certainly seems a sensible and plausible position that our actual beliefs and intuitive responses to situations are relevant to justice. An approach that incorporates our intuitions while attempting to reach universal results faces the general and related issues of, firstly, finding some relationship between our actual intuitions and what our moral principles ought to be, and, secondly, incorporating our intuitions within a logical structure. Rawls' solution to the latter is exceedingly clever: use our intuitions of both the original position and resulting principles as part of a dynamic process of comparison and evaluation, with the aim of reaching a coherent, mutually reinforcing position. The problem is in relation to the former: he never adequately explains the relationship between our intuitions and universal justice, and, so, never plausibly justifies his conception of this position, beyond it being the expression of his personal beliefs. The result is a conception that is plausible, or even somewhat seductive, at least to western sensibilities, but which is accompanied by the nagging feeling of being free-floating, tethered to nothing more than the ideas of one person, or of an aspect of one tradition.

This conclusion does not necessarily deny the universality of Rawls' theory, but it does suggest that his justificatory approach is incapable of supporting this result, in particular because the relationship between our considered judgments and justice is not adequately explained. Consequently, the existence of considered and rational disagreement with the reflective equilibrium, and Hare's argument as to Rawls' parochial moral content, raise issues that go unanswered by $A$ Theory. This offers a 
basic criticism of the justificatory process underlying $A$ Theory. In order to gain a more complete picture of the theory from the view of moral uncertainty, it is necessary to examine the theory's substantive processes and principles as well. These are contiguous with and are an expression of its justification and, despite the general and foundational importance of the latter, a complete view of the theory cannot be achieved without this.

\subsection{Deontology, the right and the good}

The deontological nature of Rawls' theory, and its expression as the priority of the right over the good, is the fundamental organising principle of its substance, and has been described both as contemporary liberalism's great virtue and as its foundational flaw. ${ }^{62}$ Rawls defines deontological theory in the negative sense of being nonteleological, in that it "either does not specify the good independently from the right, or does not interpret the right as maximizing the good". ${ }^{63}$ His theory is deontological in the second sense, since the two principles of justice do not tend to maximise the good, and instead the right is separate and prior to the good. ${ }^{64}$

The nature of deontological moral theory, then, is that the right is not exclusively determined by the good. ${ }^{65}$ For Rawls, this means that the justice of a particular social arrangement can be found primarily in expressions of the right, such as individual liberties, institutional fairness and equality, rather than being derived from the welfare of some individual or group, whether expressed as their preferences, happiness or desire, hence the priority of the right over the good in matters of justice. This does not mean that the consequences of a situation are not morally relevant, but rather that the welfare of those involved plays a role secondary to the rightness of the action itself. By this general deontological approach, and in applying the priority of the right over the good, Rawls structures the substance of much of his theory and steers it away from the utilitarian (and teleological) conclusion that the right is defined by the good.

\footnotetext{
${ }^{62}$ W Kymlicka, 'Rawls on Teleology and Deontology' (1988) 17(3) Philosophy and Public Affairs $173, \mathrm{p} 173$.

${ }^{63}$ Rawls, op cit, above not 5, p26.

${ }^{64}$ Ibid, pp26-27.

${ }^{65}$ T Mautner, The Penguin Dictionary of Philosophy (2000), p131.
} 
He characterises this priority in a number of sections of $A$ Theory, but gives relatively little attention to its justification. In introducing the idea, Rawls sets out that

[i]t has seemed to many philosophers, and it appears to be supported by the convictions of common sense, that we distinguish as a matter of principle between the claims of liberty and right on one hand and the desirability of increasing aggregate social welfare on the other; and that we give a certain priority, if not absolute weight, to the former. Each member of society is thought to have an inviolability founded on justice, or as some say, on natural right, which even the welfare of every one else cannot override.... Therefore in a just society the basic liberties are taken for granted and the rights secured by justice are not subject to political bargaining or to the calculus of social interests. ${ }^{66}$

As a justification of the priority of right, this passage is quite cursory, and cites as supporting evidence 'many philosophers' and 'common sense'. His basic claim is that the concerns of welfare are inferior in relation to justice, to matters of liberty and right. A significant source of this seems to be the intrinsic worth of all people, which means that it would be wrong for one person's liberty to be traded off for the welfare of another.

This basic conception of the priority of the right is quite plausible, despite being inadequately justified in A Theory. The general problems with depending on this sort of intuitive plausibility have already been identified and discussed. The priority of the right over the good, in fact, offers a prominent example of the way that these intuitions can play a role in the substance of the theory, in addition to its justification. A detailed examination of this priority will illustrate some of these issues, and also assist in explaining the substantive structure of $A$ Theory.

It is well recognised that Rawls' use of the priority of the right over the good involves two potentially distinct claims: that some rights are so important that they cannot be traded off with welfare, and that the justification of a theory of justice should not depend on a particular conception of the good. ${ }^{67}$ The first of these has been briefly explained. The latter expresses the idea that the organisation of society should not depend on any one person's or group's idea of how people ought to live, but, instead, should be based on more general and fundamental moral concerns, such as those relating to appropriate individual liberties. This makes it a concept particularly amenable to a liberal framework. This is reflected both in the justification and

\footnotetext{
${ }^{66}$ Rawls, op cit, above note 5, pp24-25.

${ }^{67}$ Sandel, op cit, above note 48, px; Kymlicka, op cit, above note 60, pp185-186.
} 
substance of Rawls' theory: in the former, in that our considered judgments about the various aspects of the theory that contribute towards any reflective equilibrium explicitly exclude any arbitrary considerations, such as any individual conceptions of the good; and, in the latter, primarily because the veil of ignorance precludes anyone in the original position from having prior knowledge of their own conception. ${ }^{68}$ These conditions mean that $A$ Theory is conceived independently of any particular conception of the good. If either were breached this would undermine the universality of the theory since, as Rawls conceives them, the right is moral and common between people, whereas the good is diverse and dependant on each individual's beliefs and backgrounds.

The other use of the priority of the right over the good dictates that a person's liberties or moral rights cannot be traded off for the welfare of any other person or group. This is expressed in the theory in large part by the role of two principles, and, particularly, in the special status of those rights protected by the liberty principle. These take absolute precedence over matters of material welfare; they cannot be abridged or abrogated for the good of another. This use of the priority of the right is linked to the other in the sense that it is the prescriptive expression of the theoretical structure the other implies. Together they construct a conception of justice independent of our personal motives and interests, which promotes a system of universal inalienable rights and satisfies our deeply-felt intuitions concerning the relationship between what is right and what is good. This approach is prominent not only in A Theory, but flows through much of contemporary liberal theory. It claims universality in that it is by design independent of our individual beliefs and ideas of how it is best to live. The resulting structure is so strongly appealing that it amounts to the essential beauty of contemporary liberalism. It is a conception to which everyone can subscribe, because everyone has the same moral capacity, and the same irrevocable moral value; if each were to act truthfully and in good faith then they would have to admit that their own conception of the good is reasonably contestable and that, as a matter of justice, each person should be allowed to choose and live their own conception. This would occur within a broader system of rights which recognise the moral value of each person and reasonable variations in conceptions of the good.

\footnotetext{
${ }^{68}$ Rawls, op cit, above note 5, p118.
} 
This simple structure of a liberal conception of justice is both strongly intuitively appealing and is remarkably cogent and logically sound. Its major flaws do not relate to the system derived from the priority of the right, but rather from criticisms of the priority itself and the assertions and beliefs that support it. This system is dependant on the idea of a real and effective distinction between the right and the good, and agreement on the nature of their relationship, namely one of absolute priority.

The contrast between the right and the good is one that is familiar in moral theory. It can be described in terms of the distinction between behaviour that is self-regarding and behaviour that is other-regarding, altruistic or sympathetic. However, Rawls' characterisation of this basic idea is more questionable. He treats the good as expressing a consequential material value that is contingent on the reaction of the valuer, or the meaning and value that person gives it. In this sense the good is personal and revisable, an assessment that can easily give way to the idea that conceptions of the good are ephemeral and spurious in any context broader than the individual who holds them. The right, by contrast, is indicative of an intrinsic and, therefore, necessary moral value that can be perceived by anyone who faithfully commits his or her moral abilities to its assessment. To Rawls, then, the good is contingent and morally arbitrary, and naturally subordinate to the right, which, in turn, is the common and true representation of moral value.

In many ways, there seems to be a plausible distinction between self-interested and moral behaviour. Rawls, however, gives this intuitive duality meaning that it cannot plausibly bear. He joins self-interest to our personal conceptions of the good life, our understanding as to how it is best to live, and he characterises these conceptions as being matters of personal preference, which have no meaning beyond our individual acceptance of them. In contrast, he equates the right with morality, and he characterises it as being rationally correct, singular and universal. According to this picture, varying conceptions of the good represent valid human diversity, whereas the right can always be the subject of rational examination and agreement.

The effect of this approach is to downplay actual moral difference and dispute, implicitly treating these as merely indicating the absence of rational analysis. It 
ignores the potential for the good to play a fundamental role in moral theory. It also assumes that our conceptions of how it is best to live do not have any moral or communal content, and, so, are not morally relevant in any significant way. It is entirely plausible that matters of both the right and the good play a substantial role in our moral lives, so that there is no clear and absolute priority of one over the other. Rawls' cleaving of right from good, his equating of this with moral and welfare concerns, and his characterisation of one being morally fundamental and the other morally arbitrary, seems unconvincing. The structural importance of this distinction brings into question much of Rawls' theory.

This position on the right and the good is then used to justify, both, the contention that justice should be conceived without reference to considerations of what is good and, also, that the liberties that follow from this conception should never give way to such considerations. Will Kymlicka questions whether these two uses are consistent with each other. He argues that

\begin{abstract}
[i]n discussing the definition of the good, Rawls says that there should be a priority of the right over the good, in the sense that our legitimate entitlements should not be tied to the promotion of any particular view of the good life. But this "antiperfectionist" sense of the priority of the right over the good has nothing to do with the first, "deontology versus teleology" sense of that priority. ${ }^{69}$
\end{abstract}

He goes on to suggest that these do not in fact concern the priority of the right over the good and do not reflect a single contrast in the way Rawls claims. Rather, they reflect two very different concerns, so that "there is not a single choice to be made here, but a range of possible positions that connect the two issues in various ways". ${ }^{70}$

There are significant links, both theoretical and structural, between Rawls' two uses of the priority of the right. The idea that liberty cannot be traded for welfare is a result of excluding conceptions of the good from the substance and justification of the theory, and both involve a seemingly similar subordination of the good to matters of justice. Yet Kymlicka is correct in suggesting that the two express different moral ideals. The exclusion of conceptions of the good from discussions of justice does not correspond to the subordination of matters of welfare to the basic liberties. The reasoning between the two is quite distinct: one is based on the idea that moral

\footnotetext{
${ }^{69}$ Kymlicka, op cit, above note 62, pp187-188.

${ }^{70}$ Ibid, p188.
} 
judgments should not be clouded by individual bias; the other, that an individual's rights should not be abrogated for the material benefit of some other person or group. It is only Rawls' broad-brush approach to the right and the good that make the assertion of its two separate principles seem correct. Kymlicka's criticism illustrates one of its most basic flaws: while it has the virtues of being strongly intuitive and extremely simple and neat, further inspection reveals it to be incoherent and unconvincing, and potentially at odds with reasonable moral beliefs.

It is the neatness of Rawls' broad-brush approach, as it fails to register the complexities of our moral lives, which underlies the seemingly flawless liberal structure of universal rights and common moral thought. John McNaughton criticises this aspect of the priority of the right:

\begin{abstract}
As long as ethics and justice, in particular, continues to be viewed and defined in terms of these classical philosophical distinctions, those of what James called "intellectualism" (i.e., right vs. good, is vs. ought, etc; as Dewey pointed out, to set up an exhaustive dualism presupposes, often wrongly, that the entities are entirely separate to begin with), inconsistencies... are bound to appear. The complex psychological, motivational, biological, and cultural patterns woven into the exquisite tapestry that is man and his actions are far too sophisticated to be reduced exclusively to particular concepts such as those of right and good. A proper delineation of justice requires as its necessary propaedeutic an adequate description of man IN [sic] his ethical situation, his concrete relationships to himself, to others, and to the world about him. ${ }^{71}$
\end{abstract}

McNaughton is arguing that justice is no less complicated in structure or substance than people themselves, and that Rawls' reliance on the distinction between the right and the good is an over-simplification that can only distort and limit his conception of justice.

Although it is difficult to rule out completely the possibility of a simple structure of morality, this idea seems a strong one. It might be argued in reply that the distinction between the right and the good, although not illustrative of the whole of justice, still has some explanatory or prescriptive power and, so, some role in moral theory. However, this is not the approach taken in A Theory. There, the distinction is applied without limitation, although often implicitly, to virtually every aspect of the justification and substance. This being the case, its failure brings into question much of the theory. The nature of this failure is such that our intuitive agreement with the

\footnotetext{
${ }^{71}$ J McNaughton, 'John Rawls and the Conflict Between Right and Good' (1980) Auslegung 270, pp277-278.
} 
elements of a potential reflective equilibrium would be undermined. The veil of ignorance, for example, seems a plausible tool for removing self-interested considerations from the original position. If the good has morally valuable content, however, this approach seems to undermine the intuitive value of the veil, and of the fairness of the original position.

The doubt as to the priority of the right over the good, then, should be kept in mind in assessing the value of the various parts of the substance of Rawls' theory. This is the final task in applying the methodology of uncertainty, examining the original position and the two principles of justice as the expression of the justification of the theory. In doing so, the problems identified in relation to the justification should also find expression, along with any criticisms that relate to only the theory's substance. Examining the substance of $A$ Theory involves looking at the conditions and assumptions, whether explicit or implicit, placed on the original position; considering the actual derivation of the two principles from those conditions; and, finally, considering the two principles independently as being intuitive expressions of our sense of justice.

\subsection{Implied conceptions}

The original position is supposed to be, in the words of Rawls, "the appropriate initial status quo, and thus the fundamental agreements reached in it are fair". ${ }^{72}$ This is achieved by employing conditions and assumptions that are intuitively accepted as being strongly moral, and that will necessarily lead to just results. Sandel makes the point that:

the conditions of the original position cannot be so immune from actual human circumstance that just any assumptions producing attractive principles of justice would do. Unless the premises of such principles bear some resemblance to the condition of creatures discernibly human, the success of the equilibrium is, to that extent, undermined. ${ }^{73}$

Because the intuitive response to the conditions of the original position must be common between people, it seems reasonable that they must in some way resemble

\footnotetext{
${ }^{72}$ Rawls, op cit, above note 5, p11.

${ }^{73}$ Sandel, op cit, above note 4, p43.
} 
actual human circumstances, as Rawls' theory in fact does. However, recognition is more important as a standard than resemblance; the conditions of the original position must be recognisable by everyone as being morally important. Their resemblance to our actual circumstances will be necessary as a matter of practicality, because intuitions will naturally tend to relate to what is actually the case. But this will only be relevant insofar as the moral content of the situation is capable of being recognised and accepted by all. Rawls attempts to formulate conditions that are effective while being the weakest possible, so that this agreement might be achieved in relation to a situation that is constrained in a morally meaningful way. ${ }^{74}$ However, the weakness of these conditions has been strongly doubted, and they have been described both as "strong and far-reaching", and not "either weak or innocuous or uncontroversial."75

This relates to my earlier criticism of Rawls' intuitionism. It was concluded that he fails in adequately explaining and justifying his use of intuitions, but that this would not exclude entirely the possibility of his approach being valid. Looking at the conditions of the original position involves looking at intuitionism in action, as it is expressed in the substance of the theory. The problem that we might have predicted from the earlier discussion is that Rawls presents a number of seemingly plausible conditions as being morally appropriate, and potentially agreeable, by those who apply only their moral judgment to their assessment; commentators respond to these by arguing they are actually highly contestable, and demonstrate Rawls' implicit bias. The veil of ignorance, the requirement of rationality and the equality implied in the original position each have some plausible moral content, at least to a person who shares some of Rawls' moral beliefs. Yet each can be criticised as being contestable in a way that indicates more than that the people contesting them are not applying their moral judgment in the prescribed manner. The veil of ignorance, for example, is supposed to remove information about morally arbitrary considerations, such as a person's material self-interest and their conception of the good, from any decision made in the original position. Yet this, as has been said above, rests on the idea that a conception of the good is morally inferior to the right, either in the sense of the good of any person or group, or the aggregate good of everyone. Any doubts that the distinction between the right and the good can be meaningfully drawn so as to leave

\footnotetext{
${ }^{74}$ Rawls, op cit, above note 5, p510.

${ }^{75}$ Nagel in Daniels, op cit, above note 1, p7.; Sandel, op cit, above note 48, p65.
} 
the morally relevant on one side and the morally inferior on the other, may mean that the agreement Rawls envisages is not reasonably possible.

The most prominent and persistent of these criticisms deal with the conditions together. They claim to find a structure implicitly supporting the original position, which runs contrary to Rawls' explicit theoretical foundations, by incorporating some hidden conception of the good, or some conception of the person that is patently liberal and questionable. William Galston, for example, identifies in the original position an implicit theory of the good, which assumes "the worth of human existence, the worth of human purposiveness and the fulfilment of human purposes, and the worth of rationality as the chief constraint on social principles and social actions." ${ }^{, 76}$ This theory incorporates ideas of rationalist humanism, which reflect the links between liberal theory and the Enlightenment. ${ }^{77}$ Although this assumption may seem inconsequential, Galston argues that the reliance of the original position on it reveals that $A$ Theory is not independent of any conception of the good, but rather promotes one type of conception over another: namely those that do not "preach the superiority of specific types of character and ways of life" over those that do. ${ }^{78}$ In other words, the veil of ignorance restricts knowledge about individual conceptions of the good, while the condition of rationality and the general set-up of the position itself impose such a conception.

This conception of the good is weak in that it depends on the general idea of human purpose rather than any narrow and more obviously personal ends, and, so, is no less plausible that other parts of $A$ Theory. Yet, permitting its role in the original position would require justifying why this conception is acceptable where others are morally arbitrary. Without this justification, it seems that Rawls' understanding of the good, in the words of Galston, "has the appearance of an arbitrary arrangement of convenience rather than a principled position", and that the liberal outcome of the original position seems dependant on its hidden liberal assumptions, rather than revealing new and common moral truth. ${ }^{79}$

\footnotetext{
${ }^{76}$ W A Galston, 'Defending Liberalism' (1982) 76(3) American Political Science Review 621, p625.

${ }^{77}$ Ibid, p626.

${ }^{78}$ Ibid.

${ }^{79}$ Ibid.
} 
Nagel explores the same area in looking at the thin theory of the good that Rawls attributes to people in the original position, namely their rational desire for primary goods. He then considers whether this limited conception undermines the remainder of the theory.

What Rawls wishes to do, by using the notion of primary goods, is to provide an Archimedian point, as he calls it, from which choice is possible without unfairness to any of the fuller conceptions of the good that lead people to differ. A theory of the good is presupposed, but it is ostensibly neutral between divergent particular conceptions, and supplies a least common denominator on which a choice in the original position can be based without unfairness to any of the parties. ${ }^{80}$

Nagel's characterisation of the implicit conception of the good is justifiable in that it is a minimal conception that is neutral as between more substantive beliefs. But this approach, Nagel argues, is not tenable. The problem is that, although the conception seems a weak one, "it depends on a strong assumption of the sufficiency of that reduced conception for the purposes of justice." ${ }^{81}$ In other words the conception is so heavily relied upon in Rawls' theory that it magnifies the non-neutrality of his thin conception of the good.

\footnotetext{
The suppression of knowledge required to achieve unanimity is not equally fair to all the parties, because the primary goods are not equally valuable in pursuit of all conceptions of the good. They will serve to advance many different individual life plans, ... but they are less useful in implementing views that hold a good life to be readily achievable only in certain well-defined types of social structure, or only in a society that works concertedly for the realization of certain higher human capacities and the suppression of baser ones, or only given certain types of economic relations among men.... The original position seems to presuppose not just a neutral theory of the good, but a liberal, individualistic conception according to which the best that can be wished for someone is the unimpeded pursuit of his own path, provided it does not interfere with the rights of others. ${ }^{82}$
}

Galston and Nagel both examine the conditions of the original position and find some hidden bias that undermines the status and value of the two principles that result. The difference in approach is that, while Galston considers the broader implications of rational purposefulness, Nagel focuses on a particular expression of the thin conception of the good in relation to primary goods. The non-neutrality of the original position between individual and communal goals is the result of the latter, and is an important practical consequence of this argument.

\footnotetext{
${ }^{80}$ Nagel in Daniels, op cit, above note 1, p8.

${ }^{81}$ Ibid, p9.

${ }^{82}$ Ibid, p10.
} 
Joseph Raz repeats this argument, and identifies that this non-neutrality is not limited to non-individualistic goods, but rather applies to "[a]ll conceptions involving the cultivation and satisfaction of the so-called expensive tastes" ${ }^{\text {}} 83$ But he agrees that nonindividualistic goals will tend to be expensive since "their realization depends on the cooperation of others, and they will take some convincing to come round to that agent's point of view." 84 This suggests that the original position is averse to resourceexpensive rather than non-individualistic goods.

This argument weakens Nagel's view in two ways. First, because it focuses on resource-expensive goods, it suggests that there could be some communal action that is not expensive, and in fact is favoured by the original position. It is quite possible that some such action could occur without the cost of convincing, and could involve cooperation, mutual satisfaction and even substantial economies. Second, it accords with the basic intuition that, in a world of scarce resources, people should to be responsible for the cost of their actions.

Raz's approach seems to miss some of what Nagel is claiming - that the original position presupposes a conception of the good that is inherently individualistic and, so, at odds with non-individual behaviour. In this way, the original position involves a discrimination against communal behaviour, not simply because it tends to be expensive, but because the original position is not easily amenable to groupdependant conceptions of the good. Raz's view is correct, that non-individual goals tend to come within the broader group of expensive goods; but, this view is incomplete. It suggests that, if making the distribution dependant upon the resourcecosts of conceptions of the good is valid, then Rawls' approach achieves neutrality between these various conceptions. In this situation there would be no discrimination against communal behaviour beyond the legitimate idea that the resource costs of that behaviour should be borne by those people who choose to adopt it.

However, Nagel is saying more than this: even if this approach is in this sense neutral between goods, the underlying system, including what 'goods' means in this context, is biased in favour of individualistic behaviour. It is possible to identify two

\footnotetext{
${ }^{83}$ J Raz, The Morality of Freedom (1986) p119.

${ }^{84}$ Ibid, p120.
} 
arguments here: that the use of the thin conception of the good in relation to primary goods involves a potentially arbitrary standard, i.e. the moral relevance of the resource-cost of a person's conception of the good; and, that the system in which this standard is embedded is constituted in terms of the individual and, so, will tend to not deal fairly with non-individual conceptions of the good. The difficulty is that this inherent bias is manifested through the resource-cost standard. However, the use of resource-cost itself is not in itself important here, since any standard the system uses to allocate resources will tend to reflect that system's bias.

Rawls has replied directly to this criticism of the individualistic bias of the original position. He argues that Nagel is mistaken in focussing on the neutrality of the original position, and that it is inevitable that some conceptions of the good will be favoured over others.

The most that can be expected, then, is that the original position be fair to the parties (conceived as moral persons). Even this may be asking too much, since there may be no very clear notion of fairness for this case or, at any rate, no notion of perfect fairness. But it is still worth asking whether the original position is as fair as possible; or whether some other account of the original position would be more fair. ${ }^{85}$

Rawls suggests that the appropriate standard for the conditions of the original condition is that of fairness, rather than neutrality between different conceptions of the good. He asserts that the original position is the fairest approach, a conclusion that presumably depends to some extent on the intuition that people should be responsible for the resource cost of their conceptions of the good. To this extent, Rawls answers Raz's argument that the original position is biased against expensive conceptions of the good. In relation to the separate charge of a potential individualistic bias, Rawls goes on to assert that, although the original position is individual in nature, it is capable of dealing with non-individual conceptions of the good: "it is true that the theory of the good uses the notion of an individual's plan of life; but this does not imply that such plans must be individualistic". ${ }^{86}$ In fact, in the well-ordered society that the original position prescribes, Rawls envisages the flourishing of communal values and actions:

The basic liberties are not intended to keep persons in isolation from one another, or to persuade them to live private lives, even though some no doubt will, but to secure the right of free

\footnotetext{
${ }^{85}$ Freeman, op cit, above note 9, pp270-71.

${ }^{86}$ Ibid, p273.
} 
movement between associations and smaller communities. There is, to be sure, one collective aim supported by state power for the whole well-ordered society: namely, that it be a wellordered society, a just society wherein a common conception of justice is publicly recognized; but within this framework communitarian aims may be pursued, and quite possibly by the vast majority of persons. Other socially collective ends may well exist besides that of being a wellordered society; but these ends cannot be upheld by the coercive apparatus of the state. If socially collective communitarian aims could survive in no other way, why should we regret their demise, and consider the original position unfair and arbitrarily biased against them? ${ }^{87}$

Nagel's argument is that the original position reflects a liberal conception of the good, and, so, is biased against non-individual values. Rawls' response seems to be that communal values can flourish despite being embedded in an individualistic framework. Furthermore, he suggests that this is appropriate; otherwise the state would be artificially supporting these values. The essential difference, then, depends on whether it is sufficient for the purposes of justice to be concerned with nonindividual values only insofar as they are expressed through individual values.

The original position, as Nagel has noted, uses individual decision making as a proxy for thinking about matters of justice, and utilises the perspective of individuals who have no knowledge of any personal communal beliefs or affiliations, as these fall within the boundaries of their conceptions of the good. This approach has the virtue of being clean in the sense that it allows thinking about justice that is conceivably unanimous and potentially universal. However, this is achieved at the expense of characterising people only in terms of their individual interests and expunging any communal tendencies. It is true that these can then be expressed within the boundaries of the resulting principles and that, in fact, individuals in the original position would be closely concerned with the freedom of individuals to express their communal values, but the fact remains that this is determined from an individualistic framework.

The extent to which this undermines the results of the original position must ultimately depend on the conception of individualism and collectivism, and the relationship of this to justice. This can be expressed in relation to the conception of the person used in the theory and, in particular, the relationship between a person and their normative and moral beliefs. An examination and criticism of Rawls' conception of the person is the basis for Sandel's Liberalism and the Limits of Justice. In it he comments that Rawls' liberalism

${ }^{87}$ Ibid, p281. 
avoids reliance on any particular theory of the person, at least in the traditional sense of attributing to all human beings a determinate nature, or certain essential desires and inclinations, such as selfishness or sociability, for example. But there is another sense in which this liberalism does imply a certain theory of the person. It concerns not the object of human desires, but the subject of desire, and how this subject is constituted. ${ }^{88}$

Rawls' conception of the person is unusual in that it focuses on the subject him or herself, or, in other words, the identity and capacity common between people, rather than on the nature of their preferences or desires. This means, Sandel argues, that people are antecedently individuated, that they are viewed primarily as morally capable individuals who can be separated from their subsequent beliefs or preferences, and, thus, are "non-contingent and prior to any particular experience". 89 With this conception the self "is distinguished from its ends - it stands beyond them, at a distance, with a certain priority - but it is also related to its ends, as willing subjects to the objects of choice." 90 This represents a deontological self, whose identity is independent from his or her "interests and ends and ... relations with others." 91

This conception of the person clearly reflects Rawls' priority of the right over the good in that it emphasises the common nature of our moral capacity, while distancing us from our personal desires or ends. In this context it seems natural for those in the original position to be shorn of their conceptions of the good. Further, the liberal outcome of the original position seems equally acceptable in that it states that there is a system of overarching freedoms, corresponding to our common moral sense, which allow us to express our individual values insofar as this is consistent with the rights of others. These moves make sense because people are only contingently related, and so stand apart from their ends. Given the difficulty already discussed with Rawls' priority of the good, this approach must raise questions, some of which are expressed by Sandel:

One consequence of this distance is to put the self beyond the reach of experience, to make it invulnerable, to fix its identity once and for all. No commitment could grip me so deeply that I could not understand myself without it. No transformation of life purposes and plans could be so unsettling as to disrupt the contours of my identity. No project could be so essential that turning away from it would call into question the person I am. Given my independence from the values I

\footnotetext{
${ }^{88}$ Sandel, op cit, above note 48, p10.

${ }^{89}$ Ibid, p49.

${ }^{90}$ Ibid, p59.

${ }^{91}$ Ibid, p55.
} 
have, I can always stand apart from them; my public identity as a moral person 'is not affected by changes over time' in my conception of the good. ${ }^{92}$

The basis of Sandel's criticism, then, is that Rawls' liberal project implicitly excludes that individuals can be constituted by their desires and ends. This downplaying of the good is at odds with a more communal conception of the person, whereby our beliefs, culture and goals form part of who we are, and from which we cannot sensibly be separated for the purposes of justice.

The underlying difference between this now-common theme of communitarian argument, and Rawls' system of liberal justice, seems to concern the relationship between the individual and, what Rawls would call, their conception of the good. It is not possible here to explore exhaustively this difficult relationship, but it is important to identify the implicit conceptions of the person and of the good that lie beneath Rawls' system of justice. That these conceptions are themselves contestable and, it is argued, reflect Rawls' own perspective rather than a universal truth, calls into question the purported universality of the whole system. This issue exemplifies the potential for bias in a moral theory, and the difficulties with the priority of the right over the good as an organising principle; it also highlights the danger of using intuitionism as a moral criterion where the system being assessed is structurally clean and quite plausible. Ultimately, no firm decision on the nature of the person, and the true relationship between individuals, norms and justice, can be made here, but the very fact of the argument and the nature of the disagreement undermines Rawls' assertion that his theory is the best of those available.

\subsection{Two principles of justice}

The final part of Rawls' theory concerns the two principles of justice. As part of the reflexive process these should be assessed from two perspectives, namely as being validly derived from the original position, and as being independently plausible and appealing as principles of justice. The goal here is not a perfect coincidence of the intuitive plausibility of the assumptions of the original position and of the principles

\footnotetext{
${ }^{92}$ Ibid, p62. Internal quote is of Rawls.
} 
themselves, but rather a coherent compromise between the two, characterised by reflection and adjustment. The assumptions of the original position have already been explored, which leaves for examination the derivation of the principles and their intuitive value.

The derivation of the liberty principle depends on the people in the original position, in ignorance of their particular conceptions of the good, deciding to implement an equal liberty for each to pursue these conceptions. Of course, this structure of the individual pursuit of the good, as embedded within a moral system of liberties, is reflective of Rawls' priority of the right, and, so, is subject to the same criticism, as well as the difficulty of applying restrictions on the original position that would produce a unanimous outcome. Putting aside these problems, the derivation of the liberty principle itself does seem quite reasonable. Although there are some arguments with regard to material equality, and also the makeup of these liberties, the idea that, once divorced from our self-interest and personal goals, we would tend to favour an equality of liberty, is quite compelling. In relation to this principle, at least, there seems to be the possibility of coherence between what rational people in the original position would do, and what seems just.

This quality is not present, however, in relation to the difference principle. As Brian Barry has put it, "[n]o other aspect of Rawls' theory has attracted more commentary than his effort to show that the difference principle can be derived from the original position as he specifies it, and it is, I think, safe to say that no other aspect of the theory has met with such uniform rejection." ${ }^{\text {93 }}$ In fact, this criticism amounts to a vast literature that cannot be fully explored here, although some general and representative arguments can be motioned, both in relation to the derivation of the difference principle and its intuitive value as part of a reflective equilibrium.

The difference principle is agreed to by those in the original position on the grounds that it approves of social and economic inequalities that are to everyone's advantage. ${ }^{94}$ A heuristic that is often used to describe the outcome of the difference principle is the maximin rule, which dictates that the choice between possible social

\footnotetext{
${ }^{93}$ B M Barry, A Treatise on Social Justice (1989), p214.

${ }^{94}$ Rawls, op cit, above note 5, p53.
} 
alternatives should maximise the position of the person who is worst off. ${ }^{95}$ The position of this person must be at least as good as in the position of equality. The decision to adopt the difference principle does seem, at first glance, to satisfy the constraints placed on those in the original position: they desire to increase their own level of primary goods, yet are unsure of their identity within the alternatives they are comparing. The adoption of the difference principle represents a conceivable reaction to this situation.

Hare has objected to the principle's derivation in claiming that it "does not appeal to me as in general a good one for choices under uncertainty. ${ }^{, 96}$ He argues that the original position, as constrained as it is, would not necessarily choose the maximin strategy, and that Rawls "has reduced the information available to them and about them so much that it is hard to say what they would choose, unless his own intuitions supply the lack". ${ }^{97}$ The basis of this argument is that, although the difference principle is a possible and not unreasonable result of the original position, it is not the only one. In other words, the conditions of the original position are not such as to lead to a unanimous agreement on the adopting principle. It is quite conceivable, for example, that some in the original position would choose a principle that causes a greater total material prosperity, to the detriment of some of the poorer citizens, and, so, effectively take the chance that they are not themselves one of the latter. It is not irrational, even in the Rawlsian sense of the term, to take this sort of risk. Under this view, the difference principle can properly be viewed as a reasonable, albeit conservative and risk-averse, response to the original position, and Rawls' explicit attempts to downplay the risk-aversion element of the principle as unconvincing. ${ }^{98}$

The literature aimed at demonstrating that the people in the original position might adopt some position other than the difference principle, including that of average utility, is prodigious. ${ }^{99}$ In addition to much abstract theorising, some have actually attempted to carry out experiments whereby subjects are placed in a situation analogous to the original position, and asked to choose between possible social

\footnotetext{
${ }^{95}$ Ibid, pp132-133.

${ }^{96}$ Hare, op cit, above note 54, p250.

${ }^{97}$ Ibid.

${ }^{98}$ Rawls, op cit, above note 5, pp72-73.

${ }^{99}$ E.g. M H Lessnoff, 'Justice, Social Contract and Universal Prescription' 28 (1978) Philosophical Quarterly 65, p69.
} 
alternatives. The experiment carried out by Michelbach et alia in 2003 is representative of much of the work in this area. The method used to simulate the original position and, then, interpret the results is quite ingenious.

Participants read a short description of a hypothetical society and are asked to imagine they are outside observers giving their advice to the society. In order to insure impartiality they are explicitly told they are not members of this society. They will evaluate different policies being considered by the society based on the effects they will have on the level and distribution of income as determined by "economic studies". 100

This involved looking at nine different income profiles and scoring each out of ten. The nine alternative societies represent each combination of low, medium and high equality, and low, medium and high efficiency. Each profile consists of five different representative levels of income. Put simply, more equality means a lesser gap between the levels, and more efficiency means a greater absolute income at each level. By looking at the scores given to each society, the subjects can be classified according to their attitude towards distributive justice, from strong egalitarian, to strong efficiencymaximiser, to Rawlsian maximin.

If the setup of the experiment does substantially mimic the original position, then the popularity of the maximin approach should have some relevance to the question of what people in the original position would actually do. The experimenters found that $18 \%$ of participants exhibited behaviour consistent with the Rawlsian approach. While substantial, this clearly falls short of the unanimity Rawls envisages in the original position. There are a number of potential differences between this position and the experiment. Most generally, as the experiment focuses on the difference principle alone, all primary moral and freedom-based issues are excluded.

In addition, the participants were explicitly told they were outsiders to the society, presumably to try to remove personal interest from the equation and, so, to simulate some of the informational limitations in the original position. It is conceivable that these sorts of differences distort the results of the experiment - perhaps people think differently in observing another society than one that will be theirs. Yet, the result that maximin is merely one potential distributive approach among a number of reasonable alternatives accords with the theoretical criticism of the derivation of the original

\footnotetext{
${ }^{100}$ P A Michelbach et al., 'Doing Rawls Justice: An Experimental Study of Income Distribution Norms' (2003) 47(3) American Journal of Political Science 523, p526.
} 
position, and is quite compelling. This result undermines Rawls' derivation of the difference principle as the potentially unanimous preference of those in the original position.

The difference principle is also criticised as being decidedly counter-intuitive. ${ }^{101}$ This point is illustrated by John Harsanyi's approach of applying the principle to personal decision-making:

Suppose you live in New York City and are offered two jobs at the same time. One is a tedious and badly paid job in New York City itself, while the other is a very interesting and well paid job in Chicago. But the catch is that, if you wanted the Chicago job, you would have to take a plane from New York to Chicago.... Therefore there would be a very small but positive probability that you might be killed in a plane accident....

The maximin principle says that you must evaluate every policy available to you in terms of the worst possibility that can occur to you if you follow that particular policy. Therefore, you have to analyze the situation as follows. If you choose the New York job then the worst ... possible outcome will be that you will have a poor job but you will stay alive.... In contrast, if you choose the Chicago job then the worst outcome will be that you may die in a plane accident. Thus, the worst possible outcome in the first case would be much better than the worst possible outcome in the second case. Consequently, if you want to follow the maximin principle then you must choose the New York job. Indeed you must not choose the Chicago job under any condition - however unlikely you might think a plane accident would be, and however strong your preference might be for the excellent Chicago job. ${ }^{102}$

This approach takes the difference principle out of its natural context, in that it applies to the different possible outcomes of a personal decision, rather than the different levels of material wellbeing within a society as a consequence of some principle of justice. In this way, the chance of dying in the plane crash corresponds to the chance of being poor as the result of an unfavourable principle. Clearly there is a conceptual difference between what might happen to you and what will happen to someone, and equally clearly, Harsanyi only reaches the result by assuming away the myriad of other possible outcomes of the decision: one could easily be killed in a car accident on the way to the New York job, or some malevolent being could kill the person because of their decision. The latter of these is ridiculous, but this is the point that Harsanyi is making, that a decision made according to maximin is highly irrational because it bases the decision on an outcome regardless of the chance of that outcome occurring. Applying it would mean that "you could never cross the street (after all, you might be

\footnotetext{
${ }^{101}$ E.g. Hare, op cit, above note 52; Gutmann, op cit, above note 43, p342.

${ }^{102}$ J C Harsanyi, 'Can the Maximin Principle Serve as a Basis for Morality? A Critique of John Rawls's Theory’ (1975) 69 American Political Science Review 594, p595.
} 
hit by a car); you could never drive over a bridge (after all, it might collapse); you could never get married (after all, it might end in a disaster), etc."103

If the difference principle is correspondingly irrational, then, it is because it involves focussing only on the poorest person, despite the small chance of any person in the original position actually turning out to be that person. In effect, Harsanyi notes, the difference principle means assigning a probability of one to the worst possibility, namely being the person who is the worst off. ${ }^{104}$ But the use of the maximin rule as a principle of justice is not quite so dire as this suggests. Focussing entirely on one person or outcome is, by itself, exceedingly irrational. But the worst-off person is not a random and morally arbitrary choice, but rather is the subject of the principle because he or she is particularly disadvantaged in relation to it. There is an important distinction between the difference principle as an individual decision-making procedure, and as a principle of social justice: the worst possible outcome is an independent contingency which has no relationship to the other possibilities, whereas the worst-off person is defined by their relationship to others, in that they have a lower material welfare.

It could be argued that the worst-off person is a representation of the effect or the extent of the effect of a decision, and, as such, is the valid focus for the concern of a just state. The intuitive value of the difference principle would then be based on the idea that the justness of a society depends on, or is reflected by, the way the worst-off person is treated, and that this implicitly considers the position of every person. This would mean that the difference principle could plausibly carry some normative value. But even if this is accepted, it does not mean that the two principles together amount to a complete and potentially universal system of justice, and, in fact, a weakened form of the irrationality Harsanyi describes still remains. Although the worst-off person may be relevant to justice, there are a number of other plausible considerations, such as income distribution, efficiency and desert, which could also be taken account of. The difference principle, in the system set out by Rawls, is characterised by an absolute unwillingness to trade off between different normative considerations and between different potential outcomes, and an abhorrence towards

\footnotetext{
103 Ibid.

${ }^{104}$ Ibid, p599.
} 
risk taking. All are subordinated to the single consideration of the worst-off and, as Harsanyi suggests, this fails to reflect the "great complexity and the unavoidable dilemmas of real-life moral situations". ${ }^{105}$

The final point about the difference principle concerns its practical ability to manage a system of inequalities. Putting aside the problems of identifying and measuring the inequality effects of various policies, there are inherent problems with the principle's effect. This touches on a difficulty relevant to all contemporary liberalism, namely how to reconcile voluntary exchange and co-operative and productive behaviour with a system of fundamental moral rights; and, in some cases, how to promote some form of material equality while permitting market behaviour that will inevitably and substantially upset it.

The concept of the difference principle relies on inequalities improving the positions of everyone, including the least advantaged. This implies a baseline that his or her position is compared with. Barry, in looking at the mutual co-operative aspect of Rawls' theory, identifies this baseline as the point of non-cooperation, or what each party would be able to obtain independently of others. ${ }^{106}$ In this way, the principles of justice seem to be compared to a non-cooperative state-of-nature type situation where this system is not followed. Although Rawls does express the benefit of the difference principle in terms of social cooperation, this does not mean that the appropriate baseline is that of non-cooperation. ${ }^{107}$ If the situation under the difference principle is to be compared to the situation without it, then the reasoning in the original position suggests that a better baseline would be that of equality. Since this equality is logically prior to the difference principle, and since the arguments in favour of it apply whether or not the difference principle is adopted, it does seem to be the position that would apply in the absence of the difference principle. This accords with Rawls' statement that the difference principle applies to inequalities "that work to make everyone better off in comparison to the benchmark of equality". ${ }^{108}$ It might also be argued that since the difference principle must ultimately be compared with other possible distributive principles, then these alternatives should form some part of

\footnotetext{
105 Ibid, p602.

${ }^{106}$ Barry, op cit, above note 90, p242.

${ }^{107}$ Rawls, op cit, above note 5, p88.

${ }^{108}$ Ibid, p131.
} 
a complex baseline position. This seems appropriate since the choice being made is not simply whether to apply the difference principle or not, but rather which of a number of possible approaches to apply.

Even if equality is taken as the appropriate baseline with which to compare the results of the difference principle, there is a further difficulty that relates to the operation of the difference principle over time. The difference principle ought to be able to incorporate some of the normal operation of a market. For example, voluntary exchange would often be permitted by the principle since all parties to the exchange are presumably made better off by it, or they would not have agreed. Over time this sort of exchange has the potential to substantially undermine the position of material equality, as some can make material gains by exchange and others lose out and consume their purchases. The passing of time would render the original distribution unrecognisable. This seems to undermine both the egalitarianism of $A$ Theory, and the arbitrariness of natural endowments, and supports a more conservative economic approach.

In defence of Rawls' position, the difference principle can only operate in a manner subordinate to the principle of liberal equality, and so must give way to an equality of opportunity, yet how the two positions can be reconciled, or what limitations this places on the market, is not clear. The basic difficulty is that the market will naturally lead to material inequality, and once this occurs, material equality only exists, much as with the original position, as some hypothetical starting point. In this light, the difference principle, with its focus on initial equality, seems hopelessly static, unable to deal with the dynamic shifts inherent in market operation, and quiet as to the nature and moral status of productivity and economic growth.

\section{Conclusion}

Rawls' basic aim in A Theory of Justice is to identify and explore a conception of justice that, he argues, is universally valid. One approach to achieving this would have been to identify some truth, about human nature or the nature of morality for instance, 
or, rather, a system of connected truths about a number of matters relevant to justice, and, then, derive a conception of justice directly from it. This conception would prescribe the best or highest mode of life, to which every member of society ought to aspire.

This perfectionist-type approach has proved problematic for a number of reasons, the first of which is the difficulty of identifying any system of truths that can be revealed to be universal, and yet is sufficiently restrictive to lead to a definite conception of justice. Rawls' approach is explicitly non-perfectionist. Although he aims at showing that his conception of justice is universally valid, this is in relation to a number of specified alternatives, and not to any possible conception. In attempting to achieve this universality he does not draw on specific moral or empirical truths, but, rather, on people's moral intuitions, using tools such as reasonableness, plausibility, approximations and incompleteness. The specific conception of justice Rawls promotes is characterised not as the result of a chain of truths and reasoning, but as a hypothetical decision-making situation that people are encouraged to recognise and explore. It should be no surprise, then, that this conception extols the virtue of liberty, namely that no single way of life is to be morally prescribed or politically favoured. Citizens within this regime would have, above all else, the power to determine their own direction and goals, and would, in furtherance of this, have command over a fair share of the resources of society.

The criticism of uncertainty is, at its sharpest, against perfectionist theory. It rejects the basic truths from which the theory is drawn, as well as the idea that such truths exist, and that they can be identified and used to show the correct conception of justice. Rawls' theory, being anti-perfectionist, is implicitly aware of these criticisms, and would be expected to be better able to respond to them. But, the examination that has been taken of his approach reveals a number of shortcomings, and, ultimately, undermines his assertions of universal value.

In general terms, there are two basic problems with A Theory of Justice. The first of these resembles the sort of problem faced by perfectionist theory, namely the correctness of an underlying truth or representation. The conception of the right and the good and the relationship between them amounts to a substantial assertion about 
the nature of our moral lives, and is used by Rawls to structure and justify much of his theory. Although plausible, Rawls ultimately fails to show that this distinction is the correct view of our moral behaviour and ought to guide moral theorising. This failure extends to the more fundamental uncertainty that no true characterisation of our moral nature can be made such that it could guide our deliberation to some universal moral truth. In this sense, Rawls' attempt leaves us none the wiser as to how moral theory might actually make general statements about morality that would allow conclusions to be drawn that might validly be described as universal.

The second basic problem with A Theory of Justice relates directly to its nonperfectionist approach. The use of intuitions as part of a moral deliberation that is supposed to reach a universal agreement is certainly one of the most interesting and compelling aspects of Rawls' theory. But, here, Rawls fails to fully justify his faith that such an agreement could ever be achieved. The criticism that the theory has faced suggests that the components of the proposed reflective equilibrium are, in fact, quite contestable, and that many who applied themselves diligently to their examination would not reach those same results.

Recognising the contestable nature of assertions about our moral intuitions leads to the concern that many of the results that Rawls presents are only particular expressions chosen from a number of equally possible alternatives, and that these indicate his own particular moral beliefs, rather than the true or correct approach. The possibility that Rawls' theory might be an expression of bias rather than universal moral beliefs is one that ought to be taken seriously in examining a theory that is explicitly intuitive, yet claims universality. Readers who share many of his beliefs might naturally be inclined to believe that these would be shared by anyone who properly addressed themselves to the issue. In this context one must also be wary of results that seem plausible. Many of Rawls' results seem convincing, yet they are not the only answer that the structure of the theory allows, and many might opt for some other result.

Ultimately, A Theory of Justice trades on the plausibility of many of its parts, but raises questions of bias and potential disagreement, and also is inimical to complexity in its results. The intuitions that Rawls presents as being those that we would agree to 
given proper examination, since they represent a potentially biased but plausible and broadly acceptable point of view, are naturally quite straightforward. Such results belie the potentially complex nature of moral issues, as well as the problems of adequately rationalising and expressing this complexity as part of a coherent moral theory. ${ }^{109}$

It is the nature of theory to simplify and rationalise, but this should not impoverish the theory relative to the full complexity and detail of the real world. Our moral capacities and intuitions are themselves seemingly complex, and are potentially plural and conflicting. What is important is that any moral theory is sufficiently representative so that it is capable of achieving its own aims. Although Rawlsian theory is nonperfectionist, and is aware of these sorts of difficulties, it does not overcome them so that its results might in some meaningful way be described as universal. In this sense, many of Rawls' plausible and simple results seem to do more than merely smooth out the bumps; rather, they present a view that is contestable and that ignores the inherent colour and texture of our complex moral lives.

${ }^{109}$ C.f. R D Gastil, 'Beyond a Theory of Justice' (1975) 85(3) Ethnopsychologie 183, p194; J W Chapman, 'Rawls's Theory of Justice' 69 (1975) American Political Science Review, 588, p592. 


\section{Anarchy, State, and Utopia}

\section{Introduction}

Anarchy, State, and Utopia is philosopher Robert Nozick's 1974 libertarian reply to the egalitarian conception contained in A Theory of Justice. It represents the primary recent exposition of a libertarian ideal, and the first response to Rawls that is itself a landmark in political theory. Both together have been described as having "arguably framed the landscape of academic political philosophy in the last decades of the twentieth century."1 Although these works prescribe principles of justice and fair distribution that are quite different, they are both part of a single thread of political theory; although it rejects the principle of material equality, Anarchy, State, and Utopia is itself a recognisably liberal conception of justice, as it is based on a strong theory of inviolable rights. Because it offers an alternative conception of liberal justice, and because it amounts to an important response to Rawls' approach, a careful analysis of Anarchy, State, and Utopia is necessary, both in relation to A Theory of Justice, and in its own right.

The most obvious difference between the two works is between their egalitarian and libertarian prescriptions. But for present purposes, these principles are not as significant as their approaches to justifying the respective conceptions of justice. Examining these will indicate whether Nozick has overcome any of the problems set out in the previous chapter, and the sense in which Anarchy, State, and Utopia represents a progression in liberal theory.

Any examination of the theoretical underpinnings of Anarchy, State, and Utopia is confronted with the difficulty that these are lacking. Nagel, in describing the work as being 'libertarianism without foundations', comments that:

\footnotetext{
${ }^{1}$ B H Fried, 'Begging the Question with Style: Anarchy, State, and Utopia at Thirty Years' 23(1) (2005) Social Philosophy and Policy 221, p221.
} 
[t]o present a serious challenge to other views, a discussion of libertarianism would have to explore the foundations of individuals rights and the reasons for and against different conceptions of the relation between those rights and other values that the state may be in a position to promote. But Nozick's book is theoretically insubstantial: it does not take up the main problems, and therefore fails to make the kind of contribution to political theory that might have been hoped for ... He has left the establishment of the moral foundations [of libertarianism] to another occasion, and his brief indication of how the basic views might be defended is disappointing. ${ }^{2}$

Nozick explicitly admits to leaving aside the moral and theoretical background to his theory, and to omitting a precise theory of the moral basis for the human rights that are at the very core of the work. ${ }^{3}$ These omissions are serious, and have never been addressed by Nozick. A recent re-evaluation of his theory less-than-charitably concludes that " $[w]$ here Nozick has not simply begged the question, the answers he provides are often internally contradictory or seemingly random with respect to any coherent moral vision.",

Nozick would be the first to admit that the style of his work is different from that of Rawls. Where A Theory is multi-stranded and theoretically robust, Anarchy, State, and Utopia is thinner, less reasoned and complete, and is more plainly a rhetorical statement of belief, rather than a technical statement of moral theory. For the purposes of this thesis this represents a particular concern, but it should be remembered that Rawls himself omitted various arguments fundamental to the theoretical structure of the theory, such as a full discussion of the relationship of our intuitions to moral theory and moral truth. Anarchy, State, and Utopia is, potentially, an interesting and relevant subject for this study. Its theoretical limitations may limit the discussion of some of its parts, and others may require some extrapolation from the available material, yet it still offers a potentially substantial contribution, and is certainly a significant part of the theory under examination.

The actual approaches of Rawls and Nozick are similar in that both utilise hypothetical worlds as part of their theoretical structures. However, these worlds differ both in detail and in the way that they are embedded in the justification of their respective theories. The two conceptions of liberal justice are structured differently

\footnotetext{
${ }^{2}$ T Nagel, 'Libertarianism Within Foundations' in J Paul (ed), Reading Nozick: Essays on Anarchy, State, and Utopia (1981), p193.

${ }^{3}$ See R Nozick, Anarchy, State, and Utopia (1974), pp9, xiv; S Scheffler, 'Natural Rights, Equality and the Minimal State' in Paul, op cit, above note 2, pp149-150, 152.

${ }^{4}$ Fried, op cit, above note $1, \mathrm{p} 222$.
} 
and have strategies of justification that are substantially different, and, in turn, imply different approaches to moral theory.

Anarchy, State, and Utopia has prompted less of a response than Rawls' work, and this tends to relate to the details of the workings of the theory, rather than its theoretical implications. As with A Theory, it makes sense to begin an examination of this work by setting out its basic structure on its own terms, without criticism, and then to engage in a detailed analysis of it, working outwards from its aims and justificatory strategy to its more substantive parts. This can then be concluded with a comparative analysis between the approaches of the two theories, in particular how each deals with the difficulties of uncertainty.

\section{Basic structure}

\subsection{Structure and justification}

Anarchy, State, and Utopia uses a hypothetical reasoning process to identify the boundaries of the legitimate state. This involves three arguments: the first characterises a state of nature and demonstrates how an ultraminimal state can arise out of it; the second shows how that ultraminimal state becomes a minimal state, which involves the addition of a scheme of resource redistribution. These two hypothetical moves are assessed according to an independent standard of legitimacy, such that, if each is shown to be morally legitimate, then the minimal state will have been shown to be legitimate. ${ }^{5}$ Nozick's final task is to show that any state activity that extends beyond the role of the minimal state breaches this same standard of legitimacy. In this way, the activity undertaken by the minimal state is justified, and all other activity is morally invalid. Because the redistribution undertaken by the minimal state is strictly compensatory, it is the last of these arguments that expresses the theory's libertarian flavour, in the sense of protecting individual rights over property. It is used by Nozick to demonstrate the illegitimacy of the sort of redistribution implied by Rawls' egalitarianism.

\footnotetext{
${ }^{5}$ Nozick, op cit, above note 3, p52.
} 
The basic form this theory takes is to characterise and assess the moral legitimacy of each of the ultraminimal, minimal and extraminimal states. Nozick employs a strict system of rights as the standard by which each move is assessed. These define the legitimate and illegitimate action of a state. ${ }^{6}$ They include the entitlement of a person to the products of their making or their labour, and, so, apply to acquired resources and wealth. These particular rights are fundamental to Nozick's scheme, and are explicitly borrowed from the work of John Locke. Government action that is inconsistent with this system of rights is illegitimate, which includes the forced contribution to the welfare of another. ${ }^{7}$ Importantly, these rights are not one aspect of a state's objectives that can be traded off with others to achieve some good, but, rather, act as inviolable constraints on its actions. ${ }^{8}$ Applying these rights strictly, Nozick argues,

reflect[s] the underlying Kantian principle that individuals are ends and not merely means; they may not be sacrificed or used for the achieving of other ends without their consent. Individuals are inviolable. ${ }^{9}$

Nozick adopts a position of Kantian deontology much the same as that of Rawls. The latter expresses this in the idea that some rights are so important that they cannot be traded off with welfare; with Nozick, the relevant rights must not be breached, which means that individuals cannot be forced to contribute to the welfare of others. The two are similar in that both assert the entrenched value of some set of rights against which the welfare of others cannot overcome or outweigh. Both mean that the central moral parts of the respective theories are constituted outside of any utilitarian trading-off, so that it cannot be argued that they are reducible to a utilitarian approach, or are some form of utilitarianism in disguise.

Yet these two expressions of deontology are part of two quite differently principled theories. The primary difference is not in the way in which the deontological idea is applied, but, rather, in the nature of the rights that are being enshrined: the set of equal liberties that would be agreed to in the original position for Rawls, and the set of

\footnotetext{
${ }^{6}$ Ibid, p29.

${ }^{7}$ Nozick, op cit, above note 3, p30.

${ }^{8}$ Ibid, p29.

${ }^{9}$ Ibid, pp30-31.
} 
Lockean property rights for Nozick. It is interesting to note here the significant difference between the fundamental moral content of the two theories.

Although Nozick does not fully explain and justify his particular system of rights, he does direct some attention to the foundations of their deontological nature. This is manifested in his searching for some aspect of human nature that explains why some moral rules must be applied strictly for the benefit of each individual, so that, in such matters, their interests should not give way to those of society. In doing so, he singles out our rationality, free will and moral agency, as well as our ability to regulate our lives in accordance with some overall conception that we have chosen as being significant. ${ }^{10} \mathrm{He}$ asserts that this issue "is concerned with that elusive and difficult notion: the meaning of life". ${ }^{11}$ In fact he identifies 'meaningfulness' as, potentially, representing that part of our nature that underlies these deontological rights. ${ }^{12}$

The inviolable nature of these rights links closely to voluntariness or consent in that it means that the state is restrained from forcing people to make contributions against their will. Nozick makes significant use of practical examples to illustrate many of the ideas of his theory, and one is particularly relevant here:

\begin{abstract}
Suppose some of the people in your neighbourhood (there are 364 other adults) have found a public address system and decide to institute a system of public entertainment. They post a list of names, one for each day, yours among them. On his assigned day (one can easily switch days) a person is to run the public address system, play records over it, give news bulletins, tell amusing stories he has heard, and so on. After 138 days on which each person has done his part, your day arrives. Are you obliged to take your turn? You have benefited from it, occasionally opening your window to listen, enjoying some music or chuckling at someone's funny story. The other people have put themselves out. But must you answer the call when it is your turn to do so? As it stands, surely not. Though you benefit from the arrangement, you may know all along that 364 days of entertainment supplied by others will not be worth giving up one day... Given these preferences how can it be that you are required to participate when your scheduled time comes? ${ }^{13}$
\end{abstract}

The idea that compelling someone to give up their time or some other resource amounts to morally illegitimate coercion is a persistent theme in Anarchy, State, and Utopia, and is in conflict with the Rawlsian notion that equality in liberty and primary goods is morally fundamental. It is the different sets of rights protected by each theory

\footnotetext{
${ }^{10}$ Ibid, p49.

${ }^{11}$ Ibid, p50.

${ }^{12}$ Ibid, pp50-51.

${ }^{13}$ Ibid, p93.
} 
that leads to these divergent results, and which imply differences in the approaches to justification and to moral theory.

\subsection{The state of nature and the ultraminimal state}

Nozick asserts that his theory is a fundamental explanatory account of politics, meaning that it is part of a potentially complete understanding of the political situation, derived from a non-political situation. ${ }^{14}$ This situation is one that is familiar in political theory, namely the state of nature, which simply describes the situation of people living prior to any political organization. Nozick reasons how the state might arise out of this anarchy, the idea being that, if an understanding of politics is derived from this fundamental pre-political situation, then it will, itself, carry some fundamental value, and the theory that expresses it, some universal legitimacy. ${ }^{15}$

Nozick's argument from the state of nature is hypothetical, but, in a sense, slightly different than that of the original position: the individuals who are the subjects of the latter are carefully engineered by Rawls so that their decisions are morally fair.

Nozick uses the state of nature not to show what would be chosen in an artificially restrictive situation, but, rather, to show how a state might arise out of a model of the situation faced by people in a pre-political context. The use of state of nature arguments is common in political philosophy and dates from Hobbes' Leviathan. Nozick adopts a characterisation that is explicitly Lockean:

Individuals in Locke's state of nature are in "a state of perfect freedom to order their actions and dispose of their possessions and persons as they think fit, within the bounds of the law of nature, without asking leave or dependency upon the will of any other man"16

The state of nature is not without restriction, since a person's actions can affect the ability of others to exercise their freedoms. This idea describes a set of standards that, even in the state of nature, ought not to be breached; as Nozick states, again quoting from Locke, "[t]he bounds of the law of nature require that no one ought to harm

\footnotetext{
${ }^{14}$ Ibid, pp6-7.

${ }^{15}$ Ibid, pp8-9.

${ }^{16}$ Ibid, p10 (quote from John Locke's Two Treatises on Government, book II, chapter 2, clause 4).
} 
another in his life, health, liberty, or possessions". ${ }^{17}$ Since the situation is prepolitical, however, there are no established institutional mechanisms to enforce these laws of nature or compensate for their breach, yet even in the state of nature there are abstract rights to self-defence and compensation, and to punish transgressors. ${ }^{18}$

In a state of nature it would be difficult for an individual to exercise these rights, particularly if the transgressor is strong. Nozick argues that this situation could lead to an informal system of mutual protection, whereby people help others enforce their rights, in exchange for the benefit of the assistance of others. ${ }^{19}$ As such relationships develop, mutual-protection associations would be born that would utilise their members in protection of their individual rights. Such associations would also adopt the role of settling disputes between members, since otherwise people might use their membership to avoid remedy. ${ }^{20}$ To settle these disputes some procedure must be employed. Nozick argues that the procedure most attractive to prospective members would be one that is non-arbitrary; one that actually determines whose claim is correct. $^{21}$ Equally, a mutual-protection association that only intervenes against nonmembers when the complaint is valid would be attractive in that reduces the cost of membership, by reducing the action the association would take. ${ }^{22}$

Nozick goes on to argue that conflict between rival associations will tend to lead to the creation of a single dominant protection association in any one geographical area. This consolidation results for one of two reasons: either one association consistently beats another (so that their determination of the issue becomes prevalent) and the members of the other association switch in order to have their rights better protected; or, the costs of conflicts between two systems lead to their merger, so that such conflicts are then dealt with within one institutional system. ${ }^{23}$

In each of these cases, almost all the persons in a geographical area are under some common system that judges between their competing claims and enforces their rights. Out of anarchy, pressed by spontaneous groupings, mutual-protection associations, division of labour, market

\footnotetext{
${ }^{17}$ Ibid. p10 (quote from John Locke's Two Treatises on Government, book II, chapter 2, clause 6).

${ }^{18}$ Ibid.

${ }^{19}$ Ibid, p12.

${ }^{20}$ Ibid, p13.

21 Ibid.

22 Ibid.

${ }^{23}$ Ibid, p16.
} 
pressures, economies of scale, and rational self-interest there arises something very much resembling a minimal state or a group of geographically distinct minimal states. ${ }^{24}$

Nozick describes the process which sees the rise of a single dominant protection association as an 'invisible-hand explanation', meaning that it occurs without conscious design. ${ }^{25}$ That the rise of the state is not dependant on any collective planning contributes to the parsimony of the theory. ${ }^{26}$

The last part of Nozick's argument is to inquire as to whether this dominant protection association satisfies the basic definition of a state. Put simply, the test for statehood he employs asks whether the association has a monopoly on the use of force. ${ }^{27}$ At this stage, however, the dominant protective association does not fully satisfy this requirement: although it may fairly be described as having a practical monopoly on force, there remains the possibility that some people would choose not be members, and, so, would enforce their own rights. ${ }^{28}$ So Nozick concludes that the dominant protection association does not appear to satisfy fully the requirements of a state. ${ }^{29}$ Instead, he argues that it resembles a kind of 'ultraminimal state'. Such a state

maintains a monopoly over all use of force except that necessary in immediate self-defence, and so excludes private (or agency) retaliation for wrong and exaction of compensation; but it provides protection and enforcement services only to those who purchase its protection and enforcement policies. People who don't buy a protection contract from the monopoly don't get protected. $^{30}$

\subsection{The minimal state}

Setting aside the issue of whether the dominant protective agency has the required monopoly on force, the ultraminimal state has been reached spontaneously and legitimately, without breaching anyone's rights or acting without their consent. The next move is to show that the actions of the ultraminimal state can be legitimately extended so as to create a fairer and more just system of government, which Nozick labels the minimal state.

\footnotetext{
${ }^{24}$ Ibid, pp16-17.

25 Ibid, p18.

${ }^{26}$ Ibid, pp20-21.

${ }^{27}$ Ibid, p23.

${ }^{28}$ Ibid, pp22-24.

${ }^{29}$ Ibid, p25.

${ }^{30}$ Ibid, p26.
} 
Nozick's reasoning from the ultraminimal to minimal state focuses on those acts of individuals that breach our natural rights. The illegitimacy of any state-sponsored breach is already established, but this does not suggest how a dominant protection agency ought to act in relation to the boundary crossing of an individual. Nozick begins by addressing the issue of whether the state should prohibit such action, or whether it should be allowed provided that the boundary crosser compensates their victim. $^{31}$

On one hand, if the latter is fully compensated then there is no good reason to prevent the boundary-crossing action; but, on the other, if the victim has not consented then the boundary should be respected. ${ }^{32}$ Given the importance Nozick places elsewhere on the strictness with which these rights are applied, the latter argument might be expected to prevail. Such a result has the additional virtue of avoiding the practical problems associated with implementing a system of compensating boundary-crossing, which would include determining the appropriate level of compensation in each case, as well as the possible fear and apprehension that would be caused by a system where a person's rights could validly be breached at any time without warning. ${ }^{33}$

These considerations lead easily to the conclusion that border-crossing ought always to be prohibited. However, Nozick himself adopts a more sophisticated position. He recognises that problems can prevent a person gaining consent for a proposed breach, and, so, permits some of those border-crossing acts where prior consent is impossible or very costly to negotiate, such as where the victim cannot be communicated with easily. ${ }^{34}$ These would be limited, as far as is possible, to those acts that do not create fear or apprehension, and would by implication include situations where consent would have been given were negotiation possible and practical. ${ }^{35}$

This conclusion seems a reasonable and practical response to individual bordercrossing, and is largely consistent with other parts of Nozick's theory. A greater

\footnotetext{
${ }^{31}$ Ibid, p57.

32 Ibid, p59.

${ }^{33}$ Ibid, pp 65, 66.

${ }^{34}$ Ibid, p72.

${ }^{35}$ Ibid.
} 
difficulty arises, however, when individuals undertake activities that will not necessarily breach the rights of another, but, rather, carry the risk of this occurring. This covers a wide range of activities, including those strongly beneficial to the parties involves and to society, and that involve only a slight risk of the relevant border being crossed. It seems clear that activity at this extreme ought to be permitted, while acts that will almost certainly lead to a breach ought to be prohibited, as if the breach were certain. The difficulty here is to draw the line between these different types of activities. ${ }^{36}$ The complexity and variation involved will not allow such a line to be concrete and straightforward, and both the nature of the activity and of the risk involved would be relevant, including the fear and apprehension that this caused were the activity permitted. ${ }^{37}$ As with the earlier example, this analysis would enable actions to be undertaken where the cost of gaining the consent of all those who might be harmed by the action would be prohibitively high, but where they might actually have agreed given the promise of compensation. ${ }^{38}$

An interesting issue raised here is whether those who have been prohibited from acting ought to be compensated for suffering that prohibition. There ought to be no compensation, Nozick argues, for not being allowed to play Russian roulette on others. ${ }^{39}$ But there are other prohibitions on activities that are important to many people, and so mean a genuine disadvantage that warrants compensation. ${ }^{40}$ Again, there is the need to draw a line in a complex and variable space, here, between that prohibited activity for which it is appropriate to compensate, and that which it is not.

The substance of Nozick's reasoning from the ultraminimal state, up to this point, concerns the actions of individuals that threaten to breach the natural rights of others. To deal appropriately with any such action it must be determined, firstly, whether it is to be prohibited, and, secondly, if it is, whether compensation is to be paid to the constrained person. This involves a complex factual analysis which depends on the nature of the activity involved and the costs of allowing it, including the fear of any person that they will be harmed by it, the risk that it will result in harm and the nature

\footnotetext{
${ }^{36}$ Ibid, p75.

${ }^{37}$ Ibid, p74.

${ }^{38}$ Ibid, p77.

${ }^{39}$ Ibid, p79.

${ }^{40}$ Ibid, pp81, 87.
} 
of the harm itself. Clearly this analysis is one of weighing up the consequences of allowing and prohibiting the action, and, so, is in the fashion of an economic costbenefit analysis.

However, this weighing up will be informed by the strictness with which the natural rights should be enforced. The main mechanism by which this strictness can be overcome is also economic in nature, namely that the transactions costs involved with acquiring ex ante consent to the possible breach would be prohibitive, but that such consent may have been attained were they not so. This keeps alive the idea that such rights ought not to be breached unless consented to, even if the consent will never actually be gained. It is also significant that these principles of prohibition and compensation are derived in the context of the state of nature, and do not depend on the existence of a functioning state. The prohibition that a dominant protection agency might enforce stems from the right of each person to defend themselves against having their rights breached by others, and the various types of compensation represent the just response to allowing and prohibiting various actions.

The next step in Nozick's reasoning from the ultraminimal to the minimal state is to identify the private enforcement of justice as one of those activities which risks breaching the natural rights of others. If people undertake to enforce their rights independently of the dominant protection agency, and their procedures are unreliable and dangerous, then they risk boundary-crossing against others, and so face potential prohibition. ${ }^{41}$ This means that, in effect, the dominant protection agency inherits the responsibility of ensuring that their members are treated fairly by others.

\footnotetext{
The dominant protective association may reserve for itself the right to judge any procedure of justice to be applied to its clients. It may announce, and act on the announcement, that it will punish anyone who uses on one of its clients a procedure that it finds to be unreliable or unfair. It will punish anyone who uses on its clients a procedure that it already knows to be unreliable and unfair, and it will defend its clients against the application of such a procedure. ${ }^{42}$
}

This power is derived from the right of each of its clients to resist the application of an unreliable procedure. ${ }^{43}$ It is through this process of resisting the application of

\footnotetext{
${ }^{41}$ Ibid, p89.

${ }^{42}$ Ibid, p101.

${ }^{43}$ Ibid, p102.
} 
other associations, and applying its own procedures, that the dominant protection association becomes what is recognisably a state.

Everyone has the right to defend against procedures that are in fact not, or not known to be, both reliable and fair. Since the dominant protective association judges its own procedures to be both reliable and fair and believes this to be generally known, it will not allow anyone to defend against them; that is, it will punish anyone who does so. The dominant protective association will act freely on its own understanding of the situation, whereas no one else will be able to do so with impunity. Although no monopoly will be claimed, the dominant agency does occupy a unique position by virtue of its power. ${ }^{44}$

Nozick argues that, because the dominant agency has the power to apply and enforce its procedures, and to exclude the use of the procedures of others, it has a de facto monopoly on the use of force and, so, satisfies the requirement of statehood. ${ }^{45}$ This completes his argument from state of nature to state, and shows how the latter can arise without the violation of any natural rights. ${ }^{46}$ It also describes a minimal state; one that will apply its own procedures of justice to determine disputes and will prohibit the use of any unauthorised methods, and one that will determine whether activity that threatens to breach the rights of another will be permitted and what compensation will result. These powers are built from only those rights granted voluntarily to the state by the individuals who possess them in the state of nature. ${ }^{47}$

As a coda to his theory, Nozick examines the merits of such a minimal state, independent of its legitimacy and the means of its development. Clearly it is not a utopia, in the sense of representing a single and uniform ideal of life. ${ }^{48}$ But, he argues, such a utopia would not be possible anyway: people are individual and have various criteria of goodness, and the idea of there being a single approach of life that can be manifested in the institutional structure and substance of a state does not recognise or support this enormous complexity. ${ }^{49}$

The conclusion to draw is that there will not be one kind of community existing and one kind of life led in utopia. Utopia will consist of utopias, of many different and divergent communities in which people lead different kinds of lives under different institutions.... Utopia is a framework for utopias, a place where people are at liberty to join together voluntarily to pursue and attempt

\footnotetext{
${ }^{44}$ Ibid, p108.

${ }^{45}$ Ibid, p109.

${ }^{46}$ Ibid, p114.

${ }^{47}$ Ibid, p118.

${ }^{48}$ Ibid, p297.

${ }^{49}$ Ibid, pp309-311, 313.
} 
to realize their own vision of the good life in the ideal community but where no one can impose his own utopian vision upon others. ${ }^{50}$

Such an open utopia has the advantages of being acceptable to most future utopians, regardless of their individual visions, and is "compatible with the realization of almost all particular utopian visions." ${ }^{, 51}$ It would also promote voluntariness and persuasion over coercion, as any person who requires the assistance of others to fulfil their own visions would have to do so through argument rather than the threat of force. ${ }^{52}$

Having described this utopia, Nozick then argues that the minimal state satisfies this description. ${ }^{53}$ The legitimate role of the minimal state does not coerce individuals into pursuing a particular way of life, but rather enforces efficient procedures of justice in response to breaches of the natural rights. Clearly this utopia incorporates some Rawlsian ideas. Most significantly, it excludes individual conceptions of the good from the state, and so offers a broad framework of rights within which individuals can act to achieve their own desires and ends. In A Theory of Justice this is reached through the priority of the right over the good. With Nozick, it is achieved through the strict application of natural rights, which a state coercing or imposing a particular way of life on individuals will breach. It is this theme that distinguishes Nozick's Anarchy, State, and Utopia as a distinctly liberal text, and one within the tradition starting with A Theory.

\subsection{Beyond the minimal state}

Having identified a viable minimal state that can arise without breaching the natural rights of any individual, and having only that power which has been voluntarily ceded to it, Nozick goes on to argue that no legitimate state can be more extensive. In particular, he identifies redistributive policies as being an improper activity for a just and legitimate state. ${ }^{54}$

\footnotetext{
${ }^{50}$ Ibid, pp311-312.

${ }^{51}$ Ibid, pp318-319.

${ }^{52}$ Ibid, p326.

${ }^{53}$ Ibid, p333.

${ }^{54}$ Ibid, p149.
} 
In making these claims, he employs an entitlement-based theory of distribution, which, he argues, is superior to the end-state and patterned principles used by such theorists as Rawls. Under this theory, a distribution is just if each person's holdings were gained either by a just acquisition, or the just transfer from someone else who was justly entitled. ${ }^{55}$

\begin{abstract}
Instead of rules of allocation which identify some human characteristic or propensity in accordance with which income and wealth are to be distributed (to each according to his need, ... merit, ... contribution), Nozick merely specifies those activities of appropriation and transfer which confer rights on people without reference to the relative condition or standing of their fellows. ${ }^{56}$
\end{abstract}

To achieve this Nozick examines how his system of natural rights applies to and affects matters of distribution. The concept of entitlement defines how people can legitimately acquire and deal with their holdings and, as with the standard of legitimacy that corresponds with the natural rights, is applied absolutely. This means that individuals can enforce these entitlements as against anyone else, and that any attempt to overcome these rules amounts to an illegitimate coercion.

The actual substance of the rules of entitlement, of acquisition, breach and rectification, are not fully detailed by Nozick; however, some points can be made. As with the natural rights, for example, it seems clear that one's entitlements can be voluntarily surrendered to another, so that exchange is legitimate.

The rules by which property is first acquired are brief, which is significant considering that all entitlements must ultimately stem from a legitimate acquisition. Nozick does discuss Locke's ideas of acquisition, in particular, how the mixing of labour and unclaimed capital can amount to a valid acquisition; but, these are not well-developed ${ }^{57}$ In relation to production generally, Nozick states that whoever makes something, provided they were entitled to any resources used in the process, is entitled to the end product. ${ }^{58}$ The acceptance of exchange and production at such a general stage in the reasoning does give Nozick's approach a capitalist feel, a point

\footnotetext{
${ }^{55}$ Ibid, p151.

${ }^{56}$ Paul, op cit, above note 2, p3.

${ }^{57}$ Ibid, pp174-178; Paul, op cit, above note 2

${ }^{58}$ Nozick, op cit, above note 3, p160.
} 
with which he seems comfortable. ${ }^{59}$ This leads him to summarise the distributive effect of entitlements, at first in detail:

From each according to what he chooses to do, to each according to what he makes for himself (perhaps with the contracted aid of others) and what others choose to do for him and choose to give him of what they've been given previously (under this maxim) and haven't yet expended or transferred. ${ }^{60}$

He restates this simply as: "From each as they choose, to each as they are chosen."

In support of this system of entitlements, Nozick explores the nature of different possible principles of distributive justice. He begins by distinguishing between historical and end-state principles. The former recognise that "the past circumstances or actions of people can create differential entitlements or differential deserts to things." 62 The latter are structural principles that apply in relation to one point in time. ${ }^{63}$ Nozick also applies the idea of patterned principles, where the resulting distribution "is to vary along with some natural dimension, weighted sum of natural dimensions, or lexicographic ordering of natural dimensions." ${ }^{64}$ These dimensions might include moral merit, usefulness to society, and IQ; in fact, most suggested principles are of the patterned type. ${ }^{65}$

Nozick's system of entitlements is an example of one distributive principle that is not patterned, in that, although several patterns run through the resulting distribution, there is no single or simple combination of natural dimensions by which the principle is defined. ${ }^{66}$ Even if some of the factors that contribute towards the overall distribution are patterned, such as marginal productivity, there are other factors involved that will not be reducible to this kind of 'natural dimension', such as the effect of gifts, charitable donations and bequests. ${ }^{67}$

\footnotetext{
${ }^{59}$ Ibid, p158.

${ }^{60}$ Ibid, p160.

${ }^{61}$ Ibid.

${ }^{62}$ Ibid, p155.

${ }^{63}$ Ibid, pp153-154.

${ }^{64}$ Ibid, p156.

${ }^{65}$ Ibid.

${ }^{66}$ Ibid, p157.

${ }^{67}$ Ibid.
} 
Nozick uses this characterisation of different distributive principles to criticise other approaches, including that of Rawls. To achieve this he uses what is probably the most recognised example from his theory, that relating to Wilt Chamberlain:

[S] uppose that Wilt Chamberlain is greatly in demand by basketball teams, being a great game attraction.... He signs the following sort of contract with the team: In each home game, twentyfive cents from the price of each ticket of admission goes to him.... The season starts, and people cheerfully attend his team's games; they buy their tickets, each time dropping a separate twenty-five cents of their admission price into a special box with Chamberlain's name on it. They are excited about seeing him play; it is worth the total admission price to them. Let us suppose that in one season one million persons attend his home games, and Wilt Chamberlain winds up with $\$ 250,000$, a much larger sum than the average income and larger even that everyone else has. Is he entitled to this income? Is this new distribution... unjust? If so, why? ${ }^{68}$

Nozick argues that the distribution prior to the basketball season was fair since it reflects some patterned principle, whichever a particular reader favours. This being the case, are not the voluntary payments to Wilt, and the resulting distribution of wealth (relatively strongly unequal in his favour), also fair? Anyone who agreed with the patterned principle that defined the initial situation, but argued that the outcome was unfair, would need to show how the consensual exchange was morally illegitimate. Given the voluntary aspect of this exchange, and the power that individuals have to use their fair resource allocation as they choose, showing that the result is not fair is a difficult task.

Nozick argues that this difficulty shows that both end-state and patterned principles cannot "be continuously realized without continuous interference with people's lives." ${ }^{69}$ In relation to the Chamberlain example, were the original patterned principle to prevail, then, either the contract with the basketball star must be prohibited, or there must be a redistribution that effectively undoes its result, to the extent that it is contrary to the particular pattern or patterns on which the principle is based. The same is true with end-state principles, since the contract would result in some deviation from the end-state position, just as it does from the patterns. This means that end-state and patterned distributive principles cannot operate over time in a manner consistent with contractual freedom and the voluntary utilisation of one's resources. This result is especially startling in relation to an egalitarian principle, since an egalitarian distribution would inevitably be destroyed by voluntary transactions. ${ }^{70}$

\footnotetext{
${ }^{68}$ Ibid, p161.

${ }^{69}$ Ibid, p163.

${ }^{70}$ Ibid, p164.
} 
Thus Nozick buttresses his system of entitlements by drawing on ideas of transactional freedom, and, in effect, extends his argument against a more than minimal state. Any theory which attempts the latter faces two problems: firstly, any non-consensual takings or transfer of resources by the government is contrary to our natural rights; and, secondly, any patterned or end-state distributional approach will inevitably be undone, unless the rights over resources are substantially abridged.

\section{Analysis}

\subsection{Universal aims and justificatory approach}

At its most basic, Anarchy, State, and Utopia is intended as a cogent defence of a libertarian state. This defence involves two separate tasks: justifying the minimal state, and showing the moral illegitimacy of a more extensive state. The first of these is based on the identification of state legitimacy with adherence to a number of natural rights; with the second, these rights are developed into a system of distributive entitlements that sets boundaries on legitimate resource holdings, and which nothing more than the minimal state can respect.

In effect, these two tasks represent responses to an anarchist position, and to alternative positive theories of social justice respectively. They are intended to be universal in effect; the standards of legitimacy they employ are not contingent on any particular social context, nor do they presuppose an existing set of unjustified beliefs. Yet, as with Rawls, in A Theory of Justice, Nozick is explicitly aware of some of the limitations his theory faces, and of some shortcomings of moral theory generally.

In the preface Nozick argues that works of philosophy should not be presented as "a finished, complete and elegant whole," and, instead, suggests that such works can be incomplete and can contain "unfinished presentations, conjectures, open questions 
and problems, leads, side connections, as well as a main line of argument." ${ }^{, 71}$ He goes on to characterise more certain philosophical approaches as "pushing and shoving things to fit into some fixed perimeter of specified shape."72

All those things are lying out there, and they must be fit in. You push and shove the material into the rigid area getting it into the boundary on one side, and it bulges out on another. You run around and press the protruding bulge, producing yet another in another place. So you push and shove and clip off corners from the things so they'll fit and you press in until finally almost everything sits unstably more or less in there; what doesn't gets heaved far away so it won't be noticed ... Quickly, you find an angle from which it looks like an exact fit and take a snapshot; at a fast shutter speed before something else bulges out too noticeably. Then, back to the darkroom to touch up the rents, rips and tears in the fabric of the perimeter. All that remains is to publish the photograph as a representation of exactly how things are, and to note how nothing fits properly into any other shape. ${ }^{73}$

Nozick recognises the complexity of moral theory: the difficulty of producing a consistent and complete system, fully and precisely detailed, that expresses the whole of some philosophical truth. Nozick's theory itself should be studied in the light of this admission, since the validity of any justificatory strategy, and so theoretical substance, depends on their being consistent with an acceptable model of moral theory.

It is clear that Nozick's theory, in order to be consistent with his views on philosophical activity generally, would not attempt completeness or finality. It is equally clear that Nozick is attempting to say something universal and meaningful in the area of political philosophy about actions of the state, albeit that the truth of this statement is incomplete and subject to development with political theory itself.

If conscious incompleteness and inconsistency are to be expected within the substance of Anarchy, State, and Utopia, then Nagel's criticism of it as being theoretically insubstantial and without foundations becomes less significant. ${ }^{74}$ It has been commented that

Nozick's explicit objectives for the book obviously fall short of providing the sort of "knockdown" argumentation for his position that we expect of the paradigmatic philosophical work. What he hopes to do is to lend plausibility to his libertarianism by deflating a variety of alternative positions, thereby leaving the field open to a possible successor ... Nozick aspires to elevate natural rights libertarianism to serious contention among competing political

\footnotetext{
${ }^{71}$ Ibid, pxii.

${ }^{72}$ Ibid, pxiii.

${ }^{73}$ Ibid.

${ }^{74}$ Above, note 2.
} 
philosophies. This aspiration is not ambitious enough to satisfy Nagel who consequently impugns the book for its neglect of foundational questions. ${ }^{75}$

This view of Nagel's assessment is appealing, but seems to identify Nozick's own aims in writing the book as the reason for its limitations, rather than being a reflection of the inherent difficulties of philosophical inquiry. This doesn't change the general point: the theory should not be indicted for its structural or substantial incompleteness, and, while its main principles and arguments should be viewed as part of a coherent theoretical system, this system does not attempt to be the final incontrovertible truth on the matter.

Even the recognition of this, however, does not completely refute Nagel's argument. Nozick's views on philosophy are not so generous as to be entirely unconcerned with justification or with avoiding glaring logical inconsistencies; such an approach would remove the possibility of any meaningful theory. The tension inherent in the justification of Nozick's theory is between accepting the limitations of philosophical activity - the possibility of fully expressing some ultimate, unchanging truth - and attempting to devise a theory that itself has some universal content and importance. The implicit task of his theory, then, is to make some legitimate response to these difficulties, and lend the substance of his theory some moral weight.

Were Nozick to succeed in this undertaking, his theory would achieve some moral value that is universal, but bounded or limited by the view of moral philosophy on which it is based. The idea of a bounded universality was raised in relation to $A$ Theory of Justice, and there are some underlying similarities between the approaches of Nozick and Rawls, such as the shared doubts as to the power of philosophical thought. Despite the basic similarities, the two aspire to different ideas of universality, corresponding with different conceptions of the role of moral theory, which result in quite different theoretical approaches. Rawls' approach is explicitly anti-perfectionist, and, so, as a matter of principle rejects the idea that there is a single ideal conception of life that political or moral theory is able to elucidate. His response to the limitations of moral theory ingeniously and importantly involves the application of rational thought in the original position, and our considered moral intuitions as part of a

\footnotetext{
${ }^{75} \mathrm{~J}$ Paul in Paul, op cit, above note 2, pp13-14.
} 
reflective equilibrium. This is developed into a logical structure that is both closely argued and well developed.

In contrast, the limitations inherent in philosophical inquiry lead Nozick to focus on a few general ideas, arguments and principles, and the central relationship between them. His political theory is self-consciously incomplete and is not presented as a consistent and true whole, but, implicitly, within the context of a tradition of moral and political thought and as part of the continuing development of ideas and beliefs. What can be assumed from the manner and tone of Nozick's theory is that the fact of being one step in the development of ideas, does not imply arbitrariness. What is being asserted is a truth, not the whole truth, but a truth that changes and potentially develops over time. Unfortunately, Nozick does not present an understanding of this truth, and is content with identifying the difficulties of moral philosophy and then building the substance of his theory. In terms of justification, his approach is less interesting than that of Rawls given its complete dependence on the necessary moral value of a system of infrangible natural rights. The power of the resulting theory will inevitably depend on the justification of the standard implied by these rights.

It is interesting to note the complexity of potential universalities. The assertion of a political theory to universality is not identical to any other such assertion, but, rather, has a complex nature that reflects the methodology of the theory, as well as implying a view of the role and power of moral theory generally. The recognition in a theory of some form of moral uncertainty will necessarily restrict the nature of the universality the theory can hope to achieve. This will flow into the justificatory strategy and the substance of the theory. Viewing Nozick's theory from the perspective of uncertainty, as with any other, involves looking at each of these parts in turn and inquiring as to their success in achieving the aims of the theory, as well as considering the way in which this represents a response to moral uncertainty.

Nozick's theory is founded in the hypothetical: it shows how a minimal state might arise without breaching our natural rights, and, then, that no more extensive state could act without breaching the system of entitlements developed from these rights. These arguments are buttressed by the utopian value of the minimal state, and by criticising alternative distributive principles on the grounds that they will either be 
corrupted over time, or must abridge some fundamental beliefs about the rights we hold over resources. The analysis of the theory will deal with each of these steps in turn, but must begin by examining the standard by which they are guided, namely the system of rights imposed over the hypothetical world.

\subsection{Natural rights}

The importance of the system of natural rights is reflected in the first passage of Anarchy, State, and Utopia:

Individuals have rights, and there are things no person or group may do to them (without violating their rights). So strong and far-reaching are these rights that they raise the question of what, if anything, the state and its officials may do. How much room do individual rights leave for the state? ${ }^{76}$

This question is the guiding principle of the theory: given that there are a set of rights that are so fundamental that even the state cannot legitimately breach them, what role can the state legitimately perform? The theory's validity rests on justifying this system of rights, and demonstrating both their inalienability and their relevance to justice.

A common criticism of Anarchy, State, and Utopia is that Nozick operates in an environment that is "theoretically inadequate", or, in a recent assessment, rather than elaborating or defending his ideas, he "begs or dodges the question." 77 This has been levelled, in particular, at his account of incontrovertible rights, which has been criticised variously as lacking in foundation, and as inadequately explained and explored. ${ }^{78}$

Nozick's argument in support of these rights is limited and, in tone, is impressionistic rather than precise and rationally compelling. He argues that they reflect a Kantian and deontological ethic, that the operation of fundamental rights respects the separate existence of persons, and the view of each person's life as an end. But this is

\footnotetext{
${ }^{76}$ Nozick, op cit, above note 3 , pix.

${ }^{77}$ Fried, op cit, above note 1, p226.

${ }^{78}$ E.g. Nagel, op cit, above note 2, p193.
} 
discussed rather cursorily, and has been fairly described as a vague allusion and opaque description. ${ }^{79}$ The remainder of Nozick's argument in favour of these rights centres on some fundamental ideas of free will, moral agency and meaningfulness that are "elusive and difficult" to deal with in a work of political theory. ${ }^{80}$

Given his general approach, and the nature of the subject on which he draws, the limited and impressionistic character of these arguments is not surprising. The issue is whether this can offer adequate support to what is essentially the central justificatory criterion of the theory; whether Nozick's explicit question-begging leaves enough of a foundation on which even a provisionally valid theory can stand; or, whether he is merely relying on the intuitive plausibility of fundamental and inexorable rights. This leads one to question of this foundation, to then examine the system of rights for more substantive omissions, and, finally, to ask whether the system thus revealed can validly play the role asserted by Nozick.

Apart from being vacuous, there have been a number of accusations levelled at Nozick's justification of these rights. Nagel has, in particular, criticised the underpinnings of their inalienability. Nozick builds these on the Kantian idea that the good of one person cannot be sacrificed for that of another:

\begin{abstract}
Why not ... hold that some persons have to bear some costs that benefit other persons more, for the sake of overall social good? But there is no social entity with a good that undergoes some sacrifice for its own good. There are only individual people, with their own individual lives. Using one of these people for the benefit of others, uses him and benefits the others.... To use a person in this way does not sufficiently respect and take account of the fact that he is a separate person, that his is the only life he has. ${ }^{81}$
\end{abstract}

The perspective here is from a strong sense of individuality: people are separate from one another; respecting this prohibits their good being sacrificed for that of others. This idea informs the system of rights that each person enjoys. Nagel has questioned this reasoning:

It is not clear how Nozick thinks individual rights derive from the fact that each person's life is the only one he has. He appears to draw the implication that a benefit to one or more persons can never outweigh a cost borne by someone else. This however is far too broad a claim for Nozick's purposes. It is both obviously false and unsuitable as a basis for constraints on the treatment of individuals... To make sense of interpersonal compensation it is not necessary to

\footnotetext{
${ }^{79}$ Paul, op cit, above note $75, \mathrm{p} 8$.

${ }^{80}$ Nozick, op cit, above note 3, p50.

${ }^{81}$ Ibid, pp32-33.
} 
invoke the silly idea of a social entity, thus establishing an analogy with interpersonal compensation. All one needs is the belief, shared by most people, that it is better for each of 10 people to receive a benefit than for one person to receive it, worse for 10 people to be harmed than for one person to be similarly harmed... and so forth. The fact that each person's life is the only one he has does not render us incapable of making these judgments, and if a choice among such alternatives does not involve the violation of any rights or entitlements, but only the allocation of limited time or resources, then we regard those comparisons as excellent reasons for picking one alternative rather than the other. ${ }^{82}$

Nagel here shifts the focus from the separateness of persons to comparisons of good. Nozick is stating, he claims, that the good of one can never outweigh the good of another, so that no person's good should be sacrificed for another. But, if this is the case, there is no way of meaningfully comparing the good of different people, even when rights or entitlements are not concerned.

This argument depends on reducing Nozick's position to the claim that interpersonal comparisons of the good are never possible, at least not in any meaningful way.

Nozick might reply that Nagel has confused the making of interpersonal comparisons with basing the moral permissibility of social or state actions on them. He might admit that most people would accept the good of ten people to have more moral value than the similar good of one, but argue that this does not imply that the good to which that one is entitled should be sacrificed to them.

The difficulty in analysing this claim is that, as Nagel suggests, it is not clear how a respect for the separateness of persons leads to inalienability. Nozick's reasoning is that people are separate, so that the good of each is also separate. This means that we should not make decisions based on the aggregate of their good, nor can we trade off between the good of different people, because this fails to respect their separateness. What is unclear is the nature of this failure. Nozick suggests that to trade off the good of one person for another is effectively to use that person; it does not respect the distinct importance and value of their good; a value that cannot be superseded by the value of another. Nagel takes from this that one person's good cannot be outweighed, in the sense of never being able to compare the good of one to the good of another. An alternative interpretation, however, entails that Nozick is not concerned with the comparison of values, but rather with their moral standing; that it is possible to

${ }^{82}$ Ibid, p197. 
meaningfully compare value, but that the distinct moral value of each prohibits one being used for the other.

Such a reply deflects much of Nagel's argument, but prompts further questions as to the source of the inalienability of rights. This stems not from the inability to compare the good of different people, but, rather, the moral inadmissibility of acting on any such comparison where matters of fundamental rights are involved. This is founded on the distinct moral value of the good of individuals, which should not be traded off with others. But the issue of why this is the case remains. Nozick needs to offer an account of moral value that both explains this distinctness of value and its moral consequences, and also that links this directly with the subjects of the fundamental rights. Even if a deontological ethic is accepted, the substance of the resulting rights needs to be explained. Rawls applies similar ideas of distinct value, but these do not extend to individual good; so, his theory is redistributive in a way that Nozick's could not be. The portrayal of philosophical inquiry as necessarily incomplete does not excuse the absence of this discussion, as it is fundamental to the justification of the remainder of the theory. Neither can intuitive appeal carry the burden of supporting an absolute moral standard.

Nozick does offer an additional argument in favour of these rights, based on the elusive idea of the meaningfulness of life. In fact, it is this idea that seems to imply the distinct value of individual good. ${ }^{83}$ This has been criticised by Samuel Scheffler:

[W] hat is important is that Nozick believes that the moral basis of rights has to do with the capacity to live a meaningful life. For then we may ask, what rights would such a basis be the basis $o f$ ? In other words, if the capacity to live a meaningful life is a uniquely valuable characteristic, and if we say that beings with this characteristic have rights, in virtue of which there are constraints on the way others must behave, then presumably the function of the rights is to safeguard the ability of beings with the valuable characteristic to develop it... [I]t seems clear that the alternative conception of rights is a much more accurate specification than the Lockean conception of the rights which people actually have. For the alternative conception assigns to each individual the right to a sufficient share of all distributable goods whose enjoyment is necessary to have a reasonable chance of living a decent of fulfilling life. Now whatever a meaningful life may turn out to be, it seems reasonable enough to suggest that any distributable good necessary for living a decent and fulfilling life (such as food) will also be necessary for a meaningful life.... If that is so, then the alternative conception of rights is clearly the correct one: for it alone insures that all the necessary material conditions for having a reasonable chance of living a meaningful life will be met. $^{84}$

\footnotetext{
${ }^{83}$ Ibid, p50.

${ }^{84}$ Scheffler, op cit, above note 3, pp158-159.
} 
Scheffler examines the link between the capacity to live a meaningful life, and the content of a conception of rights. He argues that, if rights are to be justified by this capacity, then the latter should inform the substance of the former; in particular, we would expect rights to protect and nurture our abilities to live meaningfully. Scheffler then asserts that this is best achieved, not by Nozick's system of natural rights, but by an alternative conception that champions the right to "a sufficient share of every distributable good whose enjoyment is a necessary condition of the person's having a reasonable chance of living a decent and fulfilling life" ${ }^{, 85}$ In other words, a better system of rights is one explicitly conceived to further the ability of individuals to live decent and fulfilling lives, which corresponds with the development of our capacity for meaningfulness.

Both Scheffler and Nozick take as the foundation for a system of natural rights the human capacity for meaningfulness, or some similar distinctly human ability ${ }^{86}$ From this, Scheffler derives a principle whereby each person has the right to a sufficient share of resources relevant to exercising this ability, whereas Nozick identifies meaningfulness as the source of the value of individual good, and so favours a principle where each person has the right to those resources legitimately acquired, which cannot be abridged for the good of others. These could also be compared with a third, Rawlsian approach, whereby the principle derived is of an equality of resources and opportunities to exercise them, in order for each person to have a fair opportunity to realise their capacity for individual fulfilment.

Each of these three conceptions of rights suffer from the same problem, namely that their foundations, in this case the capacity to lead a of meaningful life, don't necessarily define the rights they justify. In fact, each of these three conceptions, sufficiency, equality and non-abridgment, are all reasonable derivations from the fundamental capacity to lead meaningful lives. Each protects and nurtures this capacity in a substantial way: by ensuring each person has enough resources to have a reasonable chance of having a meaningful life; by ensuring an equal call on resources with which to have a meaningful life; and, by strongly protecting the resources to which each person is entitled against any appeal from the good of others. Each of

\footnotetext{
${ }^{85}$ Ibid, p153.

${ }^{86}$ Ibid, pp152-153.
} 
these has also its own intuitive appeal: the natural abhorrence of poverty and enslavement; the idea of a strict fairness of distribution; and the idea of strong property rights, and control over an individual's entitlements.

The point here is that the appeal to some general human capacity will not in itself indicate the correctness of one conception of rights, to the exclusion of all others. Such a capacity will inevitably be able to be expressed in a number of alternative ways, corresponding with different moral principles and expressions of moral value. Some of these conceptions may be better or more complete expressions of the capacity in question, and some that are initially appealing may turn out to be wrong, unrealistic or have unappealing or inconsistent consequences. But no conception can be the complete its moral expression. Nozick may emphasise the incompleteness and continuing nature of philosophical enquiry, but this discussion suggests something more: a contestability and openness that belies the assertions of universality implicit in his theory. It is simply the case that definitive moral theory cannot be strictly derived from such general ideas.

However, such moral-political theories are not merely attempts to express some human capacity in a system of rights; they each have additional justificatory aspects and principles that flesh out the structure and substance of the theories. Rawls uses the original position in reflective equilibrium to reach a result that is supposed to express, in a moral way, the rights appropriate to the circumstances of our existence. Scheffler himself is not arguing absolutely for a conception of rights, but rather proposing an alternative to Nozick's system that is defensible. ${ }^{87}$ A possible response to the claim that Nozick's natural rights are not sufficiently linked to the capacity for meaningfulness would be that the latter is only a general, and necessarily vague, underlying source of these rights, the substance of which is demonstrated by other means. But this is not an adequate response: if a human capacity is cited as the source and foundation of a conception of natural and universal rights, then it ought to imply that conception alone. Ultimately the universal aims of Anarchy, State, and Utopia are undermined by the contingency of this link. Even Kantian deontology itself has

${ }^{87} \mathrm{Ibid}, \mathrm{p} 152$. 
proved difficult as a foundation for a set of rights, as demonstrated by its reasonable but quite different expression in the theories of both Rawls and Nozick.

This illustrates one form of the more general problem facing liberal and moral theory: reaching some particular substantive result that meets the claim of universality. The general statements that underlie a theory may well lay claim to some form of universality, but the theory itself must be more narrow and particular, more closely specified, in order to reach a result that prescribes a particular set of rights, principles or rules to the exclusion of others. Once a theory adopts specific substantive ideals it is subject to the argument that these are not fully implied by its more general foundations. It may well amount to a reasonable expression of the latter, but it seems likely that it will not be the only one, and even the concept of reasonableness in this context is not beyond contest.

What is suggested here is a pervasive form of uncertainty, namely the contestability and openness of particular moral theories, which any universal liberal theory must overcome. This approach doubts there can be a well-defined theory that stands alone as the reasonable or best expression of any universal foundation. Rawls' theory, despite adopting a number of ingenious methods, does not overcome the assertion of intuitive bias in its structure and substance.

The criticism of Anarchy, State, and Utopia that persists is that its criterion of moral legitimacy, the system of strictly applied natural rights, is not adequately justified, in the sense of showing that these rights express their foundations to the exclusion of all alternative conceptions. It consequently fails to justify the infrangible nature of these rights. It would be expected that this argument would also be reflected in the criticism centred on the content and effect of Nozick's rights, that these rights are actually contestable and cannot carry the weight that Nozick assigns them.

As with much of Nozick's theory, the first criticism is one of inadequacy. His account of rights has been criticised both because it omits a theory of rights, which would explain their nature and operation, as well as a detailed exposition of the actual 
boundaries that they imply. ${ }^{88}$ Both of these arguments are valid: the lack of both an explanation of rights and an elucidation of the content of those rights is of some concern in a rights-based theory, but is not fatal to the most basic principles on which the theory is based.

Commentary has also focussed on what general statements Nozick does make about the nature and substance of these rights. Thomas Scanlon, for example, refers to Nozick's attempt at distinguishing between those interests that should be under our individual control, and those that should not. Nozick examines the idea that we have a right over those decisions that "importantly affect our lives." ${ }^{89}$ This he rejects on the grounds that it would give people an illegitimate level of control over the interests of others. He illustrates this with a number of examples of decisions that affect others. But, he suggests, that it seems wrong to give them the right to alter certain decisions: to accept or decline a proposal of marriage; as to how to use one's property; as to whether to retire; and, the decision of Thidwick, the big-hearted moose, to go across the lake against the wishes of the animals living in his antlers. ${ }^{90}$ The conception whereby these decisions can be coerced by others, Nozick argues, is clearly mistaken, and his conception of rights overcomes its shortcomings by considering how a decision affects our interests, and, in particular, whether it breaches our natural rights.

Scanlon objects to this on the grounds that it offers no clear and acceptable standard by which to allocate rights.

\begin{abstract}
I would agree that, while the importance of rights largely flows from the importance of having control over things that affect one, the function of a system of rights is to distinguish between the various ways that things can affect people and to apportion out particular forms of control. It follows that if we are agreed how this is to be done, then we will refer to people's rights in order to determine what they are entitled to cover. But it does not follow that Nozick's "Lockean" rights are the correct ones, and this is just what is at issue.... If a supposed right turns out to give the person holding it an obviously unacceptable degree of control over other people's lives then that is ground for saying that there is no such right. The proposed "right to have say over what affects you" fails this test. But ... unrestricted property rights of the sort favoured by Nozick must also be rejected on the same grounds. ${ }^{91}$
\end{abstract}

Nozick emphasises the importance of distinguishing between those individual interests and corresponding forms of control that are legitimately protected by rights,

\footnotetext{
${ }^{88}$ Nozick, op cit, above note 3, pp130, 32 .

${ }^{89}$ Ibid, p268.

${ }^{90}$ Ibid, pp268-269.

${ }^{91}$ T Scanlon, 'Rights, Liberty and Property' in Paul, op cit, above note 2, p117.
} 
and those that are not. But, as Scanlon notes, the basis of this discrimination is simply the system of Lockean rights; Nozick assumes without argument that his rights reflect the correct expression of this distinction. Scanlon goes on to suggest that Nozick's unrestricted natural rights can in fact lead to situations of illegitimate control, and, so, suffer from the same difficulty as having a right in relation to that which importantly affects our lives.

The example Scanlon offers is one that Nozick himself discusses, namely the legitimacy of pressuring an individual into entering into unfavourable conditions of employment by reducing the alternatives they have available. ${ }^{92}$ Nozick argues that if the other workers (and employer) all act voluntarily and within their rights, then this decision will itself be voluntary since no-one else has illegitimately restricted their alternatives. ${ }^{93}$ Scanlon asserts that this is an inadequate criterion, in that it does not sufficiently explain the illegitimacy of the situation, and also because the principle of voluntariness is difficult and would lead to disagreements in particular cases. ${ }^{94}$

The point Scanlon is making is that the simple reference to the Lockean rights, and some basic supplementary concepts such as voluntariness, is not sufficient. The rights must themselves be justified in the sense of showing why the control they offer over the interests of others is legitimate. He argues that Nozick fails to explain adequately the rule being applied here: that employers have the right to limit the alternatives of prospective workers, as long as they are not breaching any rights or removing the voluntariness of their decision.

\footnotetext{
The suggestion is that any interference with the rights of employers would be an intolerable intrusion - as a forcible reassignment of marriage partners would. Serious consideration of such a claim would bring Nozick's argument onto the same plane as the objection he is confronting. To settle the question between them, one would need an assessment of the relative importance of the various forms of liberty that are at stake and an account of how these and other values would be affected by alternative assignments of right. ${ }^{95}$
}

The illegitimacy of forcible marriage reassignment is an easy point to make, and, so, requires little additional explanation. But this is not true of the claim regarding the legitimate actions of employers. Nozick imports the concept of voluntariness in order

\footnotetext{
${ }^{92}$ Ibid, pp116-117.

${ }^{93}$ Nozick, op cit, above note 3, pp263-264.

${ }^{94}$ Scanlon, op cit, above note 91, p117.

${ }^{95}$ Ibid, p118.
} 
to flesh out the content of the relevant rights, but the doubt as to the role of this idea, and its effect in particular cases, makes the identification of particular rights difficult in a way that Nozick never really addresses or accepts. It is Scanlon's contention that this difficulty makes clear that the natural rights are not automatically correct in cleaving control appropriately. This means that, for Nozick's account of rights to be reasonably complete, he would need to actually identify and justify the content of these rights in terms of legitimate control.

The importance of this omission is demonstrated by Nozick himself, in rejecting the idea that we ought to have rights over all that importantly affects us; the uncertainty about which of these rights are legitimate, then, requires a complex substantive analysis. In the words of Scanlon, this would involve examination of the "relative importance of the various forms of liberty that are at stake" as well as the relevant values. ${ }^{96}$ But this manner of analysis is contrary to Nozick's general approach: assessing the relative importance of particular liberties implies a conception of rights that is contextual, and depends on the particular circumstances and values involved, rather than simply applying a set of natural, absolute rights.

Scanlon objects to his lack of justification in terms of the content of his rights, but, more significantly, to the assumptions that underlie his conception. Because Nozick's view is essentially libertarian, the rights he is espousing tend to be restricted, and would be agreeable to most liberal-minded people. It is easy not to question these basic rights. But Scanlon demonstrates the complexity and contestability involved in the justification of particular rights, and that Nozick's approach is inadequate in addressing this.

Another aspect of his conception of rights that has faced criticism is the principle of absolute non-interference: that the state cannot abridge to any degree the legitimate rights of individuals. The complexity as to the content of these rights does not sit easily with this principle, a point made by Nagel in identifying the different importance of different rights:

\footnotetext{
${ }^{96}$ Above, note 95.
} 
It is far less plausible to maintain that taking some of an innocent man's property is an impermissible means for the prevention of serious evil, than to maintain that killing him is impermissible. These rights vary in importance and some are not absolute even in the state of nature... The sources of morality are not simple but multiple; therefore its development in political theory will reflect this multiplicity.... The only way to make progress in understanding the nature of individual rights is to investigate their sources and their relations to each other and to the values on whose pursuit they set limits. ${ }^{97}$

The assertion that different rights carry different levels of importance is entirely plausible, and brings into question the strict application of Nozick's conception of rights. At the most basic level, Nagel argues that the application of rights should reflect the variable importance of the interests they protect. He goes on to suggest that the plural nature of morality underlies this variation in importance, and means that there may be circumstances where rights should be sacrificed to some ends, since these ends can reflect morally important interests. ${ }^{98}$ The conclusion Nagel draws is similar to that of Scanlon: that dealing fully with these rights requires a careful analysis; for Nagel, this involves the sources of the rights and their relationships with other rights and welfare-effects.

Nozick seems to make some overly simplistic assumptions about the nature of morality, and the rights a theory of justice should protect. In the words of Bernard Williams, "his conception of justice does look like an enormous exaggeration of at best one aspect of our moral ideas." $" 99$ To demonstrate this point Williams offers the following example:

[S] uppose that, when the Mayflower arrived, some foresighted fellow, crouching by the gangplank, jumped off and bagged a good area of what is now Massachusetts, before his companions, more cooperative, pious, idle, or enfeebled, got going; it looks as though Mr Nozick, if we assume there were no prior holders, would grant him just title. Do we agree? Would it be unjust to redistribute in favour of those others (even the idle)?... These are questions for Mr Nozick's theory of justice ... but we can notice more broadly that, even if we eventually agreed that this pushy settler was not to be faulted in justice, that would only underline the point that we could hope that the Pilgrims, and ourselves, would have arrived with more virtues than justice. We are reminded again of that richer range of moral resources... which Mr Nozick's treatment systematically leaves out. ${ }^{100}$

The general point that these arguments raise is that Nozick relies on a set of rights that reflect a particular, contestable view of justice and that are bereft of substantive complexity. They ignore variations in the nature and importance of the interests they

\footnotetext{
${ }^{97}$ Nagel, op cit, above note 2, p196.

${ }^{98}$ Ibid.

${ }^{99}$ B Williams, 'The Minimal State' in Paul, op cit, above note 2, p34.

${ }^{100}$ Ibid, pp34-35.
} 
protect, the relationships between rights, and other matters relevant to morality and justice. The approach of applying the rights absolutely as the sole moral criterion itself appears overly simplistic and morally illegitimate.

\subsection{The ultraminimal state}

The doubt as to the correctness of Nozick's natural rights will undermine the remainder of his theory. Despite this, it is useful to examine its more substantive aspects. As well as including an analysis of his reasoning separate from the rights justification, this will allow a discussion of Nozick's criticism of patterned and endstate distributive principles.

The first step in Nozick's argument is to derive the ultraminimal state from the state of nature. ${ }^{101} \mathrm{He}$ achieves this by imagining a hypothetical situation where protective agencies are employed by individuals, and which results in a single, dominant protective agency that is employed by virtually every individual. This occurs without any rights being breached, and has the additional parsimony of an invisible hand explanation. In this way Nozick shows how a state, or something resembling a state, can arise legitimately out of a state of nature without the use of coercion or the adoption of illegitimate powers.

As with Rawls' original position, Nozick employs a hypothetical narrative in his theory. The two are used in different ways: the original position is supposed to reflect the principles of justice that rational individuals would adopt given particular limitations on their knowledge. This situation is supposed to be recognisable as a morally valuable reflection of our actual circumstances. But such recognition cannot play the same role in Nozick's theory, since his focus is on a conception of rights that is true rather than intuitively acceptable. The moral significance of Rawls' result comes from the general acceptance of the original position and the two principles as a reflective equilibrium; the significance of Nozick's ultraminimal state is less obvious.

${ }^{101}$ Nozick, op cit, above note 3, pp7-8. 
Williams questions the moral value of the ultraminimal state: "What weight is there in the fact that we could, relative to certain wildly idealized psychological assumptions, reach the state without violating anyone's rights?"102 Nozick's hypothetical narrative shows how a government could arise without any morally impermissible steps. This is important since his admitted starting point for political philosophy is to justify the state relative to a state of nature. ${ }^{103}$ By showing that a government could arise given the legitimacy constraints, Nozick defeats the anarchist argument that governments are necessarily wrong by virtue of their coercion of the individual.

But Nozick's conception of the ultraminimal state has a greater justificatory role than merely showing how a legitimate state could arise.

A theory of a state of nature that begins with fundamental general descriptions of morally permissible and impermissible actions, $\ldots$ and goes on to describe how a state would arise from that state of nature will serve our explanatory purposes, even if no actual state ever arose that way. ${ }^{104}$

Nozick talks not of how a state could arise from his state of nature, but of how it would arise, given the legitimacy constraints. He envisages that, if these constraints were applied, the ultraminimal state would be the natural outcome without any conscious direction given the incentives of the individuals and associations involved. The justificatory power of the ultraminimal state, then, is that it is the plausible and likely outcome of a state of nature where every person and group acted according to settled moral rules. This power subsists regardless of whether any state actually arose in this way, and, in fact, it would be unlikely that any state could ever actually develop legitimately. ${ }^{105}$

Contrary to Williams' comments, this result seems to be able to bear much weight. If the legitimacy constraints were settled then Nozick's account of the development of the ultraminimal state from the state of nature is compelling, as is the conclusion that this state is a plausible theoretical foundation for a legitimate government. These constraints have been convincingly discredited, but, if this was the only problem with Nozick's approach, it might be possible to replace them with an agreed set of

\footnotetext{
${ }^{102}$ Williams, op cit, above note 93, p33.

${ }^{103}$ Nozick, op cit, above note 3, p4.

${ }^{104}$ Ibid, p7.

105 Ibid.
} 
constraints, and then look to the state that would arise from the corresponding state of nature. However, there are doubts not only as to the possibility of agreement on such constraints, but also as to the reasoning from state of nature to ultraminimal state.

Nozick approaches political philosophy from the perspective of what he argues to be its main alternative, anarchism. The state of nature reflects this perspective; the hypothetical and legitimate moves from it represent acceptable improvements on anarchism. The process of reaching a legitimate state is simply a bundling of individual rights, vested voluntarily in a central association. But this is not the only possible theoretical perspective. Instead of viewing the legitimate state as a response to anarchism, and, so, one that grows theoretically out of a state of nature, one might instead, see the state as a necessary response to a complex society of individuals. In this case, the theoretical focus is on, not the state as restricted to legitimately obtained rights, but the state in its capacity to achieve certain social goals: to co-ordinate action; to maximise some measure of the goodness of the lives of its members; or, to further their opportunities or the exercise of their essential capacities. If the focus is on some conception of justice not based so strongly on natural pre-political rights, then the state will be developed from its capacity to carry out an alternative conception of justice.

The point is that Nozick's initial theoretical perspective, that of anarchism or legitimate moves from a state of nature, seems plausible, but not so as to refute other possible approaches. ${ }^{106}$ A universal approach would involve identifying a particular theory as being the best perspective and would, in turn, require locating it within a general conception of moral theory. Nozick does discuss his theory in relation to endstate and patterned principles, which can be taken as the first steps to towards a general view of such moral theories; and, it is in this argument that his refutation of other perspectives of justice must lie.

\subsection{The minimal state}

\footnotetext{
${ }^{106}$ Nagel in Paul, op cit, above note 2, p194.
} 
The shift from the ultraminimal to the minimal state introduces new functional concepts, including prohibition and compensation, which are in themselves questionable, and the effect of which seems contrary to the natural rights framework that underlies all of Anarchy, State, and Utopia. The new tools seem to reflect the difficulties in applying a strict rights framework to an increasingly complex situation. According to a simple rights analysis, actions that breach the rights of others should not to be permitted unless the victim freely consents to the breach. Conversely, any action that does not breach the rights of others ought to be permitted.

This basic approach fails to deal adequately with two significant situations. The first is where prior consent would be very costly or impossible to gain. In this situation, Nozick argues, the act could be permitted if accompanied by appropriate compensation. ${ }^{107}$ The second and more significant situation is where there is some chance that an act will breach the rights of another. This risky behaviour can either be permitted, and compensation paid to the victim for actual or expected breaches, or prohibited, and the person who is restricted compensated or not. ${ }^{108}$

In one sense, dealing with these situations outside the basic rights analysis appears to be reasonable and deepens that analysis. But in another, it does not sit easily with the simple picture of strict rights set out in earlier parts of the theory. To determine whether a particular risky action is to be prohibited, for example, and what form of compensation should be used, requires a complex contextual analysis of the circumstances involved; with this deeper analysis the emphasis seems to shift from whether a right is breached to the nature and effect of a possible breach. This may reflect the true-to-life complexity of a rights-based system, but it seems contrary to the essence of Nozick's legitimacy criteria.

This concern is present in the criticism of the shift from the ultraminimal to the minimal state, which comments generally on the technicality and difficulty of the required reasoning. Perhaps the most cogent summary of this criticism is presented by

\footnotetext{
${ }^{107}$ Nozick, op cit, above note 3, p72.

${ }^{108}$ Ibid, p81.
} 
Keith Hyams, in 'Nozick's Real Argument for the Minimal State. ${ }^{109}$ He argues persuasively that in order to deal with risky action, Nozick invents what he labels the 'compensation for disadvantage paradigm,' which "applies when, and only when, one agent enforces a morally sanctioned prohibition on another agent's risky activities."110 In this situation the person whose action is prohibited is entitled to compensation when they have been disadvantaged by the prohibition relative to the normal situation, and not for what the person would have been, or could have been, if permitted to act.

This important principle is supposed to avoid the situation where someone has to compensate the person whom he or she prohibits from forcing them to play Russian roulette. ${ }^{111}$ This approach, however, suffers from many of the suggested problems: the implied standard of a 'normal situation' against which Russian roulette is judged is a difficult one, and is described and defined by nothing more than the exclusion of an obviously abhorrent game; and, this idea seems to be used only to make compensation with prohibition work, rather than as a necessary and justified part of a broader rights framework. Even in this most obvious of cases it seems that the conception used by Nozick, of disadvantage relative to a normal baseline, is one informed by our intuitive judgment of what is legitimate and illegitimate coercion rather than according to the conception of natural rights.

Even if the rules for risky action are inconsistent with the basic natural rights approach, there is no denying the elegance of Nozick's next move: identifying the action taken by protective associations (or individuals) in investigating and punishing rights infringements as a form of risky behaviour. In this way the rules on risky behaviour are applied to protective associations. The outcome of this is that the actions of small associations can be prohibited by the dominant protective association, which will itself undertake to protect those affected by this prohibition. The result is that the dominant protective association enjoys a de facto monopoly on settling rights disputes, and, in this capacity, can be fully recognised as an effective state. ${ }^{112}$

\footnotetext{
${ }^{109}$ K Hyams, 'Nozick's Real Argument for the Minimal State' (2004) 12(3) The Journal of Political Philosophy 353, p354.

${ }^{110}$ Ibid, p359.

${ }^{111}$ Ibid, p79.

112 Ibid, p109.
} 
This move appears to contradict the idea that individuals have the right to prohibit or punish violations, and that they cannot be coerced into forgoing this right in favour of another party. To overcome this, Nozick applies the risky-action reasoning: procedures for prohibiting or punishing rights violations are not themselves perfect, but depend on the person being guilty of the violation, or not being entitled to commit the act; these procedures, as risky actions, can themselves be prohibited if the person or association is not in the best feasible position for ascertaining this violation. ${ }^{113}$ In this way the dominant protective agency can gain legitimately the power to prohibit the procedures of others, since they are in the best position to judge the situation.

This move has been criticised by Robert Holmes, who objects that it implies the dominant protective agency itself uses procedures of proven reliability and fairness.

\begin{abstract}
Yet there is no independent criterion by which to establish this to be found in Nozick. He simply reiterates that the DPA will apply its own criteria according to its lights and will be the one entity in the position to enforce their acceptance. If reliability and fairness are defined in terms of what the DPA decides, this of course is question-begging and yields a theory of "might makes right." 114
\end{abstract}

If there is no reason to think the dominant protective agency's procedures are going to be any better than those of any other association, then Holmes' criticism is merited. There are two complementary arguments against this view. The first is that the development of a non-arbitrary decision procedure is one of the determinants of the success of a protective association, so that it might be expected that any dominant association has a well-developed non-arbitrary procedure. ${ }^{115}$ Secondly, the power to prohibit is founded not on the association's position of dominance, but on being in the best feasible position to judge rights disputes. These suggest that the dominant association's procedures will tend to be fairer and more accurate than those of any other association. This idea blunts the might-makes-right accusation of Holmes. Arguments about the reasoning from the ultraminimal to the minimal state seem better directed at the underlying legitimacy assumptions or the technical rules used, rather than at disputing what goes on in the hypothetical world.

\footnotetext{
${ }^{113}$ Ibid, pp106-107.

${ }^{114}$ R L Holmes, 'Nozick on Anarchism' in Paul, op cit, above note 2, pp63-64.

${ }^{115}$ Nozick, op cit, above note 3, p13.
} 
The second argument Nozick makes in favour of the minimal state is that it has utopian value, not in that it champions one conception of how to live, but as a framework that permits people and groups to choose their own version of utopia, unmolested by the state. ${ }^{116}$ Smaller communities can form that adopt some common understanding and rules that apply to all members, but there is always the right to exit this community and live elsewhere. ${ }^{117}$ Barbara Fried has criticised this argument as being inconsistent with the remainder of Nozick's theory:

\begin{abstract}
Why does it not follow from the justificatory role of exit at the community level that any state, with any compulsory package of rights/restrictions/prohibitions ... is morally justified, as long as dissenters who do not like the particular package are permitted to exit? And why then didn't Nozick limit his ambitions in [the other parts of his theory] merely to proposing his libertarian version of utopia to compete, in the marketplace of state-ideas, with all those mildly redistributive, social-welfarist, democratic states out there, in the hope that he could drum up enough takers to get it up and running on some utopian island somewhere ${ }^{118}$
\end{abstract}

There does seem to be a thematic conflict between the idea, central in Nozick's theory, that compelling another to act in a certain way is illegitimate, and the idea that communities can legitimately set rules that members must follow, as long as they are free to exit. Fried argues that these two threads cannot be adequately reconciled. This view is most obviously illustrated by Nozick, arguing, on one hand, that no state can legitimately go beyond the minimal, and, on the other, that the right of exit justifies any manner of super-minimal compulsion.

Nozick's response to this criticism is to distinguish between nations and communities, so that his libertarian analysis applies to and justifies the central government; whereas communal compulsion is permitted only at a sub-state level, to communities that are embedded in the libertarian framework and are justified by the consent of their members and have the right of exit. ${ }^{119}$ However, as Fried recognises, the distinction between nation and community is not coherent, ultimately because Nozick relies on a conception of harm by offence, whereby people can be harmed by the non-conformity of others within a community, but not within a nation. ${ }^{120}$ This leads even Nozick to question what powers of compulsion communities and nations can adopt in relation to harm caused by offence, and to state that he does not see his way clearly through the

\footnotetext{
${ }^{116}$ Nozick, op cit, above note 3, p312.

${ }^{117}$ Ibid, p321.

${ }^{118}$ Fried, op cit, above note 1, p224.

${ }^{119}$ Nozick, op cit, above note 3, p320.

${ }^{120}$ Fried, op cit, above note 1, p 225.
} 
issues involved. ${ }^{121}$ Ultimately, then, this idea of utopian value, and its place within the boarder theory and rights framework, is not adequately dealt with by Nozick. It is interesting to note, however, that his attempts to set up a framework, a meta-system of rights within which people or groups can pursue their own conception of the good, is similar to that of Rawls, and much of contemporary liberal theory. It is also interesting to find Nozick, in considering how communities can make rules that compel their members, grappling with majoritarianism, despite promoting a theory based on consent and strict rights. ${ }^{122}$

\subsection{Beyond the minimal state}

The claims made by Nozick in arguing against any state beyond the minimal are of fundamental importance, since they involve a general criticism of any distributional principle of justice characterised as end-state or patterned.

Certainly Nozick's distinction does capture something intuitively appealing. It has often been said as a criticism of utilitarianism that it ignores morally significant relations resulting from past actions. Nozick's distinction is of great interest if it gives this criticism a more abstract form and shows it to apply not only to utilitarianism but also to Rawls' theory and to virtually every other commonly offered. ${ }^{123}$

The basis of this criticism is that other such theories cannot accommodate the dynamic distributional effect of transactional freedom, an effect which over time can only distort the end-states or patterns that were originally the primary focus of the principles. Such a theory must either allow this distortion, or curtail an intuitively valuable freedom. This is clear from the Wilt Chamberlain example: either Wilt is allowed his money and the original equality is seriously undermined, or people are prevented from entering into an entirely voluntary and mutually beneficial agreement with him. As Scanlon notes above, this idea has the potential to pervade much of distributional moral theory.

Scanlon, however, ultimately rejects the power of this idea on the grounds that he doubts whether anyone actually holds a patterned conception of justice:

\footnotetext{
${ }^{121}$ Nozick, op cit, above note 3, p323.

122 Ibid.

${ }^{123}$ Scanlon in Paul, op cit, above note 91, pp109-110.
} 
A person who objects to the inequality in the world is unlikely to be concerned with those who have less as a result of their giving away or trading part of what was once an equal share. What offends an egalitarian primarily is the great inequality in the initial resources people have as a result of the social positions in to which they are born. ${ }^{124}$

Scanlon notes that the right to retain any resources that others are willing to pay for is, like egalitarian patterns of distribution, not necessarily absolute. ${ }^{125} \mathrm{He}$ goes on to describe a number of viable theories that are not patterned, but are still, broadly speaking, egalitarian. ${ }^{126}$

The possibility of theories that are mixed, in that they have an egalitarian focus yet allow some degree of transactional freedom, is a common, and plausible, response to Nozick's claims. ${ }^{127}$ Rawls' theory, the main object of Nozick's criticism, itself probably falls within this mixed category. It prescribes equality in liberties, including primary goods, but allows inequalities that favour the worst off, and which would cover many consensual transactions. In this way it offers a basic criterion for permissible transactions and is partially historical in the sense that it focuses on the original distribution and the consequences of a transaction in order to determine its legitimacy. $^{128}$

Nozick does succeed in identifying a weak part of Rawls' theory, by emphasising that he does not adequately deal with consensual transactions, and particularly their cumulative effect on a distribution. But it is to be expected that, since property rights, including transactional rights, are central to Nozick's theory, his will be more transactionally dynamic than a theory where the primary concern is that of distributional fairness.

Nozick's distinction between historical, and patterned or end-state theories, then, is important, in that it shows that the desired effect of a principle of justice on the distribution of resources can be undermined by the freedom to use and transact with these resources, and that this would increase over time. But, the effect of this on other

\footnotetext{
${ }^{124}$ Scanlon, op cit, above note 91, p110; A Kaufman, 'The myth of the patterned principle: Rawls, Nozick and entitlements' (2004) 36(4) Polity 559, p563.

${ }^{125}$ Scanlon, op cit, above note 91, p111.

${ }^{126}$ Ibid, pp111-113.

${ }^{127}$ E.g. Nagel in Paul, op cit, above note 2, p201.

${ }^{128}$ Kaufman, op cit, above note 106, p563.
} 
theories is overplayed by Nozick, primarily because he views any theory that does not actively promote the transactional freedoms as being ultimately destroyed by them. In this way, he fails to recognise that there can be more complex relationships between distributive justice and transactional freedom than the simple priority of one over the other. Ultimately, A Theory of Justice needs to evince an effective interplay between the two, such that, the nature of valid resource transactions are defined, and that this is related to the distributive goals or processes in such a way that neither is overwhelmed by the other. With a theory that has an emphasis on some form of egalitarianism or resource equality, this would involve dealing with matters such as fair opportunity and education, dessert, gains from trade, illegitimate and coercive bargains, intergenerational wealth and non-wealth resources. Rawls' theory discusses many of these in a complex and enlightening manner, yet he never deals with the full extent of the problem identified by Nozick.

\section{Conclusion}

Although the political prescriptions of Rawls and Nozick are diametrically opposed, there are many similarities of approach and structure between the two. Generally, both attempt to build theoretical, universally justified structures. Nozick's method is probably more primitive than that of Rawls, in that it is based on a supposed universal standard of natural rights, independent of the rest of his theory. The biggest questions of Anarchy, State, and Utopia are those that challenge the justification and substance of these rights as the primary criterion for morally legitimate actions.

Rawls' approach makes a more sophisticated use of our intuitions and considered judgements, as well as the judgments of those hypothetical people in the original position. Yet these depend on another supposedly universal standard, namely the priority of the right over the good. Despite the additional complexity and elegance of Rawls' approach, this priority is an absolute in much the same way as Nozick's natural rights. Both are deontological expressions that reflect the Kantian ideal of a person as an end rather than a means of value; so, with Rawls, matters of the right will not be compromised by a society's total welfare, and, with Nozick, natural rights will 
not be compromised by the welfare of others. In the context of liberal theory, both define a meta-system of rights with a substantial liberal space, within which individuals can pursue their own conceptions of the good, or of utopia.

But these two priorities suffer from the same basic problems: they fail to adequately justify their respective rights conceptions as being absolutely morally prior to other possible approaches or beliefs. Both benefit from some strong intuitive plausibility, yet in neither case is this adequately confirmed as a universal priority. These failures are essential to the respective theories. For Nozick, the absolute value of his natural rights is the central universal foundation supporting his theory. Rawls' position is more complex: for him, the priority of rights over welfare is not simply declared at the beginning, but is agreed to both in the original position, and in reflective equilibrium, and reflects a broader conception of the right as being moral and common, and the good as individual and base. Without these theoretical struts, neither theory can stand against the arguments of moral uncertainty.

These failures seem to occur much in the same way: some absolute and seemingly reasonable conception is drawn that, upon investigation, is found to be not rationally supportable; the distinctions involved are drawn too sharply and aren't actually valid; complexities are ignored; and possible alternatives are rejected without consideration. Both theorists seem to make the error of apotheosising a particular moral intuition, or group of intuitions. With Nozick it is that we should control both those matters that are within our personal spheres, and those which we have created or earned. For Rawls, justice is based on fairness within a society, which emphasises the importance of a fair equality between people. What neither Rawls nor Nozick seem to appreciate is the contestability of such intuitions, which corresponds with the uncertainty in reaching some morally universal result. Attractive as these ideas are, neither has been shown to be the valid foundation for a comprehensive and universally just political system.

The mere fact that both these theories exist and have been influential in political theory raises doubts as to the universal value of either, or, at least, as to the ability to demonstrate them as universal and our ability to recognise this. These two divergent moral and political positions were reached by two rational, reasonable and sincere 
people educated in the same tradition, using similar theoretical tools. That two such people can differ so markedly in the content of their deontological priorities, and, so, in their theories and results, raises serious doubts as to the potential effectiveness of such political theories.

To overcome these doubts would require either some different approach to justifying such priorities or an approach to moral uncertainty that does not involve any such priority at all. It is conceivable that comparable results, results universal in some importance sense, might be reached without recourse to a definite and universal moral standard. Perhaps the desire to vanquish what was the dominant political paradigm prior to Rawls, utilitarianism, caused both theorists to err on the side of deontology, and, so, to set up a standard of absolute priority that allows no semblance of trading off with welfare. Their motives in doing so are expressed well by McNaughton in his criticism of Rawls' distinction between the right and the good:

Can the notions of the right and the good ever be made congruent, or are they simply mutually exclusive? It appears that once the element of right is allowed into an ethical theory, it must, by definition, take precedence over the good. If right does not take precedence over the good, then right necessarily becomes relative to the good, thus becoming a good itself. That is, if right is relative, and not "absolute," its value must then be relative to some perceived good. ${ }^{129}$

McNaughton expresses the concern that anything less than an absolute priority might leave Rawls and Nozick open to the utilitarian argument that such rights reflect and express the welfare of individuals, and are valuable in this respect, or, in other words, that their theories are really just expressions of utilitarianism in disguise. But, such an argument need not succeed. It is, in fact, quite possible to attach some value to a moral standard or a corresponding set of rights that is not absolute in nature and, yet, is not reducible to utilitarianism. There can be complex relationships between individual welfare and other moral issues that mean neither an absolute priority nor a simple trading off, as has been recognised and suggested in relation to Nozick's natural rights. ${ }^{130}$ Such a position would better reflect the complexity of recognising and delineating the moral and the right, and could, conceivably, better reflect the contestability of moral values and, so, represent a more effective response to moral uncertainty. But, a theory based on such rights would face a whole new set of

\footnotetext{
${ }^{129}$ J McNaughton, 'John Rawls and the Conflict Between Right and Good' (1980) Auslegung 270, p277.

${ }^{130}$ Scanlon, op cit, above note 91, p128; Scheffler, op cit, above note 84, p152.
} 
challenges: whether and how universality might be achieved while admitting contestability, or at least complexity, of morals.

A Theory of Justice and Anarchy, State, and Utopia both employ a deontological priority as part of a theoretical structure that describes and justifies a distinct set of rights: equality of liberty in one, and a system of natural rights in the other. Despite their theoretical ingenuity and elegance, and notwithstanding their substantive inconsistencies, both fail as responses to moral uncertainty, as the priorities of both are conceived in a way that ignores the complexity and contestability of our moral lives. Both confuse strong intuitive plausibility with moral universality and, but for an extended explanation of their priorities or a new approach to justification, the projects of both must fall short on those terms. 


\section{EQUALITY AND NEUTRALITY}

\section{Introduction}

In the years following Anarchy, State, and Utopia, up until the early 1980s, liberal theory focussed on more egalitarian and recognisably Rawlsian conceptions. This chapter will focus on the two significant expressions of liberal theory that largely precede the criticisms and alternatives set out by those critics, collectively known as the communitarians, who will be examined in the next chapter.

The theories of Ronald Dworkin and Bruce Ackerman are undeniably post-Rawlsian, in the sense that they adopt some of the assumptions and arguments from A Theory of Justice. But, they represent developments in the theory and respond to some of those criticisms of Rawls' theory discussed in the earlier chapter. The goal of exploring their work is to identify and test these developed justifications and approaches against the criticisms levelled at Rawls and Nozick, and against moral uncertainty generally. This will involve looking at any absolute priorities or standards and inquiring as to whether they have been plausibly justified against other considerations, and looking at the resulting relationship between the liberties defined by the theories and other concerns such as welfare.

The primary difference between Dworkin's and Ackerman's work, and that of Rawls, is the shift in the concept used as the central liberal focus. If $A$ Theory of Justice were to be reduced to one substantive and driving concept, it would be justice as fairness; the fairness that underlies the details of the original position and from which the principles of justice are drawn. With Dworkin and Ackerman, the primary focus is more on equality and neutrality respectively.

\section{Dworkin and equality}




\subsection{Equal concern and respect}

In his book Taking Rights Seriously (1977), Dworkin identifies the somewhat hidden but central and basic right of Rawls' theory: equal concern and respect. It is this right, he argues, that implicitly informs the design of the original position and the setting aside of the natural and social differences between individuals as being morally arbitrary. ${ }^{1}$ It is this right, then, from which the two principles of justice stem.

We may therefore say that justice as fairness rests on the assumption of a natural right of all men and women to equality of concern and respect, a right they possess not by virtue of birth or characteristic or merit or excellence but simply as human beings with the capacity to make plans and give justice. ${ }^{2}$

In effect, Rawls' fairness is an expression of the basic and abstract right to equal concern and respect, and the original position is the means by which more concrete rights are derived from it.

Dworkin's view of liberalism, then, amounts to a conception of equality out of which various liberties can be drawn. In his view, this amounts merely to a clarification of Rawls' approach, a bringing into focus the equality that is already its driving force. But, by making this point explicitly, Dworkin is able look directly to equality to determine the best set of liberal rights. This equality, he argues, requires that individuals be treated as equals, in the sense that each has the right to equal concern and respect in political decisions about how goods and opportunities, broadly understood, are to be distributed. ${ }^{3}$ This does not necessarily amount to a strict resource equality or egalitarianism, but, rather, means that, in the process of determining the desired distribution, each person's interests will be treated equally.

There seems to be three closely linked consequences of such an approach. The first is the protection of some of the basic political liberties; in order to be treated equally, individuals need the right to free speech and to vote. The second consequence is that no person's conception of the good should be applied to society generally, since this

\footnotetext{
${ }^{1}$ R M Dworkin, Taking Rights Seriously (1977), p181.

${ }^{2}$ Ibid, p182.

${ }^{3}$ Ibid, p273.
} 
would treat those who hold that conception as more important than those who do not. ${ }^{4}$ The final effect relates to decisions based on utilitarian reasoning. Superficially, utilitarianism seems to satisfy equal concern and respect in that everyone's interests is taken into account and weighted equally. The problem with utilitarianism, Dworkin argues, is that it incorporates external preferences, the preferences we have that are not about our own situation, but relate to the assignment of goods or opportunities to others. ${ }^{5}$ Examples of such preferences are racism, and the disapproval of the actions of others in relation to homosexual practices or the use of contraception. ${ }^{6}$ These preferences ought not to be counted as they affect the way that the individuals in question are treated politically, and mean that the concerns of others are prior to their own.

So, utilitarianism, and for that matter democratic majoritarianism, is not capable of fully expressing an equal concern and respect for every individual. Rights are necessary to overcome the defects in these ideals, and must, in order to preserve equal concern and respect, overrule or trump their operation. ${ }^{7}$

Individual rights are political trumps held by individuals. Individuals have rights when, for some reason, a collective goal is not a sufficient justification for denying them what they wish, as individuals, to have or to do, or not a sufficient justification for imposing some loss or injury upon them. ${ }^{8}$

This 'rights as trumps' approach may be drawn from what Dworkin believes to be the underlying concern of $A$ Theory, but it differs in approach and effect. Although only sketched briefly by Dworkin in Taking Rights Seriously, these differences can support some comment.

The ideal of equal treatment and concern in political decisions is certainly present in the conditions of the original position, yet this is only one aspect of Rawls' justificatory process. In effect, Dworkin has extracted from Rawls' theory one important underlying conception, and built around it a simpler structure that justifies rights and distributive policies. The idea of deriving liberties from this equality that correct for the defects of utilitarianism and democracy is plausible. The nature of

\footnotetext{
${ }^{4}$ Ibid, p274.

${ }^{5}$ Ibid, p275.

${ }^{6}$ Ibid.

${ }^{7}$ Ibid, pp266-267.

${ }^{8}$ Ibid, pxi.
} 
these defects is that the latter incorporate external preferences; individual conceptions of the assignment of resources to others. Effectively, he is arguing that being treated as equals in the decision of how to distribute resources prohibits taking account of what people think others should have, and includes only preferences as to our own interests.

It is clear that utilitarianism and democracy include such considerations, in that they are simple expressions of preference. What is unclear is whether Dworkin's conception of being treated equally actually excludes these, and whether they should to be excluded anyway. Dworkin argues that the expression of external preferences in a political decision implies unequal treatment, but this seems to confuse the outcome of a decision with the decision-making process. If racist preferences, for example, are taken into account in determining resource allocation, this might mean that one race receives a lower level of resources and opportunities than they might otherwise have received. This does not mean that they were treated unequally in the decision-making process itself. Taking account of external preferences may lead to resource inequality, but this is quite different from being treated unequally. Dworkin himself notes that a person's interest may be taken into account and yet outweighed by the interests of others, "and in this case their right to equal concern and respect, so defined, would provide no objection". ${ }^{9}$ This being the case, Dworkin offers no reason, in terms of equal treatment, why external preferences should not be taken into account.

It seems unusual that Dworkin wants to exclude external preferences anyway. In his argument this appears as the removal of discriminatory beliefs - illegitimate and ugly ways that people want to interfere in the lives of others. In fact, external preferences include all moral judgements and beliefs about distributive justice. His own predilections for equal treatment and their distributive consequences could be accurately described as an external preference, and his system is its embodiment. If his system is to express this external preference while excluding all others, Dworkin needs to give a better explanation of why this is.

\footnotetext{
${ }^{9}$ Ibid, p273.
} 
The final criticism of Dworkin's conception of liberal justice from Taking Rights Seriously must be levelled against the idea of equal concern and respect itself. This concept is the primary focus of his system, and is treated by him as the primary concern of justice, one that overrules the effect of utilitarian and democratic ideals. It is this superiority that justifies the priority of the rights that define his system's liberal space; in this sense, it performs the equivalent role of Rawls' priority of the right, or Nozick's natural rights. Dworkin's conception of equality is strongly attractive, especially in the abstract form in which it is presented. But Dworkin makes no realistic attempt to justify it as the primary or dominant principle of justice. He identifies it as the operative principle of $A$ Theory, and argues for its value in relation to utilitarian or majoritarian approaches, but neither of these adequately explains why equal concern and respect should be the primary consideration of justice, as opposed to a system of natural rights or some other conception of equality. Perhaps this can be excused on the grounds that Taking Rights Seriously was not supposed to be an expression of a coherent liberal position in the same way as A Theory of Justice or Anarchy, State, and Utopia. Yet, it contained a degree of support and confidence in his conception of equality that was not sufficiently justified by his arguments, and that contributes to an understanding of his later work on equality.

\subsection{Distributive equality}

In a number of subsequent essays Dworkin developed and extended his project of liberal equality. In the introduction to the recent collection of these he labels equal concern as "the sovereign virtue of political community", without which government is tyranny. ${ }^{10}$ This reflects his continued belief in his conception of equality as the central and primary subject of liberal theory, from which other matters, such as rights and distributive outcomes, are derived.

In 'Liberalism' (1978), Dworkin attempts to refine further his conception of equality, and to identify the source of its value. He asserts that the constitutive, meaning the essential and intrinsic, part of liberal theory is expressed by equal treatment, in the

\footnotetext{
${ }^{10}$ R M Dworkin, Sovereign Virtue: The Theory and Practice of Equality (2000), p1.
} 
sense that decisions are independent of particular conceptions of the good life. ${ }^{11}$ This account of equality, he argues, best satisfies the conditions of a constitutive moral theory of liberalism: it represents authentic, commonly held liberal intuitions; it is sufficiently complete to constitute a liberal core; it is sufficiently detailed to distinguish it from other liberal conceptions; and, it is as abstract and general as the other conditions allow. ${ }^{12}$

In two subsequent essays, Dworkin attempts to find the best expression of this distributive equality. It is this exploration of the moral and liberal meaning of equality that represents Dworkin's most significant and most novel contribution to recent liberal theory.

Equality is a popular but mysterious political ideal. People can become equal... in one way with the consequence that they become unequal ... in others. If people have equal income, for example, they will almost certainly differ in the amount of satisfaction they find in their lives. It does not follow, of course, that equality is worthless as an ideal. But it is necessary to state, more exactly than is commonly done, what form of equality is finally important. ${ }^{13}$

In order to do this he considers two types of approaches to distributive equality: those that promote an equality of welfare, and those that promote an equality of resources. The former involve taking the differences between people into account in order to equalise as much as possible the welfare of each, however this is measured, whereas the latter are insensitive to such differences, and entail an even division of the resources available.

Dworkin's first task is to survey a number of potential approaches to applying the equality of welfare standard, corresponding with several different measures of welfare, and to reject this standard generally. Welfare, he argues, seems like the best approach since it means equalising the well-being of each person, rather than their actual wealth. ${ }^{14}$ This welfare could be measured in a number of ways, the first of which Dworkin identifies as success measures of welfare. These focus on matters such as fulfilling preferences, goals and ambitions. He separates preferences into political (preferences about how resources and opportunities ought to be distributed),

\footnotetext{
${ }^{11}$ R M Dworkin, 'Liberalism' in S Hampshire (ed.) Public and Private Morality (1978), p127.

${ }^{12}$ Ibid, pp121, 128.

${ }^{13}$ Dworkin, op cit, above note 10, p11.

${ }^{14}$ Ibid, p14.
} 
personal and impersonal (which correspond with his earlier distinction between internal and external preferences). ${ }^{15}$

Dworkin's argument as to why these measures of equality fail centres on showing how each of these three types of preferences are not in themselves relevant to distributive justice. If political preferences were included, then individuals who had preferences that were not taken into account in the distribution (racists for example) would have to be compensated for this. ${ }^{16}$ To reward such political disappointment in the name of equalising success is clearly problematic, and in this sort of example, quite grotesque. Equally, impersonal preferences should not to be included in the equality calculation as this would mean incorporating such things as the hope that there be life found on Mars, or that the Great American Novel be written in a person's lifetime. ${ }^{17}$ It seems clear that these are also invalid as part of a just resource distribution.

That leaves personal preferences. Here Dworkin moves away from his earlier idea of incorporating personal preferences as a valid part of resource distribution, and instead argues that these would also distort any equalising process. This argument is expressed in a number of distinct ways, which are interesting in themselves, and which form the basis of his conception of distributive equality. One of the initial arguments is that personal preferences fail to take account of important parts of our lives by excluding the external and the impersonal. ${ }^{18}$ Even if preferences are not a valid means of including these in an equalising process, it does not mean that these areas ought to be excluded entirely. In other words, an equality of welfare that is nothing more than the equality of personal preferences is morally impoverished.

Dworkin goes on to explore what such equality might actually entail. It might imply, for example, an equal chance of success in meeting one's own goals. But this cannot make sense, since the person who chooses goals well within their abilities would receive few resources relative to the person who chooses impossible goals. ${ }^{19}$ Perhaps,

\footnotetext{
${ }^{15}$ Ibid, p17.

${ }^{16}$ Ibid, pp22-23.

${ }^{17}$ Ibid, p25.

${ }^{18}$ Ibid, p31.

${ }^{19}$ Ibid.
} 
it is our overall success that should be equalised instead. This measure is also inadequate in that it would depend on equalising different conceptions of what success actually is, and would mean that those who are more demanding on themselves in terms of measuring their own success would receive few resources relative to a person who achieves very little, but values what they do achieve. ${ }^{20}$ Lastly, Dworkin suggests that, instead of a success measure of welfare, a conception of enjoyment or pleasure might be used. However, this suffers from many of the same problems, in that it limits consideration to personal circumstances, and fails to reflect that people can and will often value states other than enjoyment, however broadly conceived, and that it is not morally wrong to do so. ${ }^{21}$

These examples demonstrate quite convincingly that personal preferences should not be the primary focus of a conception of distributive equality. There seems to be two reasons for this: firstly, that a single conception of our personal welfare is too limited in scope to satisfy distributive fairness, not only because it would exclude moral and impersonal preferences but also because one such measure cannot adequately convey the weight and complexity of even our personal circumstances; and, secondly, that some aspects of our judgments of personal welfare seem to be morally irrelevant at best, and, at worst, would clearly distort the moral value of the equality position. Dworkin concludes that approaches that attempt to equalise welfare, where expressed as some conception of success or enjoyment, are not valid distributional schemes.

Dworkin then attempts to describe a morally viable conception of resource equality.

To do this he begins with a now-familiar hypothetical situation:

\begin{abstract}
Suppose a number of shipwreck survivors are washed up on a desert island that has abundant resources and no native population, and any likely rescue is many years away. These immigrants accept the principle that no one is antecedently entitled to any of these resources, but that they shall instead be divided equally among them.... They also accept... the following test of an equal division of resources, which I shall call the envy test. No division of resources is an equal division if, once the division is complete, any immigrant would prefer someone else's bundle of resources to his own bundle. ${ }^{22}$
\end{abstract}

This outcome, Dworkin argues, suffers from two potential sources of unfairness: firstly, in the composition of the resources being distributed, and, secondly, in the way

\footnotetext{
${ }^{20}$ Ibid, p36.

${ }^{21}$ Ibid, pp43-44.

${ }^{22}$ Ibid, pp66-67.
} 
these resources are bundled together to form the endowment of each person. ${ }^{23}$ Both of these infer a multiplicity of possibilities between which the envy test is silent.

Dworkin's response to these is to propose an auction whereby each person is endowed with the same amount of money (clamshells), and where each lot can, at the request of any potential bidder, be broken down into smaller parcels. ${ }^{24}$ The prices, and size, of each lot is then adjusted until a position is reached where there is only one purchaser of each lot at those prices and all lots are sold. This process uses the auction-market to set the relative prices of resources. By this method it overcomes the difficulty of finding a meaningful equality between heterogeneous resources. The outcome is clearly recognisable as equal and fair in that each party has the same purchasing power at the auction, and the process seems to treat all individuals equally.

This auction process overcomes the second potential unfairness, in that all resources are broken down in any possible way, should an individual wish to purchase only part of them. However, the first source of unfairness is not addressed. Dworkin recognises that all will be arbitrarily constrained by the initial set of resources. A person may not particularly enjoy or desire any of the resources on offer, but this, as he puts it, is just bad luck. ${ }^{25}$ And this is not the only source of luck Dworkin recognises; because a market process is used to set prices, the bundles people are able to purchase will depend on the desires of others. Under such circumstances, having unpopular preferences would be quite beneficial. ${ }^{26}$ Dworkin recognises, then, that the moral value of this market approach to equality is undermined by the play of luck, in a number of different forms, and much of the remainder of his theory is based around identifying and reducing this deleterious effect.

Dworkin distinguishes between option luck, which represents the outcome of deliberate and calculated gambles, and brute luck, which does not involve in any way a deliberate gamble or intentional risk-taking. ${ }^{27}$ To illustrate this point, the person who develops cancer might be thought to be suffering from brute luck, although if this

\footnotetext{
${ }^{23}$ Ibid, pp67-68.

${ }^{24}$ Ibid, p68.

${ }^{25}$ Ibid, p69.

${ }^{26}$ Ibid.

${ }^{27}$ Ibid, p73.
} 
person has smoked all their life, then it might better be described as option luck. ${ }^{28}$ Dworkin uses this distinction in two initial ways. The first is to claim that equality is furthered by option luck, provided that all have a similar opportunity to take the risks involved, but is diminished by brute luck. ${ }^{29}$ The second is that the possibility of insurance links the two kinds of luck: if one could have purchased medical insurance, and yet fails to do so, then developing cancer takes on the flavour of option rather than brute luck. ${ }^{30}$

The basic structure of the remainder of Dworkin's theory can be readily derived from this analysis: Dworkin advocates a comprehensive scheme of insurance to convert brute into option luck, and to ensure that the initial equality is not compromised by the continuing operation of market mechanisms. This raises the issue of whether natural differences are to be treated as brute or option luck. Rawls' approach to the natural lottery is to correct for its effects. In doing so he sets out a theory that is difficult to reconcile with the operation of a market system. In contrast, Dworkin embraces the market mechanism, using it to define resource equality, and allowing it to affect this equality over time through the operation of option luck. Rawls might object that some are naturally more able to take advantage of option luck, and that a system that allows this is will lead to inequalities over time. Dworkin treats natural disadvantages as part of his insurance scheme.

In examining the distinction between option and brute luck, it is important to realise how natural differences are treated. Dworkin focuses on this issue by using the example of someone born handicapped - and, so, never had the opportunity to buy insurance against this unlucky outcome. He argues that in this situation, in order to preserve a fair equality, we ought to calculate the average amount of insurance that everyone would buy against this handicap and to award out of some common fund the benefit of such an insurance policy to the individual in question. ${ }^{31}$ This leads Dworkin to consider what would amount to a handicap that should be compensated for in order to achieve a morally valuable equality. People's lives are affected by all manner of differences in mental and physical attributes, many of which are unable to be traded

\footnotetext{
${ }^{28}$ Ibid, pp73-74.

${ }^{29}$ Ibid, p76.

${ }^{30}$ Ibid, p74.

${ }^{31}$ Ibid, p78.
} 
or equalised in any truly meaningful way. ${ }^{32}$ The hypothetical insurance market seeks to compensate those disadvantaged by their handicaps; not to overcome or negate these differences, but to provide them with a greater share of resources in such a way as to satisfy, and to reinforce, his conception of resource equality. ${ }^{33}$

Dworkin goes on to account for the differences between people in terms of the disequalising effect of the market. He argues that, if all people are equally talented, then the operation of the market will support the satisfaction of the envy test.

Suppose Adrian... would rather work hard at tilling his land than settle for less than champagne.... The choice should be indifferent under equality of resources, so long as no one envies the total package of work plus consumption that he chooses - so long as no one envies, that is, his life as a whole.... [This] requires that no one envy the bundle of occupation and resources at the disposal of anyone else over time, though someone may envy another's bundle at a particular time. ${ }^{34}$

In this way Dworkin rather deftly overcomes the criticisms of the inequality effect of the market by including in the envy test the effort chosen in order to gain the resources and, presumably, the risks accepted in the process, and not simply by speaking in terms of bundles and resources.

However, this approach fails in a world of unequal talents for production: the options that are available to one person may not be available to others, so that the bundle of consumption and effort that is available to one person may not be available to another. Dworkin admits that the effort involved in building one's resources does not breach the envy rule, but that the rule itself is undermined by the natural differences in talents and abilities, which ought to be adjusted for in order to preserve a meaningful equality:

We must, on pain of violating equality, allow the distribution of resources at any particular moment to be (as we might say) ambition-sensitive. It must, that is, reflect the cost or benefit to others of the choices people make so that, for example, those who choose to invest rather than consume, or to consume less expensively rather than more, or to work in more rather than less profitable ways must be permitted to retain the gains that flow from these decisions in an equal auction followed by free trade. But on the other hand, we must not allow the distribution of resources at any moment to be endowment-sensitive, that is, to be affected by differences in ability of the sort that produce income differences in a laissez-faire economy among people with the same ambitions. ${ }^{35}$

\footnotetext{
${ }^{32}$ Ibid, p80.

${ }^{33}$ Ibid, p81.

${ }^{34}$ Ibid, pp84-85.

35 Ibid, p89.
} 
The best way to implement this ambition, but not endowment-sensitive idea, Dworkin argues, involves a system of income tax and redistribution. This represents a compromise between allowing a person to benefit from the choice and the effort expended to be productive, and acknowledging the luck involved in being able to achieve, and actually achieving, productive outcomes. ${ }^{36}$

Such a system treats a lack of talent as, in effect, a disability, and applies a compulsory insurance scheme in relation to each person. But the difference between this and the earlier approach to physical handicaps is that every person is likely to suffer in some areas of their lives, or their potential lives, a substantial lack of talent. This being the case, it is not realistic to insure everyone fully against this lack; there would not be sufficient resources to compensate all for their shortfall from a state of complete talent. This type of insurance, Dworkin argues, will be viable only when much lower levels of resources are insured for. ${ }^{37}$ Ultimately, this equates to a system of income tax, the proceeds of which are distributed among those who are most lacking in resources. Such a level of insurance would be purchased by individuals were they in the position to do so, especially, Dworkin argues, given normal attitudes to risk and diminishing marginal utility of money; ${ }^{38}$ most people would make such a payment to guarantee they would always be able to afford to have some basic choices about how to live regardless of their actual level of talent. In such a system, people tend to benefit from additional money to a decreasing extent as the level of money rises, i.e. your first dollar tends to benefit you more than your thousand and first.

The overall scheme of Dworkin's equality of resources, then, involves an initial auction that serves to set the relative prices of various goods, and in which all have an equal purchasing power. Subsequently, an essentially market-based system would be applied. To the extent that shifts in resource levels reflect differences in ambition, effort and risk-taking, the envy test will continue to be satisfied; but, since these shifts also reflect natural differences in ability and talent a system of income tax and redistribution will be used so that a minimum level of income and, so, a valid conception of equality of resources would be perpetuated.

\footnotetext{
${ }^{36}$ Ibid, p91.

${ }_{38}^{37}$ Ibid, p97.

${ }^{38}$ Ibid, p100.
} 
A final point on Dworkin's resource equality concerns the role of liberty, as discussed in an essay published six years later. Here the liberties are viewed in respect to equality, although the relationship between them is developed further:

So liberty is necessary to equality, according to this conception of equality, not on the doubtful and fragile hypothesis that people really value the important liberties more than other resources, but because liberty, whether or not people do value it above all else, is essential to any process in which equality is defined and secured. That does not make liberty instrumental to distributional equality any more than it makes the latter instrumental to liberty: the two ideas rather merge in a fuller account of when the law governing the distribution and use of resources treats everyone with equal concern. ${ }^{39}$

Dworkin's original overarching idea of equal concern and respect is not inconsistent or contrary to a focus on liberties, but, rather, some liberties are essential to his scheme, not as necessary consequences of distributive equality, but as part of the very fabric of equality.

\subsection{Liberalism and equality}

Dworkin's project is to examine the distributive consequences of holding an equal concern and respect to be the central ethical principle. The general ideal here, and one that has influenced subsequent liberal theory, is that a just distribution should allow individuals to make choices according to their own conceptions of how to live, but should compensate for differences in their circumstances. The justification of this equality-centred approach stems from Dworkin's requirements of a description of the constitutive morality of liberalism, as set out above. Essentially, this means that it must represent the best account of liberalism's moral core. His later exploration of resource equality is not supposed to contribute anything further to this justificatory approach. In the introduction to the account of resource equality he states that he is "occupied, for the most part, simply in defining a suitable conception of equality of resources, and not defending it except that such definition provides a defence". 40 Although he cannot be understood as providing a full justificatory account for resource equality, it is clear that he understands equality to be a substantial part of the

\footnotetext{
${ }^{39}$ Ibid, pp122-123.

${ }^{40}$ Ibid, p65.
} 
core of liberal moral belief. This means that the resulting system must be recognisable as both liberally and ethically valuable, and, so, examinable on these terms.

Dworkin did publish a later essay purporting to examine the foundations of his theory of resource equality. In it he identifies a particular liberal model of the good life that he argues is consistent with, and strengthens, the case for his theory. This 'challenge model' consists of individuals selecting and facing challenges, and promotes skilful performance on one hand, and justice and ethical integrity on the other. ${ }^{41}$ Dworkin hopes to describe a model of the good life that accords with or implies his resource equality, and is itself recognisable as a liberal expression of what it means to live well. This account would seem to be undermined in a familiar way: it is plausible, yet ignores alternative aspects of the good life that are equally plausible or valued, and the potential complexity that a general account of the good life might reasonably involve. ${ }^{42}$ Whether this accusation is well-founded or not, the challenge model offers little by way of a justificatory foundation for his resource equality. This means that the latter can best be examined on its own terms, as an important part of liberal literature aimed at defining a morally valuable conception of liberal equality.

Dworkin's equality of resources has two separate equalising mechanisms: the envy test and the insurance market. The former, which is satisfied when no person prefers the bundle of resources belonging to any other person over their own, does not actually use the emotion of envy, in the sense of being a discontented longing or painful awareness; nor would it be reasonable to focus a system of distributive justice on a single negative emotional state. Instead, the envy test is best understood as expressing the preferences of a group of people. As such, the satisfaction of the test has a number of virtues:

First, it conforms to a widely held egalitarian view that an individual is disadvantaged if she enjoys less wealth than others because of the circumstances in which she lives ..., rather than because of her ambitions.... Second, the envy test's reliance on preferences in establishing whether inequality exists satisfies the liberal concern that justice must be sensitive to the diverse lifestyles pursued by different individuals. ${ }^{43}$

\footnotetext{
${ }^{41}$ Ibid, pp253, 270-271.

${ }^{42}$ E.g. R J Arneson, 'Cracked Foundations of Liberal Equality' In J Burley, Dworkin and his Critics: With Replies by Dworkin (2004), p81.

${ }^{43}$ M Clayton, 'Liberal Equality and Ethics' (2002) 113 Ethics 8, pp8-9.
} 
The envy test reaches an outcome that is recognisably equal, in that no person would switch with another, yet allows for various goods, ambitions and choices. In this way, it respects the choices of individuals, as alluded to in the first virtue identified by Matthew Clayton above, while carving out a recognisably liberal space within which each individual has the freedom to act as they choose. The test also removes the effects of bare circumstance on our resource bundles, in that any significant windfall for an individual would upset the envy-free balance. This result is achieved in Dworkin's auction situation by the equality of initial purchasing power. Any residual unfairness, such as the unequal distribution of talents, is then dealt with by the insurance mechanism.

The preferences expressed in the envy test can be subjected to Dworkin's own distinction between impersonal and political preferences, and personal ones. Because individuals are concerned only with the value of various bundles of resources to them, and not with a bundle as the possession of another individual, it is clear that the preference involved is entirely personal in nature. Dworkin has criticised personal preferences as the basis for welfare equality. As part of a system of resource equality, however, these preferences are incorporated differently, in that the equality that emerges from the envy test comes from the lack of preference for the resource bundles of others, rather than from the equalisation of an abstract conception of preference fulfilment. This does not eliminate the effect of a distorting belief or choice, but it does at least limit its effect to the person's bundle preferences, and to the way their resource bundle is used. In other words, because the approach equalises resources rather than welfare, distorted preferences will be limited to the legitimate purchasing power of the relevant individual. That the individual is able to prefer and use these resources as they wish, that they are able to express their preferences in this way, actually affirms the ideal of choice that underlies Dworkin's system. For this reason the auction and the envy test successfully minimise, if not avoid, the problems that welfare equality faces.

Overall, Dworkin's envy test produces a result that is recognisably liberal and fair in the face of both homogeneous goods and varied preferences and ambitions. It can also take into account an individual's deliberate risk-taking behaviour, and their attitude towards leisure and work, by including these into the resource bundle to be compared. 
However, as Dworkin admits, it cannot by itself adjust for brute luck and circumstance, different endowments of talent, and the dynamic shifts inherent in the operation of the market. In each of these situations an insurance system will operate to charge each person the premium relating to the level of coverage they choose, or would have chosen, and pay out as is appropriate. By this approach, brute is converted into option luck, which furthers rather than distorts equality and promotes the role of choice in distributive justice. It is this move that has drawn the most criticism of Dworkin's theory and has led to it being labelled as 'luck egalitarianism'. One of the main criticisms is that the insurance system could not work in the straightforward and seemingly fair way that Dworkin envisages.

His approach rests on two crucial assumptions: firstly, as to the circumstances that people would choose to insure against, and, secondly, as to the likely level of coverage. In terms of the first assumption, Dworkin identifies disabilities and lack of talent as the two general states against which individuals would insure. Yet, it is possible that additional coverage would be chosen. Phillipe van Parijs argues that this system might lead to compensation for expensive tastes - the incorporation of which is one of Dworkin's criticisms of welfare equality.

Suppose you and I have identical internal endowments, including a pathetic disposition for playing the oboe. I am stubbornly sticking to the ambition of becoming a brilliant oboe player, whether for its own sake or because of the fortune I believe I could earn this way. You instead have wisely shifted your aspirations to table soccer, which we are both far more gifted for. ${ }^{44}$

According to van Parijs' argument, the oboe player would be able to insure against their taste for playing the oboe turning out to be costly, as a result of their lack of talent. This would mean compensation for an expensive taste, which is against the intuitive beliefs of both Dworkin and van Parijs.

Dworkin explicitly replies to this argument in saying that

[1] ow wage insurance insures against being unable to earn at the level the insured party specifies in the insurance contract. If we both specify the same earning level, if we both have the same talents, and if these talents do not enable us to earn at the stipulated level, then we will both collect the same indemnity in spite of my stubbornness about the oboe. ${ }^{45}$

\footnotetext{
${ }^{44}$ M Van Parijs, 'Liberty, Equality, Envy and Abstraction' in Burley, op cit, above note 42, p53.

${ }^{45}$ Dworkin in Burley, op cit, above note 42, p352.
} 
Dworkin argues, then, that what individuals are compensated for is a lack of endowed talent and ability as manifested in a lack of earning capacity or opportunity. Because the decision to stubbornly persist in playing the oboe, despite a lack of talent, does not affect that person's earning opportunity, he or she will receive the same compensation as his or her table soccer-playing twin. Dworkin's insurance scheme, then, is restricted to compensating for a lower earning capacity caused by lesser talents and abilities. This achieves the desired effect of correcting for unfair outcomes of what Rawls would call the 'natural lottery', but not for the choices people make about how to live. As such, it is robust enough to counter arguments about other uncertainties or problems against which individuals might conceivably want to insure on the grounds that these do not concern the unfairness that the scheme is supposed to correct, as manifested in terms of earnings or resource potential. In this way van Parijs' criticisms are unfounded.

The next issue concerns the level of insurance. Dworkin's system depends on this level being minimal. In this way he avoids the enslavement of the talented: where those with few talents receive a high level of compensatory income, while talented individuals are forced to spend much of their time working, in order to be able to pay the high premiums that such a system would require. A number of commentators have criticised Dworkin's insurance market on the grounds that he does not effectively prevent this form of unfairness. Robert van der Veen, for example, argues that the hypothetical insurance decision does not in itself imply a low-coverage result, and that this choice will depend on each person's hypothetical risk preferences. ${ }^{46}$ It is possible to explain and test Dworkin's insurance market using a graphical example that represents a possible society.

\footnotetext{
${ }^{46}$ Van Parijs, op cit, above note 44, p74.
} 


\section{Resource-Equalising Insurance Market}

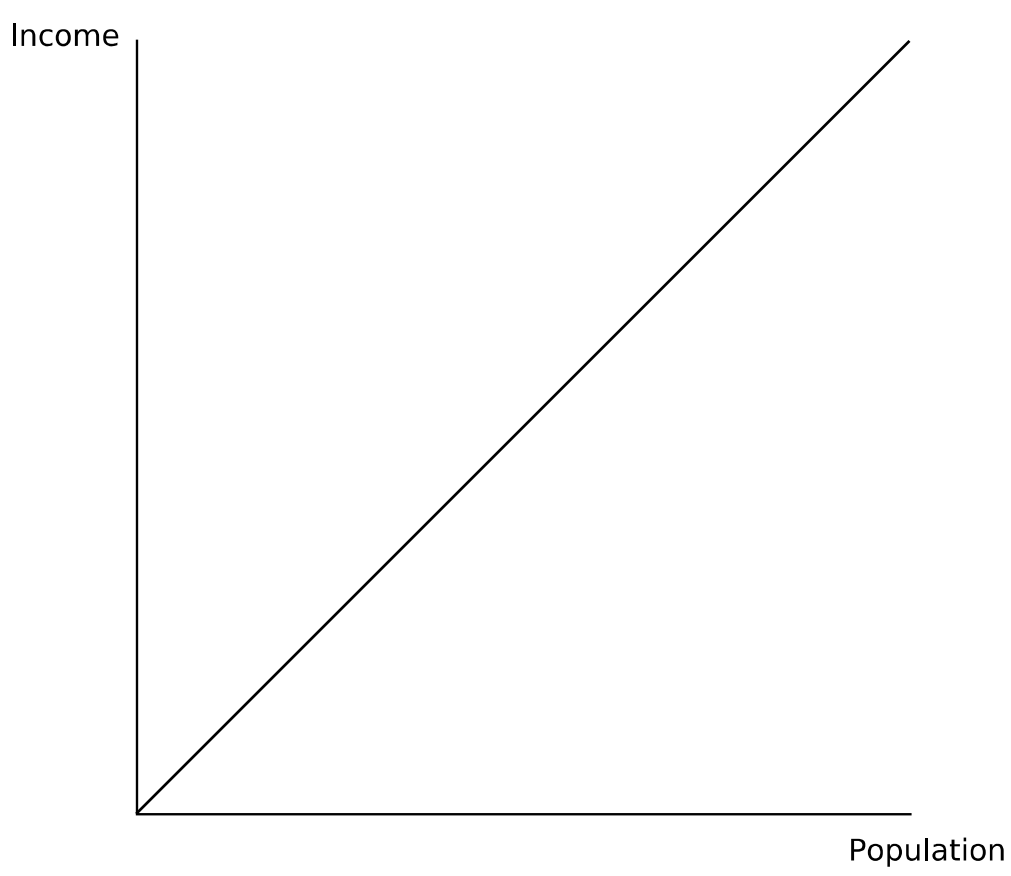

This graph shows a society where the income opportunities (represented on the vertical axis) are distributed regularly across the population. The horizontal axis records the population level, which is, in effect, ordered by their income opportunities. The 45 degree line shows that these opportunities are distributed evenly from the lowest to highest income. This would be satisfied, for example, by a society where there is one, and only one, person with the opportunity to earn each income level from, say, $\$ 1$ to $\$ 1 \mathrm{~m}$. Such a distribution is unrealistic, but the graph still allows some basic points to be made about Dworkin's scheme. In particular, the fact that the population is represented in this way allows geometrical areas to be used to represent total amounts. In the above graph, for example, the total area under the income line represents the total income opportunity for society as a whole. 


\section{$50 \%$ Income Coverage}

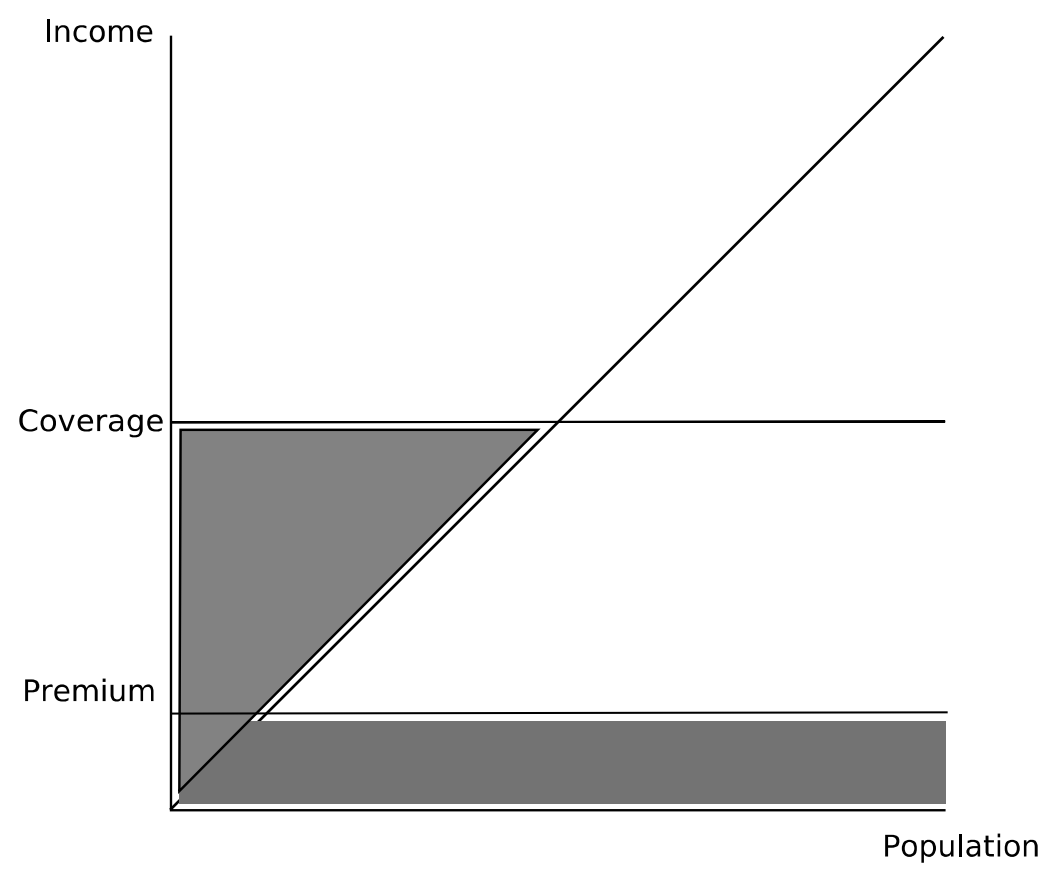

This graph demonstrates the insurance scheme in the above-described society, where each person chooses to insure at $50 \%$ of the maximum income level, as shown by the line of coverage. The shaded triangle represents the amount of compensation that would be paid to those whose income opportunity is below the coverage level. The person with the lowest income, represented at the very left of the graph, for example, is compensated by virtually the entire coverage level. This compensation needs to be funded by a premium. This amount is shared evenly between everyone, since all have adopted the same level of insurance. This premium is represented by the shaded rectangle, whose area is equal to that of the triangle. Any possible profit from the insurance is ignored, but this should not alter the problem significantly.

The total income opportunity, net of both compensation and premium, is represented by the dotted line in the following graph. It shows a minimum net income equivalent to the level of the coverage minus the premium, which is greater for those who have an income opportunity higher than the level of coverage. The point labelled A is where the income opportunity line crosses the net income opportunity line. The person here falls short of the coverage level, and, so, is compensated, but by the exact 
amount of the premium. Overall, their income opportunity is not altered by the insurance.

\section{$50 \%$ Income Coverage}

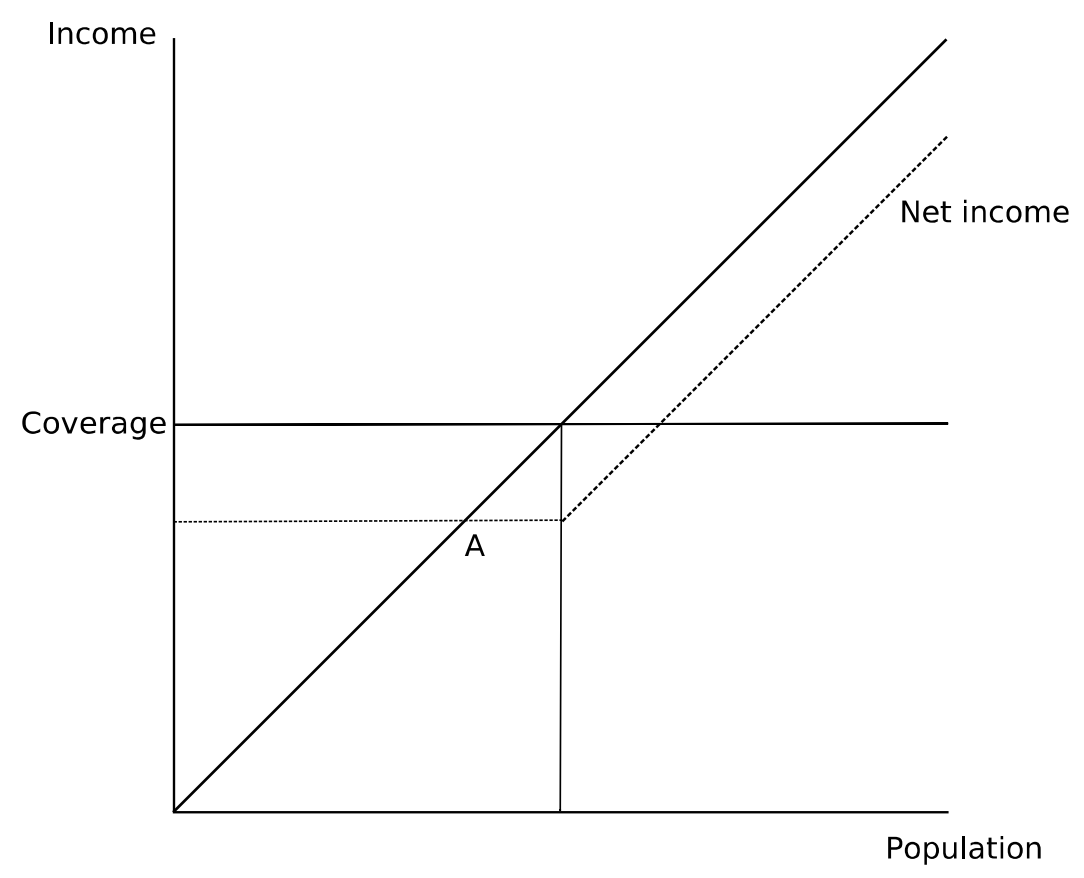

This level of coverage means that there is a minimum level of income (opportunity). This is achieved by a system that is talent-insensitive yet ambition-sensitive, and, so, according to Dworkin's analysis, is fair. It is talent-insensitive in that it equalises the net income opportunities of individuals regardless of their talent. This is most pronounced on the horizontal part of the net income line, but there is also a lesser equalising effect on the remainder of the graph, because of the effect of the premium. It is ambition-sensitive in that the net income line represents opportunities to earn; people have the choice of how hard to work, and whether and how to take their opportunities. The question that needs to be answered, then, is why it is unfeasible to have a level of coverage that maximises the equalising effect of the insurance market. This is shown in the following graph, where the level of coverage is equal to the maximum income. This means that, for this society, the premium and net income levels are equivalent at $50 \%$ of the maximum income level. In this way, the system is fully equalising, and each person has the opportunity to earn at the $50 \%$ level. 


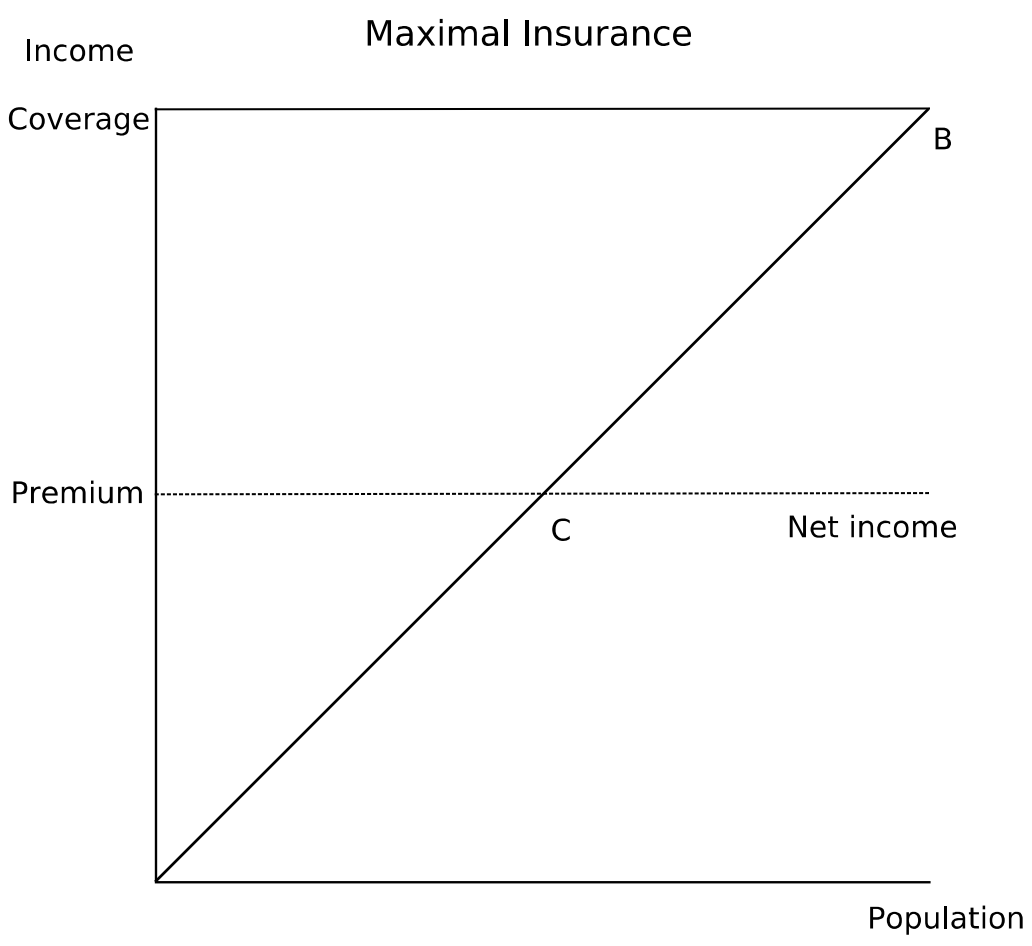

Dworkin makes a number of objections to this level of coverage, and questions its effect on welfare:

\begin{abstract}
Since... the chances of "winning" are extremely high - very few immigrants will turn out to have the maximum earning power - the cost of the premium will be extremely high as well. It will approach the value of the projected return if the risk eventuates. So someone who buys this insurance faces an extremely high chance of gaining very little. Suppose he loses, however; suppose he is one of those who does have the maximum earning power. He is now in much worse position than if he had never insured, because he must now work at close to his top earning capacity just to pay the high premium for his insurance on which he collected nothing - just, that is, to break even. He will be a slave to his maximum earning power.... [T] his insurance decision would be the... financially disadvantageous bet of a very small chance of a very great loss in return for the very large chance of a vary small gain. ${ }^{47}$
\end{abstract}

This analysis is undermined by the above graphical representation of the maximal insurance market. Although there is a very high premium, namely half of the level of coverage, half of the population are actually advantaged by compensation. In this way, instead of facing a very large chance of gaining very little, people have an even chance of augmenting their income opportunity so that it is equivalent to the average earning potential. On these terms the insurance does not seem such a poor bet.

\footnotetext{
${ }^{47}$ Dworkin, op cit, above note 10, pp96-97.
} 
The person with maximum earning power, at point $\mathrm{B}$, is not in a great situation, but certainly is not as damned as Dworkin insists. He or she must pay half his or her potential earnings in insurance premium; anything beyond that is retained as usable income. It is true that he or she has to take his or her maximum earning opportunities in order to reach the $50 \%$ income level, but that is equally true of the person at point $\mathrm{C}$ who receives no net assistance from the insurance scheme. The main difference between the two is that the leisure time for the person at $\mathrm{C}$ is cheaper than the maximally earning person - but that is also true regardless of any insurance. Here, the risk of being the person at point $\mathrm{B}$ is not so great as to absolutely overcome the benefits from maximal insurance, namely knowing that, no matter where a person falls on the income profile, they will be capable of earning to a level that is half that of the maximum. A decision to purchase this insurance is not completely irrational, as Dworkin suggests. Consequently, his conclusion that a minimal or subsistence level of insurance would be appropriate is unwarranted.

It may be that the slavery-of-the-talented result is more easily reached when income opportunity is less equally distributed. If, for example, there are one hundred people who have income opportunities of 1 , and one person who has the opportunity to earn of 100 , then that person will have to pay a premium of just over 98 in a system of maximal insurance. But, even here, it may be that people are willing to take the risk that they will be one of the hundred, whose income opportunity is almost doubled by the insurance.

Overall, then, Dworkin's system of minimal insurance has a number of problems. Firstly, the slavery-of-the-talented result seems to depend on the nature of the distribution of talents, and, in particular, on the shape of the distribution of income opportunities. But, whatever this distribution, the idea that people would reasonably agree to only a minimal level of insurance is not sustainable. In fact, a system that is more talent-insensitive and resource-equalising seems quite a possibility. Given that these are important aims of Dworkin's theory, it seems unusual that he would not recognise and embrace this. What Dworkin may actually be concerned with is avoiding the result that the talented are disadvantaged, that they cannot do better than the untalented, and that the more talented a person is the more the product of their 
talent is appropriated for the general good. This contrasts strongly with the Kantian ideals of both Rawls and Nozick, where a person cannot be used simply for the good of another. Seen in this light, Dworkin's insurance starts to take on some of the characteristics of utilitarianism, a point that has not escaped the notice of critics. ${ }^{48}$ One final difficulty with Dworkin's proposed minimal insurance scheme is its uncertain relationship with the envy test. Because minimal insurance implies a minimal level of compensation, and some residual inequality based on differences in talent, it will not result in a resource distribution that satisfies the envy test.

What is unclear is how the insurance scheme and envy test each contribute towards greater overall resource equality. A number of commentators have identified this tension between the two as a substantial fault in Dworkin's theory. ${ }^{49}$ According to Dworkin's narrative the envy test is applied to the original auction that is undertaken by the shipwreck survivors. At that point the insurance system takes effect in order to compensate for talent differentials. The problem is that if the envy-free and resourceequal position is progressively undermined, then over time it seems to serve no purpose at all. Dworkin seems to reconcile the two by arguing that the envy test is satisfied at the point at which the insurance decision is taken, ex ante, and that " $[\mathrm{t}] \mathrm{rue}$ equal concern requires ex ante, not ex post, equality". ${ }^{50}$ Michael Otsuka rejects this conclusion by arguing that ex ante equality does not represent a morally fair position. His arguments seem to depend primarily on the idea that the insurance coverage need not, and, in fact, will usually not, be fully compensating. Dworkin's insurance system, he argues, is unfair to those severely incapacitated, who will only be partially or minimally compensated for their loss. The choice to insure does not, in any reasonably fair way, transform the brute bad luck of going blind, say, into the morally-acceptable bad option luck. ${ }^{51}$

Otsuka also uses the example of a horrible and incurable mental illness. ${ }^{52}$ If nothing at all can be done to help the unfortunate victim of such an illness, then they will be the

\footnotetext{
${ }^{48}$ M Fleurbaey, 'Equality of Resources Revisited' (2002) 13(1) Ethics 82, p96.

${ }^{49}$ M Otsuka, 'Luck, Insurance and Equality' (2002) 113(1) Ethics 40, p42; R van der Veen, 'Equality of Talent Resources: Procedures or Outcomes?' (2002) 113(1) Ethics 55, p56; Fleurbaey, op cit, above note 48.

${ }^{50}$ R M Dworkin, 'Sovereign Virtue Revisited' (2002) 113(1) Ethics 106, p124.

${ }^{51}$ Otsuka, op cit, above note 49, p45.

${ }^{52}$ Ibid, p50.
} 
victims of bad brute luck. This result, he argues, does not reflect the ideal of resource equality. ${ }^{53}$ But, if there is some procedure that is expensive but only minimally effective at alleviating the symptoms of the illness, then each person will have the opportunity of insuring for this procedure. This, it seems, turns the brute luck into option luck, and makes the situation ex ante equal and fair. Otsuka argues that this change, from unfair to fair, is not justified simply by the change in circumstances, and does not actually reflect an equality of resources. Finally, he compares this to the situation where the illness is curable, and at a minimal cost. In this situation the insurance system is fair, as each person will have the reasonable opportunity to insure against the whole of the loss that they would potentially face. ${ }^{54}$

In fairness to Dworkin, his insurance system is concerned primarily with compensating for a diminished opportunity to earn income, whether through disability or want of talent; the effect of resource-costing cures on disability insurance is outside the scope of his system. But the reasoning behind Otsuka's argument remains, that partial compensation insurance does not deal adequately with disadvantage, and that ex ante equality does not reflect adequately the ideal of equal concern and respect. Expressed in this way, his general argument seems quite convincing.

Dworkin's theory of resource equality as a whole, then, does not seem to achieve its own objectives. It consists of an envy test that cleverly results in a position that is recognisably equal, and that is sensitive to differential goods as well as individuals, in their desires, preferences and plans. It is also able to incorporate different decisions by people as to leisure, and the amount of effort and enterprise expended in income earning. In order to compensate for what Rawls would call the natural lottery, Dworkin augments the envy test with an insurance scheme. The resulting inequality, however, cannot be justified by the hypothetical choice to purchase partiallycompensating insurance. This choice is, in effect, an expression of risk assessment in ignorance of a person's actual position, and is not recognisably equalising. Dworkin might argue that it is fair or reasonable, in the context of scarce resources, or because it respects individuals; however, it does not hold resource-equality as its first virtue. This virtue would imply a fuller compensatory scheme and more obviously egalitarian

\footnotetext{
${ }^{53}$ Ibid.

${ }^{54}$ Ibid.
} 
results, but would also mean that the talented would be disadvantaged and, to some extent, used by the remainder of society. It could be argued that equal concern and respect would go against this result, but Dworkin is explicitly concerned with how this equality is expressed in relation to resources. What this may actually indicate is the complexity of such a nebulous concept as equality, and of the variety of political structures that might be required to express it adequately, in order to avoid the unattractive consequences of any one approach.

\subsection{Equality revisited}

In the preceding chapters there were found to be a number of similarities between the theories of Rawls and Nozick, in that both employ complex theoretical structures in order to reveal a morally acceptable theory of justice. In both cases, this is carried out in the context of a moral priority: for Rawls, the priority of right over the good and, for Nozick, natural rights. These reflect the Kantian ideal of the individual as an end and not a means of value, and they operate to allow individuals to pursue their versions of the good life within the corresponding liberal guidelines.

Dworkin's system loosely employs a moral priority, namely equal concern and respect, expressed in a resource context. It also defines a liberal space within which individuals can choose how to act: the choice to earn and spend resources however one pleases within the guidelines implied by the system and, in particular, within the adjustments implied by minimal income insurance. What stands out about Dworkin's system is its focus on comparisons of resources, rather than on any overarching rights, and on equality as the central liberal concern. The substance of the system itself faces a number of technical problems, as did those of both Rawls and Nozick, but the shift implied by Dworkin's theory is one away from rights-centred approaches. This approach was quite significant in the development of liberal thought, and led to a number of competing theories focussed on defining a morally valid expression of liberal distributive equality.

Each of these constructs a different conception of equality. 
Luck egalitarians disagree with one another primarily over the space in which they advocate equality. Should egalitarians seek equality of resources or assets (Dworkin, Rakowski, Roemer), real freedom - that is, legal rights plus the means to achieve one's ends (van Parijs), equal opportunity for welfare (Arneson), or equal access to advantage - a mixed bag of internal capabilities, opportunities for welfare, and resources (Cohen, Nagel)? This looks like a wide diversity of views, but the central disagreement among them separates luck egalitarians into two camps: one which accepts equality of welfare as a legitimate ... object of egalitarian concern $\ldots$ and one which only equalises resources. ${ }^{55}$

Elizabeth Anderson categorises these theorists using Dworkin's original distinction between equality of welfare and resources. It is not clear that any of these has managed to overcome, either, the arguments against welfare-equality identified by Dworkin, or, the arguments levelled against resource equality. Issues such as expensive tastes and the slavery-of-the-talented seem to persist against these theories. $^{56}$

Two more arguments are interesting in this general way. Firstly, Samuel Scheffler has criticised the focus of these theories on giving positive expression to the distinction between choice and circumstance. Scheffler argues that a liberal equality ought to allow resource differences brought about by the choices of individuals, but should correct for those that are the consequence of mere circumstance. The division between choice on the one hand and circumstance on the other he calls both "philosophically dubious and morally implausible". ${ }^{57}$ The distinction is difficult, and brings into question the meaning of free choice and voluntariness, and whether there is any meaningful and concrete distinction between this and unavoidable circumstance. When a choice is made because of a person's identity, which is itself an amalgam of some kind of choice and circumstance, it is difficult to see the sense in which it is free and voluntary, and even more difficult to imagine basing a political system on this idea. ${ }^{58}$ This general argument is compelling in that it forcefully attacks the very distinction that informs all of these liberal approaches to distributive equality.

A second general criticism is that they focus on resource distribution to the exclusion of all else. This would seem to be behind Anderson's argument that

\footnotetext{
${ }^{55}$ E S Anderson, 'What's the Point of Equality?' (1999) 109(2) Ethics 287, p293.

${ }^{56}$ A Stark, 'Beyond Choice: Rethinking the Post-Rawlsian Debate over Egalitarian Justice' (2002) 30(1) Political Theory 36, pp39-41.

${ }_{57}^{57}$ S Scheffler, 'What is Egalitarianism?' (2003) 31(1) Philosophy and Public Affairs 4, p17.

${ }^{58}$ Ibid, p18.
} 
[i]n focussing on correcting a supposed cosmic injustice, recent egalitarian writing has lost sight of the distinctively political aims of egalitarianism. The proper negative aim of egalitarian justice is not to eliminate the impact of brute luck from human affairs, but to end oppression, which by definition is socially imposed. Its proper positive aim is not to ensure everyone gets what they morally deserve, but to create a community in which people stand in relations of equality to others. ${ }^{59}$

She argues that the focus on distributive equality and away from personal and political rights is mistaken. Such an approach does represent a narrowing of the liberal focus that excludes an independent and prior conception of rights, and concentrates wholly on correcting the unjust effects of the natural lottery and other circumstances on one's welfare or resource bundle. This narrowing is unfortunate in that it has taken liberal theory away from constructing an acceptable justificatory structure for liberal institutions or ideals, in favour of calculating a plausible and common conception of equality. That even this task has led to a number of unanswered and perhaps unanswerable technical problems, and persistent disagreement between its practitioners, is perhaps the most interesting result of the meticulous industry involved.

Social economist Amartya Sen has also participated in this debate, and supports a conception of equality based on human capabilities. In reaching this conclusion, he makes a number of general comments about equality as the focus of a political theory. He argues that every liberal theory, and in fact every normative theory of social arrangement, requires some form of equality. ${ }^{60}$ The question then becomes, not why equality, but equality of what. ${ }^{61}$ In exploring this question, Sen notes two important ideas: firstly, that equality in one space will tend to lead to inequality in another; and, secondly, that a complicating factor in approaching equality is our inherent and substantial diversity. ${ }^{62}$

Sen's response to these difficulties is to develop a conception of equality based on our capability to function. Functioning, he states, consists in our being and doing.

Closely related to the notion of functionings is that of the capability to function. It represents the various combinations of functionings (beings and doings) that the person can achieve.

\footnotetext{
${ }^{59}$ Anderson, op cit, above note 55, pp288-289.

${ }^{60}$ A K Sen, Inequality Reexamined (1992), p13.

${ }^{61}$ Ibid, p16.

${ }^{62}$ Ibid, p28.
} 
Capability is, thus, a set of vectors of functionings, reflecting the person's freedom to lead one type of life or another. ${ }^{63}$

Our capabilities are basically the opportunities we have to be or do things. They are valuable both in that they represent the freedom to function, and that functioning depends on them. ${ }^{64}$

In many ways this is a natural end to the egalitarian strand of thought. Here, the conception of equality (albeit welfare equality) has become more abstract, and more encompassing of diverse ideas of a valuable life, but contains less concrete meaning and a lesser potential for measurement. In such a system the content of equality diminishes; at the very extreme we would seek the equality of an explicitly undefined 'valuable life', with no means of recognising that life or of equalising different lives. Such an approach might be largely agreed to, but its prescriptions would be entirely empty. Even then, there would still be some who would argue, not without some truth, that 'valuable' presupposes a conception of life that is not universally shared.

It seems that either a liberal conception of equality is well-defined and contestable or so broadly conceived as to have no definite meaning. This result is entirely concordant with the view of equality as an open and undefined term, one that has meaning, but not one that accords with a single conception, as well as with the view that there is no correct or best liberal conception of equality that we can discover by application of hypothetical situations and rational structures. It not only matches the morally uncertain approach that criticised the priorities inherent in the theories of Rawls and Nozick; it also questions the idea of a liberal theory being found from some constructed conception, be it of justice, natural rights or equality. In other words, it questions liberal theory as a project whereby a single theme is conceived, built, and given content from a theoretical structure. Although some form or expression of material equality will be important to any liberalism, equality itself does not seem to be the key to understanding a universally recognisable scheme of liberal justice.

\footnotetext{
${ }^{63}$ Ibid, pp39-40.

${ }^{64}$ Ibid, pp40-41.
} 


\title{
3. Ackerman and neutrality
}

\subsection{Neutral liberal dialogue}

In his book, Social Justice in the Liberal State (1980), Bruce Ackerman attempts to build a complete liberal theory on a significant justificatory structure. As with $A$ Theory of Justice, be begins from a hypothetical narrative that pares away many of the complicating and distorting layers present in any real society, in order to reach some general and universal conclusions about liberalism.

\begin{abstract}
Imagine that you and I (and the rest of us) embark on a voyage of discovery, forsaking our previous wealth and position to enter upon the quest. Coming unexpectedly upon a new world, we scan it from afar and learn that it contains only a single resource, manna, which has some remarkable properties. Most important, manna is infinitely divisible and malleable, capable of transformation into any physical object a person may desire. Further scanning reveals, however, that... there won't be enough manna to satisfy the total demands of all the members of our party. A struggle for power is inevitable. ${ }^{65}$
\end{abstract}

As the spaceship approaches the planet a conversation ensues as to how the manna will be divided amongst the population. The Commander of the spaceship presides over this conversation in order to ensure that a fair and legitimate outcome is reached. To achieve this she employs two rules, the first of which is rationality: for a rule that effects the distribution of manna to be effective it must "specify a power structure in which each power wielder can support his claim to manna by giving a reason when challenged by any of his fellow citizens.",66

What is then required is a way of distinguishing legitimate from illegitimate reasons. This is dealt with by the second rule: neutrality. Ackerman conceives this idea as having two parts: firstly, the bar against selectivity, so that no speaker can say that their conception of the good is intrinsically superior to anyone else's. ${ }^{67}$ This means that one person's desire for manna in order to build cathedrals does not overcome the desire of another to keep his belly warm. ${ }^{68}$ The second part of the neutrality condition is the bar against unconditional superiority, so that no speaker can base a claim of superiority simply on the fact that they are making the claim, or by virtue of some

\footnotetext{
${ }^{65}$ B A Ackerman, Social Justice in the Liberal State (1980), p31.

${ }^{66}$ Ibid, p36.

${ }^{67}$ Ibid, p43.

${ }^{68}$ Ibid, p44.
} 
feature they possess. So, neutrality simply means that no argument can succeed that involves the claim of superiority of the claimant themselves or for their conception of the good.

Given these prohibitions, and the desire not to starve on board the spaceship, Ackerman identifies material equality as an appropriate and legitimate starting-point.

This stems from the idea that we are all morally autonomous beings:

\begin{abstract}
Our claims to manna can be based on nothing more - and nothing less - than a dialogic exchange in which each of us describes himself as a morally autonomous person capable of putting a value on his life plan. Given this self description, it follows that something can be said on behalf of initial material equality. While I cannot say that I' $m$ an unconditionally better person than you are, Neutrality does not forbid me from claiming rights based on a description of myself as a person whose claim to moral autonomy is at least as good as that of my competitors: Since I'm at least as good as you are, I should get at least as much of the stuff we most desire - at least until you give me some Neutral reason for getting more. ${ }^{69}$
\end{abstract}

This is the central result of Social Justice: the condition of neutrality forbids many of the distributive arguments that could be made, and, by default, what remains is the claim that I am at least as good as you and, so, I should receive at least as much as you do. This leads inevitably to pure material equality, as represented by the equal division of manna. This result permeates the whole of Ackerman's theory; and, in fact, the remainder of its substance is concerned with identifying the various liberal rights that are necessary for the continuation of such a system and for putting the theory into practice. Included in this are discussions to two closely related and quintessentially liberal concerns: taking account of the natural lottery, and the distorting effect of free exchange on a distributive equality.

Dworkin devised a conception of equality to deal with talent differentials and natural disadvantages. Ackerman's approach is quite different. He imagines a situation where the passengers on the spaceship could genetically manipulate their offspring so as to exclude certain types of people. He argues that, if every passenger agreed to exclude a type of person on the grounds that they would be at a relative disadvantage in pursuing their own conception of the good life, then that person should be genetically excluded from the population. ${ }^{70}$ A possible example of such an excluded type is a

\footnotetext{
${ }^{69}$ Ibid, pp57-58.

${ }^{70}$ Ibid, pp115-116.
} 
person who is deaf, blind and crippled. ${ }^{71}$ Such a person is said to be genetically dominated by the rest of society.

But the diversity of individuals' conceptions of the good would mean that this situation would be very rare. The much more common outcome of a comparison between genetic types would be that of an undominated diversity, where a genetic endowment is held to be better than at least one other person by at least somebody. ${ }^{72}$ This, Ackerman argues, marries his liberal equality with a diversity of people:

Rather than some nightmare identity, liberalism affirms an ideal of undominated equality, where very different human beings have an equal right to use material resources to pursue the ideals that seem best to them. ${ }^{73}$

Instead of using an insurance scheme to adjust for deficiencies in talents and abilities, Ackerman argues that, in most cases, natural difference should have no effect on distributive shares. This is because neutrality prevents the supposedly inferior person from claiming more than the same share as everyone else, since at least one other person believes that they are better endowed than some other person.

The second liberal concern relates to the effect of free exchange on distribution of resources over time. Ackerman exploits the potential technological advancements of his futuristic society to devise the idea of transactional flexibility; a situation that allows individuals to negotiate, enter and exit agreements at zero cost. He uses this to answer a number of criticisms of liberal systems, for example the bias in favour of individual over collective property. With costless negotiation and exit, any group can easily decide to pool their resources and live collectively, and this arrangement would be perfectly defined without expense. ${ }^{74}$ Also, if one person decides to leave the group they could exit without cost, taking an appropriate proportion of the resources with them. This technology would also promote the liberal ideal of free speech and free competition. Because it involves a costless and perfect communication device, everyone would have the ability to communicate with other people as they choose no government could curtail this communication, and any recipient could block

\footnotetext{
${ }^{71}$ Ibid, p117.

${ }^{72}$ Ibid, p120.

${ }^{73}$ Ibid, p121.

${ }^{74}$ Ibid, p173.
} 
communication from anyone else. ${ }^{75}$ This ubiquitous and costless communication would also facilitate exchange and competition between people, and prevent any attempt to exercise monopoly power through having privileged access to methods of communication. ${ }^{76}$ In this way, Ackerman's approach actually facilitates exchange by providing a technology that is itself neutral between individuals.

The resulting society, shaped by the neutral liberal dialogue, Ackerman believes to be a harmonious vision of social order:

\begin{abstract}
The harmony is of a distinctive kind. It does not promise an end to ambiguity, disagreement, disappointment; it does not promise an idyllic social union where all mankind loses itself in lyric praise of cosmic order. Instead ... it offers each citizen the chance to achieve selfunderstanding without subordinating himself to the meanings imposed by others; it bids us glimpse a deeper harmony in the dialogue that provides the social foundation for all subsequent disagreement. ${ }^{77}$
\end{abstract}

This is not an unfamiliar liberal sentiment, and is similar to statements made by both Rawls and Nozick. Both champion, not a single prescribed way of life, but, rather, a liberal space within which individuals can choose how to live. Distinct in Ackerman's claim is the focus on neutrality and on dialogue, which, together, are the means by which the space he envisages is conceived.

In his final chapter Ackerman sets about justifying his theory of liberalism as a neutral dialogue, and, so, this ideal liberal society. In doing so he addresses the challenges of justification. He begins by arguing that there are a number of potentially different justifications for or, as he puts it below, "paths to" liberalism. Because of these alternatives a liberal cannot blindly follow their own path, but must engage openly in conversation with others in order to find and use those other paths. ${ }^{78}$ Ackerman argues that the rules of a neutral dialogue are as valid to the conversation of liberal justification, as to that of fair resource distribution.

There is a perfect parallelism... between the role of political conversation within a liberal state, and the role of philosophical conversation in defence of a liberal state. Political talk within a liberal state is a device for organizing people who are otherwise free to follow the different paths to the good. Philosophical conversation in defence of a liberal state is a device

\footnotetext{
${ }^{75}$ Ibid, p177.

${ }^{76}$ Ibid, p178.

${ }^{77}$ Ibid, p231.

${ }^{78}$ Ibid, pp358-359.
} 
for persuading people who are otherwise free to pursue very different paths to understanding. ${ }^{79}$

Ackerman conceives moral justification as a situation of dialogue through which people can discover and explore different paths to liberal neutrality, which amount to valid philosophical justifications of the universality of liberalism. Ackerman himself identifies four such paths, each representing dialogical and philosophical routes to liberalism.

The first of these is the corrosiveness of power: the modern fact of imperial government, bureaucratic institutions and advancing technology mean that any governmental power is apt to be used mistakenly or to be abused. ${ }^{80}$ The second involves an acknowledgement of doubt as to what is good and right for mankind. ${ }^{81}$ The third path comes from the realisation that what is best for one person may not be what is best for another, and that imposing one's conception of the good on another fails to respect their autonomy and the independent value of their own conception. ${ }^{82}$ The last comes from a liberal scepticism that avoids attaching any moral meaning whatsoever to our beliefs or desires. Each of these, Ackerman argues, represents a separate explanation for, and justification of, liberal neutrality; liberal dialogue is the end-result of each of these paths, as it decentralises and equalises political power and control, recognises doubt as to the proper conception of the good and prevents one person's conception being imposed on another, and, also, responds to the actual meanings people give to their own lives.

\subsection{Neutrality and equality}

Ackerman employs the hypothetical situation of the inhabitants of a spaceship making decisions about their new world, in a way similar to the work of Rawls, Nozick and Dworkin. Ackerman's situation involves real people who are unconstrained by any veil of ignorance; the role of constraint is instead carried out by the requirements of the need to give reasons, consistency in those reasons, and the neutrality of those

\footnotetext{
${ }^{79}$ Ibid, p359.

${ }^{80}$ Ibid, pp362-364.

${ }^{81}$ Ibid, pp365-366.

${ }^{82}$ Ibid, pp367-368.
} 
reasons. ${ }^{83}$ Ackerman's focus is interesting, not because neutrality is new to liberal theory - a neutrality between conceptions of the good plays roles in both Rawls' and Dworkin's theories. ${ }^{84}$ Rather, Social Justice in the Liberal State suggests that neutrality is the central liberal concern, from which equality and various political and personal liberties are derived.

The progression from neutrality to equality is the primary mechanism of Ackerman's theory. Neutrality dictates that no-one can justify a distribution of manna by reason of a superiority of their conception of the good, or of themselves. This ultimately means that the only valid justification of a particular distribution is that each person deserves at least as much as any other, which results in a perfect material equality. Ackerman's aim here is to present a form of neutrality that is readily acceptable and recognisable to those who follow one or more of his 'paths to liberalism', and, so, who accept already the need for liberal neutrality. Perfect material equality is derived from this neutrality, as is expressed by what has been called the mantra of Social Justice: "I am at least as good as you, so I should get at least as much." ${ }^{, 85}$ The first part of this statement - "I am at least as good as you' - is supposed to stem from the observation that both hold some valued conception of the good that entitles them to some share of the manna. Ackerman argues that this does not breach the bar against claiming to be superior, or to having a superior conception of the good, since it does not imply that the maker of the statement necessarily is better than anyone else.

Dworkin identifies two possible meanings for this statement: that we are as good as each other from the standpoint of political justification; or that I am confident that noone has a better conception than I, or at least, that these conceptions can not be meaningfully compared. ${ }^{86}$ The first of these, he argues, is simply a restatement of neutrality and offers no positive argument for equality. The second can lead to an acceptable and potentially destructive reply to the claim that my conception of the good is as good as yours:

\footnotetext{
${ }^{83}$ R B Thigpin \& L A Downing, 'Liberalism and the Neutrality Principle' (1983) 11(4) Political Theory 585, p568.

${ }^{84}$ P Neal, 'Liberalism \& Neutrality' (1985) 17(4) Polity 664, p665.

${ }^{85}$ Ackerman, op cit, above note 65, p56; R M Dworkin, 'What Liberalism Isn't' (1983) 29 New York Review of Books 47, p48.

${ }^{86}$ Dworkin, op cit, above note 85, p48.
} 
No, it is not. I do not say that the superiority of my own ideals provides any argument why I should have more than you ... But of course I think my own ideals are better than yours, so there is no reason why I should accept the false proposition, that yours are at least as good as mine, as providing any positive argument for anything. ${ }^{87}$

Dworkin argues that the statement "I am at least as good as you" as a personal judgment is probably wrong, and certainly does not have the depth or power on which to base a system of justice. Bernard Williams also criticises this statement on the grounds that it reveals that Ackerman's neutrality allows some evaluations about conceptions of the good, but not others:

\begin{abstract}
Someone might really think that someone else's conception of the good was just as good as her own, and she would be allowed to say that. This shows clearly that what we're offered isn't a Neutralist restriction on the content or subject matter of our dialogues... It's a restriction that bans just some judgments and not others, although all of them belong to the same dimension of judgment. It's like being allowed to discuss the merits of the weather, so long as you don't say that today's is better than yesterday's. ${ }^{88}$
\end{abstract}

Whereas Dworkin asks why, if we believe our own conceptions to be better, we should only say that they are at least as good, Williams comments that neutrality does not exclude all judgments about the relative value of conceptions of the good. In particular, it allows us to say that someone else's conception is better.

What these arguments suggest is that the move from preventing people from claiming to be better, to the statement that "I am at least as good", is quite a jump. In the relevant dialogue, it is precipitated by the realisation that both people involved have valuable conceptions of the good, and are deserving of some material means to their achievement. This is clearly neutral between conceptions, and is not problematic.

Next, one of the speakers makes this statement:

Well, I can't say that I'm an especially deserving citizen for reasons that have nothing to do with my conception of the good. Nevertheless, the prohibition on unconditional claims of superiority does not bar me from saying that I'm at least as good as [the other person]. ${ }^{89}$

Both of these statements are also unproblematic: neutrality prevents the speaker from asserting a superior conception, yet it is open for him to say that he is at least as good as the other. It is clear that saying that he is at least as good does not breach neutrality. However, this raises the question as to why he would say that.

\footnotetext{
${ }^{87}$ Ibid.

${ }^{88}$ B Williams, 'Space Talk: The Conversation Continued' (1983) 93(2) Ethics 367, p368.

${ }^{89}$ Ackerman, op cit, above note 65, p55.
} 
Such a person might actually believe their own conception to be better, in which case, as Dworkin suggests, they are acting falsely, or at least being unnecessarily vague, like saying that three is at least as big as two. Alternatively, they might believe that comparisons valid to matters of political justice cannot be made. But, then, this statement seems unusual as a response, like saying that blue is at least as good as red. Neither of these ideas seems adequate as the basis for a theory of justice. However, this statement must be understood within the context of Ackerman's hypothetical situation, where each person is trying to maximise the resources they will receive while wanting to avoid a stalemate, and where the focus of the discussion is on the relative value of people and their conceptions of the good. Under these terms, claiming to be at least as good as everyone else is the best someone can do for themselves. It is a strange statement that seems non-neutral in one part and untrue in the other, but it is consistent with other aspects of the dialogue.

The next move is that, if I am at least as good as you, then I deserve at least as much. There is a clear link, in the context of Ackerman's dialogue, between the comparative value of a person and their proper resource allocation. Consequently, this statement, in the absence of an argument that the dialogue could take a sideways step away from interpersonal comparisons of value, seems consistent. The question it begs, as James Fishkin has pointed out, is "[a]t least as much what?". ${ }^{90}$ In deciding against using utility as the measure of equality, Ackerman's Spaceship Commander notes:

We search in vain for a neutral yardstick for measuring the "real" value of different conceptions of the good. To justify one yardstick over all the other possible ways of ranking values will require utterances that are inconsistent with at least some of the ideals affirmed by some of your fellow citizens. ${ }^{91}$

Fishkin convincingly replies that any yardstick is subject to this criticism, including using manna itself. ${ }^{92}$ There is no reason to privilege manna above utility or any other measure, simply because the ship arrives at a place where it is available and obvious. In this way, Ackerman fails to head off arguments from welfare. This has also been recognised by Larry Alexander who suggests that using the yardstick of utility might actually improve the theory: "it is perhaps more neutral to see that people receive equal utility despite their choices of good rather than to influence the choices by

\footnotetext{
90 J S Fishkin, 'Can There Be a Neutral Theory of Justice?' (1983) 93(2) Ethics 348, p351.

${ }_{91}$ Ackerman, op cit, above note 65, pp48-49.

${ }^{92}$ Fishkin, op cit, above note 90, p352.
} 
offering objectively equal goods with subjectively different yields" ${ }^{93}$ In this way, Ackerman does not overcome the difficulties that plague Dworkin, namely that equality is a complex and difficult conception with no single agreed meaning.

\subsection{Diversity, exchange and justification}

Ackerman addresses the closely linked difficulties of the natural lottery and free exchange with conceptions of undominated diversity and costless communication and exchange. The idea of undominated diversity is interesting because it reflects a moral intuition that is so completely contrary to those conceptions of Rawls and Dworkin. It rests, ultimately, on the idea that our personal abilities are plural and complex, so that it is difficult to declare that one person is better than another without implicitly relying on a conception of the good as the standard by which this is judged. The resulting idea of an undominated genetic diversity is recognisably neutral. The problem here is that it does not fit easily within Ackerman's liberal dialogue. He uses it to show why all but the most seriously handicapped genetic types should be allowed to be born. This is the case where there is at least one person who believes that a particular genetic type is better than another. ${ }^{94}$ But each of these individual judgments is clearly non-neutral, and the standard of unanimity among non-neutral judgments within a particular population, apart from itself being non-neutral and arbitrary, is not sufficiently justified as part of a neutral dialogue..$^{95}$

This approach to the natural lottery fits easily with Ackerman's promotion of free exchange. From this perspective it seems that his view is libertarian in the sense that people are entitled to the benefit of their own efforts, although he never states this explicitly. ${ }^{96}$ In fact, Ackerman, while championing free exchange, seems to contemplate neither production and productive action, nor the inequalities that may result from them over time. This has led Dworkin to comment that Ackerman's system

\footnotetext{
${ }^{93}$ L A Alexander 'Liberalism as Neutral Dialogue: Man and Manna in the Liberal State' (1981) 28 UCLA Law Review 816, p834.

${ }_{95}^{9}$ Ackerman, op cit, above note 65, p120.

${ }_{95}^{95}$ See Ackerman, op cit, above note 65, 121; Alexander, op cit, above note 93, p845.

${ }^{96}$ Alexander, op cit, above note 93, p817.
} 
has this cold message for most of those who end up with less income: you had a liberal education, you had your equal grain of manna, and no argument is available why you should be entitled to invade the manna of those more clever or more skilful or simply luckier. ${ }^{97}$

This may have been an oversight, but it is difficult to see how redistribution could be permitted in the context of a liberal dialogue between people in an undominated genetic diversity.

The final, and perhaps most interesting part of Ackerman's theory of justice is his justification of the neutral liberal state. If the derivation of equality from the conditions of neutrality is the main mechanism of Social Justice, then the outcome of the theory depends on justifying these conditions. Ackerman provides four reasons, or paths, for this in the context of philosophy as a conversation between persons. In building his conception of philosophy as a dialogue, Ackerman draws a number of parallels with the neutral political dialogue. Taken on their own, his comments on philosophical dialogue are significant:

Philosophical conversation in defence of a liberal state is a device for persuading people who are otherwise free to pursue very different paths to understanding... The task of philosophical conversation is to make it possible for a person to reason his way to Neutrality without declaring that the path he has chosen is intrinsically better than any other route to liberalism.... [R]ather than using philosophical argument to convert people to a single common understanding, liberal theory invites people to pierce their substantive disagreements and achieve a deeper unity. ${ }^{98}$

On these terms it seems that there is an exact match between philosophical and political dialogue, and that the conditions of neutrality apply to each. Patrick Neal has described this approach as being both absurd and circular:

[C]ircular, because neutral dialogue needs to be defended, not presupposed. Absurd, because we cannot prove that there is no good common to all persons as such by preventing anyone from raising the question of whether there might be in the first place. This is ordinarily called coercion, not argument. ${ }^{99}$

From Ackerman's comments it does seem that neutrality is both the condition and the endpoint of philosophical dialogue, so that anything not relevant to showing that neutrality is appropriate for political discussion is excluded from philosophical

\footnotetext{
${ }^{97}$ Dworkin, op cit, above note $85, \mathrm{p} 49$.

${ }^{98}$ Ackerman, op cit, above note 65, p359.

${ }^{99}$ Neal, op cit, above note 84 , p666.
} 
discussion from the beginning. If this is the case then Neal is correct in calling the process coercive.

There is more to Ackerman's approach than this simple result. He goes on to criticise those who believe that liberalism is based on a grand metaphysical or epistemological error, and that the only response to this is to hack at its roots and branches. This approach, Ackerman claims, is misconceived. ${ }^{100}$

Liberalism does not depend on the truth of any single metaphysical or epistemological system. Instead, liberalism's ultimate justification is to be found in its strategic location in a web of talk that converges upon it from every direction. Each strand is itself sufficient to support a reasoned belief in Neutrality; yet to cut oneself off from a single strand hardly liberates from the web of belief. ${ }^{101}$

He goes on to say that

I do not, in short, claim that you can reason your way to Neutrality regardless of the way you understand your place in the world. Instead of proclaiming liberalism's complete independence from other branches of reflective talk, I mean to assert its relative autonomy. In order to accept liberalism, you need not take a position upon a host of Big Questions of a highly controversial character. So long as you understand your relation to the world in one of a number of familiar - if very different - ways, you will find it sensible to regulate our power struggle by means of Neutral dialogue. ${ }^{102}$

These passages move away from the idea of a close parallelism between political and philosophical dialogue, and from the idea that this philosophical dialogue prohibits non-liberal discussion. Instead, they suggest that determining neutrality as the best condition of a political dialogue does not imply any particular metaphysical or epistemic approach; that is not to say that it is independent of other knowledge, or of our personal contexts as human beings, but, rather, that the result can be achieved without any particular standpoint or approach in any of a number of different ways.

This conception is markedly different from those of Rawls, Nozick and Dworkin, who each search for a single justification of their liberal structures, based around the right over the good, natural rights and resource equality respectively. Ackerman suggests, instead, that neutrality is important for a number of different, cumulative and mutually exclusive reasons. In addition, these reasons do not depend on a carefully researched and understood philosophy, but, rather, on some simple results from thinking about

\footnotetext{
${ }^{100}$ Ackerman, op cit, above note 65, pp360-361.

${ }^{101}$ Ibid, p361.

102 Ibid.
} 
our moral and political lives: that people tend to use power in a way that is mistaken or corrupt; that no-one seems to know clearly the best way to live; that each person has a special moral standing; and that there may be no meaning to moral judgment anyway.

This is different not only in being a looser approach to moral philosophy, but also because it represents a response to moral uncertainty apart from those of Rawls, Dworkin and Nozick. This is the case of two of the paths to liberalism: uncertainty as to the best conception of the good, and moral scepticism. This is a reversal of the earlier approach that changes the very moral nature of liberal theory.

Ackerman's theory can be seen as an attempt at a new approach to justifying liberalism that embraces plural, alternative and potentially complementary justifications, and that implies a conception of moral theory that is sympathetic to moral uncertainty. There remain, however, a number of questions about this approach. Despite Ackerman's clarification, it seems like his philosophical dialogue is constrained so as to remove all arguments except for those that result in neutrality. In fact, it is quite consciously directed as justifying only political neutrality. What Ackerman fails to address is whether there are other political approaches that might as easily find their own paths as significant as those leading to neutrality, and also whether his four paths might just as easily lead elsewhere. Moral scepticism, for example, might lend itself more readily to a political free-for-all power game. A consequence of conceiving of philosophical theory as plural and open is that an anticipated result might not be unique in deserving consideration.

In addition, Ackerman's approach has been criticised on the grounds that it does not succeed in bypassing the Big Questions of philosophy, but, rather, implies a particular pronouncement on these questions. ${ }^{103}$ His image of philosophical dialogue may be plural and open, and may encourage some basic agreement among people, but this itself is an image that denies approaches that suggest that there is a single, best approach to moral philosophy, and those that suggest that his approach is inferior. This meta-ethical perspective is one that needs to be addressed in any attempt to

${ }^{103}$ Thigpin \& Downing, op cit, above note 83, p597. 
found a theory of liberalism on ideas that exclude or downplay the role of moral philosophy.

Overall, Ackerman's approach to justifying liberalism, as set out in Social Justice in the Liberal State, is important and thought-provoking; however, it suffers from a lack of detail. Fortunately, these ideas are taken up in different forms by various liberal theorists, as will be seen in subsequent chapters. In terms of substance, the justification of neutrality as the central liberal concern, and the derivation of equality from this neutrality, are both weak. The theory does contain, however, a number of important aspects, including the use of actual dialogue as a liberal device and the idea of an undominated genetic diversity. But, for present purposes, the shift in justification away from the idea of liberalism as an answer to moral uncertainty, and towards liberalism as a response to moral uncertainty, is most significant.

\section{Contemporary liberal theory}

The works of Rawls, Nozick, Dworkin and Ackerman, published between 1971 and 1981, constitute the early era of contemporary liberal theory. Each devises hypothetical situations that pare away irrelevant and arbitrary details and that, therefore, inform a conception of justice. Each involves interplay between various conceptions of liberal ideals, primarily between liberty, equality and neutrality. This interplay is expressed in two ways: for Rawls and Nozick as a moral priority - the right over the good and natural rights respectively; and, for Dworkin and Ackerman, the elevation of a conception of one of these ideals so that it becomes the central liberal concern - equality (of resources) for Dworkin and neutral (dialogue) for Ackerman. Nozick probably stands out from the others the most in using a system derived from a set of presupposed rights, which results in the least egalitarian system.

Each of these four recognisably liberal theories describes a particular space within which people are free to make their own choices and pursue their own conceptions of the good. This space is defined by the liberties, neutrality and equality. Liberties offer freedoms of action: neutrality prevents interference with action on grounds that 
discriminate between conceptions of the good; and equality implies some measure of equal opportunity to pursue a conception of the good. The interplay between particular conceptions of these ideals within a liberal theory specifies the nature and constraints of the space within which individuals are free to act.

This space reflects the openness of liberal theory. It is in eschewing the idea of a single privileged conception of the good, and committing, instead, to allowing individuals to determine their goals and actions that liberalism lays claim to the best expression of social justice. In this way, liberalism is an open system that allows individuals to make decisions about how they want to live. These decisions, however, must conform to the system of liberties itself. No liberal theory is perfectly open: none addresses and indulges the desires and conceptions of every citizen to the point of a free-for-all. Instead, there is a closedness to each liberal system, implicit in any set of liberties, that is defined by the restrictions on action and resource allocation. It is tempting for liberal theorists to believe that, because liberalism is essentially open to alternative ideas and ways of life, it does not need to defend itself against systems that suggest these alternatives; or, to believe that liberalism transcends less obviously open systems. But, the closedness implicit in any liberal conception of justice refers to particular positive ideas and conceptions that must be justified as being morally valuable. It is not enough for liberalism simply to claim value by virtue of its openness, without addressing the particular and closed ways in which this openness is conceived and defined.

The early works of contemporary liberal theory each take a different approach to justifying the substance of their systems. Rawls' is the most complete and is probably the most innovative. His ideas, of the reflective equilibrium and the use of our considered intuitions, are linked closely to the original position in order to justify his two principles of justice. Nozick is quieter on matters of justification and relies wholly on his set of natural rights. Dworkin relies on equality in a similar way, and the justification of his luck egalitarian approach to resource-equality depends on recognising the system itself as liberal and just. Finally, Ackerman's work represents a shift to incorporate some aspects of moral uncertainty into his justification, which he bases on the idea of a philosophical dialogue. 
All of these theories have in common a theoretical structure that implies their liberal approach to be, in some sense, universally valid. Despite differences in outcomes, structures and justifications, there seems to be justificatory errors common to all four: that is, the common reliance on conceptions or intuitions that are quite plausible, but, upon reflection, are revealed to be rationally and reasonably contestable. This is particularly problematic given that the readers of the theories are likely to be predisposed to accept liberal ideals.

This has the most pronounced effect when the contestable conception or ideal is raised to the status of absolute. Each of the theories seems to contain such an ideal, of the right over the good, natural rights, equal concern and respect, and dialogical neutrality. These conceptions are important in that they focus the theory and define the interaction of the various liberal conceptions, and they lend to the theory the promise of a deontological universality. Yet, each of the theories have been validly criticised on the grounds that, at best, these conceptions have not been adequately defined and justified as central; and, at worst, they reveal a bias in the closedness of each liberal theory. In relation to these theories, William Galston comments that

[w]e can discern a recurrent pattern. Each of these contemporary liberal theories begins by promising to do without a substantive theory of the good; each ends by betraying that promise. All of them covertly rely on the same triadic theory of the good, which assumes the worth of human existence, the worth of human purposiveness and the fulfilment of human purposes, and the worth of rationality as the chief constraint on social principles and social actions.... The liberal theory of the good is the theory of rationalist humanism. ${ }^{104}$

He identifies those implicit assumptions and ideals that support and inform particular liberal theories. Instead of justificatorily open to and neutral between various ways of life, Galston reveals liberal theory to apply particular, albeit general, conceptions, consistent with the ideals of rationalist humanism. In this way, the justifications of liberal theory fail in the way they purport to be general and open, and, instead, represent particular moral and political positions.

The justificatory challenge of this form of liberal theory is to create a system that is founded on a conception of justice and of our selves, and that defines a morally substantial liberal space within which people are free to determine their own

\footnotetext{
${ }^{104}$ W A Galston, 'Defending Liberalism' (1982) 76(3) American Political Science Review 621, pp625626.
} 
conception of how to live and act. The difficulty is that the conceptions and rules on which such a system relies are themselves contestable in a way that tethers it to a particular set of beliefs, and that denies the universal application and value of the theory. This is exacerbated by the sheer complexity of our moral lives, and of the plurality of beliefs that theorists attempt to confine as the subject of liberal freedom. Overall, these aspects of moral uncertainty, complexity and contestability have not been effectively overcome in these liberal theories. 


\section{PLURALISM}

\section{The communitarian critique of liberalism}

Those theories examined in the preceding chapters dominated liberal and political thought for the decade following the publication of A Theory of Justice. Despite various differences, the four had a roughly similar attitude to moral political-theory: a focus on the value and autonomy of the individual; a use of hypothetical situations to elucidate issues of justice; and, the use of complicated structures that define conceptions of equality, liberty and neutrality, and the relationships between them. The first major challenge to this approach came from a group labelled the communitarians. Their criticisms prompted a set of debates between communitarians and liberal theorists that were central to liberalism in the 1980s. ${ }^{1}$

The grouping of theorists and critics of liberalism into communitarians, as with any grouping of independent thinkers, is imperfect: it includes probably four or five people with diverse approaches and attitudes to moral theory, and who would not necessarily identify themselves as being part of any group. ${ }^{2}$ Nevertheless, they do represent a common criticism of liberal theory, the central claim of which has been described as "the necessity of attending to community alongside, if not prior to, liberty and equality". ${ }^{3}$ In this way, they each criticise the liberal approach that focuses on conceptions of liberty, equality and neutrality, and ascribe only secondary meaning to "the shared practices and understandings within each society."4

\subsection{The unencumbered self}

\footnotetext{
${ }^{1}$ S Mulhall \& A Swift, Liberals and Communitarians (1996), pxii.

${ }^{2}$ Ibid, ppxiv-xv.

${ }^{3}$ W Kymlicka, Contemporary Political Philosophy (2 ${ }^{\text {nd }}$ edn., 2002), p208.

${ }^{4}$ Ibid, p209.
} 
Michael Sandel's criticism of the conception of the person implicit in A Theory of Justice can now be viewed in the context of the liberal-communitarian debate. Sandel's Liberalism and the Limits of Justice, published in 1982, was the first major communitarian work, and is a critical reading of A Theory of Justice. One of Sandel's central themes is that Rawls' theory implies a conception of the person, not as having some particular and essential desire or drive, but with respect to the "constitutive understanding we have of ourselves". 5

Sandel's claim, then, is that Rawls' theory depends implicitly on a conception of the essential structure of our selves. This self is antecedently individuated; its essence lies in its ability to choose its own conception of the good, and its own ends. ${ }^{6}$

Where the self is disempowered because detached from its ends, dispossession is repaired by the faculty of agency in its voluntarist sense, in which the self is related to its ends as a willing subject to the objects of choice. The relevant agency involves the exercise of will, for it is the will that is able to transcend the space between the subject and its object without requiring that it be closed. ${ }^{7}$

In this way the Rawlsian self is antecedently unencumbered by its ends, and has the innate capacity to choose its own conception of what is good and how best to live. This picture of the self pervades Rawls' conception of the meaning of justice. The background of justice, in his words, consists in "the plurality of distinct persons with separate systems of ends". ${ }^{8}$ Justice defines the relationships between these individuals, in their vying for the fulfilment of their own particular ends, a conception that Sandel argues promotes plurality at the expense of unity. ${ }^{9}$

Generally speaking, it is the distance between the self and its ends that marks Sandel's concern with Rawls' conception of the person.

One consequence of this distance is to put the self beyond the reach of experience, to make it invulnerable, to fix its identity once and for all. No commitment could grip me so deeply that I could not understand myself without it. No transformation of life purposes and plans could be so unsettling as to disrupt the contours of my identity. No project could be so essential that turning away from it would call into question the person I am. ${ }^{10}$

\footnotetext{
${ }^{5}$ M J Sandel, Liberalism and the Limits of Justice ( $2^{\text {nd }}$ edn., 1998), p48.

${ }^{6}$ Ibid, p51.

${ }^{7}$ Ibid, p58.

${ }^{8}$ J Rawls, A Theory of Justice (revised edn., 1999), p25.

${ }^{9}$ Sandel, op cit, above note 5 , p53.

${ }^{10}$ Ibid, p62.
} 
The distance between self and ends shapes the original position, and, so, the two principles of justice. ${ }^{11}$ One's shared concerns and values, and one's sense of community, are subject to this same distance, and, so, are always peripheral to the self; these are chosen voluntarily from among alternatives, and do not define or constitute the meaning or essence of the self. As the self is prior to its ends, so a society is prior to any of the aims or shared values any of its members might possess. $^{12}$

The charge against Rawls, then, is one of circularity and liberal bias: that his conception of the self forms part of the foundation of his theory and, so, must itself be justified in order for its conclusions to be valid, otherwise its liberal conclusions are reached only because the theory's structure itself is liberal. The argument that a liberal theory conceives of the self as individual, pre-social and unencumbered by a sense of community is a typical communitarian criticism.

Another communitarian thinker, Charles Taylor, makes a similar argument about Rawls' implicit conception of the self, although his focus is more on how the self is actually constituted. Taylor, in Sources of the Self: The making of the modern identity, attempts to set out in detail the history and the moral sources of the modern, subjective identity. This identity, he argues, searches for meaning through selfexpression and self-interpretation. ${ }^{13}$ Moral judgments are made only in relation to interpretive frameworks. These distinguish between and give meaning to the various moral ethics that we might employ. ${ }^{14}$ These act as the background, or the horizons, for our moral judgments and intuitions, and are inescapable parts of our moral selves.

[T] he claim is that living within such strongly qualified horizons is constitutive of human agency, that stepping outside these limits would be tantamount to stepping outside what we would recognize as integral, that is, undamaged human personhood.... My identity is defined by the commitments and identifications which provide the frame or horizon within which I can try to determine from case to case what is good, or valuable, or what ought to be done, or what I endorse or oppose. In other words, it is the horizon within which I am capable of taking a stand. ${ }^{15}$

\footnotetext{
${ }^{11}$ Ibid, p49.

${ }^{12}$ Ibid, p64.

${ }_{13}^{13}$ C Taylor, Sources of the Self: The making of the modern identity (1989), p18.

${ }_{15}^{14}$ Ibid, pp25-26.

${ }^{15}$ Ibid, p27.
} 
This means that our individual moral selves have meaning only in relation to our interpretive frameworks. These, Taylor argues, are expressed in terms of language, and, so, our selves are inextricably linked to the linguistic communities of which we are part. In this way, our self-understanding and self-definition is dependant on our linguistic and social context. ${ }^{16}$ It is this sense that the Rawlsian conception of the person, as antecedently individuated, is incoherent, and amounts to a denial of the actual foundations of our selves. ${ }^{17}$

If the Rawlsian conception of the self cannot be sustained, then any liberal project of the Rawlsian type is undermined. Such theories rely on the unencumbered self as an essential element: an autonomous individual who makes valuable decisions as to their own conceptions of the good and their own ends; a political unit that is declared to have independent moral worth, and who should be respected.

The obvious response to this criticism is to assert that it is the individual that is the appropriate unit of moral and political concern, and, further, that liberal theories do not prevent individuals from acting in communal ways, nor from valuing communal beliefs. On the contrary, they permit people free association with whatever group and whatever corresponding conception of the good they choose. The point of Sandel, and implicitly of Taylor, is that, while this communal behaviour is permitted as the legitimate expression of one's conception of the good, the Rawlsian self has a more fundamental role in liberal theory. In relation to A Theory, for example, this conception informs the priority of the right over the good, as well as rational behaviour in the original position. That the outcome of such theories allows communal behaviour does not amount to adequate recognition of the idea that they are themselves built on an impoverished, and potentially incoherent, conception of the self.

This communitarian argument appears to have substantial merit, and certainly conveys a conception of the person that has more depth and more detail. Individual rationality and the personal autonomy to choose one's own goals, however, still retain considerable moral force. While it could not be denied that we all operate in front of a

\footnotetext{
${ }^{16}$ Ibid, pp35-36.

${ }^{17}$ See Mullhall \& Swift, op cit, above note 1, p112.
} 
horizon that is communally defined, it might still be argued that it is the individual who is the proper focus of liberal theory. If this argument is to be successful, however, the liberal must explain how the resulting conception forms a legitimate part of their theoretical structure, and how it coheres with the seemingly meaningful portrayal of the self offered by the communitarians.

What this communitarian criticism achieves, then, is to reveal a supposedly accurate and objective aspect of some early contemporary liberal theories, to contain liberal bias. This indicates a structural bias in liberal theory, as well as a potentially impoverished foundation. This requires that liberal theory restructure itself, by either incorporating a more communally-focussed conception of the self, or by explaining why the antecedently individuated self is a morally relevant point of focus.

\subsection{The enlightenment critique}

The communitarian critique of the unencumbered self is one of structure: that liberal theories tend to be built around an implicit conception of the person that is itself essentially liberal. Some communitarian arguments are more general, and attack the justificatory approach and meta-ethical attitudes of liberal theories.

Alasdair MacIntyre joins Sandel and Taylor in criticising the implicit conception of the person employed by liberal theorists, but, for him, this is merely one aspect of an approach to moral theory that is bankrupt. ${ }^{18}$ At the beginning of his book After Virtue, he describes an imaginary world where scientific knowledge has been previously lost, and an attempt is made to piece together and continue its work. ${ }^{19}$ This state of affairs, asserts MacIntyre, characterises contemporary moral theory:

The hypothesis which I wish to advance is that in the actual world which we inhabit the language of morality is in the same state of grave disorder as the language of natural science in the imaginary world which I described. What we possess, if this view is true, are the fragments of a conceptual scheme, parts which now lack those contexts from which their significance derived. We possess indeed a simulacra of morality, we continue to use many of

\footnotetext{
${ }^{18}$ Ibid, p71.

${ }^{19}$ A C MacIntyre, After Virtue (2 ${ }^{\text {nd }}$ edn., 1984), pp1-2.
} 
the key expressions. But we have - very largely, if not entirely - lost our comprehension, both theoretical and practical, of morality. ${ }^{20}$

MacIntyre identifies the beginnings of this loss as being the moral projects of the enlightenment of the eighteenth century. Social anthropologist Earnest Gellner has characterised the enlightenment as being

\begin{abstract}
concerned with describing, and above all denouncing, the society it was rejecting and which it hoped was passing for ever - a society based on tyranny and superstition. The rule of kings and priests was to give way to the rule of Reason and Nature. If falsehood had endorsed, legitimated and underwritten oppression and expectation, would not luminous truth engender a new, free and happy social order? It seemed a plausible hope, and the philosophes were dedicated to its propagation. ${ }^{21}$
\end{abstract}

The enlightenment involved the doubting of religion as the basis for an understanding of right action, and sought a shared, publicly justified, rational account of morality. ${ }^{22}$ This project, MacIntyre argues, failed completely. The main reason he offers for this is that the philosophers of the time "shared characteristics deriving from their highly specific shared historical background". ${ }^{23}$ It was this common scheme of moral beliefs that appeared as the true foundations of a rationally justified morality.

The realisation of this failure had, MacIntyre argues, a number of important effects, not the least of which was the common doubt in any shared moral rationale. ${ }^{24}$ It led also to what MacIntyre calls 'emotivism' in moral dialogue and theory. This emotivism is at the heart of the failure MacIntyre perceives in current moral theory. Emotivism is encapsulated by the idea that moral judgments are "nothing but expressions of preference, expressions of attitude or feeling". ${ }^{25}$ Thus, there is no objective or true moral judgment, simply the personal evaluations of individuals within the moral sphere. This means that there can be no rational justification of any supposedly objective or universal moral belief. ${ }^{26}$ MacIntyre argues that this emotivism, in various guises, forms a substantial part of post-enlightenment moral philosophy. ${ }^{27}$

\footnotetext{
${ }^{20}$ Ibid, p2.

${ }^{21}$ E Gellner, Conditions of Liberty: Civil Society and its Rivals (1994), p93.

${ }^{22}$ MacIntyre, op cit, above note 19, pp39, 50.

${ }^{23}$ Ibid, p51.

${ }^{24}$ Ibid, p50.

${ }^{25}$ Ibid, p12.

${ }^{26}$ Ibid, p19.

${ }^{27}$ Ibid, pp20-22.
} 
MacIntyre compares the distributive conclusions of Rawls and Nozick. He concludes that neither of the two impugns the approach of the other; in the sense that each operates independently of the other, that is, behind an imagined veil of ignorance, and with a set of entitled rights. ${ }^{28}$ MacIntyre seems to be saying that each occupies a different justificatory space, so that one does not make a direct statement against the other; the two results are incompatible, but they are not derived from arguments that are directly contrary to each other. This he describes as their incommensurability, which suggests that they are unable to be compared by any independent or common standard. $^{29}$

This is virtually all MacIntyre has to say on the subject of contemporary liberalism. Yet, his conception of enlightenment and emotivist influences in moral philosophy can be applied meaningfully to these theories. The obvious first step is to identify the ways in which emotivism is present. The autonomous, emotivist self resembles the antecedently individuated self implied by Rawlsian liberalism. In addition, the individualism implicit in the unencumbered self, and in the neutrality of liberalism between various conceptions of the good, resembles the emotivist attitude to moral judgment: that there is no rational superiority of one conception over another, and that each merely reflects the autonomous choice of each individual.

The difference is that this emotivism extends only to the good; to various conceptions of how it is best to live. Rawls and the other theorists search for universal justifications of rational liberal structures, in a way that reflects enlightenment aims. But these are not, with the possible exception of Nozick, expressed in ways that are congruent with the enlightenment way, as logical structures derived from universal conceptions. Instead, they incorporate variation in the conceptions of the good that people can hold.

This suggests a tension in contemporary liberal theory between the underpinnings of emotivism, implied by the autonomous individual and neutrality between conceptions of the good, and emotivism's enlightenment aims to achieve a universal rational moral theory or to overcome the separateness of moral theories in the justificatory

\footnotetext{
${ }^{28}$ Ibid, p249.

${ }^{29}$ Ibid.
} 
space. It makes sense, then, that Rawls and Ackerman employ novel justificatory strategies in order to reconcile the two. It also makes sense that the liberal theories must face the presumptions and biases that MacIntyre ascribes to enlightenment moral theories.

In his later book Whose Justice? Which Rationality? (1988), MacIntyre considers liberalism as a historical movement. He defines a 'tradition of enquiry' as being "such a movement in the course of which those engaging in that movement become aware of it and its direction and in self-aware fashion attempt to engage in its debates and carry enquiries forward". ${ }^{30}$ The overt aim of liberal thought, MacIntyre argues, is to transcend these traditions, yet the liberal movement itself has become such a tradition.

[T] he project of founding a form of social order in which individuals could emancipate themselves from the contingency and particularity of tradition by appealing to genuinely universal, tradition-independent norms was and is ... the project of modern liberal, individualist society, and the most cogent reasons that we have for believing that the hope of the tradition-independent rational universality is an illusion derived from the history of that project. For in the course of that history liberalism, which began as an appeal to alleged principles of shared rationality against what was felt to be the tyranny of tradition, has itself been transformed into a tradition whose continuities are partly defined by the interminability of the debate over such principles. ${ }^{31}$

MacIntyre focuses on the universality of liberalism's aims, and on its failure to meet these aims. This failure is in not recognising its own nature as a tradition, socially and philosophically located.

MacIntyre goes on to characterise two central problems of liberalism: the liberal self and the common good. He claims that a self is implied that represents a falsely unified, well-ordered will, and that this implies the single overarching good of liberalism itself. ${ }^{32}$ These arguments are both familiar: the former is dealt with in the preceding section, and the latter emphasises liberalism as a closed system that must justify itself as such. What is interesting here is that these criticisms are presented in a social context, as part of a critique of historically informed attitudes to moral theory.

Philosopher John Gray has made these arguments more explicit in declaring that liberalism has failed in its attempt to attach itself to universal foundations.

\footnotetext{
${ }^{30}$ A C MacIntyre, Whose Justice? Which Rationality? (1988), p326.

${ }^{31}$ Ibid, p335.

${ }^{32}$ Ibid, pp346-347.
} 
It is characteristic, and perhaps definite, of liberalism that it should seek to ground the historical contingencies of liberal practice in a foundation of universally valid principles.... Liberalism, which in its applications to personal conduct aims for toleration, and even pluralism, is in its political demands an expression of intolerance, since it denies the evident truth that many very different forms of government may, each in its own way, contribute to an authentic mode of human well-being.... Because of its universalizing doctrinal zeal, liberal thought has always sought to elevate liberal practice into a set of principles, and then to demonstrate the unique claim on reason of those principles. ${ }^{33}$

This argument is a clear expression of the ideas suggested by MacIntyre. It includes an explicit acceptance of the closedness of liberal systems, and accuses liberalism squarely of making false claims of its moral power. This implicitly denies the neutrality of liberalism, a point made more plainly by Gray in a later work when he states that it is arguable that the scholarship borne out of A Theory of Justice "has done little more than articulate the prejudices of an Anglo-American academic class that lacks any understanding of political life in our age". ${ }^{34}$ This claim is more brash than any made by MacIntyre, but the underlying idea is similar; liberal theory is located socially, and the sources of its bias are identified.

The two communitarian criticisms are both important to liberalism. The first sets out the idea that a conception of self as unencumbered forms an implicit and contestable part of each of these theories. The second accuses liberalism of seeking a rational justification appropriate to a universal theory, and of failing to conceive of itself as the product of a contingent social and political context. Both of these suggest that the early contemporary liberal theories, in both justification and substance, contain a liberal bias that undermines any assertions of universality. As such, they repeat and illustrate many of the arguments discussed in previous chapters. In particular, the more general criticism emphasises the failure of liberalism to achieve its universal aims.

MacIntyre's characterisation of emotivism and enlightenment universalism together offers an interesting perspective on liberalism: as aiming at a constructed universal moral theory, and, also, admitting the plurality of personal beliefs that are rationally insoluble. Rawls' resolution of this tension, to base the former on the right and restrict the latter to the good, has already been doubted. Overall, these communitarian

\footnotetext{
${ }^{33}$ J Gray, Liberalisms (1989), p239.

${ }^{34} \mathrm{~J}$ Gray, Enlightenment's Wake: Politics and culture at the close of the modern age (1995), p1.
} 
criticisms present a powerful and fundamental challenge to liberal theory, and in particular raise the issue of whether and how any moral analysis can overcome the bias implied by the social location of its practitioners.

\section{Liberal responses}

\subsection{Pluralism in liberal theory}

Because communitarianism played so central a role in the development of liberal theory, a positive liberal response to communitarianism was required. This came in a number of forms: both from Rawls himself, and in the work of other liberal theorists. That the communitarian criticism broke down the Rawlsian approach to theory, gave theorists the impetus to shift the focus and approach of liberalism. One of the general and important aspects of the post-communitarian shift was the holding of difference to be the central concern of liberal theory. The most common expression of this is the increasing role of a conception of pluralism as part of liberalism.

The idea of pluralism conveys the essential multiplicity or diversity of its subject matter. All of contemporary liberal theory recognises pluralism in one way or another. A Theory of Justice, for example, works from the premise of a plurality of individual conceptions of the good. Often, pluralism featured as a morally disequalising fact of the world to be overcome or corrected for by liberalism. After the communitarian criticisms, pluralism took on a new prominence in the structure of liberal theories, and, ultimately, played the role of the central justificatory concern, superseding equality or neutrality.

This pluralism often took a form similar to that described by Isaiah Berlin. Berlin's essay, 'Two Concepts of Liberty', originally presented in 1958, is probably the most significant work of political theory prior to A Theory. Its main task is to set out two conceptions of freedom: positive freedom, which means the desire to take control of one's life and to be one's own master; and negative freedom, which is the absence of 
interference with one's body or activity. ${ }^{35}$ Berlin ultimately prefers the latter conception to the former in that it involves less coercion, and, so, is the safer form of liberty. ${ }^{36}$

Berlin also asserts a pluralism of irreducible and potentially conflicting values. ${ }^{37}$ This means that it is not possible for any single human project or system to satisfy all of our values, and that conflict, and even tragedy, is the inevitable outcome of any such attempt. For this reason, Berlin argues, it is better for a state to be tolerant of different values than to risk authoritarian coercion by imposing positive ideals of freedom.

\begin{abstract}
Pluralism, with the measure of 'negative' liberty that it entails, seems to me a truer and more humane ideal than the goals of those who seek in the great, disciplined, authoritarian structures the idea of 'positive' self-mastery by classes, or peoples, or the whole of mankind. It is truer, because it does, at least, recognise the fact that human goals are many, not all of them commensurable, and in perpetual rivalry with one another.... It is more humane because it does not (as the system-builders do) deprive men, in the name of some remote, or incoherent, ideal, of much that they have found to be indispensable to their life as unpredictably self-transforming human beings. ${ }^{38}$
\end{abstract}

In this way, Berlin sees the pluralism of values as being at the centre of his reasoning for negative liberty and toleration. By contrast, Rawls relegates differences resembling these to the domain of conceptions of the good, which he subordinates to matters of the right. The communitarian arguments against his general approach led to liberalism granting this pluralism a more central and justificatory role in theory. This will be explored by looking at the theories of Walzer, Raz and Gray, and, then, by considering in general terms the relationship between pluralism and liberalism.

\title{
2.2 Walzer's complex equality
}

Michael Walzer is more readily identified as a communitarian than a liberal, yet, in his Spheres of Justice: A Defence of Pluralism and Equality (1983) he offers a positive and recognisably liberal theory that expresses the idea that justice is not absolute or universal, but is relative to social meanings. ${ }^{39}$

\footnotetext{
${ }^{35}$ I Berlin, The Proper Study of Mankind: An Anthology of Essays (1998), pp194, 203.

${ }^{36}$ Ibid, pp234-237, 241.

${ }^{37}$ Ibid, p239.

${ }^{38}$ Ibid, pp241-242.

${ }^{39}$ M Walzer, Spheres of Justice: A Defence of Pluralism and Equality (1983), p312.
} 
Walzer's theory involves the use of two conceptions, social goods and spheres of justice, tied together by the necessity of a complex equality. He argues that the natural philosophical impulse in searching for a valid form of equality is to look for some underlying unity: "a short list of basic goods, quickly abstracted to a single good; a single distributive criterion or an interconnected set". ${ }^{40}$ This simple equality, he argues, is a misunderstanding of distributive justice, as it ignores its potentially plural nature, and claims, instead, that "it is doubtful that it [justice] can be made in only one way". 41

The subjects of this distributive justice are goods, conceived broadly and given meaning through their social context. All goods, he argues, are social goods; no good has meaning independent of the society and culture that values it. ${ }^{42}$ These goods are not only used by us, but our relationship to them informs our identities. ${ }^{43}$ The plural and contextual nature of goods dictates the nature of a complex equality, and directs the use of multiple principles of justice.

\footnotetext{
The questions posed by the theory of distributive justice admit of a range of answers, and there is room within the range for cultural diversity and political choice. It's not only a matter of implementing some singular principles or set of principles in different historical settings. No one would deny that there is a range of morally permissible implementations. I want to argue more than this: that the principles of justice are themselves pluralistic in form; that different social goods ought to be distributed for different reasons, in accordance with different procedures, by different agents; and each of these differences derive from different understandings of the social goods themselves - the inevitable product of historical and cultural particularism. ${ }^{44}$
}

From the foundational premises that goods are the subject of justice, and are plural and their meaning contextual, Walzer concludes that justice itself must be plural and contextual, and can be conceived only in relation to the society that it affects.

This brings us to the notion of what justice, or complex equality, actually entails. Walzer identifies it as the absence of injustice, the latter being characterised by dominance and monopoly in the distribution of goods:

\footnotetext{
${ }^{40}$ Ibid, p4.

${ }^{41}$ Ibid, p5.

${ }^{42}$ Ibid, p7.

${ }^{43}$ Ibid, p6.

${ }^{44}$ Ibid, pp5-6.
} 
Dominance describes a way of using social goods that isn't limited by their intrinsic meanings or that shapes those meanings in its own image. Monopoly describes a way of owning or controlling social goods in order to exploit their dominance. ${ }^{45}$

Social goods, Walzer argues, each have their own spheres in which they operate "freely, spontaneously and legitimately". 46 The nature of equality involves preventing dominance, which means that social goods are allowed to operate only in their own spheres. When each of the various spheres of goods functions autonomously, free from the influence of the goods of other spheres, then dominance is avoided and justice is served. ${ }^{47}$ This leads Walzer to his principle of non-dominance:

No social good $x$ should be distributed to men and women who possess some other good $y$ merely because they possess $y$ and without regard to the meaning of $x^{48}$

The task of justice, then, is to define these spheres in relation to a particular society, and place boundaries between them. This is the subject of much of the remainder of Walzer's book; each chapter maps out a new sphere of public goods and marks off its territory from that of the other spheres. In this vein he looks at social membership, security and welfare, money and commodities, education, and political power, among other spheres. In relation to money and commodities, for example, Walzer identifies a number of illegitimate uses of money, or 'blocked exchanges'. These include engaging in the buying or selling of political power, marriage and procreation rights and political offices. ${ }^{49}$ They are blocked to prevent money from playing a role in other social spheres.

By applying the principle of non-dominance, Walzer reaches a set of rules of justice that are specific to a particular society, and to the meaning that society gives to its goods. This allows the actual principles to be complex and defined by the belief system in question. This, Walzer argues, is appropriate to justice since it operates in relation to people and meanings that are themselves complex, plural and contextual.

There are an infinite number of possible lives, shaped by an infinite number of possible cultures, religions, political arrangements, geographical conditions, and so on. A given society is just if its substantive life is lived in a certain way - that is in a way faithful to the shared understandings of the members. ${ }^{50}$

\footnotetext{
45 Ibid, pp10-11.

${ }^{46}$ Ibid, p19.

${ }^{47}$ Ibid, pp19-20.

${ }^{48}$ Ibid, p20.

${ }^{49}$ Ibid, pp100-101.

${ }^{50}$ Ibid, p313.
} 
Walzer finds justice in the faithfulness of the way people live to their shared understandings; this is interlaced with the idea that shared understanding will draw boundaries between spheres of goods, and prohibit a person gaining influence in one sphere merely because of their influence in another.

Walzer's general approach is refreshingly simple: it is derived from a conception of the nature of the subject of justice. It has the communitarian virtue of being based on social and shared meaning of goods, rather than being neutral between individual conceptions of the good, and, as a consequence, is explicitly contextual and particular. In this way, it seems to avoid the communitarian criticisms of liberalism's conception of the unencumbered self, and its reaching for universal justification. It also offers an answer to the difficulty inherent in finding a single conception of liberal equality: that such a project misunderstands the nature of goods, and that any such equality must be complex, that is, plural and contextual.

The substance of Walzer's theory is also simple: it maps the interplay between an understanding of the social meanings of goods, and the separation of their various spheres. This separation is a novel and ingenious approach to conceiving justice, and accords with many plausible ideas about the improper use of power. It does, however, imply a particular structure of goods, and that breaches of the boundaries set out in this structure are unjust.

Linda Mullenix describes this approach as resting on a "somewhat fanciful assumption that the world's social goods can be neatly divided into autonomous spheres". ${ }^{51}$ She describes Walzer's boundaries as "dressed up truisms" that simply repeat familiar ideas of justice. ${ }^{52}$ However, since these spheres reflect the existing social meaning of goods, such a criticism seems beside the point.

Govert Den Hartogh has taken Mullenix's argument one step further and asserts that there is no structural distinction between the spheres; no clear, rational conception of

\footnotetext{
${ }_{51}^{51}$ L S Mullenix, 'The Limits of "Complex Equality"' (1984) 97(4) Harvard Law Review, 1801, p1806.

${ }^{52}$ Ibid, pp1807-1808.
} 
where the boundaries should lie. ${ }^{53}$ He goes on to point out that, because of the complexity of the principles that attach to spheres, different goods within a sphere can be distributed according to different principles. A good that is treated differently might be part of a complex sphere, or might just as easily form a new autonomous sphere.

The concept of "sphere" should therefore be understood as nominalistically as possible. We may, for instance, choose to discuss "the sphere of education" or the separate spheres of "basic, liberal and professional education" as it pleases us.... Nothing depends on the decision; it is only a matter of convenience of exposition. ${ }^{54}$

Because Walzer has made the application of justice to spheres of goods potentially complex and plural, and because of the lack of clear distinction between spheres, it seems that the boundaries are neither obvious nor particularly meaningful; there seems no necessary difference between setting boundaries between a number of spheres, and having a single sphere where various principles of justice apply in various different ways to the diverse goods the sphere encompasses. Given these difficulties, it seems that Walzer's spheres and principle of non-dominance are valueless. It may be that it is wrong for power in one area to mean power in another; but, there is no clear structure of spheres, nor a clear delineation of the proper effect of distributive principles, that would allow his system to function.

What remains of Walzer's theory is the use of social meaning to inform the use of a potential plurality of principles. The use of actual social meaning is a particularistic and relativistic move. It has been criticised as involving looking essentially at what is, rather that what is just, and also for failing to correct for potentially unreasonable or ignorant beliefs. ${ }^{55}$ As has been argued by James O'Fallon:

Walzer's fragmented good-by-good, sphere-by-sphere analysis of shared social meaning does not preclude a replication of the society of apartheid. Depending on the social meaning that a society attaches to goods, blacks, Hispanics, or women might find themselves principle-byprinciple, sphere-by-sphere at the bottom of the heap, not meriting education or office, not deserving honour, not wanted in association. ${ }^{56}$

\footnotetext{
${ }^{53}$ G Den Hartogh, 'The Architectonic of Michael Walzer's Theory of Justice' (1999) 27(4) Political Theory 491, p496; R Arneson, ‘Against 'Complex' Equality' in D Miller \& M Walzer (eds), Pluralism, Justice and Equality (Oxford: Oxford University Press, 1995), p238.

${ }_{55}^{54}$ Den Hartogh, op cit, above note 53, p502.

${ }^{55}$ Mullenix, op cit, above note 51, p1810; Arneson, op cit, above note 53, p240.

${ }^{56}$ J M O'Fallon, 'The Meanings of Justice' (1983) 62 Texas Law Review 763, p772.
} 
Because Walzer relies so heavily on the social meanings of goods to define the distributive principles that should be applied, he leaves himself open to this sort of argument. He might respond that the social meaning that is being sought is the meaning of justice; of what principles should apply, rather than what principles actually do apply. This might give the theory a greater ability to be critical and dynamic, but, ultimately, Walzer cannot avoid the possibility that it may prescribe the perpetuation of a system that by today's standards is abhorrent.

The point of Walzer's theory, however, is to view the justice of a society by its own standards and meanings. It is not about abolishing slavery or apartheid; it is about finding an understanding of justice that is faithful to the beliefs of the people involved. In Walzer's words, “[t]here are no external or universal principles that can replace [social meaning]. Every substantive account of distributive justice is a local account." ${ }^{57}$ Such an account is critical and dynamic because these beliefs are critical and dynamic.

The only non-contextual, substantive content Walzer provides is as to the spheres of justice and the principle of non-dominance, but the value of these is highly doubtful. Without them, rather than a system, Walzer's theory seems to be more an abstract conception of justice as an expression of the shared understandings of those involved. But this leaves a number of questions unanswered. Walzer never explains, for example, how justice can be given substance out of social meaning, in other words, how we get from a common understanding of the subject of justice to a substantive and valid system of justice. The justice-content of an understanding of goods and how they relate to us is not readily discernible, and this is partly behind criticisms that social meaning focuses on what is and not what is just: social meaning may provide the backdrop for justice, but it is not justice itself, it is more what various goods mean to us.

The process of deriving justice from social meaning, and even of discerning what social meaning is in the first place, is difficult and vague. The possibility of using such a process simply to justify an existing power structure is very real; as is finding a

\footnotetext{
${ }^{57}$ Walzer, op cit, above note $39, \mathrm{p} 314$.
} 
justice that simply reflects the bias of the individual or group (the point is to find a justice that reflects the bias of the whole of society). Walzer offers that:

The appropriate arrangements in our own society are those, I think, of a decentralized democratic socialism; a strong welfare state run, in part at least, by local and amateur officials; a constrained market; an open and demystified civil service; independent public schools; the sharing of hard work and free time; the protection of religious and familial life; a system of public honouring free from considerations of rank or class; workers' control of companies and factories; a politics of parties, movements, meetings and public debate. ${ }^{58}$

This list is arranged in terms of bounded spheres of goods. Some items are obvious and scarcely need mentioning; others are contestable and contested. The latter cannot reasonably be called social meanings and may simply reflect Walzer's own beliefs.

Walzer's theory, then, is founded on the idea that justice is plural and contextual, as people and societies are plural and contextual. Taking into account the failure of the bounded spheres argument, little further is actually said about the substance of the general theory. This has led Den Hartogh to assert that the theory itself is "only a disguise", which hides "a very effective way of doing applied ethics without theory". ${ }^{59}$ This 'way' is to specify and balance principles of justice by looking at the social meanings of the corresponding goods, instead of searching for a universal set of principles. $^{60}$

Whether this approach can be practiced without theory is unclear, but it is clear that it is expressed by Walzer through theory. What characterises this use of the theory is a focus on complexity and pluralism at the substantive level, and particularism (rather than universalism) and relativism at the meta-ethical. Although Walzer recommends a socially-contextual approach, that does not mean he can be described simply as a relativist. As William Galston has recognised,

Walzer's relativism is actually quite constrained. To begin with, he deploys a non-contextual, universalistic, and thoroughly traditional conception of philosophic truth. His entire theory of justice is presented as a transcontextual metatheory, structurally valid for all communities... And the nerve of his relativism - the assertion that it is not possible to rank-order social worlds - is itself put forward, not as an interpretation of our existence, but as a universal truth. ${ }^{61}$

\footnotetext{
${ }^{58}$ Ibid, p318.

${ }^{59}$ Den Hartog, op cit, above note 53, p518.

${ }^{60}$ Ibid.

${ }^{61}$ W A Galston, 'Community, Democracy, Philosophy: The Political Thought of Michael Walzer' (1989) 17(1) Political Theory 119, pp122-123.
} 
He goes on to identify further universals in Walzer's theory of the respect for social meanings, and of people as culture-creating creatures. ${ }^{62}$ Like A Theory of Justice, Walzer's theory makes a number of universal claims, including a broad conception of the self and the moral value of respect, although both of these have a social rather than an individual focus. What is different between these theories of justice is the nature of the approach, and of the universality being asserted. Rawls himself incorporates a type of pluralism in his theory, but this is in relation to different conceptions of the good, and, so, the prescribed liberal response is to design institutions according to the right that do not discriminate between these conceptions.

For Walzer, the complex and plural content actually forms part of the substance of the theory; instead of trying to sidestep this plurality, it defines how a society ought to organise itself in order to be just. Instead of a set structure of distributive principles, he argues that we should adopt any of a number of various principles, depending on the context of the situation and the social understanding of the goods being distributed. This contextual approach represents an interesting step in liberal theory, but faces a number of important challenges. Walzer has failed to give an adequate justification for his approach beyond the assertion that human understanding is complex and plural. There is a distance between this observation and the conclusion that no single set of principles can consistently deliver justice, and that justice is to be found in our social understanding of the subject matter involved. Also, the method by which this meaning can be found and the way in which it can define justice should be explained further in order to counter the argument that Walzer's system simply reflects what is, and ignores what is just. The very fact of the plurality and complexity of meanings makes the idea of finding and agreeing on a just approach in a particular situation seem unrealistic.

\subsection{Raz's perfectionism}

\footnotetext{
${ }^{62}$ Ibid, pp123-124.
} 
Joseph Raz entered the liberal debate with the publication of The Morality of Freedom in 1986. Instead of being a theory of justice, this book is a far-reaching treatise on political morality, and includes a number of parts that are beyond the scope of this thesis. As a work of liberal theory, it is known for incorporating aspects of communitarianism, and as a critique of the anti-perfectionism and neutrality in earlier conceptions of liberal justice. ${ }^{63}$

Perfectionism is the term used by Rawls to describe a moral theory that aims at advancing a particular conception of the good, such as to some expression of excellence or culture. ${ }^{64}$ Rawls explicitly argues against this approach, and, instead, that liberal theories should advance the right, and be neutral between conceptions of the good.

Raz responds that this neutrality is an illusion. Firstly, there is an implicit liberal bias to this neutrality. ${ }^{65}$ The communitarian argument that liberalism favours the individual over communal values is one example of this. Secondly, this position of neutrality is dependant on a particular baseline, which is itself not neutral. ${ }^{66}$ There is some value to this argument: even in Ackerman's highly constrained and history-free spaceship narrative, the baseline is shaped by the fact that genetic effects already belong to their biological 'hosts'. This argument does not deny the value of neutrality; it simply identifies the implicit baseline and requires of liberal theory that this is independently justified - just as Ackerman attempts to justify the allocation of genetic entitlements by using the separate principle of genetic non-domination. These arguments together suggest that any conception of neutrality is necessarily complex and depends on the structural context of the theory in question.

Raz argues that the better liberal response is to eschew any nominal ideas of neutrality and anti-perfectionism, and to permit the subjugation of repugnant conceptions of the good. ${ }^{67}$ Instead of neutrality, Raz adopts autonomy as the central liberal concern. In order to describe the nature and requirements of this autonomy, a conception of the

\footnotetext{
${ }^{63}$ Mulhall \& Swift, op cit, above note 1, p309.

${ }^{64}$ Rawls, op cit, above note 8, p286.

${ }^{65}$ J Raz, The Morality of Freedom (1986), p1 19.

${ }^{66}$ Ibid, p121.

${ }^{67}$ Ibid, p417.
} 
self is required. In the absence of neutrality, Raz is able to unselfconsciously conceive of the autonomous person as being a goal-directed part-author of their own lives, whose well-being "consists in the successful pursuits of self-chosen goals and relationships" ${ }^{68}$ As such, autonomy requires the availability of adequate options, and a minimum number of coerced choices. ${ }^{69}$

This autonomy is constrained in two important ways. Firstly, Raz recognises that individuals do not author the whole of themselves; they are already situated within a social context that is the background and not solely the outcome of the exercise of autonomy. ${ }^{70}$ Secondly, as a consequence of his perfectionist stance, autonomy is constrained by the disapproval of immoral and repugnant ways of life. ${ }^{71}$ The latter, Raz argues, does not represent a significant effect on autonomy. ${ }^{72}$

Rawls defines perfectionism in a political theory as the championing of some particular conception of the best way to live. The question that arises in relation to Raz's theory is how perfectionism can coexist with autonomy; in other words, what prevents Raz's perfectionism from removing every less valuable form of life, until a single, 'best' conception of the good remains. The answer lies with Raz's use of pluralism:

\footnotetext{
Moral pluralism claims not merely that incompatible forms of life are morally acceptable, but that they display distinct virtues, each capable of being pursued for its own sake. If the active and contemplative lives are not merely incompatible but also display distinctive virtues then complete moral perfection is unattainable. Whichever form of life one is pursuing there are virtues which elude one because they are available only to people pursing alternative and incompatible forms of life. ${ }^{73}$
}

Because different and incompatible lives have distinct virtues, there is no single way of life that society ought to promote. His perfectionism, then, is limited by the fact that "there are several maximal forms of life", rather than just one. ${ }^{74}$ It subordinates only those ways of lives that are manifestly wrong.

\footnotetext{
${ }^{68}$ Ibid, p370.

${ }^{69}$ Ibid, pp371-373

${ }^{70}$ Ibid, p369.

${ }^{71}$ Ibid, p381.

72 Ibid.

${ }^{73}$ Ibid, p396.

${ }^{74}$ Ibid.
} 
Raz reinforces this view of pluralism by reference to two additional factors, namely the related ideas of social forms and incommensurability. Our well-being depends on pursuing comprehensive goals that must, he argues, be based in existing social forms. ${ }^{75}$ These are defined as "forms of behaviour which are in fact widely practiced in [a] society", and that define and determine a person's success in social pursuits. ${ }^{76}$ So, even though he promotes a conception of people as self-authoring, Raz recognises both that this authorship is partial, and that it takes place within the context of a society. Because goals and well-being are necessary to autonomy, it is itself dependant on social forms.

The second factor that supports Raz's pluralism is the incommensurability of our comprehensive goals. He states that:

$\mathrm{A}$ and $\mathrm{B}$ are incommensurable if it is neither true that one is better than the other nor true that they are of equal value. ${ }^{77}$

Raz also mentions the mark of incommensurability, which is where neither A nor B is better than the other, and this remains the case even when one is improved. ${ }^{78}$ Both of these situations involve the inability to compare the overall value of the two in a meaningful way. This incomparability might conceivably be explained in a number of ways: as uncertainty as to what criteria to use to compare two things; or, as uncertainty as to how to apply an agreed criterion. ${ }^{79}$

This incommensurability, however, is not an imperfection:

\begin{abstract}
The mistake in this thought is that it assumes that there is a true value behind the ranking of options, and that the ranking is a kind of technique for measuring this value. It is true of course that when we express a judgment about the value of options we strive to identify what is true independently of our valuation. But the ranking which determines the relative value of options is not a way of getting at some deeper truth, it constitutes the value of the options. Values may change, but such a change is not a discovery of deeper truth. It is simply a change in value. Therefore, where there is incommensurability it is the ultimate truth. There is nothing further behind it, nor is it a sign of an imperfection. ${ }^{80}$
\end{abstract}

\footnotetext{
${ }_{76}^{75}$ Ibid, pp308-309.

${ }^{76}$ Ibid.

${ }^{77} \mathrm{Ibid}, \mathrm{p} 322$.

${ }_{79}^{78}$ Ibid, pp325-326.

${ }^{79}$ Ibid, pp326-327.

${ }^{80} \mathrm{Ibid}, \mathrm{p} 327$.
} 
Although we may choose between activities, or between comprehensive goals to pursue, this does not indicate some deeper and true value. Incommensurability means then that, although we may prefer one option to another, this does not make one better than the other; the judgment, instead, is contextual, and is subject to being different between people, or with the same person at different times or in different situations.

That plural ways of life are incommensurable in this way, and their values dependant on contingent social forms, defines a strong value-pluralism. ${ }^{81}$ This pluralism allows Raz to champion the autonomous choice between any moral and non-repugnant ways of life, despite his explicit perfectionism. It leads him to prescribe broad ideas of toleration between individuals, and freedoms that are instrumental to the exercise of his autonomy thus constrained. ${ }^{82}$

The centre of Raz's liberal scheme is his conception of autonomy, informed on one side by an incommensurable pluralism, and on the other by perfectionism. This autonomy, to make good choices, must be justified in its value and its location as the primary conception of liberal concern. There is a basic tension in Raz's account of autonomy between its absolute value as the most important of all socially valuable conceptions, and its restriction in application to choices that are good. As Donald Regan points out:

there is no inconsistency in saying that autonomy is autonomy even when it chooses the bad, and saying also that autonomy is valuable only when it chooses the good. But there is an inconsistency in asserting both of these propositions and in supposing also (as Raz does) that autonomy tout court is valuable. These three propositions taken together entail that autonomy which chooses the bad both is and is not valuable. ${ }^{83}$

Raz needs to admit, either, that autonomy exercised badly is not autonomy, or that autonomy is not always valuable. The first of these would mean that the definition of autonomy depends on the moral value of the actual choice made. The second would subordinate autonomy to that value, and, so, place Raz's identification of autonomy as the central liberal concern in jeopardy. Both conflict with the general principles of that theory, and bring his conception of autonomy into question.

\footnotetext{
${ }^{81}$ Ibid, p398.

${ }^{82}$ Ibid, pp401-412.

${ }^{83}$ D H Regan, 'Authority and Value: Reflections on Raz's Morality of Freedom' (1988) 62 Southern California Law Review 995, p1084.
} 
Perhaps the better way of understanding Raz's account of autonomy is by looking to the nature of its justification. There has been some debate as to whether this autonomy is universally valuable or merely valuable to liberal societies. ${ }^{84}$ At times, The Morality of Freedom does seem to justify autonomy in a contextual way:

In western industrial societies a particular conception of individual well-being has acquired considerable popularity. It is the ideal of personal autonomy. It transcends the conceptual point that personal well-being is partly determined by success in willingly endorsed pursuits and holds the free choice of goals and relations as an essential ingredient of individual wellbeing. ${ }^{85}$

At other points in the book, Raz seems to endue autonomy with a special and universal quality. He begins by asking whether autonomy is only valuable if chosen, and decides that this idea is attractive but incorrect.

There are powerful reasons telling in favour of the view that personal autonomy is only one valuable style of life, valuable to those who choose it, but that those who reject it are none the worse for that. Their refutation is important to gain a proper understanding of the special features of autonomy which distinguish it from ordinary valuable options, such as playing golf, or becoming a nurse. ${ }^{86}$

Raz expands on this position in a later, explicit response to arguments about the contextuality of autonomy. He does state that, to be a universal value, it must be the case that "people who lack personal autonomy cannot be completely well-off, or have a completely good life." ${ }^{87}$ He then seems to suggest that societies where there is no personal autonomy can, in fact, have a high level of well-being. Raz states:

I do not see that the absence of choice diminishes the value of human relations or the display of excellence in technical skills, physical ability, spirit and enterprise, leadership, scholarship, creativity, or imaginativeness, which can all be encompassed in such lives. ${ }^{88}$

Raz does not clearly deny that autonomy has universal value, but, on balance, he seems to employ some sort of contextual argument for its justification. He suggests above that, in western societies, autonomy is essential to individual well-being, and that the individual cannot avoid or opt out of this. He also suggests that a citizen of a non-autonomous society can, nonetheless, enjoy a great measure of well-being. This seems to imply that his account of autonomy is relevant to those socialised in an

\footnotetext{
${ }^{84}$ E.g. D McCabe, 'Joseph Raz and the Contextual Argument for Liberal Perfectionism' (2001) 111 Ethics 493, p494.

${ }^{85}$ Raz, op cit, above note 65, p369; see also J Raz 'Facing Up: A Reply' (1988) 62 Southern California Law Review 1153, p1227.

${ }^{86}$ Raz, op cit, above note $65, \mathrm{p} 390$.

${ }^{87}$ Raz, op cit, above note 85, p1227.

${ }^{88}$ Ibid.
} 
autonomous environment. To these people, choice is a necessary aspect of the value of what is chosen. ${ }^{89}$

This is a significant step away from earlier universal liberal theories. Here, the conception of central concern is contingent on pre-existing liberal characteristics. In this way, Raz's theory, insofar as it sets the foundation for a liberal theory of justice, is an admittedly biased liberal document. Its value is asserted only for those from a certain tradition, and its project is only to structure pre-existing liberal attitudes to justice in a cogent and attractive way.

There are a number of challenges faced by this contextualised conception of liberalism. Perhaps the greatest is the strong account of autonomy suggested by Raz. He argues not only that it is essential to our personal well-being, but that it is essential in such a way that society's primary ethical concern is to best satisfy autonomy. The first of these is questionable; it might be argued, for example, that choice does not play the role that Raz suggests, and that we might be able to achieve some level of well-being without the extensive autonomy that we westerners now enjoy.

But the latter is a more serious claim, and means that, effectively, autonomy, or the availability of good options, is the highest political goal; higher than the substance of the options themselves. The absolute strength and priority of this assertion seems to belie the plurality and complexity that Raz attributes to our other political beliefs and understandings.

For such a strong and yet contextual theory of autonomy to succeed, three things would need to be achieved. Firstly, an extensive sociological account of autonomy would be required. In particular, Raz would need to explain why autonomy is the essential feature of liberal societies, and not of non-western societies, when both feature a significant degree of freedom, formed and constrained by political and social institutions and practices. Secondly, this account of autonomy would need to be related to a conception of morality and justice, in order to be justified as the proper focus of a liberal political system. In particular, this justification must explain the

\footnotetext{
${ }^{89}$ See ibid, p1228.
} 
subordination of other socially valued conceptions. Finally, the contextual account of autonomy must be set within a meta-ethical theory that justifies a contextual ethical approach. Raz must explain why this manner of justifying moral theory is appropriate, as opposed, say, to looking for some universal truth that may be less obviously a part of the actual substance of a particular society or culture.

Raz satisfies these requirements in only a superficial way. He offers a socialised account of autonomy and well-being that may be quite powerful, but that is examined only cursorily. The moral reason for pursuing autonomy so ardently is presumably to promote well-being within a liberal state, but this is not explored sufficiently, and neither is his meta-ethical approach. These shortcomings may be attributed to the fact that Raz's contextual account of autonomy is tentative and fleeting. It seems that he has not addressed fully the difficulties in using a contextual justification, and that this results in a measure of reticence on his part. Ultimately, this means that Raz does not offer a coherent account of autonomy as the central liberal concern.

The two other aspects of Raz's theory, pluralism and perfectionism, should also be examined briefly. Raz's pluralism is defined by incommensurability between our comprehensive goals. Raz's account of incommensurability rests on the idea that sometimes $\mathrm{A}$ is neither less nor more valuable than $\mathrm{B}$, and, yet, they are not the same. Regan notes that this does not prove the existence of incommensurability, merely its logical possibility. ${ }^{90}$ He makes two alternative suggestions that are not denied by Raz's account, and, yet, undermine his conclusions about incommensurability. The first concerns the fact that Raz's examples are taken from an individual point of view, rather than from the perspective of justice. A personal choice, such as whether to take a walk or read a book, may be incommensurable from the perspective of the individual involved, but this does not mean that it is incommensurable for the purposes of social value. ${ }^{91}$ It may be that their social value is subjective to the individual, but objective from the perspective of justice, and, in fact, this is quite plausible. In this way, Raz appears to fail to make the link between personal and moral incommensurability.

\footnotetext{
${ }^{90}$ Regan, op cit, above note 83, p1056.

${ }^{91}$ Ibid, p1057.
} 
Secondly, what Raz sees as incommensurable, may simply be a hard decision. The fact that people do choose between options might indicate that they are not incommensurable in the way Raz supposes. The fact that people agonize over such decisions, Regan suggests, may, in fact, show that they are commensurable, otherwise such lengthy deliberation would be pointless. ${ }^{92}$ Both of these points are valid, and show that Raz's account of incommensurability is not sufficiently explained to justify its role in explaining why autonomy is valuable in a perfectionist system.

Finally, Patrick Neal discusses the general distinction between perfectionism and antiperfectionism. He characterises this distinction as being between a liberal recognition of choosing freely and choosing correctly, or, in other words, of respecting the individual's will and respecting the individual's intellect. ${ }^{93}$ Anti-perfectionism, such as is advocated by Rawls, respects the will of the individual and is tolerant and nonjudgmental. ${ }^{94}$ However, Neal doubts that such an approach could allow for the agreement and order necessary to a theory of social organisation. ${ }^{95}$ This leads him to examine Raz's approach as an answer to this problem.

Raz's perfectionism, Neal argues, must involve drawing a line between permitted and prohibited behaviour that is objective in nature. ${ }^{96}$ This is because perfectionism is fundamental to his approach, and is not contextualised in the same way as autonomy. In other words, it is part of the foundation for government authority, rather than a consequence of the society in which we find ourselves. This means the an objective standard of action is incorporated into justice:

\begin{abstract}
What is objectionable about Raz's perfectionism, in my view, is that it acts to consecrate the acts by which the lines of inclusion/exclusion are drawn in the name of a reason which is understood to be noncontingent and noncontestable, a reason which acts, ironically enough in a theory so critical of neutralist liberalism, as a disinterested sovereign power untainted by the politics which it judges. $^{97}$
\end{abstract}

In this way, Neal argues that Raz incorporates an objective standard of reason that transcends the incommensurability between comprehensive goals and the

\footnotetext{
${ }^{92}$ Ibid, p1059.

${ }^{93}$ P Neal, 'Perfectionism with a Liberal Face? Nervous Liberals and Raz's Political Theory' (1994)

20(1) Social Theory and Practice 25, pp49-50.

${ }^{94}$ Ibid, p50.

${ }^{95}$ Ibid.

${ }^{96}$ Ibid, p51.

${ }^{97}$ Ibid, p52.
} 
contextualised account of autonomy. He suggests that it is this inconsistency that is behind Raz's reluctance to actually identify any prohibited actions. ${ }^{98}$ This argument is compelling, and seems to open Raz's theory up to the bias that should be excluded from his perfectionism.

Neal concludes with respect to the will and intellect models of liberal justice, that both are clearly flawed:

To affirm the intellect model at the expense of the will model is to have become frozen into the perspective of authority and the (entirely legitimate) concern of trying to legitimate it.... To affirm the will model at the expense of the intellect model is similarly to have become frozen into the perspective of the subject and the (entirely legitimate) concern of trying to protect it from the fallout arising from the contingencies of even "justified" authority. 99

The preoccupation of the intellect model with authority stems from the idea that consent cannot be readily used to justify it, as it is, by definition,s contrary to the (wrong) beliefs and (bad) actions of many. This means that Raz faces the difficulty of justifying his standard of reasonableness, and its effect on removing certain goals. The will model can appeal to consent, since it champions the unadulterated beliefs of individuals, but the side-effect of this is the lack of agreement or a common understanding between people.

This analysis is misleading. Rawls, for example, is the archetypal anti-perfectionist in positing a theory that is neutral between people with different conceptions of the good. However, as Neal recognises, Rawls' theory is not open to all possible forms of the good. ${ }^{100}$ It does, in fact, imply general conceptions of the good of the unencumbered self, both of which are subordinated to the right. Similarly, liberal perfectionists, such as Raz, do not enforce a particular way of life on all citizens; rather, they define some space within which individuals are able do choose their own goals and act accordingly. Both approaches suffer from the problem of legitimating restrictions on goals and action.

Where the two approaches differ is in terms of structure, or in the way that liberal space is defined. This difference, however, seem insufficient to warrant thinking of

\footnotetext{
${ }^{98}$ Ibid, pp52-53.

${ }^{99}$ Ibid, p54.

${ }^{100}$ See ibid, pp51-52.
} 
the two approaches as antithetical, or of conceiving of the perfectionist/antiperfectionist distinction as being a fundamental liberal distinction. In truth, all liberal theories of justice will be, both, open to alternative conceptions of the good, and, structurally and substantively closed; and, what is important is that this dualism expresses a valid meta-ethical approach and justificatory structure.

The Morality of Freedom is a work of political and moral theory whose subject stretches beyond the perspective given of it here. Nevertheless, it does assert the moral underpinnings of a theory of liberal justice, and, as such, it fails in a number of ways. It is significant and interesting in its explicit assertion of perfectionism, its contextualised account of autonomy, and its pervasive appeal to an incommensurable plurality of goals. However, the accounts of each of these are inadequate: Raz fails to justify autonomy as the proper focus of justice; to give a detailed account of incommensurability: and, to present a plausible and justified account of perfectionist limitations on action. Raz's work does represent a significant development from Rawlsian liberal theories, but has not gone so far as to overcome the problems these face.

\subsection{Gray's post-liberalism}

The political theory of John Gray is made up of a number of relatively distinct stages. The first of these corresponds with the publication of Post-Liberalism in 1993. In this book he affirms many of the themes developed by Raz, makes some interesting pronouncements on liberal theory, and develops the idea that value-pluralism could function as the central liberal concern.

In Liberalisms, an earlier work, Gray seems to declare his goal as being to "clear away the rubble, piece by piece, of the grand liberal theories, so as to open up a perspective in the political tradition we have inherited." ${ }^{101}$ Like MacIntyre, he views liberal theory, not as the search for a timeless, universal truth, but, rather, as a "historical singularity" that acts only to "summarize or abbreviate (very imperfectly)

\footnotetext{
${ }^{101}$ Gray, op cit, above note 33, pvii.
} 
certain passages in the historical experience of specific liberal societies". ${ }^{102} \mathrm{He}$ suggests that the way forward for post-doctrinal liberalism is to admit its own shortcomings, and reformulate itself as a historically limited undertaking. ${ }^{103}$ Gary suggests that the liberal theorist should acknowledge that

\begin{abstract}
there are few universal political dilemmas and no universal solutions. He may adopt liberal positions on a variety of questions, and even defend his own liberal order as one among the legitimate forms of political order. He may ... seek to identify the postulates of liberal society, and to give a defence of liberal political morality that aims to be compelling to his readers. Whatever he does he will not engage in the vain project of constructing a liberal doctrine. Indeed, if his inquiries have a practical aim ..., it will be to protect the historical inheritance of liberal practice from the excesses of an inordinate liberal ideology. ${ }^{104}$
\end{abstract}

Gray's attitude towards this post-liberal thought is historically-aware, and that seems to eschew the role of theory. Instead, he suggests that the proper activity of liberal thinkers is to explore liberal ideas, or a liberal system, in a way that is convincing to his or her liberal readers. This is how Gray views Raz's The Morality of Freedom: because its justification is contextual, the role of the work itself is not to reveal truth, but, rather, to convince people that his is a valuable conception of liberalism, one that is worth reproducing in liberal societies. ${ }^{105}$

In Post-Liberalism, Gray elucidates his own view of liberal contextual thought. At the centre of this is an account of value-pluralism that is dependant on our social context.

\begin{abstract}
We are none of us defined by membership in a single community or form of moral life. We are 'suckled on the milk of many nurses' ..., heirs of many distinct, sometimes conflicting, intellectual moral traditions. Further, the traditions to which we are heirs are not windowless monads, self-sufficient and fully individuated entities which (like pebbles) coexist without interacting. They are rather prisms, each of which refracts the light cast by the other traditions which environ it, and which together throw into each of us a shifting pattern of colours. The complexity and contradictions of our cultural inheritance give to our identities an aspect of complexity and even of plurality which is not accidental but ... essential to them. For us ... the power to conceive of ourselves in different ways, to harbour dissonant projects and perspectives, to inform our thoughts and lives with divergent categories and concepts, is integral to our identity as reflective beings. ${ }^{106}$
\end{abstract}

There are a number of interesting points to be drawn from this powerful statement. Gray suggests that the complexity of our cultural contexts informs the plurality of our identities. This plurality is not trivial or secondary, but is essential to our selves. This

\footnotetext{
102 Ibid, p240.

${ }^{103}$ Ibid, pp262-263.

${ }^{104}$ Ibid, p264.

${ }^{105}$ Ibid, pp266, fn 35.

${ }^{106}$ J Gray, Post-Liberalism: Studies in political thought (1993), pp262-263.
} 
applies to members of the modern western societies, since it is there that "status hierarchies are permeable, roles complex and often conflicting and social monitoring of personal behaviour intermittent and weak". ${ }^{107}$ Like Raz, Gray presents a view of liberalism that is justified only in relation to western societies. Unlike Raz, Gray focuses primarily on the pluralism of people's identities and their values.

The view of the self conveyed here is not of the Rawlsian unencumbered self; nor is it the communitarian self, constituted by the relationship to his or her community. Rather, it is one that acknowledges the importance of our cultural context. Gray's modern self is culturally constituted; but rather than embodying a single set of authoritative beliefs, it comprises a number of overlapping and potentially contradictory ideas and groups, none of which "ever claims the entirety of our life or loyalty". ${ }^{108}$

As an explanation of our modern identities, Gray's account is quite compelling. This identity-pluralism is then connected with a value-pluralism through an explanation of incommensurability. Gray adopts Berlin's account of the incommensurability between ultimate and objective, yet uncombinable ends. ${ }^{109}$ Gray is careful that his valuepluralism is objective, rather than relativist or sceptical. This means

that ultimate values are knowable; that they are many, that they often conflict and are uncombinable, and that in many of such conflicts there is no overarching standard whereby their claims are rationally arbitrable. ${ }^{110}$

Unlike Raz, Gray makes it clear that his conception of pluralism goes beyond the subjective choices of individuals, and so is relevant to an account of justice. Interestingly, despite his historical focus, Gray rejects intuitionism as relevant to ethics on the grounds that it "sanctifies the deliverances of local knowledge, however restrictive or distorted they may be". ${ }^{111}$ To complete Gray's conception of the modern self, he agrees with Raz that in our pluralist lives, autonomy is constitutive of our

\footnotetext{
${ }^{107}$ Ibid, p259.

${ }^{108}$ Ibid.

${ }^{109}$ Ibid, p290.

${ }^{110}$ Ibid, p291.

${ }^{111}$ Ibid, p294.
} 
well-being. ${ }^{112}$ Consequently, at every stage of our lives we need the freedom to choose between plural ends, and to express plural aspects of our lives.

These historical and contextual conceptions of pluralism and autonomy deny "the authority of liberalism as a universal, trans-historical and cross-cultural ideal", and leave behind "the historic inheritance of civil society that has now spread to most parts of the world". ${ }^{113}$ Civil society refers to arrangements of voluntary collective action, separate to the state, including "voluntary associations, market exchanges and private institutions within and through which individuals having urgent conceptions and diverse and often competitive purposes may coexist in peace". ${ }^{114}$ These arrangements and institutions, and the liberty they engender, are essential to liberalism. $^{115}$

The final point in Gray's account of post-liberalism is the link between the necessary pluralism and autonomy of our modern lives, and liberal civil society. Gray seems to make three separate points. Firstly, he notes that a liberal society is one where "the richest diversity of forms of flourishing is most likely to be achieved." ${ }^{, 16}$ In a related point, because a liberal society respects autonomous choice, it also promotes our western sense of well-being and partial self-authorship. ${ }^{117}$ Finally, a liberal civil society protects the epistemic freedoms of people, namely the freedoms of inquiry and expression. These are significant because they allow us to explore what he calls the 'intimations of incommensurability' suggested by interactions between cultures. ${ }^{118}$ Because of the level of these interactions, Gray asserts that this justification for liberal civil society is near-universal, meaning that his liberal conception is theoretically contextual, but in today's world would be almost ubiquitous.

Gray's conception of liberalism is persuasive in a number of ways. It builds on Raz's contextual account, but offers a more convincing sociological picture that combines pluralism, autonomy and a compelling account of the relationship between individuals

\footnotetext{
112 Ibid, p307.

${ }^{113}$ Ibid, pp313-314.

114 Ibid, p159.

${ }^{115}$ Ibid, p314.

${ }^{116}$ Ibid, p324.

117 Ibid.

${ }^{118}$ Ibid.
} 
and communities. The important central difference between the two is the focus in Gray's work on value-pluralism, as opposed to autonomy. This deepens the contextual understanding of liberal civil society.

Because his sociological account of the western, modern self is more extensive, Gray avoids some of the problems from which Raz suffers. This also allows a closer examination of the links between his account and justice. Gray argues explicitly in favour of a liberal civil society on the grounds that it promotes a rich diversity of human flourishing, allows examination of cross-cultural incommensurability, and promotes our autonomous well-being. However, he fails to explain the moral value of such a rich diversity. Even if the social fact of value-pluralism were to be admitted, this does not necessarily mean that diversity is valuable. Gray offers no other conception of diversity, or the reason for its importance.

The same applies to his examination of incommensurability. That two goals cannot be compared or combined does not lead inexorably to the conclusion that examination of the differences between the two is important. In fact, it might be argued that, in the context of incommensurability, such effort is pointless. In relation to Gray's final argument, the promotion of our well-being has definite value in relation to our individual interests; but this is not, by itself, a sufficient argument on which to base the political organisation of a society, nor would Gray want his argument to be reduced to one of contextual well-being.

Deep and compelling though Gray's conception of the modern self and his understanding of the interplay between individual and community are, these are not sufficient to support the moral conclusions he draws. Admittedly, he devotes only a minimal part of his book to these positive arguments, and describes his conception of the liberal civil society only vaguely. However, the tenor of Post-Liberalism is the peeling back of liberal-doctrine from liberal thought to see what remains; and Gray's moral account of this core liberal residue seems inadequate.

This inadequacy cannot discount the argument that, into his pluralism-autonomy framework, Gray pours his own moral beliefs and biases; an ironic conclusion since this is one of the accusations he makes against Rawlsian theory. One might reply that 
the moral arguments he makes are valid since they convey western ideals: to explore difference and to embrace diversity. This goes against Gray's assertion that intuitionism is an improper part of ethics, and would lead to a number of the problems relating to the use of social understandings in justice that were discussed in relation to Walzer's work.

Gray later distances himself from this post-liberal approach. In Enlightenment's Wake: Politics and Culture at the Close of the Modern Age (1995), he adopts an account of value-pluralism that is explicitly non-liberal. He states firstly that

[v]alue-pluralism cannot entail, or ground, liberalism in any general, still less universal way. The historical fact of a diversity of conceptions of the good, or of world-views, in a particular society may be a good reason for the adoption of liberal institutions in that society; but if value-pluralism is true, the range of forms of genuine human flourishing is considerably larger than can be accommodated within liberal forms of life. As a matter of logic alone, it is safe to say that value-pluralism cannot mandate liberalism. ${ }^{119}$

Gray states that even in the context of western value-pluralism, there is no universal grounding or necessary link between this and a system of liberalism. The reason for this is simply that liberal civil society prohibits valuable forms of life and valuable ends that, because of incommensurability, we cannot say are objectively better or worse than any other. This argument is a shift from Gray's earlier work, and he makes this shift explicit.

The post-liberal view seems to me now to be mistaken. It is mistaken in arguing that strong value-pluralism is, in contemporary historical circumstances, a good reason for the universal or near-universal adoption of a Western-style civil society, in any of its varieties. In political milieux which harbour a diversity of cultural traditions and identities, such as we find in most parts of the world today, the institutional forms best suited to a modus vivendi may well not be the individualist institutions of liberal civil society but rather those of political and legal pluralism. $^{120}$

Instead of value-pluralism forming the centre of a liberal theory of justice, Gray shifts to a non-liberal political pluralism that aims only at the pragmatic accommodation implied by a modus vivendi. This system aims at permitting forms of human flourishing that "require as their matrices non-liberal social and political structures". ${ }^{121}$ Despite this, it recognises the link between individual well-being and

\footnotetext{
${ }^{119}$ Gray, op cit, above note 34, p133.

${ }^{120}$ Ibid, p136.

${ }^{121}$ Ibid, p141.
} 
cultural contexts, so that Gray notes that, under such a pluralist structure, various cultural forms would exist. ${ }^{122}$

This conception of human flourishing is so broad as to include ways of life that are fundamentally destructive to others. For this reason it is necessary for Gray to set some minimal standard for a valid way of life or culture. In his later book, Two Faces of Liberalism, Gray suggests criteria of legitimate government. This should include, in contemporary circumstances:

a rule of law and the capacity to maintain peace, effective representative institutions, and a government that is removable by its citizens without recourse to violence...[,] the capacity to assure the satisfaction of basic needs and to protect minorities from disadvantage. ${ }^{123}$

These criteria are certainly a necessary part of Gray's system, yet they mean the incorporation of a number of seemingly universal rules, as well as limitations placed on the flourishing that an individual or group can achieve, since this flourishing cannot go so far as to undermine the legitimacy of the state. William Curtis argues that this makes Gray's modus vivendi surprisingly intolerant:

\footnotetext{
Ways of life that violate minimal standards are illegitimate, but this potentially indicts the vast majority of ways of life that one finds in human experience. For example, although, ... Gray suggests that nonliberal medieval ways of life instantiate goods and are thus legitimate, it is clear that such ways of life are illegitimate because they presuppose a feudal social order that fails to meet most if not all of the minimal standards.... In fact, it is difficult to think of regimes that adequately meet these standards over time that are not liberal. ${ }^{124}$
}

In Gray's defence, he does suggest that these are contemporary expressions of universal criteria, so that a medieval society might meet the standards relevant to their historical context. Even taking this into account, Gray's criteria do seem to describe a modern, minimally-liberal state. It seems that, in the absence of close examination and justification of each of these criteria, and an explanation of their relationship to his pluralist ideas, he might, again, be incorporating his own beliefs and understanding of liberal justice into the structure of his theory.

Gray faces a more basic problem: just as his earlier liberal pluralism depends on a necessary link between value-pluralism and liberalism, a link here is needed between

\footnotetext{
${ }^{122}$ Ibid, p137.

${ }^{123}$ J Gray, Two Faces of Liberalism (2000), pp106-107.

${ }^{124}$ W M Curtis, 'Liberals and Pluralists: Charles Taylor vs. John Gray' (2007) 6 Contemporary Political Theory 86, pp95-96.
} 
value-pluralism and modus vivendi. As with liberalism, modus vivendi is one response to the fact of pluralism, but it is not the only response. As Robert Talisse has pointed out, there is nothing "contradictory about the idea of a state promoting a single way of life while openly acknowledging that other ways of life are also good". ${ }^{125}$ In fact, this corresponds to the fate of any individual who subscribes to value-pluralism; so, it could be argued that just as a person must choose, so must a state. This is not a liberal result, but it is no less justified than a modus vivendi.

Gray's justification of his conception of modus vivendi seems to depend on the diversity of human flourishing that it would permit, more so than a liberal regime. However, the position of diversity as the overriding political value needs to be justified in a way that Gray completely fails to do. It is clear also that his system would stifle this diversity in a number of ways: by setting a moral minimum, but also by the accommodation that a modus vivendi implies. In an earlier criticism, Talisse argues that Gray's system involves reciprocity among conflicting groups:

Gray's modus vivendi depends on citizens acknowledging that the ways of life opposed to their own may realise distinct human goods, and that the proponents of these other doctrines are not necessarily in error ... [H] is modus vivendi requires that the contending ways of life respect each other. ${ }^{126}$

This requirement places demands on groups that could potentially constrain their ways of flourishing, as it dictates how each group should value their culture, and their relationship to other groups. It almost requires an acceptance of value-pluralism on a group level that seems contrary to the ideal of encouraging diversity. As Curtis comments, this "substantive ethic of mutual respect ... is difficult to square with Gray’s quest for moral minimalism". ${ }^{127}$

In all, Gray's attempt to justify a modus vivendi between various cultural groups rests on an unexamined and unjustified account of the value of permitting the greatest diversity of human flourishing. It seems to imagine a society of distinct groups dictating monism to their members, but acting as pluralists in the public sphere, a picture that is at odds with Gray's earlier and richer conception of the modern person.

${ }^{125}$ R B Talisse, 'Can Value Pluralists be Comprehensive Liberals? Galston's Liberal Pluralism' (2004) 3 Contemporary Political Theory 127, p134.

${ }^{126}$ R B Talisse, ‘Two-faced liberalism - John Gray's pluralist politics and the reinstatement of enlightenment liberalism' (2000) 14(4) Critical Review 441, p453.

${ }^{127}$ Curtis, op cit, above note 124 , p98. 
This system does not allow unrestricted flourishing, but, rather, imposes various constraints, which in turn seem to reflect his own minimally-liberal leanings. Even if he were to justify his view of diversity and overcome the various constraints, the resulting system would fail to permit all forms of human flourishing, since not all forms could conceivably ever be realised at the same time anyway. Even a wholly permissive society, for example, would prevent those forms of flourishing that, in Gray's terms, require as their matrices more restrictive social and political structures. It is always necessary to allow some, and prohibit other, forms of flourishing, and it is the nature and substance of the structure that informs the relationship between the two that is the concern of liberal theory.

\section{Liberal pluralism}

This chapter concerns the communitarian criticisms of liberalism, and the initial responses to these. In particular, it focuses on the developing and expanding role that pluralism plays as part of liberal theory. The role of pluralism in contemporary liberal theory goes back to A Theory of Justice; Rawls' approach involves recognising and permitting a broad pluralism in the domain of conceptions of the good. The difference here is that, in the wake of communitarianism, the centrality and importance of pluralism has grown. In this section some features these theories will be examined.

In structural terms, the new attitude towards pluralism has replaced neutrality as the conception that defines the liberal space within which we are permitted to act: where once freedoms would come from a state remaining neutral between conceptions of the good, now this restraint comes from the recognition of the value-pluralism of these conceptions. From a justificatory standpoint, this pluralism, as expressed in the variations between individuals, is no longer a factor for liberalism to overcome, to ignore or to correct by way of redistribution; instead, it is the very reason that liberalism is valuable as a political approach, because it acknowledges the incommensurability between persons, groups and cultures. This liberalism is not designed to overcome or manage pluralism, but, rather, it identifies pluralism as both its central concern and as the source of its moral and political value. 
In terms of liberal theory as a response to moral uncertainty, this represents a fundamental shift. Whereas the earlier theorists aim for a universal justification of a liberal theory of justice, these post-communitarian thinkers tends to employ a contextual justification that results in a less structured and less settled system of social organisation. They are careful, perhaps with the exception of Walzer, to distinguish between their contextual approach and both relativism and scepticism. The approach of liberal pluralism is that the fact of pluralism, whether contingent or universal, justifies the liberal state. If this does not imply moral relativism or scepticism, it does at the least show an incomparability between the fundamental units of political justice, which denies a closely-structured approach to political theory, and brings into question the possibility of any clear and meaningful political prescription.

\subsection{Contextual justification}

The theories of Walzer and Raz incorporate conceptions of pluralism, but without these becoming the central focus: social meaning plays the central role in Walzer's work, as does the contextual but essential value of autonomy with Raz. Together with the various works of Gray, these post-communitarian liberalisms express the themes of historicism, social meaning and contextuality, as opposed to the Rawlsian focus on theory, structure and universality. These new and closely linked themes, in effect, respond to the communitarian criticisms of liberalism. The use of historicism and social meaning shift liberalism away from the unencumbered self, and tend to adopt a position where a person's beliefs and conception of the good are part of a broader historical and social experience. The contextual justifications and historical explanations of liberalism's core values represent a shift away from enlightenment approaches to moral theory.

This new approach brings with it new justificatory challenges. Whereas earlier theories attempt at a rational and universal expression of moral justice, these contextually justified approaches derive the structure and content of justice from the shape of our western cultural values. The obvious initial requirement of this is a 
socio-historical account of the relevant aspect of western society. Even if a convincing account is rendered, there is something disconcerting about a contextual justification, and not just that the result is contingent rather that universal. Strictly speaking, all moral justification is contextual: it may depend on the nature of reality or on human nature, but all moral theory begins from some real foundation. A Theory of Justice, for example, begins from a position that is characterised by circumstances of justice, and a conception of people as morally capable beings. ${ }^{128}$

It is not the fact of contextuality, then, but rather the nature of the particular context involved that makes the resulting justification disturbing. In particular, these theories tend to depend on some essential feature of western society. Such a feature may be transitory or accidental; it may be complex and impossible to define; it may itself be the subject of significant difference and dissent. What is difficult to accept is that from the utter complexity and, as has been asserted, plurality of accepted beliefs, can be drawn a single principle of political legitimacy, from which is derived a complex and ubiquitous structure of justice. This approach seems strained. The difficulty is in pulling from the nebula of thought and understanding a single conception that is rationally articulated, and built into a great moral structure that is not merely descriptive or influential, but is the best expression of justice available.

This is clearest in relation to Raz, who uses a conception of autonomy to direct his system of morality. This autonomy is drawn from western society, and is championed enthusiastically by him. The validity of this approach is suggested by the broadness of the conception itself; autonomy or personal freedom is itself a complex and difficult ideal that, in some form, plays an underlying role in almost any conceivable and complete idea of value, and has a special normative place in western morality. Despite this, it is one conception of moral value among many; it does not even incorporate the realisation of any material end, merely the opportunity to realise.

Walzer's approach is more substantively than structurally contextual. For him, shared social understandings express principles of justice (excluding the universal separation between spheres). This approach is clearly contextual, as it depends on social

${ }^{128}$ Rawls, op cit, above note 8, p110. 
understanding, but Walzer overcomes the problem of simplifying social meaning, as he is not limited to a single conception. However, his approach would need to explain the means by which justice could be actually realised out of this social meaning. While incorporating all the complexity of social belief, Walzer fails to identify how this could be distilled into a coherent account of justice.

The approach of contextual value-pluralism has an additional attraction in that it implies a general and foundational variation in social belief and understanding. However, this brings its own difficulties. At this stage it can be concluded that socially contextual approaches to justice, however they are conceived, are not necessarily wrong, but face a substantial justificatory burden.

It should also be noted that similar problems of complexity apply in some form to any liberal theory that founds justice on a single conception or idea, as were featured in the analysis of the earlier chapters. For contextual justifications, this issue is brought into sharp focus in that differences in our actual beliefs and understandings are more readily perceptible than those of morality or human nature generally, and arguments that a single conception can faithfully represent the whole are less convincing.

\subsection{Conceiving pluralism}

The project of liberal pluralism depends on an acceptable conception of pluralism. A typical approach is to identify the persistent disagreement between people on what is to be valued, and characterise this difference as being incommensurable. This means that there is no possible means of combining the values and belief systems of a population of people into a unitary system of value such as to guide a theory of justice. From this fact of pluralism a liberal system can then be derived.

But, the fact of pluralism is by no means unimpeachable, and has been described itself as a controversial moral truth. ${ }^{129}$ On this view, the acceptance of pluralism can be seen as an admission that the fundamental nature of moral or social value is plural.

${ }^{129}$ See Curtis, op cit, above note 124, p96. 
Yet pluralists, such as Gray, while asserting the very real incommensurability between values, deny that this invariably means relativism or scepticism: that personal conceptions of value relate and are relevant only to the valuer; and that we cannot know the value of any personal set of beliefs and values.

Objective pluralism ... affirms that ultimate values are knowable, that they are many, that they often conflict and are uncombinable, and that in many of such conflicts there is no overarching standard whereby their claims are rationally arbitrable: there are conflicts among the incommensurables. ${ }^{130}$

Gray's ultimate values are real and have objective moral value, it is just that they are potentially in conflict with other values in a way that is not reasonably resolvable. This pluralism does not infer a relative or subjective value, but, rather, means an incomparability between objective values. Another value-pluralist and liberal, William Galston, takes a similar position:

Pluralism is not the same as relativism. Philosophical reflection supports what ordinary experience suggests - a nonarbitrary distinction between good and bad or good and evil. For pluralism as for any serious position, the difference between (say) saving innocent lives and shedding innocent blood is part of the objective structure of the valuational universe. ${ }^{131}$

Each of these value-pluralists takes the position that there is real, objective content to moral value, and, so, endorses pluralism without adopting a radically uncertain moral view.

These views of value themselves are substantively contestable: it could be argued that our choice of ends can in fact be compared in some meaningful way; or, that an attempt to judge between our chosen ends is not relevant to justice anyway, and that moral value consists not in what we might choose or believe, but, rather, a rational view of value informed by a conception of human or moral nature. At the very least, value-pluralism is sceptical towards a singular rational conception of what is relevant to just social arrangements. Implicit in it is the rejection of a monist moral conception, in favour of a searching for justice in relation to the ends chosen by morally capable individuals.

\footnotetext{
${ }^{130}$ Gray, op cit, above note 106, p291.

${ }^{131}$ W A Galston, Liberal Pluralism: The Implications of Value Pluralism for Political Theory and Practice (2002), p30.
} 
In this way, the conception of pluralism is itself a complex moral assertion. It cannot just be a socio-historical fact, but has significant moral consequences, otherwise it would simply be the fact of disagreement. Although it draws on our experience of conflict and the seeming implausibility of one set of values or value system being shown to be true, this is a moral conclusion and one that should not be made without justification. Within the context of liberal theory, this pluralism should be clearly explored, and its moral aspect should cohere with the remainder of the theory. The work of both Gray and Galston in this respect seem, as moral theories of value, to be tentative and embryonic.

\subsection{Pluralism and liberalism}

After laying down some conception of value-pluralism, the liberal theorist's next task is to find a viable and necessary link, whether universal or contextual, between pluralism and liberalism. The relationship between the two, as moral conceptions, is a difficult one; it can be characterised as including at once both antipathy and sympathy.

This sympathy comes from the openness of liberalism. The liberty to pursue the ends one chooses, within particular liberal boundaries, coheres closely with the idea that these ends are, by nature, plural and incomparable. The unwillingness of liberalism to promote particular ways of life expresses the pluralistic idea that none could be demonstrated to be deserving of this focus. In this regard, there is clearly much that is similar between pluralism and liberalism, from Rawls' attitude towards justice and the good, to the neutrality and the equal respect of later liberal thinkers.

The antipathy between pluralism and liberalism is expressed by Gray's later work, when he notes that liberalism could not accommodate all the plural forms of genuine human flourishing. ${ }^{132}$ John Kekes explores this matter in more depth in The Morality of Pluralism (1993). He suggests that the liberal tendency to identify one particular liberal value and prioritise it over all others is contrary to pluralism.

${ }^{132}$ Above, note 119 . 
[T] he reason why that matters is that pluralists deny that there are any overriding values. In fact, it is an essential claim of pluralism that all of our values are conditional. Indeed, the unjustifiability of regarding any values as overriding is a straightforward implication of the incommensurability and incompatibility of values, which excludes a highest value, a medium for comparing values, as well as a canonical scheme for ranking values. It seems, therefore, that liberals who regard some values as overriding cannot consistently adopt pluralism as well. $^{133}$

It is the quality of overridingness that Kekes identifies as the basic inconsistency between liberalism and pluralism. He goes on to examine neutrality-based systems of liberalism, whereby a plurality of conceptions of the good are permitted within a procedural system of justice that champions liberal values. Such a system leads him to ask why this pluralism is constrained, and why it is not "extended across the whole range of values, including the procedural and substantive ones". ${ }^{134}$ His answer is that there is no valid reason for this limitation.

Pluralists object to the liberal ideal not because of the diagnosis of danger that inspires it but because it is a misdirected attempt to avoid the danger. The way to avoid the state's favouring some values over others is not to give power to the state to enforce some procedural values as overriding, for that would merely increase the danger. Rather it is to make sure that the values the state favours will be hospitable to the flourishing of the widest possible plurality of values. And the first step toward achieving that is to make sure that the state has no overriding commitment to any particular value, be it procedural or substantive. ${ }^{135}$

Kekes' argument against liberalism is against its procedural rather than its substantive content; the latter implies neutrality between conceptions of the good, whereas the former identifies particular liberal values that prevail over and inform this neutrality. In other words, Kekes' argument is not against the openness of liberal theory, the space that its liberties define, but, rather, its closedness, and, in particular, that this closedness is achieved through the overridingness of certain liberal values, be they some conception of autonomy, equality or neutrality.

There are two initial arguments that could be made against Kekes' position. The first is that he is concerned with theories of liberalism that champion particular values, and, although there are many such forms, the type of liberalism that is most amenable to a pluralist conception is one that focuses on the fact of pluralism itself. The differences between this and older forms of liberalism do not seem to be taken

\footnotetext{
${ }^{133}$ J Kekes, The Morality of Pluralism (1993), p202.

${ }^{134}$ Ibid, p205.

${ }^{135}$ Ibid, p211.
} 
seriously by Kekes in either this book, or in his later work, Against Liberalism. ${ }^{136}$ But, within this fact lies his probable response: that even in liberal theories focussed more clearly on pluralism, there is present a drive that is not pluralistic, but is essentially liberal. While these theories might tend to incorporate pluralism more, and so be closer to Kekes' own views, they are still recognisably liberal. This reply matches up with Gray's ultimate criticism of liberalism, that it must close off some forms of human flourishing, simply because of its nature as pluralism. ${ }^{137}$

The second argument against Kekes is that the values that liberalism promotes are, in fact, closely allied to pluralism, especially in the case of liberal pluralism. Kekes identifies the core liberal (and non-pluralistic) value as being autonomy. However, autonomy itself tends to promote pluralism, in that it implies freedoms that allow plural conceptions of life to flourish. Again, Kekes (and Gray) would probably respond that these are bounded by liberal ideals, and, so, would lead to a social organisation that is essentially liberal rather than being essentially plural.

These descriptions of the affinity and antipathy between pluralism and liberalism are both valid. One general idea does result from the discussion, that is, that the relationship between pluralism and liberalism, in particular, the derivation of liberalism from pluralism, is not necessary and automatic. Instead, any theory of liberal pluralism must draw clearly the justificatory process by which liberalism is inferred from the fact of pluralism. There may be a real affinity between the two, but this is not by itself sufficient grounding for liberalism, as any such theory must also contain liberal content that is not a simple expression of pluralism, and which necessarily sets boundaries on the scope of individual choice and action.

In an important sense, liberal pluralism is best thought of as one possible response to pluralism. It is the concern and the burden of any liberal pluralist theory to show that it amounts to the best, or at least to a good, response. On these terms, the fact of value-pluralism carries no necessary social scheme, and, so, can be employed by various theories of social arrangement in various ways. This must also apply, then, to Kekes' pluralism; it must be the case that, while drawing heavily on the idea of

${ }^{136}$ E.g. J Kekes, Against Liberalism (1997), pp15-22, pp150-152.

${ }^{137}$ See note 116. 
pluralism, it is not necessarily implied by it. While it is not possible to explore closely his pluralistic theory, it is interesting to consider the general tenor of his positive arguments in order to further the idea that no single theory is implied by pluralism, and to explicate the relationship between the fact of pluralism and liberal pluralism.

Kekes' system would require that the state should

\begin{abstract}
first, use its power to protect all the procedural and substantive values necessary for all good lives and, second, make it possible for its citizens to pursue, within appropriate limits, such secondary values as they may require, beyond the primary values, to make a good life for themselves. As to how the state should do it, the pluralistic view is that it should take an active role in protecting both primary procedural and primary substantive values, and it should maintain neutrality about secondary values whose worth varies with conceptions of the good life. But taking an active role does not mean that the state should regard as overriding the claim of any particular value. It means that it should do what it can to balance the claims of all the primary values. Finally, the reason why the state should do all this is that it should be guided by a conception of its function, namely, to guarantee the conditions in which its citizens could make a good life for themselves. That conception is at once the motivating force behind, and the ultimate standard for, conflict-resolution. ${ }^{138}$
\end{abstract}

This seems to mean that the common values, those required for all conceptions of the good life, will be actively protected by the state. This would probably include those rights and freedoms relevant to the exercise of a person's choice of how to live, and presumably some minimum level of resources. When in conflict, the state will engage in a balancing exercise between the relevant values. It will also protect the secondary values, those that are desired in different ways by different people, while remaining neutral between them.

This system sounds similar to how a system of liberal pluralism would operate, but there are a few significant differences. Kekes' pluralistic perspective means that the liberties and institutions that a state would recognise and employ are attuned to pluralism, for example, the requirement that the state support an educational system that "teaches students about the plurality of values". 139

In addition to the difference of perspective, Kekes' system is notable in that, when conflicts between values are resolved, no single value or set of values overrides the others. Instead, a process of balancing values takes place, the result of which will

\footnotetext{
${ }^{138}$ Kekes, op cit, above note 133, pp213-214.

${ }^{139}$ Ibid, p215.
} 
depend strongly on the context of the conflict. ${ }^{140}$ In this way, the state's promotion of particular values will not be general, but, rather, contingent on the circumstances involved. It is this contingency, Kekes argues, that sets his pluralism apart from liberalism.

This system might be criticised broadly in that it seems to promote the idea of pluralism to the exclusion, or at least to the subordination, of other moral ideas. It is clear that, despite eschewing overriding values, pluralism is its overriding focus, and Kekes could be criticised for this reason. Such an argument is not fatal, but does require that the theorist explain why pluralism is morally prior to the plural values themselves. Essentially, it requires pluralism to be conceived as indicating the reality of social or moral value, so that it transcends other significant values.

Kekes' system seems terribly monistic, in the sense that pluralism is the only focus of justification, and that other values are subordinated in this pluralist system. As is suggested, adopting pluralism as the central politico-moral fact is itself contestable; but, even we were to agree on the importance of an irreducible moral pluralism, it is not clear that Kekes' is the system we should accept.

Firstly, it is unclear that there is sufficient common ground between conceptions on which to give substance to the primary procedural and substantive values. The idea of needing this common ground seems contrary to the singularly pluralist focus, yet Kekes makes much of unity of social values, and views pluralism as a virtue that should be taught in schools. An avowed pluralist might balk at this, and, instead, suggest a social free-for-all, where each person competes in a state of nature type environment to promote and realise their own conception of the good. Alternatively, a different 'pluralist' might call for a greater degree of social order than Kekes, and argue that the destabilising influences of pluralism need to be tempered by a societal culture and belief system, and heavy restrictions on the conceptions of the good that can be chosen. Such a person might not appear, to Kekes, to be a pluralist, but, perhaps, to the more anarchistic pluralist, neither would he.

${ }^{140}$ See ibid, p216; Kekes, op cit, above note 136, p210. 
The point here is the same one that applies to liberal pluralists, that there is no necessary link between pluralism as the central moral fact and a particular system of social organisation or theory of justice. Each of these approaches are possible, and even plausible, responses to pluralism, but they need to do more than simply identify some sympathy with it. The recognition of pluralism does not imply a system of government that focuses primarily on maximizing some conception of pluralism; any such system cannot rely entirely merely on a conception of pluralism for its justification, and to indicate its substance.

The nature of this problem is that pluralism itself does not imply any particular scheme of social organisation or of justice. There is nothing in pluralism itself that indicates whether it is something to be overcome or respected, fostered or ignored. Even if there was agreement on, say, respecting this pluralism, how this would be achieved and what other moral ideas this would entail would also be relevant. So, Kekes' balancing between the values of particular situations is itself an approach that is not inherent to pluralism, as he seems to suggest, but, rather, implies a separate and particular set of moral premises. These would include that pluralism can be advanced by making judgments between values, and that such judgments should be contextual and should vary so that no single value will ever be favoured generally.

Appealing to liberal values in order to respond to pluralism should not be criticised on the grounds that it promotes some values over others, as such a step is necessary to get from pluralism to any reasonably complete theory of social justice. What is important is that this liberal content is acknowledged by the theorist not as being derived directly from pluralism, but as a particular response to pluralism, and so one that needs to be fully justified.

\subsection{From pluralism to liberalism}

The characterisation of liberal pluralism as one response to pluralism leads to an examination of the possible justificatory paths from the latter to the former. If pluralism is to be the central focus of liberal theory, then there must be some clear 
way in which it is the basis for liberalism, or, from the other direction, in which liberalism is an appropriate response to pluralism.

One example of an avowedly pluralistic explanation of liberalism is the idea that liberal freedom and autonomy promote a diversity that accords with pluralistic ideals. This is the argument that Gray unsuccessfully makes, and that has been developed by George Crowder:

[T] acknowledge the truth of value pluralism is to endorse the promotion within a given social arena of the widest possible diversity of human values, a goal maximally satisfied under liberalism. ${ }^{141}$

Crowder sees diversity as a potential intermediate step between pluralism and liberalism. He argues that the acceptance of pluralism means that all goods that contribute to human flourishing must be endorsed equally, not because they are measurably equal, but because they are 'equally ultimate', a term he borrows from Berlin. ${ }^{142}$ From this realisation

\footnotetext{
it follows that the pluralist outlook commits us to respecting the full range of human goods.... Since all these goods must be respected equally, it follows that in any particular situation it is desirable, prima facie, that as many of them as possible be promoted. Pluralism implies the desirability of value diversity. ${ }^{143}$
}

Crowder completes the link by arguing that liberalism, understood as the "minimal framework of principles necessary to permit or enable individuals and groups to pursue divergent ways of life without coming into harmful collision", is the best means of achieving this diversity. ${ }^{144}$

But, the argument from pluralism to diversity is not as strong as he suggests. That plural and morally uncombinable goods or ends are equally ultimate, in the sense that they indicate ultimate moral value, yet cannot be usefully compared, is an acceptable interpretation of value-pluralism. From this, Crowder states that the pluralist outlook requires that they be endorsed equally. Putting aside the question of conflict, central though it is to pluralist social theory, this can only mean that one good or end cannot be given more weight than any other.

\footnotetext{
${ }^{141}$ G Crowder, 'Two Value-pluralist Arguments for Liberalism' (2002) 37(3) Australian Journal of Political Science 457, p465.

142 Ibid.

143 Ibid.

${ }^{144}$ Ibid, p466.
} 
It does not follow from this, however, that as many goods as possible should be promoted in any given situation. There is no direct line from the idea that a set of goods are equally ultimate, to the conclusion that there is value in promoting the realisation of as many goods as possible. This can be demonstrated by considering two different societies: one that is diverse in the goods that their citizens enjoy, and the other society more uniform. The uniformity in the second society is not due to monistic ideals or coercive state practices, but occurs because the individual members happen to have conceptions of the good that are more closely aligned than in the first society.

Applying the 'pluralist outlook' gives us no reason to prefer to the second of these societies to the first. Both are equally pluralist in practice, and so endorse all individual choices equally. The mere fact that the difference between these choices is greater in one society, by itself offers no justification for it carrying a greater moral value. In addition, the idea that the substance of a person's beliefs will dictate their moral value, i.e. that the more distant a person's conception of the good is from everyone else's the morally better that society is, is inimical to the pluralist spirit.

However, this is not the only conceivable argument to justify liberalism through diversity. There may be much to be made of the moral value of diversity; that there is value in some conception of difference. With further development as a moral conception, diversity might well gain the depth and resonance of ideals such as equality and neutrality. But Crowder's argument seems to be mistaken in that it attempts to derive diversity entirely from pluralism, without giving it any separate moral content. Additionally, as is the case with equality and neutrality, it is doubtful that diversity could, by itself, be sufficient to support liberalism. As a practical ideal, it would face two initial challenges: that it would tend to undermine social stability; and, that it might encourage state coercion, not to unify individual action, but to promote ways of life that are different to those of others. A conception of diversity would need to be constructed that could overcome these problems and form part of a broader justificatory structure. 
Crowder's second potential link between pluralism and liberalism concerns the virtues of practical reasoning. Pluralism, he argues, implies the necessity of making political choices from among incommensurables. ${ }^{145}$ He then identifies four virtues that would benefit this process, and claims that these are best achieved in a liberal state. These are: a generous appreciation of the broad range of valuable goods; the realist acceptance that not all goods can be achieved; an attentiveness to the context of a particular decision, including the nature of the goods and the claims on the goods involved; and, finally, the flexibility to tailor the decision to the particular situation, and, in particular, to reach a balance between general rules and particular judgments. ${ }^{146}$

The last of these virtues, flexibility, is most interesting and illuminating here. It is interesting because it reflects the exact same balancing approach that Kekes characterised as being non-liberal in nature; and yet, Crowder finds that this flexibility corresponds with autonomy, the very liberal value that Kekes was most concerned to reject. Crowder makes the argument between liberalism and this pluralist flexibility by asserting that,

[t]o be autonomous contrasts with being coerced or manipulated, and also with the uncoerced and unmanipulated acceptance of what Mill calls 'the despotism of custom', the uncritical reception of prescriptive tradition as one's ethical standard. To be autonomous is to deny that value conflicts can be resolved simply by the mechanical application of traditional and other rules. Autonomy, in its opposition to unquestioning adherence to custom, is immediately on the side of flexibility against rigidity in ethics. ${ }^{147}$

While Kekes argues that autonomy should not override any other value, Crowder suggests that autonomy itself encourages a pluralist flexibility that seeks a balance between various goods. This conflict illustrates how Kekes is concerned with arguing against pre-pluralism forms of liberalism, and has not recognised how the natural affinity of the core liberal values with pluralism might be used to justify a liberal pluralism. It also recalls the burden Crowder faces to explain why the liberal ideals are justified by the pluralism he admits. Here, he attempts to show that the flexibility required by political decision-making in a pluralist context is best achieved by a liberal state, since it aligns with the autonomy such citizens enjoy.

\footnotetext{
145 Ibid, p468.

${ }^{146}$ Ibid, pp468-469.

${ }^{147}$ Ibid, p469.
} 
The distinction he uses to characterise this argument is between the uncritical application of customary or other rules, and a process of applying the principles and judgments that one considers appropriate in all the circumstances, arrived at by the exercise of autonomy. This latter approach, Crowder argues, is the reasonable response to value pluralism, and is engendered by the liberal state.

However, this is not as straightforward a result as he suggests. It should first be noted that Crowder is no longer concerned here with pluralism as a reflection of moral reality, but, rather, with pluralism as the central concern of political judgment. This shift in focus implies not only that moral value is plural and incommensurable, but that people should, both, be conscious of this, and, act in a reasonably consistent way when making political decisions. This may seem innocuous, but one of the central ideas of pluralism is that it takes people as they are, and implies no coercion of belief or judgment. Essentially, Crowder is arguing for a pluralist ethic, at least in the political sphere, to which all should subscribe. As William Galston eloquently puts it, "[t]o demand that every acceptable way of life reflect a conscious awareness of value pluralism is to affirm what value pluralism denies - the existence of a universally dominant value". 148

Perhaps that statement is too strong here, as Crowder might argue that he is in favour of political and not personal recognition of value pluralism. The fact remains, however, that he imports as part of his conception of the person a conscious appreciation of pluralism, and reasonableness of choice and action in response. This does not mean that the resulting liberalism has no valid link to value pluralism, but it does represent a new aspect of liberal pluralism that would require the conception of the pluralist state be developed considerably further, in order to incorporate a pluralist consciousness and ethic. Arguments concerning a possible split between the political and the personal find a fuller expression in Rawls' political liberalism, which will be discussed in the next chapter.

\subsection{Galston's Liberal Pluralism}

\footnotetext{
${ }^{148}$ Galston, op cit, above note 131, p53.
} 
Galston, himself, in Liberal Pluralism, posits a theory of liberalism that is founded on a universal conception of value pluralism as a description of moral reality. The connection between the two seems to consist of one primary and two ancillary links. The ancillary links will be discussed in dues course. The primary connection concerns the nature of rational choice in the pluralist situation.

\footnotetext{
Value pluralism suggests that there is a range of indeterminacy within which various choices are rationally defensible.... Because there is no single uniquely rational ordering or combination of such values, no one can provide a generally valid reason, binding on all individuals, for a particular ranking or combination. There is, therefore, no rational basis for restrictive policies whose justification includes the assertion that there is a unique ordering of value. If value pluralism is correct, then as Steven Lukes puts it, "For the state to impose any single solution on some of its citizens is thus (not only from their standpoint) unreasonable."149
}

Galston's perspective is from the justification of coercive state action. If incommensurable values and goods cannot be ordered in a rationally justifiable way, then the state cannot promote such an ordering; to do so would be to act in a coercive way without any rational justification. This acts as a presumption: that a state must justify its actions with good reasons. In the absence of such reasons, pluralism offers a strong argument for negative freedom, in that the state should not interfere in the choices and lives of citizens. ${ }^{150}$

Galston does recognise that the state will have the power to decide matters of pure coordination, where it matters not what the rule is but only that there is an accepted rule (such as the side of the road on which to drive). ${ }^{151} \mathrm{He}$ also recognises the inevitability of conflict, yet argues that this does not demonstrate the bias of the system or the government, but, rather, that it is the "structure of particular contexts of decision" that necessarily limit the potential choices and possibilities that individuals can enjoy. ${ }^{152}$

Talisse persuasively argues that Galston's liberalism does not follow value pluralism. In doing so, he considers three possible ways of life, which he labels A, B and C. A represents a Millian life of civic engagement, B a life of quietude and orthodoxy, and

\footnotetext{
${ }^{149}$ Ibid, pp57-58.

${ }^{150}$ Ibid, p58.

${ }^{151}$ Ibid.

${ }^{152}$ Ibid, p59.
} 
$\mathrm{C}$ an Emersonian life of self-sufficiency. He argues that pluralism does not deny the state the power to promote one of these ways of life.

The state indeed has good reason to promote, for example, A, namely that $A$ is good. Of course, the value pluralist will insist that the state has no better reason for promoting Millian civic liberty rather than religious devotion or Emersonian self-sufficiency, but surely this is not a reason for remaining neutral with regard to these options, and it is not a reason to not promote Millian civic liberty. After all, ex hypothesi, the Millian way of life is actually good. What could be a better reason for imposing it? ${ }^{153}$

Talisse argues that pluralism does not entail negative liberty in the way Galston suggests. Although there is no better reason for promoting one way of life rather thananother, that does not necessarily imply a neutrality between the two, when in fact there is value in promoting either one.

Galston responds to this argument by emphasising that he is concerned with a presumption against interference that a state must, by power of its reasons, overcome. The value of this presumption stems from the fact that a particular way of life is actually chosen by an individual.

So it is not good enough to say that in the abstract, there is nothing to choose between $\mathrm{T}$ and its alternatives, because the fact that some members of society endorse the alternatives means that there is something to choose between them. The rulers must then offer a reason sufficiently compelling to overcome the weight of endorsement. ${ }^{154}$

Galston uses value pluralism in this morally minimal way to indicate a presumption in favour of any way of life that is valued within a society, which means that no other way of life will be favoured over it in the absence of good reasons to do so. The nature of incommensurability dictates that such reasons will not come simply because of the preference of a particular individual or group for one way of life over another.

Galston's link between pluralism and liberalism appears to be robust. The nature of moral value, that it is plural and incommensurable, offers weight to any conception of life held by an individual or group in society. This weight prescribes non-interference, unless reasons of greater weight justify otherwise. This approach has a number of virtues: it connects liberalism with the essence of pluralism, the moral value attaching to various individual beliefs and choices; it is pared back, in that it links only to a bare

\footnotetext{
${ }^{153}$ Talisse, op cit, above note $125, \mathrm{p} 134$.

${ }^{154}$ W A Galston, 'Liberal Pluralism: A reply to Talisse' (2003) 3 Contemporary Political Theory 140, p146.
} 
conception of negative liberty, and, so, infers no more liberal content; and, it represents the simplest of actual connections, between moral value and noninterference.

The connection is so simple that it does not even need Talisse's three person society in order to show its central argument. Because it is logically prior to Galston's account of conflict between people, a one person society is quite sufficient. That person holds a personal conception of human flourishing, and desires to follow that conception in his or her own life. The reality of value-pluralism, Galston is in effect arguing, means that his or her conception has a positive moral value, and the opportunity for the citizen to follow that conception is similarly valued. In this way, moral value must also attach to the non-interference of his or her actions.

Once this conclusion is reached, it is easy to see how non-interference, or negative liberty, becomes a presumption that one must have good reasons to rebut: it applies to one person in the same way that it does to a vast population. The immediacy of the link between value pluralism and liberty is clear, but this reasoning does involve some assumptions in addition to a simple account of pluralism. It assumes, for example, not simply that morality is by nature plural, but that it is a form of pluralism that attaches to each person and their beliefs. It is unquestioning in the sense that it treats any actual beliefs as positive value. This is not a necessary consequence of pluralism: there is a difference between the shape of moral value being plural and incommensurable, and the judgment that this shape traces around each individual, so that each person's beliefs have some moral value uncombinable with others.

The point here is not that someone may have harmful beliefs, as these will be excluded once rules on value conflicts are enacted. Rather, what is in question is the unthinking acceptance that is entailed in this approach. Consider, for example, that a state does place substantial constraints on the one citizen, which leads him or her to adopt new beliefs on flourishing. This would be a morally bad move presumably; yet, the new beliefs cannot be said to be any better or worse than the old, as the two are incommensurable. Galston might argue that unconstrained beliefs are more valuable, which would be a morally plausible move, however this argument incorporates the 
liberal ideal of non-interference directly into the conception of pluralism, and, so, presupposes the bonds between the two that he is trying to forge.

His account is also muddied by the formulation of non-interference as a presumption. Although this keeps the connection pared back, it means that other reasons can overrule the principle against interference. This moral weighing-up seems contrary to Galston's description of moral value as being incommensurable. He does not discuss potential reasons in any detail, but it is enough to recognise that they could arise from the values and choices of other individuals, or from some separate moral concern. ${ }^{155}$ If the latter, then there is no reason to use Galston's presumption as the starting point or focus, as other factors can be equally relevant. If the former, then we are left with Talisse's argument that a state might choose one form of human flourishing despite being aware that others also have value. ${ }^{156}$

In addition, conflicts of value will often require that a choice be made. Galston's link from pluralism to liberalism may not make sense in these circumstances. The circumstances of society necessitate restrictions on the exercise of a person's conception of life, a fact that is central to any account of justice. Rawls, for example, includes scarcity as one of the underlying circumstances of justice, and this conflict is implicit to theories of distributive equality. This suggests that Galston may not be able to make a viable link from pluralism to liberalism without some account of how value conflicts might be resolved. However, as was evident in relation to Kekes' approach, there is nothing inherent in pluralism to direct the conflict-resolution process.

Although Galston's primary link between pluralism and liberalism follows the natural progression from identifying moral value to not interfering with it, it is too simple to succeed on its own. This shortcoming could only be corrected by incorporating other (liberal) ideals into his conception of pluralism or the connection he perceives between the two. Although this may result in a viable liberal theory, it is not one that is founded wholly on pluralism.

\footnotetext{
${ }^{155}$ C.f. ibid, pp30-35.

${ }^{156}$ Ibid, p35.
} 
Galston makes two ancillary arguments to link pluralism to liberalism. The first concerns the importance of diversity, although his claim is quite different to that of Crowder or Gray. He argues that a diversity of forms of human flourishing characterises our natural and pre-cultural selves. Cultural self-determination operates to circumscribe the opportunities of people to live in a manner consistent with their flourishing. ${ }^{157}$ A liberal society represents the best response to this in that it allows, to the maximum extent possible, various forms of human flourishing. ${ }^{158}$

This argument begins with a conception of our pre-cultural selves as having boundless, or at least very diverse, conceptions of human flourishing. Although culture and society set boundaries on the forms that our lives can take, this does not necessarily represent a decrease in our modes of flourishing in the manner he suggests. Instead, it is reasonable to accept that a pre-cultural situation itself involves conflicts and limitations on our life conceptions, and it is plausible to conceive of society as giving meaning to, as well as limiting, our flourishing. In this way, the shift from pre-cultural to cultural life has a complex effect on diversity. Galston fails to discuss adequately this effect, and to link this discussion to the relationship between diversity and moral value.

It is interesting to compare the conceptions of diversity of Galston and Crower. The former is concerned not with the actual realisation of different ideas of human flourishing, but, rather, with the opportunity or the space in which people can hold conceptions of flourishing. As such, it is not an argument from diversity at all, but, rather, one that relates value pluralism to the freedom of choice implicit in liberal societies. Again, the mere comparison with our pre-cultural selves seems to have no necessary moral consequence; in this way, comparison with our pre-cultural selves seems false and meaningless, and an inadequate ground for the justification of liberalism.

Galston's second ancillary argument consists of the practical point that once a society becomes liberal, and its citizens enjoy liberal forms of flourishing, it is extremely

${ }^{157}$ Galston, op cit, above note 131, p60.

158 Ibid. 
difficult to return that society to a pre-liberal, and less diverse structure. ${ }^{159}$ To do so would involve a very costly repression and dissent. This is not particularly strong as a positive grounding for liberalism; it relies on a liberal conservativism, and the high resource costs of such a change (presumably Galston would support liberalism as strongly even if technology had advanced to the point where dissent could be crushed cheaply). But, it is interesting in that it views pluralism, and implicitly liberalism, as a kind of endpoint in social development.

It is tempting for a liberal pluralist, especially one who is contextual and historical in approach, to see pluralism as a social endpoint: the result of the contracting of the world through increased technology and information exchange; and the comprehensive and complex interaction between various peoples, as defined by trade and commerce, travel and immigration, and various other forms of cultural contact. This is not to say that it is impossible for different social forms to resist or reject a pluralist ideal, that a pluralist state cannot change to another form, or that there is nothing beyond pluralism. It does, however, endue pluralism with the force of a certain inevitability: being confronted with difference in a very real way, we will tend to accept the validity and power of that difference. This point is interesting here because it does give contextual justifications of liberal pluralism some of the power of inevitability, and, so, their own form of universality, not across all possible societies and cultures, but as a consequence of the circumstances of today's world.

\section{Conclusion}

Communitarian criticisms posed a number of important challenges to liberalism; they identify the liberal assumptions inherent in the underpinnings of the early contemporary liberal conceptions, and exposed acy of aiming for a rational and complete universal moral theory. The main thrust of the liberal response canvassed here involves a change in approach: the shift from universal to contextual and historical justificatory structures; an appreciation of the importance of social forms and meanings; the discarding of anti-perfectionism and neutrality claims; and, a shift

${ }^{159}$ Ibid, p61. 
away from an unquestioning faith in theory and complex theoretical structures. This tends to result in political principles that promote ideals of toleration and basic liberal values, rather than closely reasoned conceptions of equality or boundaries on legitimate action.

One of the chief features of this post-communitarian liberalism is centrality of pluralism as a liberal concern. Although pluralism is itself complex, its most common liberal conception is as a fact about moral value: that it is, in nature, plural or multiple, and that this plurality cannot be effectively combined or measured in terms of a single metric. This means that people can hold various understandings of human flourishing or of the ultimate ends of life, or can desire various goods, and, that these cannot be reduced to a single conception or measure of the human good to which a state could commit.

Pluralism of this form is at least a partial denial of some of the underlying ideas of communitarianism: that the self is constituted by its social ties, and these ties are common between the members of a society. The potential diversity of pluralism allows self-creation and denies the promotion of group over individual belief. This form of pluralism is an essential condition of the theories of both Walzer and Raz, although the former uses the pluralism of goods in a communitarian way, subject to the shared understandings of the community's members, and the latter takes pluralism as the subject of a contextual account of autonomy.

In Gray's earlier work, and in the work of thinkers such as Crowder and Galston, liberalism is actually drawn from and founded in pluralism. The adoption of pluralism as the central justificatory focus of liberal theory marks a stark shift in approach. Instead of a universal rational moral theory that adjusts and compensates for difference, this entails deriving liberalism from irreducible differences in belief and understanding.

This change in justificatory approach implies changes in the justificatory burdens. The basic challenge of a theory of justice founded on pluralism is to make something singular or universal out of an uncombinable plurality. This is especially difficult if pluralism is held to be the ultimate moral truth or shape of moral value. The 
advantage of attempting to derive liberalism from pluralism is that there is a clear affinity between the two, corresponding to the openness of liberalism: liberal societies allow their citizens considerable freedom to pursue their own conceptions of the good or of human flourishing.

However, as this chapter demonstrates, this affinity is not enough on its own to justify liberalism. Liberalism as a system is also closed; in a social sense in that it prohibits some behaviour, and limits the opportunities of some to pursue their conceptions of human flourishing; and, in a philosophical sense in that the liberal space is itself defined by a set of principles and justifications that deny alternative ideas and conceptions. Liberalism must do more than identify the common motifs between itself and pluralism. It must also do more than identify some value or virtue that pluralism supposedly prescribes, and that liberalism delivers. This is not a problem of liberalism alone; political pluralism is not justified directly and completely by value-pluralism. Value-pluralism does not itself imply a system of political organisation. Liberal pluralism needs to acknowledge this, and to envisage itself as one response to pluralism. This means that it is not simply derived from value-pluralism, and must draw moral power from elsewhere.

The problem with requiring this additional moral content is that this seems to undermine the idea of pluralism itself, since it would mean prioritising the value of that content over others. Talisse makes a similar point in relation to Galston's theory:

[I]n order to produce a comprehensive theory of liberalism, Galston must commit to some fundamental value that provides the foundation for the legitimacy of the liberal state. However, the identification of such a value is inconsistent with value pluralism. ${ }^{160}$

He concludes that the acceptance of value pluralism is incompatible with a "robust philosophical foundation" for liberalism. ${ }^{161}$ So, the fundamental problem with political expressions of pluralism is that pluralism by itself implies no political system, and adding any substantive moral content is contrary to pluralism itself. Talisse suggests that one of the possible responses to this is to reject liberalism in favour of a pluralist approach, as does Gray. ${ }^{162}$ However, as the earlier examination of

\footnotetext{
${ }^{160}$ Talisse, op cit, above note $125, \mathrm{p} 136$.

161 Ibid.

162 Ibid.
} 
Kekes' work shows, this approach faces the same difficulty; and, in fact, any substantive political theory could not help but transgress the incommensurability of the plurality of values.

Liberalism could respond to this difficulty in two ways. The first is to re-conceive and downplay the significance of pluralism. The inconsistency between value-pluralism and a positive political theory depends upon the conception of pluralism as explicating the true form or shape of morality. By reframing pluralism so that it is not so morally foundational, or making it one part of a larger theory of morality, this inconsistency would be avoidable. The plurality of moral value could become, for example, a plurality of doctrines whereby the complexity and context of each doctrine or tradition means that different doctrines could not be combined. This is a fact about the shape of the value of doctrines, rather than morality generally, and does not prevent one being preferred over another for separate moral reasons, or in order to promote different values. In this way, the scope of pluralism might be limited to a particular sphere that amounts to something less than the whole of morality.

The second approach is to superimpose values or structures on the simple pluralismliberalism approach in the face of the inconsistency this necessarily implies. In order to justify whatever political approach is asserted, this inconsistency must be explained. One possible explanation is that of necessity: if pluralism is to be maintained as true, then the inconsistency must be set aside in order for a theory of social organisation to be suggested or supported. This pragmatic argument is powerful but disconcerting: powerful in that the practical need to organise complex social structures in a moral and effective way is strong, but disconcerting in that the truth of moral value is simply ignored in order for this to be achieved.

A possible approach to reconciling these ideas may be revealed by a further examination of the nature of choosing between incommensurables. Some suggest that incommensurability is synonymous with being uncombinable, that there is no common scale by which values can be measured, but that values can in some way be usefully compared. Pluralist theories all seem to allow that choice between incommensurables is possible, and, in fact, is the situation that autonomous 
individuals face in deciding what set of beliefs and what conception of human flourishing to endorse.

Talisse is interesting here in that, even though he holds that liberal pluralism can have no valid foundation, he states that there is "nothing contradictory about the idea of a state promoting a single way of life while openly acknowledging that other ways of life are also good". ${ }^{163}$ This seems to suggest that states can make choices in much the same way as individuals do, and that such choice is not contrary to morality.

If this is the case then it seems to leave pluralism and incommensurability with little practical meaning. A more coherent reading of value pluralism is that, although states can make a choice between incommensurable conceptions, such a choice would be based on parochial rather than rational grounds. As such, there would be no generally acceptable or universal reason for choosing one conception over another. Or, perhaps such reasons could exist, but would need to come from outside the scope of incommensurability of values. A value that is common between individuals, for example, or that is present within part of a particular social context might well qualify as a valid reason for that value. However, as mentioned above, one of the problems of contextual justifications is that the relevant value is seldom unanimous, and, in a pluralistic world, there seems to be no way to attach value to the weight of public opinion; there seems no rational reason for a state to prefer the common view of a million against the belief of one.

The only way to accept that there can be rational reasons for particular social arrangements in a pluralistic world is to view the plural nature of morality as being a foundation that can support additional structures that say something relevant about moral value. These cannot be derived directly from pluralism, but must cohere with its tenets. The nature of this relationship is a difficult one, as it is difficult to conceive how pluralism can have substance and yet admit of other substantive ideas. Arguably, this could not be achieved without some reformulation of the pluralist conception.

${ }^{163}$ Ibid, p134. 
Value pluralism offers an interesting view of moral uncertainty. It does not necessarily imply uncertainty in the epistemological sense, or scepticism, but it does marry up with one of the themes of uncertainly, as an expression of moral contestability. The idea of pluralism as the central moral fact is compelling in that it corresponds with the experience of seemingly irreducible difference of moral opinion and value. In this form it is a fundamental fact about rational morality and is consistent with the liberal freedom to choose and to be autonomous beings.

However, pluralism in this form is much more troublesome than it first appears; the contestability of values does not limit itself to individual beliefs, but extends to liberal values such as autonomy and equality and, superficially at least, threatens any political response. Liberal pluralists have not recognised sufficiently this fundamental difficulty, and, so, have taken few steps towards building a justificatory structure that defines an appropriate and mutual relationship between liberalism and pluralism. Pluralism has been conceived too easily and applied too readily, in particular, without an adequate account of the meaning of incommensurability and choice. Ultimately, it is difficult to draw from an irreducible moral pluralism a singular, unified political conception of social organisation and justice, and the attempts of liberal theorists to use pluralism in this way have been not just failures, but failures that give no indication of possible avenues for development. 


\section{POLITICAL LIBERALISM AND PUBLIC REASON}

\section{The political and the metaphysical}

Rawls' later work responded to communitarian criticisms. From around 1980 his conceptions of justice shifted focus, culminating in the publication of Political Liberalism in 1993. Perhaps the best-known of the essays he published in the intervening period, and certainly the one that best reflects the tenor of this shift, is 'Justice as Fairness: Political not Metaphysical' (1985). ${ }^{1}$ Here, Rawls is concerned with explaining why his conception of justice is not dependant on potentially controversial philosophical claims, including "claims to universal truth, or claims about the essential nature and identity of persons."2 This assertion would answer or, rather, undermine the two communitarian criticisms presented in the previous chapter, as to an individualistic conception of the self, and the enlightenment aim of finding a universal moral theory.

Rawls' basis for these claims is that justice as fairness is meant as a political rather than a philosophical or metaphysical conception of justice. ${ }^{3}$ This means, firstly, that it is contextual; justice as fairness is constructed by Rawls to apply to what he calls modern constitutional democracies. ${ }^{4}$ The result is still an expression of justice, yet is valid only in that particular societal context.

[A]s a practical political matter no general moral conception can provide a publicly recognized basis for a conception of justice in a modern democratic state. The social and historical conditions of such a state have their origins in the Wars of Religion following the Reformation and the subsequent development of the principle of toleration, and in the growth of constitutional government and the institutions of large industrial market economies. These conditions profoundly affect the requirements of a workable conception of political justice; such a conception must allow for a diversity of doctrines, and the plurality of conflicting, and indeed incommensurable, conceptions of the good affirmed by members of existing democratic societies. ${ }^{5}$

\footnotetext{
${ }^{1}$ In S Freeman(ed.), John Rawls: Collected Papers (1999), first published in Philosophy and Public Affairs 14 (1985) 223.

${ }^{2}$ Ibid, p388.

3 Ibid, p389.

${ }^{4}$ Ibid.

${ }^{5}$ Ibid, p390.
} 
Rawls asserts that no general moral conception will be acceptable as a conception of justice in a modern democracy. His target here is not acceptability; he is not arguing that we will never be able to agree on a conception of justice, but rather that such a conception is not going to reflect a single moral account. This is because moral accounts are plural and incommensurable, and our democratic history is not one of championing one of these accounts at the expense of others, but of accepting this plurality and exercising political toleration. In this way, Rawls is not disavowing the possibility of an agreed conception of justice, but, rather, emphasising that such a conception must be pragmatic and general, and must acknowledge and incorporate, rather than overcome, the irreducible pluralism inherent in democratic societies; in other words, such a conception must be political and not metaphysical. This conception, Rawls emphasises, is founded on the "informed and willing political agreement between citizens viewed as free and equal persons", rather than on a philosophical justification asserting some universal truth. ${ }^{6}$ As a contextualised conception of justice, it incorporates a similarly contextualised conception of the person, based on the idea of the individual as a "fully cooperating member of society", and a free democratic citizen. ${ }^{7}$

It is difficult to conceive of these ideas simply as clarifying what was already present in A Theory of Justice: the essay, 'Justice and Fairness', appeared some fourteen years after the book's publication, and as the result of changes in emphasis and view. ${ }^{8}$ Even though this approach is more congruent with the intuitionism implicit in the reflective equilibrium (and, potentially even, the original position), it is difficult to reconcile this political approach with the perspective of eternity, from which we are able to "regard the human situation not only from all social but also from all temporal points of view". ${ }^{9}$ Nor does Rawls present in A Theory of Justice the historical or sociological account that would be expected from a contextual justification. It seems, then, that Rawls' approach has altered substantially between A Theory and Political Liberalism; perhaps the seeds of the later approach were sewn in the earlier work, but the shift to the political was neither obvious nor trivial. This new approach represents a

\footnotetext{
${ }^{6}$ Ibid, p394.

${ }^{7}$ Ibid, p397.

${ }^{8}$ Ibid, p388.

${ }^{9}$ J Rawls, A Theory of Justice (revised ed., 1999), p514.
} 
fundamental shift in liberal theory that helped to initiate the pluralism discussed in the previous chapter. With these value-pluralist positions, Rawls shares a focus on the fact of pluralism as the main engine of liberalism, a historical and contextual approach to justification, a strong emphasis on the importance of toleration, and downplaying of the role of theory.

Political Liberalism requires a separate examination. That the principal contemporary theorist of liberalism has eschewed truth as a valid aim for political theory is itself startling. More than this, Rawls presents a complex theoretical system that incorporates a number of important and interesting parts, and that has influenced much of the most recent liberal thought.

\section{Political Liberalism}

\subsection{The political and the reasonable}

Rawls presents Political Liberalism as the response to a substantial problem in $A$ Theory of Justice. This problem is one of the stability of the resulting society. ${ }^{10}$ The problem is that a modern democratic society is characterised "not simply by a pluralism of comprehensive religious, philosophical, and moral doctrines but by a pluralism of incompatible yet reasonable comprehensive doctrines." ${ }^{\prime 1}$ This pluralism would, Rawls argues, undermine the potential stability of a society governed by the two principles of justice indicated by justice as fairness. The reason for this seems to be that justice as fairness is itself a comprehensive doctrine, and, so, is potentially incompatible with the reasonable beliefs that other democratic citizens adopt. This incompatibility would undermine the general agreement with justice as fairness that $A$ Theory of Justice requires.

Liberalism, then, must set aside the search for a comprehensive doctrine that all would accept, since any such conception could always be reasonably rejected. Instead,

\footnotetext{
${ }^{10}$ J Rawls, Political Liberalism (1996), ppxvii-xviii.

${ }^{11}$ Ibid, pxviii.
} 
it should adopt a political approach to liberalism that does not reflect a particular philosophical position, but, rather, "leaves these to be answered in their own way by different comprehensive views". ${ }^{12}$ Liberalism should focus, instead, on how to work out

a political conception of political justice for a (liberal) constitutional democratic regime that a plurality of reasonable doctrines, both religious and nonreligious, liberal and nonliberal, may endorse for the right reasons. ${ }^{13}$

Rawls' political system works within a democratic state, but is supposed to apply not just to those with a liberal predisposition. The important requirement here is not that the citizens are liberal, but that they are reasonable, and that, in agreeing to a political conception of justice, they are acting for the right reasons, a point that will be discussed in relation to public reason.

The socio-historical foundation of a system of political liberalism, then, is a reasonable pluralism of comprehensive doctrines. The idea of reasonableness is important not only in this sense, but is weaved throughout the theory. Rawls describes reasonable pluralism as being

the fact that among the views that [free institutions] develop are a diversity of reasonable comprehensive doctrines. These are the doctrines that reasonable citizens affirm and that political liberalism must address. ${ }^{14}$

Rawls later describes reasonable comprehensive doctrines as corresponding to the exercise of theoretical reason that "covers the major religious, philosophical, and moral aspects of human life in a more or less consistent and coherent manner"; they organise our values so as to reflect a cogent world view; and they will normally draw upon "a tradition of thought and doctrine". ${ }^{15}$ In terms of giving content to the idea of a reasonable pluralism, it is more useful to look at Rawls' definition of a reasonable person.

\footnotetext{
${ }^{12}$ Ibid, pxxix.

${ }^{13}$ Ibid, pxli.

${ }^{14}$ Ibid, p36.

15 Ibid, p59.
} 
There is some debate over exactly what constitutes Rawls' reasonable person, but there are at least three elements. ${ }^{16}$ The first of these covers the normal moral capacities, as were relevant to $A$ Theory, namely the capacity for a sense of justice on one hand, and a conception of the good on the other. ${ }^{17}$ The second refers to the person's willingness to cooperate:

Persons are reasonable... when... they are ready to propose principles and standards as fair terms of cooperation and to abide by them willingly, given the assurance that others will likewise do so. ${ }^{18}$

It is a feature of the reasonable person that, although they might subscribe to a particular comprehensive doctrine, they are willing to cooperate on fair terms with others who do not share the same comprehensive views. This is essential to Rawls' political liberal conception. Finally, a reasonable person accepts what Rawls calls the burdens of judgment, and their relevance to the exercise of political power. These help explain why plural comprehensive doctrines are incompatible, and the consequences of this for pluralist political life. In the words of James Boettcher, the burdens of judgment

imply that sincere and generally reliable reasoners sharing a common human reason will nevertheless often fail to reach agreement about many religious, philosophical and even political issues. Our practical reason is burdened, as it were, with a number of imperfections. ${ }^{19}$

Rawls includes in these imperfections such difficulties as: assessing complex evidence; assigning weight to valid considerations; applying vague concepts; and the effect of having different backgrounds on judgments. ${ }^{20}$ These burdens of judgment make agreement between comprehensive doctrines impossible, and acceptance of them is part of the makeup of the reasonable democratic citizen.

It is the reasonable person, aware of the complexities and limitations of judgment and willing to enter into fair cooperation with other reasonable people, who characterises Rawls' reasonable pluralism. The central assertion of Political Liberalism is that reasonable pluralism allows a political conception of justice that is not derived from a particular comprehensive doctrine or group of doctrines.

\footnotetext{
${ }^{16}$ See L Wenar, 'Political Liberalism: An Internal Critique' (1995) 106 Ethics 32, p37; c.f. J W Boettcher, 'What is Reasonableness?' (2004) 30(5-6) Philosophy and Social Criticism 597, pp604-606.

${ }^{17}$ Rawls, op cit, above note 10, p81.

${ }^{18}$ Ibid, p49.

${ }^{19}$ Boettcher, op cit, above note 16, p605.

${ }^{20}$ Rawls, op cit, above note 10, pp56-57.
} 
For rather than confronting religious and nonliberal doctrines with comprehensive liberal philosophical doctrine, the thought is to formulate a liberal political conception that those nonliberal doctrines might be able to endorse. ${ }^{21}$

It is this endorsement or acceptance of the political conception of justice by the reasonable citizens of a modern democratic society that justifies its operation. This political conception is given practical expression by the two mutually reinforcing ideas of overlapping consensus and public reason, both important parts of Rawls' theory.

An overlapping consensus represents what is common in a political conception of justice between reasonable comprehensive doctrines. ${ }^{22}$ In other words, it is the political core that all reasonable people can endorse, regardless of their actual comprehensive beliefs. It is this consensus that informs and shapes a political conception of justice, and through which reasonable pluralism can find unity and stability. This unity is not concerned with comprehensive beliefs, but rather with constitutional fundamentals.

Since we seek an agreed basis of public justification in matters of justice, and since no political agreement on those disputed questions can reasonably be expected, we turn instead to the fundamental ideas we seem to share through the public political culture. From these ideas we try to work out a political conception of justice congruent with our considered convictions on due reflection. ${ }^{23}$

This agreement is not, Rawls argues, a modus vivendi, a mere pragmatic accommodation. Rather, it is a moral conception that is drawn from the democratic culture of a society and the comprehensive beliefs of each person. ${ }^{24} \mathrm{~A}$ political conception of justice as revealed through an overlapping consensus is not, then, a compromise that an individual accepts, but, rather, it relates to each person's comprehensive view, albeit in potentially different ways. ${ }^{25}$

In order to achieve this political conception of justice, Rawls employs a conception of public reason.

\footnotetext{
${ }^{21}$ Ibid, pxlvii.

${ }^{22}$ Ibid, pp144-145.

${ }^{23}$ Ibid, pp150-151.

${ }^{24}$ Ibid, p147.

${ }^{25}$ Ibid, pp170-171.
} 
[I]n a democratic society public reason is the reason of equal citizens who, as a collective body, exercise final political and coercive power over one another in enacting laws and in amending the constitution. ${ }^{26}$

This approach extends to fundamental decisions, such as on constitutional essentials and matters of basic justice. ${ }^{27}$ The standard that such decisions must reach is defined by a liberal principle of legitimacy, which requires they be made according to rules that people may be reasonably expected to endorse "in the light of principles and ideals acceptable to them as reasonable and rational". ${ }^{28}$

In this way, public reason seems to have two roles: firstly to set the rules by which governmental power can be legitimately exercised; and, secondly, to work according to those rules to determine matters of basic justice. These allow the overlapping consensus and democratic culture of a society to manifest themselves in a political conception of justice. The subject of such a conception, Rawls asserts, would primarily include a set of basic liberties, an explanation of the priority of these, particularly in relation to matters of the good, and such measures that are required to ensure these liberties can be effectively enjoyed. ${ }^{29}$

Rawls notes that the substance of these elements, although liberal, is, conceivably, quite varied. ${ }^{30}$ It is the nature of political liberalism that it does not specify a particular principled system of justice. If it did, it would not be political, but, rather, a comprehensive liberalism, and would suffer from the same problems as does justice as fairness: it would be the subject of reasonable disagreement, and this would undermine unity and stability. Nevertheless, Rawls believes that justice as fairness, as expressed by the two principles of justice, is the most reasonable liberal conception of justice, because it best satisfies the conditions for a political conception of justice. ${ }^{31}$ The main reason for this seems to be that the original position is effective in removing the contingencies of particular political situations, and, so, it sets a framework that reasonable individuals are likely to be able to agree to. ${ }^{32}$

\footnotetext{
${ }^{26}$ Ibid, p214.

${ }^{27}$ Ibid.

${ }^{28}$ Ibid, p217.

${ }^{29}$ Ibid, p223.

${ }^{30}$ Ibid.

${ }^{31}$ Ibid, pxlvii.

${ }^{32}$ Ibid, p275.
} 
Rawls' public reason allows citizens to engage democratically in political decision making. They act not to further their comprehensive interests, but to apply their sincere opinions as to the most reasonable political course. ${ }^{33}$ The conditions of reasonableness and the reciprocity that this implies, along with the sincerity of opinion and a unified overlapping consensus, mean that the political result is fair and has been reached not as an expression of the power of one group or belief system, or as a bare compromise between factions, but, rather, as the full political expression of reasonable citizenship. This does not mean that full agreement will necessarily be reached, but, in the context of a reasonable pluralist democratic society, this public reason in the domain of the political is, Rawls argues, the best approach to just government and the legitimate exercise of coercive power. ${ }^{34}$

In general terms, Rawls' system of political liberalism is broadly conceived and ingeniously constructed. It is founded theoretically on the meta-ethical distinction between the political and the philosophical/metaphysical. In the social context, it depends on the fact of reasonable pluralism in modern democratic states, which is informed by this distinction in its practical form, namely between the political and the comprehensive. Rawls argues that, from this reasonable pluralism, we are led inexorably to the possibility of a political conception of justice, which we can all reasonably endorse. This conception is made possible by the overlapping consensus between our comprehensive beliefs, and given substance by the action of a reasonable system of political reason.

\subsection{The political and the true}

The distinction between the political and the metaphysical, and its practical expression, in the distinction between the political and the comprehensive, is a fundamental conception of Political Liberalism. The idea of the political is one of the practical inquiry and expression of a society that is plural, yet democratic and reasonable. This is contrasted, firstly, with the metaphysical or philosophical, which implies a search for an objective and singular truth, and a rationally justified moral

\footnotetext{
${ }^{33}$ Ibid, pplv, 241.

${ }^{34}$ See ibid pp226, 241.
} 
system; and, subsequently, with comprehensive doctrines, which are belief systems that correspond broadly to a particular philosophical position. The latter are historically situated, relatively coherent doctrinal expressions of a philosophical or metaphysical kind. In this sense, comprehensive doctrines are the societal outcome of a philosophical or metaphysical approach, and, so, the second distinction is the sociological counterpart of the first.

This central conception serves both justificatory and structural functions in political liberalism. It allows Rawls to eschew the universal, and, instead, suggests a contextualised approach, contingent on the conditions of modern democratic societies. The outcomes of political liberalism, those constitutional essentials and principles of basic justice, are expressions of agreement between free and reasonable democratic citizens. They are contingent on the particular social context, and Rawls makes it clear that these public expressions can take a various number of forms. He does assert, however, that some forms are better than others, since he believes that justice as fairness is the most reasonable liberal conception.

In many ways this resembles the intuitionism that was part of A Theory of Justice, although, instead of individuals seeking a reflective equilibrium of considered judgments about justice, they are expressing an honest and considered opinion about politics. In effect, Rawls has removed the truth-claims of his theory and acknowledged the reasonable contestability of different liberal forms, and embedded his justice as fairness in a political system that is predicated on disagreement. This shift conveys an acknowledgement of moral contestability and disagreement that was not present in A Theory.

The structural effect of the political-comprehensive distinction is to demarcate a domain within which people have differing (reasonable) comprehensive doctrines, from a domain that is concerned with matters of social justice. It seems that this distinction performs much the same role that the priority of the right over the good does in A Theory. There, the justification of a conception of justice does not depend on a particular theory of the good, but, rather, on fundamental moral concerns, as encompassed by the right. This informs a structure where welfare is subordinated to liberty and equality. In Political Liberalism, justice is independent of the particular 
comprehensive doctrines that individuals and groups accept, and comes, instead, from the common and reasonable political expression of the citizens of a democratic society. Both of these systems define a space of free liberal choice, and both separate this choice from the concerns of justice, founded on either the right or on the political.

The shift from right/good to political/comprehensive is interesting. Firstly, personal conceptions of the good life, or of the best way to live, now recognise not only a personal self-interested choice, but also the importance of the context of this choice, the belief systems and traditions that underlie it. Undoubtedly, this change has occurred because of the deepening of the understanding of the self that developed from and in response to the communitarian criticisms of liberalism.

Probably more startling is the change from the right, a set of universal foundational moral concerns, to the political, which is characterised as more open and contextual, and as being reasonable rather than true. An apparent benefit of this shift away from truth is that the same justificatory stringency should not be required. Even though the content of the political domain is not philosophical or true, this does not mean that Rawls' Political Liberalism functions in the absence of truth. As David Estlund recognises, "[i]t is important to distinguish Rawls's view that the political conception does not require truth, from a comprehensive or "metaphysical" doctrine that truth is not required". ${ }^{35}$ The standard of reasonable acceptance rather than truth may be appropriate for the political domain, but this does not remove the justificatory requirements of the theory itself. This means that, for example, even if the political is not true, the distinction between the political and the comprehensive and its consequences must be true.

Joseph Raz has made this point more forcefully in saying that there are many theories of justice, and they are all acceptable to the same degree as theories of
justice. To recommend one as a theory of justice for our societies is to recommend it as a just
theory of justice, i.e. a true, or reasonable, or valid theory of justice. If it is argued that what
makes it the theory of justice for us is that it is built on an overlapping consensus and
therefore secures stability and unity, then consensus-based stability and unity are values that a

${ }^{35}$ D Estlund, 'The Insularity of the Reasonable: Why Political Liberalism Must Admit the Truth' (1998) 108 Ethics 252, p260. 
theory of justice, for our society, is assumed to depend upon. Their achievement ... makes the theory true, sound, valid, and so forth.... There can be no justice without truth. ${ }^{36}$

Raz argues that the value of a theory of justice cannot be independent of the truth of its claims, and, in particular, the truth of the values that it promotes. A theory of justice may be justified because it respects people, or it achieves equality, or it is agreed to by everyone; but, this must mean that it represents true moral value. Raz argues that political liberalism claims value by securing a consensus-based system that boasts stability and unity; this depends on both the truth of this statement and the implicit judgment that such stability and unity is morally valuable.

Estlund challenges Rawls' theory in a similar way:

$[\mathrm{P}]$ olitical liberalism must assert the truth and not merely the reasonableness ... of its foundational principle that doctrines are admissible as premises in political justification only if they are acceptable to all reasonable citizens. If it were not true, rejection by reasonable citizens would not render any doctrines inadmissible into political justification. The principle could not be saved by being shown to be acceptable to reasonable citizens. That would assume what is in question: that acceptability to reasonable citizens has this moral significance, in truth. $^{37}$

Estlund emphasises that what is important here is not just factual truth, but moral legitimacy, or, in other words, the truth of statements as to moral value. ${ }^{38}$ He argues that the reasonable acceptance criterion cannot be justified simply by its own reasonableness, and that there must be some external normative evaluation. $\mathrm{He}$ suggests that this criterion, as a political doctrine, must also be subject to its own argument for validity. This means that reasonable acceptability must itself be reasonably acceptable to democratic citizens, as well as being morally legitimate. ${ }^{39}$

These two arguments limit Rawls' claim to reasonable agreement over truth, and his aversion to metaphysics. This makes sense in that the political conception is embedded in a theoretical and justificatory structure that claims to be true. This is a point that Rawls goes to no great lengths to make clear. Consequently, all of these implicitly asserted truths and moral values are subject to much the same contestability as are the various aspects of comprehensive liberal theory. In this way, the distinction between the political and the philosophical, and the resulting structure, is potentially

\footnotetext{
${ }^{36} \mathrm{~J}$ Raz, Ethics in the Public Domain: Essays in the morality of law and politics (1994), p55.

${ }^{37}$ Estlund, op cit, above note 35, p253.

${ }^{38}$ Ibid, p274.

${ }^{39}$ Ibid, pp253-254, 268.
} 
subject to the same liberal bias that affects the priority of the right over the good: people are at liberty to live their chosen reasonable doctrines; but, it is the liberal who defines the overarching structure and moral objectives of society, and who sets boundaries on proper action. The necessity of justifying this structure is obscured by its openness and moral plausibility.

The bias manifest in Political Liberalism is not merely liberal, but is political and contextual as well. Its main moral values include agreement, stability, unity and reciprocity, which are not, excluding perhaps the first of these, typical liberal values. The whole justificatory point of political liberalism is that it reaches beyond normal liberal ideals, and is designed to be able to attract the endorsement of all reasonable democratic citizens. It is the feature of reaching beyond the liberal system of beliefs on the one hand, and being open to the actual substance of justice on the other, that represents the attraction of liberalism in this political form.

However, this structure does require explicit justification: the fact of reasonable pluralism in a democratic state must be, in a socio-historical sense, true; the values of stability and unity must have strong universal moral power, since they are logically prior to Rawls' contextual approach, and the system must effectively promote these values; the legitimacy criterion of reasonable agreement must have contextual moral power, contingent on a modern democratic state; and, finally, the foundational dualism of the political and the philosophical must be accepted both meta-ethically, and in its structural form.

\subsection{Reasonable pluralism}

Despite its many facets and complexities, Political Liberalism is, essentially, a theory of liberal pluralism. As such, the central justificatory point is the fact of pluralism. Whereas a typical value-pluralist approach would rest on the pluralism of incommensurable ways of life, Rawls' formulation is of a reasonable pluralism of conflicting comprehensive doctrines. The one substantial difference between these two approaches is Rawls' requirement of reasonableness. 
The first element of this reasonableness is the willingness to accept fair terms of cooperation, given that others are similarly willing. This requirement is a practical expression of the separation of political and comprehensive doctrine: a reasonable person accepts fair terms of cooperation that are independent of their own personal beliefs. In other words, they must accept a clear separation between personal and political lives. This cleaving between personal and public has been described by Will Kymlicka as "ingenious", in that it allows a person to have a (communitarian) personal identity, and, yet, engage in the public (liberal) sphere. ${ }^{40}$ Kymlicka and others also recognise the "rather schizophrenic attitude" this requires. ${ }^{41}$ This is particularly the case for members of communitarian groups, or groups whose comprehensive doctrines do not envisage or allow for this separation of private and public. Political liberalism would require that they adopt this separation in their lives, and that they act politically according to the rules of public reason. There is the potential here for the undermining of personal or group comprehensive doctrines: either, by setting rules as to how one must act politically that are contrary to accepted practices, or, simply because of the potentially disruptive effect of the political on personal life. $^{42}$

In defence of Rawls' position, the overlapping consensus does imply that the core of the political approach is acceptable to all comprehensive conceptions. At the heart of this consensus is a shared democratic culture and beliefs. Given this pre-existing political attitude, it is perhaps realistic for Rawls to impose the requirement of fair cooperation on citizens. The problem with this, however, is that the relationship of our current attitude of political involvement to our personal beliefs is much less clearly defined than in the system Rawls proposes. For example, we are largely free to engage or to ignore political matters as we wish or as our personal beliefs dictate; equally, we are free to determine our political attitude and beliefs by whatever means we choose, and, in particular, can build our political beliefs out of our personal ones. Although there is much of the democratic culture that is useful to Rawls, this requirement imposes a firm model of the way our personal and political lives ought to

\footnotetext{
${ }^{40}$ W Kymlicka, Contemporary Political Philosophy (2 ${ }^{\text {nd }}$ edn., 2002), p236.

${ }^{41}$ S Mulhall \& A Swift, Liberals and Communitarians ( $2^{\text {nd }}$ edn., 1996), p223.

${ }^{42}$ See ibid, p223.
} 
interact that goes far beyond current attitudes, and is a substantial and potentially quite coercive condition placed on acting in a political liberal society.

The second part of the reasonableness of persons concerns the acceptance of the burdens of judgment; that there are particular limitations on judgment that imply the recognition of the fallibility of one's personal beliefs and commitments. ${ }^{43}$ This requirement seems to ensure that individuals, who are committed to reaching fair terms of agreement, are willing to look beyond their own personal understanding of what this might mean, and to engage generally in political reason with an attitude of openness towards the beliefs of themselves and others. As a feature of reasonable citizens, this would tend to make public reason more effective in reaching a political conception of justice, and in finding an overlapping consensus. In relation to their role within the overall theory, the burdens of judgment also emphasise that political liberalism and the marginalisation of truth does not imply a relativism of beliefs: it is not that political claims are merely personal or that no true moral claims exist, but, rather, that our own judgment of these issues is imperfect and fallible.

Leif Wenar argues that the burdens of judgment are an unnecessary and exclusionary condition of the reasonable person: unnecessary because liberal toleration in public reason is secured by the requirement of fair cooperation; and, exclusionary in that they would deem to be unreasonable many who political liberalism should accommodate.

\footnotetext{
The difficulty is that religious doctrines typically deny that the burdens of judgment obtain. This, on reflection, should not be surprising. The burdens of judgment are meant to explain... why some people believe in one faith, while others believe in other faiths, and still other are agnostics and atheists ... By contrast, a religious doctrine - as a purportedly authoritative guide to moral requirements and/or salvation - characteristically presents itself as universally acceptable to clear minds and open hearts. ${ }^{44}$
}

The only possible escape from this argument for the believers of religious doctrine would be to limit the effects of the burdens of judgment to knowledge and belief that is political or is expressed in the political domain. This, however, does not quite make sense: the separation of political and comprehensive is not so complete as to require a person to adopt different attitudes to moral judgment and belief depending on the

\footnotetext{
${ }^{43}$ Boettcher, op cit, above note 16, p605.

${ }^{44}$ Wenar, op cit, above note 16, pp43-44.
} 
domain in which they are acting; and, this separation does not go so far as to require a true believer to be an ethical sceptic once they move from personal to political life.

A defender of the burdens of judgment might claim that this division, and the attitude implied by the burdens, might de derived from the common democratic culture, so that any democratic citizen might be expected to accept the public-private distinction. As argued above, this distinction requires more than western democratic culture entails. It is quite possible to be a religious adherent and believe in participating in democracy without being burdened by a belief in the fallibility of value judgments. Because such people may well be imbued with a democratic spirit and the desire to enter into fair political arrangements, they seem to be valid subjects of a political conception of justice.

Rawls' conception of reasonableness is certainly clever: it includes for consideration only those with a democratic attitude, willing to play down the content of their comprehensive beliefs in order to achieve a cooperative and reciprocal justice. This is the central project of Political Liberalism: to move from conflict in a democratic society to liberalism. Yet, the conditions of reasonableness are too restrictive to incorporate easily the modern democratic attitude, and the separation required between political and comprehensive too sharp. It is almost as if Rawls takes the content of the political-comprehensive distinction too seriously. He seems to try to make a crystallised, logical prescription about our attitude and approach to our public lives out of a distinction that is under-defined and amorphous, and that cannot possibly bear the weight that Rawls tries to place on it. Even the underlying theoretical conceptions of the political and the metaphysical are not delimited sufficiently. The similarities between these problems, and those of the priority of the right over the good, are considerable.

The reason why Rawls should want to place such close and potentially questionable restrictions on the reasonable is clear: the more restricted is the reasonable, the easier it is to find an overlapping consensus, and to achieve the reasonable agreement that links pluralism and liberalism. Unfortunately, as the reasonable is restricted and becomes less realistic, it moves further and further away from a recognisably modern and inclusive democratic attitude towards justice. This restrictive strategy is 
interesting in contrast to value-pluralism: Rawls is not trying to argue from the fact of pluralism to liberalism, and, in the process, justify liberal restrictions on value conflicts. Instead, he identifies the fact of reasonable pluralism as his starting-point, and, in so doing, excludes a great deal of potential pluralist conflict.

In a sense, Rawls has not identified a bare pluralism, but rather a democratic pluralism, within which lies the prospect of liberal redemption. This makes the link between pluralism and liberalism much more readily achievable, but it means an additional justificatory burden. In particular, Rawls needs to explain why only those who are reasonable are relevant to his conception of political justice, and why the unreasonable are excluded. The validity of the agreement on justice that is reached depends on adequately explaining the exclusion of many from this agreement. This exclusion of the unreasonable is a difficult aspect of Political Liberalism, and one that Rawls never really answers. By implication, such people are excluded from public reason, and are forced to adhere to the political conception that is reached; yet this position appears neither democratic nor liberal. ${ }^{45}$ They would presumably enjoy the same liberties and benefits as would any citizen, but would have no role in determining what these were.

In the justificatory sphere, it is important for Rawls to have a definition of reasonable that is as broad as possible, to give reasonable agreement as much moral power as possible, so that it is not simply a case of a group of liberal citizens getting together and agreeing on a liberal conception of justice. ${ }^{46}$ A reformulation of these requirements might be able to overcome many of the problems described here. This might involve a number of changes: the first of which would be a restatement of the distinction between political and comprehensive that gives effect to the basic idea that citizens should conceive a division between their own beliefs and what they are willing to accept and support as a common idea of justice, without amounting to an unrealistic schizophrenia. The positive attitude towards fair and reciprocal cooperation would be a central requirement, and would mean an acceptance of the need to downplay one's own beliefs, and to look for common ground from which to

\footnotetext{
${ }^{45}$ See M Friedman, 'John Rawls and the Political Coercion of Unreasonable People' in V Davion \& C Wolf (eds.), The Idea of a Political Liberalism: essays on Rawls (2000), pp22-23.

${ }^{46}$ See, for example, ibid, p28.
} 
express and discuss justice in a way that could be accepted by those with different beliefs. The burdens of judgment would be removed altogether, as contemplated by Wenar, and perhaps replaced by an acceptance of the problems involved in imposing uncommonly held beliefs on others. This would be joined by a belief in the basic constitutional ideas of the rule of law and the separation of powers. Such a reformulation of the reasonable might be enough to correct some of the shortcomings of Rawls' conception, and provide a foundation for a more workable form of political liberalism.

\subsection{From reasonable pluralism to political liberalism}

As suggested in the previous chapter, liberal pluralism needs to define and justify a strong link between pluralism and liberalism that takes advantages of the affinities between the two. With political liberalism the link consists of agreement in the political domain, but this needs to be read in the context of Rawls' restriction of pluralism to the reasonable.

Because liberalism is not the only or the necessary response to pluralism, the nature of the response of a particular liberal theory needs to be examined and justified. Rawls does not undertake this task, and relies simply on the value of agreement and consensus, and the consequential values of stability and unity. Charles Larmore, another political liberal, goes further in identifying its main value and justification.

Political liberalism, as I conceive it, aims, of course, to forgo appeal to any comprehensive conception of the human good. Its ambition consists in working out principles of political association that citizens can see reason to accept, despite their inevitable differences concerning ultimate ends. All the same, this ambition draws upon an underlying view of human dignity and the respect that we thereby owe each and every human being. ${ }^{47}$

Larmore perceives human dignity and respect as the values that underlie the criterion of reasonable agreement. He goes on to explain the nature of the respect involved.

Persons are beings capable of thinking and acting on the basis of reasons. If we try to bring about conformity to rules of conduct solely by the threat of force, we shall be treating persons merely as means, as objects of coercion, and not as ends, engaging directly in their distinctive capacity as persons. True, they cannot be moved by threats except by seeing that they have

${ }^{47}$ C E Larmore, 'Respect for Persons' (2005) 7.2 The Hedgehog Review 66, p69. 
good reason to fear what we may otherwise do. But we shall then be appealing to their ability to act on reasons simply in order to achieve the goals of compliance.... We shall not be engaging their distinctive capacity as persons in the same way we engage our own, making the acceptability of the rules in question depend on their reason just as we believe it draws upon our own. Thus, to respect another person as an end is to require that coercive - that is, political, principles be as justifiable to that person as they presumably are to us. ${ }^{48}$

The significance of this respect for our capacity to make moral judgments and to reason, Larmore argues, is not that reasonable people share it, but rather that it is

what directs us in the first place to seek our principles of our political life in the area of reasonable agreement. Respect for persons lies at the heart of liberal thought, not because looking for a common ground we find it there, but because it is what impels us to look for a common ground at all. ${ }^{49}$

From this we can map out a plan of the justification of Larmore's version of liberalism. At its heart is the criterion of reasonable acceptance, which is valued because it reflects an equal respect for people. This respect is based on our individual capacity to think and act for reasons, and requires that we be treated as ends and not means, and not be coerced at the will of another. This unmistakably deontological respect is Larmore's foundational value of political liberalism. Its value is not derived from the operation of public reason or a liberal system, but, rather, is external to and, in fact, implies such a system.

It is clear that this respect is not drawn from, but is morally separate and prior to, the fact of pluralism. It is the value of this respect superimposed on a pluralist foundation that leads Larmore to the liberal value of public justification and reason. The danger with such an approach, as the preceding chapter suggests, is that this respect is subject to the challenge that it is merely one among plural values, and cannot rationally be shown to be better than any other, except in contravention of pluralism. In order to avoid this result Larmore's political liberalism must either use a different conception of pluralism or must explain and justify the additions grafted onto the simple structure of pluralism-liberalism.

As it turns out, this form of liberalism implies a number of key differences from the thoroughgoing value-pluralism of Galston. Firstly, the pluralism of political liberalism does not indicate a fundamental philosophical truth. Instead, it is treated by Rawls

\footnotetext{
${ }^{48}$ Ibid, pp71-72.

${ }^{49}$ Ibid, p72.
} 
more as a contextual socio-empirical fact: that, in society there is a diversity of incommensurable doctrines, traditions and conceptions of the good. This form of pluralism does not necessarily undermine the claim of a particular value to special moral importance, although it does emphasise that this value must be justified in a way so as to transcend the conflict and social dialogue between doctrines. The value of respect has considerable appeal, in that it acts in relation to the very capacities that allow us to create and subscribe to these doctrines. Despite the apparent value of respect, and the fact that there is no logical contradiction between it and reasonable pluralism, the value of respect is still in need of careful explanation and justification.

Another interesting point is that public reason in political liberalism depends on a common political (democratic) culture, and aims at reaching an overlapping consensus that all reasonable people can accept regardless of their comprehensive beliefs. This focus on common beliefs and understandings has the potential to work against a foundation of value-pluralism, and to promote particular liberal values. This would mean that respect might be justified in having a particular role despite the incommensurability of values, by virtue of being part of what is shared between people. This result depends on something being shared, but nothing in the idea of a plurality of values prevents a particular value being held by many or all people; in a system where a common public culture is assumed, this is a real possibility.

Such an argument would result in a value that is justified in a particular social context, and would overcome a foundation of value-pluralism. However, this is not the approach to respect that Larmore intends. His comments assert that respect is more fundamental than being merely a political virtue that reasonable people hold: it is not the common ground, but is what impels us to look for common ground. This suggests some moral content to Larmore's conception of respect that is separate from the fact of pluralism and from democratic culture; and, that Larmore is not simply using respect to mediate between pluralism and liberalism, but rather to draw from a deep universal moral truth. This is confirmed by comments Larmore makes about pluralism elsewhere:

Liberalism ... does not arise from an acceptance of pluralism. Instead, it seeks to found the principles of political association upon a core of morality that reasonable people can accept, despite their natural tendency to disagree about comprehensive visions of the nature of value, 
and so in particular about the merits of monism and pluralism. The expectation of reasonable disagreement lies at a different, more reflective, level than pluralism. It responds to the religious and metaphysical disenchantment of the world, not by affirming it, as pluralism seeks to do, but rather by recognizing that like other deep conceptions of value this disenchantment is an idea about which reasonable people are likely to disagree, as indeed they do. ${ }^{50}$

Larmore here sees political liberalism not as an appropriate response to pluralism, but as having a core morality of its own; it is clear that respect would form a major part of this core. His rejection of pluralism as a foundation for liberalism is interesting. He conceives pluralism, not as a foundational fact either about moral value or actual belief, but, rather, as itself a questionable view of morality that is capable of being the subject of his preferred conception of moral conflict: reasonable disagreement. So, pluralism does not even enjoy the status of being socially common, let alone that of a fundamental moral truth. Consequently, it becomes, itself, the subject of reasonable disagreement. He goes on to describe how pluralism affirms philosophical disenchantment, conceiving of it in terms of the political ideal of promoting a variety of beliefs.

It appears here that Larmore conflates the idea that moral value is irreducibly plural in nature with the prescription that society should promote a diversity of beliefs; of pluralism as a moral truth with pluralism as a positive ideal. He then treats the resulting conception of pluralism as a possible moral choice, which is consistent with it containing substantive moral content, and he substitutes reasonable disagreement for pluralism. What remains is a liberalism based on reasonable disagreement, or, in other words, on the acknowledgement of moral contestability between reasonable people. The implication is that moral value is not necessarily singular or plural, but that there is disagreement about it.

There is nothing substantially different between this view of reasonable disagreement and the reasonable pluralism that is the more obvious focus of Rawls. The point for Larmore seems to be to distinguish his liberalism from what he views as the necessary prescriptions that attach to value-pluralism. It is interesting, however, how easily the fervently held belief of difference of one theorist becomes a subject of difference of another: how pluralism becomes another value about which we can so readily

${ }^{50}$ C E Larmore, The Morals of Modernity (1996), p165. 
disagree. It is interesting to speculate whether Larmore's conception of reasonable disagreement could be so treated by someone else: whether disagreement is the true shape of social interaction, or merely one of many possible outcomes between which no theory can rationally decide. The feature essential to these conceptions is an insoluble contestability between conflicting positions.

There is the vague suggestion here that no single account of persistent difference, be it expressed as disagreement or pluralism, can represent the final truth about difference, as any account could be the valid subject of a separate account of broader difference. The nature of this difficulty is the tension between the complexity of difference and the idea of being able to reduce and express this difference in a singular, unified way. This is a disconcerting and potentially decisive comment on the possibility of certainty, but, for the present purposes, it is enough to note that it suggests that liberalism cannot rely simply on an account of pluralism to count as an effective response to moral uncertainty.

In whatever form this difference is presented, it is used cleverly by political liberalism, and does seem to be capable of overcoming the inconsistency between pluralism and liberalism explored in the previous chapter. This is achieved by the expression of pluralism as reasonable pluralism, and, so, by restricting the domain of irreducible moral disagreement to the reasonable. There is no necessary conflict between this pluralism and the values that political liberalism express, since the former does not comment on moral value generally, and makes a categorical distinction between this pluralism, which is related to comprehensive doctrine, and the political anyway.

More generally, political liberalism works from the fact of reasonable pluralism, without its own principles becoming the subject of this pluralism. This is achieved by virtue of the unifying nature of this disagreement, and of the restriction of reasonableness placed on it. Instead of working from a population of people who reflect the basic moral truth of value-pluralism, political liberalism is founded on a population of democratic individuals who recognise the presence of persistent disagreement, and are willing to agree on fair terms of cooperation, which need not accord entirely with their own beliefs. 


\subsection{Overlapping consensus}

The overlapping consensus between reasonable comprehensive doctrines is important to Rawls' theory as it utilises our common political culture to steer political liberalism away from a modus vivendi, a mere accommodation made on pragmatic grounds. Instead, the political conception of justice is supposed to be agreed to for the right reasons: founded on our common understandings of justice, but mediated by our commitment to fair terms of cooperation and our realisation that the political domain cannot be simply the vehicle for the promotion of our own comprehensive beliefs. As Patrick Neal has commented, political liberalism is supposed to be a compromise between a prudent, morality-free modus vivendi, and an unreservedly moral promotion of our comprehensive beliefs, so as to allow "not too little, not too much, just the right amount of morality into the terms of political order". ${ }^{51}$

It is supposed to offer a democratic society political unity and stability, despite the plurality of conflicting beliefs. This depends, however, on whether such an overlapping consensus would actually exist, or, in other words, whether there really would be this common fund of foundational political beliefs from which political liberalism might draw. Neal suggests that the possibility of an overlapping consensus is not possible as "political disagreement cannot be stopped at the edge of political liberalism, because that abstract idea is itself open to numerous competing interpretations". ${ }^{52}$ The contestability of the system of political liberalism itself, he argues, and, in particular, the criterion of reasonable agreement, would prevent the kind of consensus that political liberalism anticipates; people would never agree to the terms of an overlapping consensus, let alone to its content.

Even if a democratic society could agree to implement that system, there are others who believe that an overlapping consensus would still lack content. James Bohman cites the deep cultural conflicts within modern democracies that challenge the "basic

\footnotetext{
${ }^{51}$ P Neal, 'Three readings of political liberalism: Rawls, Maritain and Crick' (2000) 5(2) Journal of Political Ideologies 225, p242, inverted commas omitted.

${ }^{52}$ Ibid, p243.
} 
framework of moral assumptions and political procedures", in arguing that such a consensus would be unavailable: ${ }^{53}$

If we accept the social facts of pluralism and deep conflict, then we must also wonder whether the scope of what is "reasonable for all to accept" turns out to be so small as to be irrelevant for most political disagreements. ${ }^{54}$

Rawls would probably reply that such a deep conflict would imply that at least one of the conflicting parties is not actually reasonable, in that they, either, do not accept the requirements of reasonableness, or, they do not share with the other parties a common democratic political culture. It is difficult to predict the extent of an actual consensus, but these restrictions on the parties have led Stephen Mulhall and Adam Swift to conclude the opposite, that an overlapping consensus is actually guaranteed:

[I]f part of what it means for a comprehensive doctrine to be reasonable is that it accepts the normal priority of the political over the non-political, then there can be no need to seek an overlapping consensus between them, since their reasonableness simply guarantees it by definition. $^{55}$

Mulhall and Swift focus on the reasonableness of doctrines rather than people, and identify the priority of the political as being sufficient from which to infer some form of consensus. Perhaps this consensus is not so full as to include all the culture-based fundamental ideas of justice that Rawls anticipates, but it does at least set the foundation for these.

The existence of an overlapping consensus depends closely on the restrictions on reasonableness, since these specify what is necessarily common between the relevant citizens. Against these is the social fact of a plurality of beliefs. Social conflict might well be deep in the sense that Bohman suggests, but whether this can occur among the reasonable, and how such conflict would play out in the political domain, is unclear. There is at least the foundation for a political consensus based on the democratic public culture of a society. Perhaps Bohman has underestimated the role of this culture, and the necessary similarity of perspective that it implies.

\footnotetext{
${ }^{53}$ J Bohman, 'Public Reason and Cultural Pluralism: Political Liberalism and the Problem of Moral Conflict' (1995) 23(3) Political Theory 253, p254.

${ }^{54}$ Ibid, p255.

${ }^{55}$ Mulhall \&* Swift, op cit, above note 41, p241.
} 
Rawls' use of an overlapping consensus to lay the foundation for a political conception of justice, rather that a modus vivendi, is clever; but, he seems to ask a great deal of the political commonalities between people, if they are to accept in political argument his system of justice as fairness, as well as the two resulting principles, as the most reasonable possible conception. To achieve these results Rawls has to narrow the idea of reasonableness, and place significant weight on democratic political culture, to the point that one wonders if any resulting overlapping consensus is merely a reflection of the narrowing of democratic society that Rawls undertakes.

\subsection{Public reason}

It is the realm of public reason in which the political arguments are presented to legitimise coercive state behaviour, and to justify the state preferring the claims of one person to those of another. The scope of this purpose is defined by Rawls as including constitutional essentials and matters of basic justice, and is supposed to be made up of content upon which a broad range of reasonable persons can agree.

The use of public reason as the process by which the substance of a theory of justice is reached represents an important shift in liberal theory. Earlier systems included the structure and justification of a particular substantive approach to a theory of justice, whereas political liberalism recognises plural comprehensive liberalisms and declines (initially at least) to choose between them. In this way, the principles of justice a society is to follow are no longer the result of a hypothetical and carefully constrained situation, but, rather, part of a political process of justifying state coercion to those who will be coerced.

This is a subtle shift, but potentially a powerful one. By reserving questions of substantive justice to operation of actual public reason, it is possible to relocate within that discourse many of the difficulties that otherwise would be present in a theoretical structure. In relation to Rawls' justice as fairness, for example, the contestability concerning the difference principle, and the priority of the liberties over the good, could be left to actual political discourse. In the context of reasonable democratic 
citizens, contestability over the substance of justice is inevitable, and forms part of an open forum of public reason. Once these contestable issues have been excised, a liberal theory can focus on searching for matters of justice that are more fundamental and less contestable.

In this way, public reason can also remove much of the complexity necessary to complete theories of justice. By allowing the more substantive matters to be determined by the operation of the political processes implied by a theory, rather than simply declared as part of its structure, much of the complexity of these substantive issues is shifted to actual political processes. In addition, a liberal theory that incorporates public reason can better incorporate actual moral difference, rather than simply difference as to conceptions of the good, without this undermining liberalism itself. This difference can form a legitimate part of the process by which principles of justice are determined. In this sense, public reason allows liberalism to be more open to substantive matters of justice; it also allows liberalism to focus on more fundamental philosophical or structural issues. This is a potentially important liberal idea, but the question remains as to how successfully Rawls employs it as part of his political liberalism. The difficulty in distinguishing what is constitutionally essential to the structure of basic justice from what is not is clearly problematic and, as has been recognised, Rawls makes no attempt to explain this. ${ }^{56}$ However, this limitation is probably more pragmatic than principled; and, it seems to result from Rawls wanting to start with a subject that is most obviously political and where he is most likely to find accord. ${ }^{57}$

A more difficult aspect of Rawls' public reason is the distinction between those reasons that are acceptable and those that are not, and whether the domain of the political is sufficiently encompassing to allow a coherent result. It is interesting here that Political Liberalism itself is reluctant to allow any of a person's background or non-political culture to be expressed as part of public reason. The basis of Rawls' argument is that political values present viable limits for the fundamental political questions, and that non-public reason is separate, and is itself the subject of liberties

\footnotetext{
${ }^{56}$ Ibid, p225.

${ }^{57}$ Rawls, op cit, above note 10, p215; see also Mulhall \& Swift, op cit, above note 41, p227.
} 
granted by public reason. ${ }^{58}$ In a later essay, 'The Idea of Public Reason Revisited', he allows that comprehensive doctrine can be introduced into public reason "provided that, in due course, we give properly public reasons to support the principles and policies our comprehensive doctrine is said to support". ${ }^{59}$ This proviso must be satisfied according to the "good sense and understanding" of the political culture involved. ${ }^{60}$

The reason for Rawls' minimising or excluding non-public content in public reason is clear: public reason is supposed to express a political culture, and to lead to results with which all reasonable people can agree. This stems ultimately from the antiperfectionist instinct to exclude personal beliefs and conceptions of the good from the determination of matters of justice. Yet, in this later essay, Rawls concedes the necessity of comprehensive doctrines as part of what he labels a wide view of public political culture. The reason he offers for this is that

[c]itizens' mutual knowledge of one another's religious and nonreligious doctrines ... recognizes that the roots of democratic citizens' allegiance to their political conceptions lie in their respective comprehensive doctrines, both religious and nonreligious. In this way citizens' allegiance to the democratic ideal of public reason is strengthened for the right reasons. ${ }^{61}$

The meaning of this passage is not straightforward, but it seems to make two arguments. The first is that the roots of allegiance to political conceptions are comprehensive, or, in other words, the reasons why a person adopts a particular political conception relates to their personal comprehensive doctrine. This is entirely plausible: it makes sense that our political beliefs would be related to our comprehensive beliefs, properly constrained by the meaning of the political and our reasonableness.

The second argument is that the sharing of the relationship between comprehensive and political beliefs would strengthen personal allegiance to the process of public reason. Rawls' reasoning seems to be that, when those who follow a particular comprehensive doctrine adopt the proviso, they show to others their commitment to

\footnotetext{
${ }^{58}$ Rawls, op cit, above note 10, pp215, 220-222.

${ }^{59}$ Freeman (ed), op cit, above note 1, p584.

${ }^{60}$ Ibid, p592.

${ }^{61}$ Ibid.
} 
public reason. ${ }^{62}$ This fosters civility and reciprocity as features of the public process, not for pragmatic reasons, but because they understand the reasoning of the other party from comprehensive to political belief, and respect their commitment to supplying political rather than comprehensive reasons. In this way, the proviso, Rawls argues, is valuable because adherence to it encourages others to engage properly in the public reason process. Comprehensive beliefs, then, are a valid but provisional part of public reason, because they demonstrate that a person or group is committed to genuinely engaging in the public reason process, and in building a political conception capable of being the subject of reasonable agreement, rather than simply expressing their own comprehensive doctrine in the public domain.

It is eminently plausible to say that a mutual understanding will deepen and enrich a process of public reason, but Rawls' argument does not quite work in the way that he suggests. There will also be benefits to public reason of knowing that the other parties are acting genuinely and positively, but this does not help Rawls much either. The problem with his argument for the proviso is that, if there is a real distinction between a political conception and a comprehensive doctrine, people ought to be able to recognise that; an explanation from the other party, as to how the political belief is founded in a comprehensive doctrine should not make any difference to this. The very nature of a political conception is its lack of commitment, so far as is possible, to any comprehensive doctrine. ${ }^{63}$ It is this that makes it agreeable to individuals with diverse comprehensive, although reasonable, beliefs. In this way, political conceptions are, by definition, recognisable to reasonable people. No explanations by others to explain them, from the perspective of their own comprehensive beliefs, should be required.

The proviso itself does not satisfy this justification anyway: it anticipates positive comprehensive arguments being used to justify aspects of a conception of justice, rather than comprehensive explanations and understandings of essentially political arguments. Overall, Rawls' explanation of the role of comprehensive beliefs in public reason is difficult to reconcile with his conception of public reason generally. It seems to serve no useful role in the process; and, the argument that mutual understanding of comprehensive doctrines encourages adherence to public reason does not fit well with

\footnotetext{
${ }^{62}$ Ibid, pp592-593.

${ }^{63}$ Rawls, op cit, above note 10, pp12-13.
} 
either the proviso or the remainder of Rawls' theory, which suggests that reasonable people look to the content of a conception of justice, not to other people's comprehensive arguments in its favour, and that reasonable people would approach public reason for the right reasons anyway. The poor fit between Rawls' political liberalism and his arguments for comprehensive doctrine in public reason suggests that there are other explanations behind the latter. In particular, it raises the possibility that comprehensive doctrine is included because Rawls' political reason in a democratic state is not in itself sufficient to ground a political conception of justice, and additional normative substance is required for any kind of reasonably agreeable content to result.

The actual basis for public reason is broad:

Public reasoning aims for public justification. We appeal to political conceptions of justice, and to ascertainable evidence and facts open to public view, in order to reach conclusions about what we think are the most reasonable political institutions and policies. ${ }^{64}$

This process works from the political culture of a democratic society, which "comprises the political institutions of a constitutional regime and the public traditions of their interpretation ... as well as historic texts and documents that are common knowledge". ${ }^{65}$ From this foundation, through the process of public reason, Rawls asserts that we can reach an overlapping consensus founded on justice as fairness and his two principles of justice. ${ }^{66}$

But, there is good reason to believe that, even among reasonable democrats, people who have accepted the public reason process and search for the most reasonable political conception, substantial contestability would remain. Rawls' suggestion that the political, reasonable and democratic restrictions would lead public reasoning to home in on justice and fairness, or any particular moral result, is hopelessly optimistic given the extent of difference and contestability in the individual comprehensive doctrines on which people's political beliefs are based. If, as Rawls admits, our political beliefs are founded on and formed out of our comprehensive moral beliefs, then the constraints on public reason would have to negate these in order to arrive at a

\footnotetext{
${ }^{64}$ Freeman (ed), op cit, above note 1, pp593-594.

${ }^{65}$ Rawls, op cit, above note 10, pp13-14.

${ }^{66}$ Ibid, pp14-15.
} 
single basic conception of justice. Although Rawls' scheme might allow some constitutional agreement, implied by the reasonable and democratic restrictions, a conception of basic justice would be unreachable, as these restrictions fail to distinguish between opposing reasonable conceptions and ideas.

In this light, including the comprehensive in public reasoning appears, either, as a misguided attempt to incorporate additional substantive moral content, or, as the basis for continuing political disagreement between reasonable people. This argument confirms that Rawls' quest for an overlapping moral consensus on matters of basic justice is flawed and is not reasonably attainable. The idea of public reason as part of a liberal theory is an interesting and potentially powerful one, in terms of relocating contestability and complexity to subsequent public discussion. Rawls' use of public reason, however, is bound up with his conception of reasonableness, the distinction between the political and the comprehensive, and is intended to reach the conclusion of justice and fairness. It is so moulded by the other aspects of Rawls' theory, that it does not make the best of these potential virtues.

\subsection{Conclusion}

Rawls' Political Liberalism is more than a reframing of his two principles of justice with a new focus on stability; it marks a fundamental shift in the approach to liberal theory. At its centre it employs a conception of pluralism, but, unlike the theories of value-pluralism in the previous chapter, uses it in a way that is quite different. Rawls' theory is not concerned with moral truth or with comprehensive moral doctrines, but, rather, with political decisions made by reasonable people, characterised by adherence to a democratic public culture, the willingness to cooperate in the political sphere on fair terms, and the acceptance of various limitations on public judgment. This relocation of liberalism to the political and away from the comprehensive accords citizens the opportunity to engage in a process of determining the most reasonable political conceptions; and, is achieved through a process of public reason, which requires that coercive state behaviour be justified to those whom it affects. 
This approach is explicitly contextual, and applies to the reasonable citizens of modern democratic states. In a way, the change in approach from the seemingly universal goals of A Theory of Justice amounts to an admission of the difficulty of satisfying a universal justificatory standard. It seems to accept that the earlier liberal theories are comprehensive in an important sense, that, although plausible and attractive they arise from a liberal standpoint, and make particular and apparently innocuous statements in what has proved to be a thoroughly contestable moral space.

In admitting the bias of liberal theories, Rawls is in a position to create a theory that can reach beyond existing liberal beliefs, and political liberalism aims at exactly this. It attempts to derive liberal principles not from human nature or moral nature, but, instead, from democratic reasonableness. This is the justificatory heart of Rawls' political liberalism: that reasonable, democratic individuals would agree to a liberal system of public reason as a response to the social fact of reasonable disagreement. The power of this assertion is that it doesn't apply to all people, and that it accepts that real disagreement will remain. What it means is that these people would accept that it is better to have a liberal political system than to work to promote their own comprehensive beliefs in the public sphere. This message implies the acceptance of continuing personal and moral disagreement.

This approach appears to have potential power, depending as it does on the veracity of the claim that reasonable people would accept the political liberal approach. Rawls' conception and structure, however, raises a number of difficulties. His distinction between the political and the philosophical appears misdirected, along with his attempt to eschew the concern of truth from his theory. An effective form of political liberalism may well involve the setting aside of truth considerations in relation to personal belief, but this cannot remove the importance of an effective and true philosophical structure.

Rawls' distinction between the political and the comprehensive is also difficult. Political liberalism depends on effectively distinguishing between the public and private spheres in a way that is acceptable to reasonable people. However, it is difficult to separate those matters that are properly the subjects of political debate from those that are not, and this will be a problem that perhaps no conception of 
political liberalism will be able to overcome. Not only is individual disagreement a problem, but also the natural overlap of personal and political considerations, beliefs and reasons.

One possible approach would be to determine the boundary between the public and the private through the process of public reason; the first step of the making of a political decision would be to determine whether the political domain has jurisdiction to address the matter. However, this result would simply seem to shift the uncertainty about the boundary to the political sphere, and offer no real direction to how these issues should be determined. A procedure for achieving this could be set out beforehand, but this does not solve the problem: to the extent that this offers substantive direction, it characterises the boundary beforehand and so suffers from the problem of disagreement; to the extent that the substance is determined by subsequent public reason, the uncertainty would remain. Combining the two problems together does not manage to solve either.

Along with the distinction between the political and the comprehensive/philosophical, the foundational conception of Rawls' political liberalism is that of reasonableness. This conception amounts to a trade-off: the broader it is, the more powerful is the resulting theory, since it links liberal public reason to a broader base of individuals. The broader it is, however, the less likely it is that the group defined will accept the liberal conclusions, and find an overlapping consensus through public reason. Rawls' reasonableness is disturbingly narrow. The condition of fair cooperation is realistic and broad, but the burdens of judgment imply specific beliefs about the reality of judgment, and seem to exclude those who, presumably, Rawls would want to include. It would be more realistic for him to remove these burdens, but, then, the guarantee of an overlapping consensus, and of the acceptance of justice as fairness as the most reasonable political conception, would be on much shakier ground.

The problems with these foundational conceptions of political liberalism are manifested in Rawls' conceptions of an overlapping consensus, and of public reason. Whether any overlapping consensus would actually result is difficult to judge from Rawls' assertions, but, if so, it would probably simply reflect the narrow characterisation of reasonableness, and the subset of the overall population that this 
applies to. The problem with the distinction between the political and the comprehensive informs the difficulties with public reason, and, in particular, with Rawls' desire to have comprehensive reasons included in public reasoning. Finally, this process is pre-empted by Rawls in his conclusion that his conception of justice as fairness would result as the most reasonable of comprehensive liberal conceptions.

Overall, Political Liberalism represents an ingenious attempt at overcoming many of the problems of earlier liberal theory. As a response to communitarian criticisms, Rawls eschews the philosophical and the true in favour of a political view of justice. This not so much answers as it sidesteps them, by adopting an approach that conceives of moral theory differently than the earlier approaches. But, much like $A$ Theory of Justice, Political Liberalism fails on examination. This is not to deny its significance as a thread of contemporary liberal theory. Political Liberalism is an important attempt at liberal justification, and contains many ingenious and potentially powerful parts: the separation of the political and the philosophical on one side, and the political and comprehensive on the other; the contextualised attempt at justifying the link between pluralism and liberalism; the use of reasonable political pluralism to overcome many of the problems of a thoroughgoing value-pluralism; the assertion of an overlapping consensus based on a public political culture, to overcome a modus vivendi result; and, the use of public reason that deals with the contestability and complexity of substantive political reasoning within a non-theoretical process.

\section{Patterns of Moral Complexity}

Larmore's version of political liberalism has already been discussed in relation to the role of pluralism or disagreement and the virtue of respect, but the theory as a whole features a number of interesting differences from that of Rawls. In fact, Larmore's Patterns of Moral Complexity was published prior to Political Liberalism, in 1987. ${ }^{67}$ Although Larmore did not adopt the label until later, Patterns adopts the two essential features of political liberalism: the distinction between the political and the personal,

\footnotetext{
${ }^{67}$ Of course, it is worth noting that this was subsequent to some of Rawls' first politically liberal essays, including 'Justice as Fairness: Political not Metaphysical',
} 
where liberal principles attach to the former but not the latter, and the use of public reason to respond to or overcome disagreement in the political sphere.

Patterns explores various influential works of political and moral philosophy and suggests that typical approaches to theory tend to simplify their moral subject in three separate ways. These three ignored moral complexities act as the philosophical foundations on which Larmore's conception of political liberalism is built. The first of these emphasises the indispensability of moral judgment, as opposed to simply following a principle or virtue. ${ }^{68}$ Moral judgment helps us in two practical ways: it allows us to recognise that a particular virtue applies to a given situation, and tells us what is required for the virtue to be fulfilled. ${ }^{69}$ Additionally, this judgment assists us in dealing with moral conflicts and dilemmas, rather than relying on the belief that, in such situations, there is some higher-level principle to which we can refer. ${ }^{70}$ Finally, Larmore comments that moral judgment cannot be reduced to some general account or theory. ${ }^{71}$ This conclusion expresses the idea that judgment is by nature not reducible to a rational and concrete structure, and is consistent with his general approach to judgment as a moral complexity.

The second moral complexity concerns the different spheres or domains within which ethics operates. Ethical systems should, Larmore argues, relate to their particular spheres. ${ }^{72}$ He suggests, particularly, that predictability carries considerable weight in the political sphere, because individuals make decisions and take actions in relation to how they believe the state will act. ${ }^{73}$ This places a burden on governments to act in a systematic way, rather than being sensitive to the subtle nuances of every situation, and paves the way for a distinction between the public and private spheres that is necessary to political liberalism. He defines this distinction in terms of "what belongs within the political system" and "whatever belongs outside it."74

\footnotetext{
${ }^{68}$ C E Larmore, Patterns of Moral Complexity, (Cambridge: Cambridge University Press, 1987), p5.

${ }^{69}$ Ibid, p6.

${ }^{70}$ Ibid, p10.

${ }^{71}$ Ibid, pp19-20.

72 Ibid, p40.

${ }^{73}$ Ibid, pp40-41.

${ }^{74}$ Ibid, p42.
} 
The final complexity asserts the heterogeneity of morality. In particular, if we attempt to apply practical reason to alternative situations in order to arrive at some idea of which is more morally valuable, there seems to be three relevant but different general principles, namely partiality, consequentialism and deontology. ${ }^{75}$ Partiality encompasses the moral duties owed by virtue of a person's participation in some institution or association, such as are implicated in relationships between family members or friends. Consequentialism and deontology are familiar to liberal theory, the first maximizing the good, and the second requiring that certain actions should never be done. ${ }^{76}$ Larmore argues that these three principles are all morally significant; that they will often be in conflict; and, that, in the private domain at least, there is no general or straightforward approach to determining which should prevail.

Each of these three complexities acts to support Larmore's conception of political liberalism. The importance of judgment in morality encourages a practical rather than theoretical approach to substantive moral questions, and, ultimately, supports a conception of public reason as the best approach to answering questions of political morality. By asserting the relevance of different moral domains, Larmore clearly paves the way for distinguishing between the public and the private. Finally, the heterogeneity of morality highlights, at the most abstract level, the difficulties of reaching definite and agreeable moral decisions, without reverting to a strict valuepluralism: it helps to explain moral disagreement; but, with the use of the second complexity, does allow Larmore to simplify political morality while accepting the complexity of private morality. In fact, this is the general approach of Patterns: that the complexities of morality can best be dealt with by separating the political and by applying particular knowable rules to that domain, all while retaining the power of public reason as the tool for reaching morally acceptable outcomes.

In order to justify the functioning of the political domain, and to determine the proper relationship between partiality, consequentialism and deontology in the political, Larmore uses a conception of neutrality. Neutrality, he argues, is the best response to pluralism and reasonable disagreement in society. ${ }^{77}$ This means that state decisions

\footnotetext{
${ }^{75}$ Ibid, pp131-132.

${ }^{76}$ Ibid, p132.

${ }^{77}$ Ibid, p43.
} 
must be neutrally justifiable. ${ }^{78}$ One example of a neutrally justifiable goal is that of economic efficiency. Western democracies, Larmore argues, have long pursued economic efficiency as a goal, which means that they are justified in regulating and intervening in economic activity. ${ }^{79}$ Larmore's neutrality extends also to the realm of public reason: if people disagree, then neutrality suggests that they should each retreat to a position of common ground, and continue to converse. ${ }^{80}$

Larmore is then tasked with justifying this conception of neutrality. Traditional liberal justifications fail, as they depend on particular conceptions about human good that people may reasonably dispute, for example, neutrality based on the value of autonomy. ${ }^{81}$ Larmore suggests, instead, a justification suitable for a political approach to neutrality: a neutral justification for political neutrality, one which avoids contestable conceptions of the good, by itself, being neutral between such conceptions. ${ }^{82}$ This neutrality is based on what Larmore calls the universal norm of rational dialogue:

\begin{abstract}
When two people disagree about some specific point, but wish to continue talking about the more general problem they wish to solve, each should prescind from the beliefs that the other rejects, (1) in order to construct an argument on the basis of his other beliefs that will convince the other of the truth of the disputed belief, or (2) in order to shift to another aspect of the problem, where the possibilities of agreement seem greater. In the face of disagreement, those who wish to continue the conversation should retreat to neutral ground, with the hope either of resolving the dispute or of bypassing it. ${ }^{83}$
\end{abstract}

He accepts that this is not morally neutral, as it depends on a moral commitment to rational dialogue, but it is neutral to contestable conceptions of the good and of the person. ${ }^{84} \mathrm{~A}$ second norm is used in conjunction with rational dialogue, namely that of equal respect. This has been examined as a response to pluralism/disagreement already, but, in general terms, it is significant because it encourages people to engage in conversation, and to continue conversation after disagreement results. ${ }^{85}$ So, while the norm of rational dialogue suggests that disagreement results from retreating to neutral ground, it is the respect we hold for others that encourages us to engage in the

\footnotetext{
${ }^{78}$ Ibid, p45.

${ }^{79}$ Ibid, pp45-46.

${ }^{80}$ Ibid, p50.

${ }^{81}$ Ibid, p52.

${ }^{82}$ Ibid, p53.

${ }^{83}$ Ibid.

${ }^{84}$ Ibid, pp54-55.

${ }^{85}$ Ibid, p59.
} 
dialogue. These two values together, Larmore asserts, justify political neutrality and make cooperation possible between people who reasonably disagree.

The resulting political neutrality suggests a modus vivendi liberalism that keeps contested conceptions of the good away from political discussion. ${ }^{86}$ However, as Larmore notes, it may not always be possible to reach an acceptable agreement by this rational dialogue. In that situation it is possible to look to other liberal principles, such as to basic liberties or distributive principles. ${ }^{87}$ This should be carried out in a neutral way, which can be achieved by minimising the loosening of neutrality, by admitting beliefs that are "least central to anyone's idea of the good life" or that "the least number of people do not hold". ${ }^{88}$

The result is a political liberalism structured in terms of a neutral justification of political neutrality, and supported by the norms of rational dialogue and equal respect. This theory is woven into Larmore's three neglected moral complexities, of moral judgment, moral divisions and moral heterogeneity. Each of these complexities has some plausible standing. It seems reasonable to entertain the ideas that abstract moral principles conflict in a fundamental way; that it is possible to split the political, or the socio-moral, from the personally moral, since different general considerations apply; and, that moral judgment can reach a moral result in a way that is not available to theory. Each of these is plausible, but argued only briefly by Larmore. These are fundamental ideas about obviously complex areas, and require greater discussion than Larmore offers.

\subsection{Neutral political neutrality}

Although foundational, these complexities do not feature directly in the theory's substance, and, so, it is possible to examine the latter while remaining open as to whether and how Larmore's arguments of the former succeed. The general structure is one of political neutrality justified by the norms of rational dialogue and equal

\footnotetext{
${ }^{86}$ Ibid, p74.

${ }^{87}$ Ibid, pp67-68.

${ }^{88}$ Ibid, p68.
} 
respect, or, in other words, an ethic of entering into conversations, and of retreating to neutral ground, in the prescribed way when disagreement arises. Political neutrality is supposed to be a direct result of this ethic, but this turns out to be less than straightforward. Firstly, the idea of 'neutral ground' offers a novel conception of neutrality, which implies not the absence of conceptions of the good life, but the absence of conflicting conceptions of the good life. This represents an effective link between dialogue and neutrality, but means that the resulting political neutrality must also be limited to excluding contested conceptions of the good.

The argument, then, is that a commitment to entering into dialogue and to retreating to neutral ground when disagreement arises, in the hope of resolving or bypassing the dispute, means that the state should promote any contested conception of the good in the political domain. There is something disconcerting about this fit between the two norms and the principle of political neutrality. It may be that the norms seem to represent a simple personal ethic about engaging and behaving in dialogue, yet they are being used to justify an eminent political principle. The ethic seems to refer to personal as much as political action, and, so, its application to the political system is a stretch, especially as part of a theory that promotes different moral considerations for different domains.

Even if it were accepted as a universal political ethic, there is a sense in which the two norms are not sufficiently powerful to justify political neutrality. This may relate to the different senses of neutrality used. Rational dialogue implies the retreat to neutral ground, which is then a point from which conversation continues; political neutrality concerns the non-promotion of conflicting conceptions of the good. The link between the two of these is tenuous: using common ground as a neutral mid-point that assists in effective dialogue does not imply that states should not promote particular contested conceptions of the good. While the two may both exclude contested conceptions of the good, they do so for quite different reasons, and the attempt to draw political neutrality from this dialogical neutrality seems to rest on two ultimately disparate ideas.

This result is independent of two other problems Larmore's justification of political neutrality faces. The first is the status of the two related norms. Larmore correctly 
recognises these as being morally substantive and, so, must themselves be justified. He seems to believe them to be universally acceptable. ${ }^{89}$ This in itself is questionable, especially when the norm of rational dialogue is fleshed out so as to include the retreat to common ground in order to attempt to achieve an accord. Even if they are both accepted as universal, this may not be enough for Larmore's purposes. These norms must also be sufficiently important to justify such a politically fundamental principle. If this level is not reached then it would be possible for other norms to justify some conflicting principle, which would undermine Larmore's neutrality.

The second problem Larmore faces is in relying so completely on the distinction between the right and the good. This is implicit in the separation between the enabling norms and the principle of neutrality (the right), and the conceptions that are excluded both from rational dialogue and from justifications of state action (the good). As with earlier liberal theories, this distinction is tempting as the basis for a liberal metasystem that is structured according to the right, and that describes a political system that is open as to the good. However, as with these systems, this distinction is difficult to draw and apply within a moral system.

This is manifested most obviously in Larmore's theory in relation to the neutrality of rational dialogue, which prescribes a stepping back from conflicting beliefs to a place of neutral ground. This is supposed to be neutral with regard to conceptions of the good life only, and not morally. ${ }^{90}$ Yet, rational disagreement can occur in relation to the right as well. The interlocutors might then revert back to agreed matters of the right, but this would mean that the neutral ground would be neutral as to conflicts of the right as well as the good, which would conflict with the idea of political neutrality that it is supposed to justify. Public reason theories must allow for moral disagreement as well as disagreement about the good, and, for this reason, alone basing a theory on the distinction between the two is perilous. Rawls' shift to the broader conception of comprehensive doctrines avoids this problem neatly, but this is not available to Larmore since his theory is based on the neutrality between conceptions of the good.

\footnotetext{
${ }^{89}$ See ibid, p53; c.f. ibid, p97.

${ }^{90}$ Ibid, pp54-55.
} 


\subsection{Larmore's public reason}

Setting aside matters of justification, Larmore's political liberalism is interesting in its focus on the two norms of rational dialogue and equal respect, as opposed to Rawls' liberal legitimacy. It has been criticised, however, on the ground that it may not achieve any accord. ${ }^{91}$

Agreement in Larmore's public reason depends on a mutual cooperative commitment to the two norms, and on the common ground from which political reason ultimately must work. Although common ground in a conversation between two people seems an acceptable basis for partial agreement and further discussion, a political conversation must involve all who commit to cooperation. Given that the environment in which this conversation goes on is characterised by disagreement, that a useful common ground could exist seems unlikely. Certainly, the likelihood of arriving at a useful common ground is more so than with Rawls, whose overlapping consensus is based on a common social context and stricter entry requirements. Larmore does aim only at reaching a modus vivendi, but some level of political agreement would still be required for this to function as a liberal political system. This difficulty, Jonathan Seglow argues, means that the two norms must take a larger role in justifying a political action. ${ }^{92}$ In other words, substantive political content must come from somewhere, and, if not from common ground, then from the other feature common to all participants: commitment to the two norms.

Seglow then identifies respect as the possible source of this material, by reason that it requires us to

justify our position to others on terms that would be unreasonable for them not to accept, where the reasonableness standard... is a universal standard of all rational agents. This universal standard must be what persons mutually respect, not their concrete ends and values which may diverge, but their general capacity to adopt ends and values in the first place. ${ }^{93}$

\footnotetext{
${ }^{91}$ E.g. J S Fishkin, Book Review: Patterns of Moral Complexity by Charles Larmore, (1989) 17(1) Political Theory 153, p155.

92 J Seglow, 'Neutrality and Equal Respect: On Charles Larmore's Theory of Political Liberalism'

(2003) 37 The Journal of Value Inquiry 83, p89.

${ }^{93}$ Ibid, p90.
} 
In order to derive substantive content, respect must be conceived to require people to justify themselves to others in reasonable ways. The standard by which this is carried out respects the capacities of others to adopt ends and values, or, in other words, appreciates their autonomous selves. In this way, Seglow argues, Larmore's public reason can succeed only by incorporating the extra value of autonomy into the account of respect. ${ }^{94}$ The general point here is that there would be no source of agreement in Larmore's political conversation without incorporating other moral conceptions. It is true that Larmore's equal respect contains a hint of autonomy already, in that it is the capacity of others to form respectable beliefs that is respected.

Larmore's response to this is that neutrality can be relaxed so as to reach agreement, by cutting out the least central or most unpopular beliefs. James Fishkin argues that this is most unsuitable:

\begin{abstract}
Either of these approaches is likely to yield policies that transform the liberal state beyond recognition. On 1, suppose that anti-pornography forces see it as absolutely central to their idea of the good that this evil "must" be abolished. On the other hand, few consumers of pornography will view such products as central to their idea of the good, whatever else they might wish to say in favour of the liberty involved. Hence, on criterion 1 , there might well be a case for stamping out pornography, for criterion 1 gives special weight to the centrality of a moral conviction in one's idea of the good. Similarly, criterion 2 might be employed to establish theism or even Christianity, as the number of atheists or even the number of nonChristians is relatively small in many putative liberal states. Criterion 2 would seem to give great power to "moral majorities" who wish to stamp out dissenting or heretical views so long as they are held by only a small number. ${ }^{95}$
\end{abstract}

This passage ignores the fact that Larmore states that this process should be undertaken neutrally, so that other, less controversial moves would be made ahead of those suggested above. In addition, Fishkin ignores the fact that neutrality should be loosened not just to allow any political move, but in favour of other liberal principles.

Despite both of these possible responses, Larmore's argument is poor. It admits of the practical shortcoming of the central principle of his theory, and adjusts to this by giving effect to liberal factors that are not justified or even mentioned anywhere else in his theory. Even if the position is not as dire as is painted by Fishkin, this argument betrays a deep flaw in Larmore's public reason, and suggests that disagreement must be positively overcome by a theory of political liberalism.

\footnotetext{
${ }^{94}$ Ibid, p91

${ }^{95}$ Fishkin, op cit, above note 91, p156.
} 
Overall, Patterns is interesting in contrast with Political Liberalism, in that it is avowedly philosophical. It is ambitions in attempting to accommodate the complexities and contestability of morality by advocating a separate political domain based on a simplified model of the common and the universal. This project is hampered, however, by Larmore's adherence to a conception of liberal neutrality that does not fit well with the approach of political liberalism. It also suffers from being too slight in argument and justification, in regard to both its philosophical underpinnings and its substance. An excellent example of the latter is lack of discussion as to the distinction between the political and the personal, a point essential to any successful expression of political liberalism. Larmore's approach to public reason is interesting in that it aims only at the accommodation of a modus vivendi, but the lack of common ground or belief cannot support even this, and, so, Patterns' attempt fails to build any political substance.

\section{Liberal public reason}

\subsection{Habermas and discourse ethics}

The approach of political liberalism is sufficiently complete and well explored so that it is capable of standing by itself as a contemporary liberal approach; but, it can also be viewed as part of a broader trend to use public reason to answer political and moral questions. Because of the nature of public reason, this approach often focuses more on democracy than liberalism. This is reflected in the work of philosopher Jürgen Habermas, whose work represents the philosophical underpinnings of public reason.

Habermas argues that objective and universal approaches to social theory are possible by focussing not on one's personal sense of rationality, but, rather, on the act of social and moral discourse between people. This communicative action can, he argues, provide a foundation for social analysis that transcends the particular society involved: 
[T] he burden of proof on moral theorists who hope to ground a conception of justice in something more universal than the considered convictions of our political culture is enormous. Because Habermas wants to do just that, the links he forges to action theory are crucial; they are meant to show that our basic moral intuitions are rooted in something deeper and more universal than particularities of our tradition. The task of moral theory, in his view, is reflectively to articulate, refine, and elaborate - that is to "reconstruct" - the intuitive grasp of the normative presuppositions of social interaction that belongs to the repertoire of competent social actors in any society. ${ }^{96}$

For Habermas, the answers to moral questions can be found in a discourse theory of morality, which seeks to achieve universality through the interplay of belief, reasoning and judgment that characterises moral discussion. In the political sphere, this means focussing on the procedure by which public reason is used, and the practical requirements of its institutionalisation. ${ }^{97}$ In this way political morality will be given substance by this process of public reason, as the outcome of the institutional organisation implied by Habermas' discourse ethics.

This explanation of Habermas' work is the barest possible, but it is sufficient to emphasise the fundamental differences between him and other theorists who employ public reason. A Theory of Justice, for example, provides an interesting contrast with Habermas in that it employs intuitionism as part of the reflective equilibrium in order to reach universal moral conclusions. The difference is that this result is reached through the operation of individual rationality and belief, rather than through the universalising effect of discourse. Even the original position, which is framed in terms of an interaction between people, does not favour discourse over personal reasoning, since Rawls' conception of representation means that it could function as well with one person as with any other number. What is missing is a conception of a process by which discourse transforms moral disagreement into some form of agreement in a procedurally acceptable way.

Political Liberalism does involve using public reason in this way, although this process is directed and restricted by other aspects of Rawls' theory. The goal of this reason is not to achieve moral truth or universality, as with Habermas, but merely to reach a reasonable, mutually acceptable agreement. Many of the political theories that

\footnotetext{
96 T McCarthy, 'Kantian Constructivism and Reconstructivism: Rawls and Habermas in Dialogue' (1994) 105 Ethics 44, p47.

${ }^{97}$ Ibid, p49.
} 
employ public reason follow this lead in having aims more humble than the achievement of universal moral truth.

This discussion of Habermas' public reason is not sufficient to allow us to judge the success of his enterprise, but it can at least point to the challenges that this general account of ethical discourse must face. There seem to be two, closely linked, fundamental challenges: the first is to show how and why a conversation between people can result in a morally valuable outcome in a way that personal reflection and reasoning cannot; and the second is to explain and incorporate within this account how disagreement survives this discourse, and how a valid and singular moral result can be found despite this inevitably continuing difference.

Taken generally, these two issues apply in some form to any theory of public reason: the latter is directly relevant to theories such as political liberalism; the former is relevant also, although different theories attribute to public reason different capacities and virtues. In Rawls' case, for example, it seems simply the means by which an agreed political conception can be reached, and coercive state action justified. It does not have the special power to move from difference to truth, and so it is less of a task to Rawls to explain how it works. For Larmore, public reason is more dynamic, and he places a greater emphasis on the opportunity discourse offers to work out our differences, by first reverting to common ground. From the discussion of Habermas' approach it seems that public reason offers more than either of these theorists adopt: it could be the basis for and account of social and moral transformation towards a particular moral goal. ${ }^{98}$ But, irrespective of how public reason is used, these two challenges must be addressed.

\subsection{Deliberative democracy}

Because public reason expresses the will of the members of a society generally; it has a ready affinity with ideas of democracy. Democratic theories that focus on public reason look to the deliberation of citizens about social questions as being central to

\footnotetext{
${ }^{98}$ C.f. Seglow, op cit, above note 92, p94.
} 
guiding state action. An important exposition of deliberative democracy is contained in Democracy and Disagreement (1996), by Amy Gutmann and Dennis Thompson.

Gutmann and Thompson describe deliberative democracy simply as meaning that, "when citizens or their representatives disagree morally, they should continue to reason together to reach mutually acceptable decisions". ${ }^{99}$ They place persistent moral disagreement at the centre of their account of democracy, and suggest that this disagreement may well reside in the human condition itself. ${ }^{100}$ At the very least, it is a fact of our existence that there are moral conflicts between people and between values that cannot be collectively resolved. ${ }^{101}$ Democracy represents an appropriate response to this problem, as it "accords equal respect to each citizen, and is therefore morally justifiable from the perspective of each citizen". ${ }^{102}$ Deliberation has a number of virtues as a potential aspect of this democracy: it involves considering people's claims on their merits, and, so, makes the outcome more legitimate; it encourages citizens to take a broader, more moral view of issues; it helps citizens understand the nature of a moral conflict; and finally, by involving many people it makes reaching a justifiable outcome more likely. ${ }^{103}$

These virtues are an interesting part of Gutmann and Thomson's theory of deliberative democracy, as it endues public reason with special moral power over and above that of individual reason. The first three of these virtues characterise public deliberation as being genuinely inclusive and explicitly moral in nature. The latter encourages citizens to engage in deliberation, and to view it from a moral perspective. This can only assist in reducing the role of self-interest in politics, and in promoting a commitment to act in good faith to reach meaningful agreement. The possibility of an agreement is improved through ensuring that, even if people's interests are not met, people are aware that the process includes and shows respect to everyone equally.

\footnotetext{
${ }^{99}$ A Gutmann \& D Thompson, Democracy and Disagreement (1996), p1.

${ }^{100}$ Ibid, p21.

101 Ibid, p25.

102 Ibid, p26.

${ }^{103}$ Ibid, pp41-43.
} 
The fourth virtue responds to the truth of an imperfect human understanding of moral issues. This incompleteness, Gutmann and Thompson argue, is most likely to be overcome by deliberation.

Through the give-and-take of argument, citizens and their accountable representatives can learn from one another, come to recognize their individual and collective mistakes, and develop new views and policies that are more widely justifiable.... When [people] deliberate, they move beyond the conventional patterns of group politics that characterize the standard conceptions of interest group bargaining. ${ }^{104}$

Deliberation allows new and better moral ideas to be developed and endorsed, and steers democracy away from interest and power politics. Overall, Gutmann and Thompson present a deliberative view of public reason that aims at agreement by encouraging politics to be more moral and less personal. This in itself is interesting, in that they allow public reason to carry some of the weight that Rawls assigns to the restrictions of reasonableness: the willingness to treat the political domain as separate from the personal, and to be fair and cooperative when acting in it. This approach is superior in that the moral character of the political comes out of public reason itself, rather than being enforced as an external restriction. This only represents a potentiality of public reason, however, and could never be as stringent as Rawls' approach, which is defined by the restrictive condition of reasonableness.

In addition, Gutmann and Thompson seem to attribute to deliberation a benefit beyond merely encouraging moral political behaviour, and one that is most obvious in relation to the fourth virtue. The idea is that public reason actually improves moral content; it corrects personal mistakes and allows moral development that is more justifiable. This suggests that public reason may have a greater role than just relocating moral uncertainty in a substantive political process, and may actually act to overcome some of this uncertainty, by virtue of adding to our understanding. This is expressed rather modestly by Gutmann and Thompson as correcting our mistakes, and the nature of these mistakes is not explained; but this seems to indicate more than just mistakes of fact upon which moral beliefs are based. It suggests that, contained within public deliberation, are the seeds for genuine moral development: not that moral disagreement will completely disappear, but that at least there is the real prospect of improvement.

\footnotetext{
${ }^{104}$ Ibid, p43.
} 
It could be argued against this point that such improvement is not limited to deliberation. Personal moral reasoning may be undertaken by one person, but it is never carried out in a vacuum. If common moral understanding can be improved through deliberation, then an individual's moral understanding can also be improved by personal experience, research and contemplation. The essential difference between these two ideas is that, with the latter, the process of reasoning, no matter how much others might contribute to it, is ultimately individual; it eschews equal concern for the views of each person in favour of individual judgment. In this way, Gutmann and Thomson's point stands: the mistakes made by that individual might be corrected by the genuine involvement in a moral conversation. Nevertheless, a mistake made by an individual, despite their experiences and research, might easily transfer to their participation in a public deliberation on the same topic. Although Gutmann and Thompson's argument - that deliberative democracy offers a positive response to moral uncertainty - seems valid, it is unfortunate that they do not develop this idea, and, in particular, do not compare the natures of deliberative and personal reason.

The remainder of Democracy and Disagreement performs two tasks. The first is to explore the procedural principles by which public deliberation is best carried out. Gutmann and Thompson identify three: reciprocity, publicity and accountability. Reciprocity is the most important of these, since it shapes the meanings of the latter two. ${ }^{105}$ This principle expresses "a sense of mutuality" that people should bring to deliberation, which encompasses the commitment to supporting their arguments with "reasons or principles that can be shared by fellow citizens who are similarly motivated". ${ }^{106}$ It also incorporates the condition that people should be morally accommodating, which means that they should, in their deliberation, exhibit both integrity and open-mindedness. ${ }^{107}$ The other two principles require that political justification be both public, and addressed to those who would be affected by a decision. $^{108}$

\footnotetext{
105 Ibid, p52.

${ }^{106}$ Ibid, pp52 55.

${ }^{107}$ Ibid, pp79-83.

${ }^{108}$ Ibid, pp95, 128.
} 
Such conditions are requirements of any system of public reason; the concern is as to whether their substance is not itself morally contestable. Here, reciprocity is also said to include the condition that empirical statements be consistent with reliable methods of inquiry. ${ }^{109}$ This would exclude reference to any religious texts that defy a literal understanding of their contents, for example. In this way, Gutmann and Thompson remove from deliberation any religious understanding. The moral prescriptions set out in religious texts are still given appropriate respect, but their doctrinal justification cannot be part of the moral discourse. This seems a reasonable approach in a plural society, but would not encourage those whose moral systems are essentially religious to agree to the deliberative system. What is required here is the kind of separation between political and personal as drives political liberalism, so that those whose moral reasoning is prevented from being part of the political reasoning accept this, given their commitment to cooperation and public agreement.

These procedural conditions on deliberative democracy, Gutmann and Thompson argue, do not sufficiently constrain democratic discretion. A set of constitutional principles are needed in order to set particular standards on citizens and their representatives. ${ }^{110}$ These serve to "inform and constrain the content of what democratic deliberators can legitimately legislate". ${ }^{111}$ Here Gutmann and Thompson arrive at three principles: basic liberty, basic opportunity and fair opportunity. Basic liberty concerns the limits on personal voluntary action; basic opportunity, the responsibility to provide for people's basic needs; and fair opportunity, the means by which citizens can strive to gain the resources to live their lives as they choose. ${ }^{112}$ Each of these principles uses particular examples from recent US political history to illuminate the central issues at stake, and to set limits on any subsequent deliberation, and all are explored keenly by Gutmann and Thompson.

Democracy and Disagreement could easily be taken to be a theory of liberalism in light of these apparently liberal principles, and the central requirement that coercive state action is justified to those whom it affects. It is inevitable that liberalism based on public reason is closely allied to a form of democracy. Democracy and

\footnotetext{
${ }^{109}$ Ibid, p56.

${ }^{110}$ Ibid, p199.

${ }^{111}$ Ibid, p200.

${ }^{112}$ Ibid, see chapters 7-9.
} 
Disagreement bridges the divide between the two from the other direction, and, so, might be labelled a theory of liberal democracy. Yet, it is this liberal content that proves to be the most problematic and most interesting part of the theory. The main problem is that the theory champions public deliberation as the best response to persistent moral disagreement. However, these principles amount to substantive moral constraints; and they are a result not of public deliberation, but of the reasoning of Gutmann and Thomson. Daniel Weinstock persuasively identifies two aspects of this inconsistency:

First, given that the authors are concerned to avoid the kind of foundationalist arguments sometimes relied upon by liberals, how do they justify their own first principles? Democracy itself cannot be invoked, since the principles are brought in to constrain democratic decisionmaking procedures. And moral considerations also must be avoided, since they might be disowned by some citizens.... Second, should a book that purports to set out how decisions about public policy should be reached by citizens and their representatives... spend as much time as this one does coming to determinate conclusions about how the just society ought to deal with such questions as health care, affirmative action, environmental protection and the like ? $^{113}$

Weinstock argues that the inclusion of substantive liberal principles is both unjustified within the structure of the theory; and, is contrary to the process of deliberation because it employs "the nondeliberative tools of the professional philosopher". ${ }^{114}$

These arguments are not entirely fair. The first one supposes that Gutmann and Thompson are undertaking a process of substantive moral reasoning that is entirely at odds with the aim of public deliberation, but a closer look at the content of these principles undermines such a view. The discussion of liberty and opportunity by Gutmann and Thompson is not a direct alternative of democratic deliberation. Rather the conditions of deliberation and principles of liberty and opportunity "inform the content of deliberation, all appropriately ordered and interpreted over time by the people bound by these principles". 115

This means that the principles of liberty and opportunity do not represent substantive moral ideas such as could be the outcome of deliberation, but, rather, are partial principles that guide the process of deliberation. This is illustrated by their analysis of

\footnotetext{
${ }^{113}$ D M Weinstock, Book review: Democracy and Disagreement by Amy Gutmann \& Dennis Thompson (1997) 91(3) The American Political Science Review 724, p724.

114 Ibid.

115 Gutmann \& Thompson, op cit, above note 99, p201.
} 
these principles. In referring to actual political examples, Gutmann and Thompson do not simply declare a substantive result, but rather examine the issue from a perspective of deliberation: they look to explain and judge them as actual deliberative examples; they are guided by what could be called a liberal middle ground, which reflects both the liberal skew of deliberation itself, and the desire to reach results that are justifiably acceptable to all citizens; and, finally, they analyse the issues in terms of the deliberative steps that should be met, and, so, leave open space within which people can apply their personal moral beliefs in order to reach a decision.

This approach is interesting in that it seems to anticipate a public reason that is not merely a conversation between people with different moral values, but that is closely guided by various necessary analytical processes. On this account, Weinstock's criticisms retain some power. It is difficult to see why this deliberative democracy would be accepted by those who disagree with its liberal content, and, so, it might be rejected by the devout religious believer, the libertarian or the egalitarian. Gutmann and Thompson allow that the content of the liberal principles responds to deliberative outcomes and, so, can shift over time. ${ }^{116}$ However, this cannot quite overcome the unchanging liberal perspective and liberal terms of reference by which the deliberation is undertaken. In relation to Weinstock's second criticism, it is true that there is a discord between this substantive liberal content and the use of deliberation, despite the deliberative eye that Gutmann and Thompson cast on the liberal issues. For their approach to be acceptable they would need to place both deliberation and the non-deliberative tools they employ into a broader model of politico-moral decisionmaking.

As it stands, Democracy and Disagreement is an attempt to embed moral disagreement into a theory of democracy. As such, it has the same structure as a liberal meta-system: persistent moral disagreement is made the subject of a process of public deliberation, which itself must be explained and justified. The overall justificatory structure involves two levels of agreement: firstly, about a particular issue that is the subject of a public deliberation; and, secondly, about the acceptability of the system of deliberation itself. Moral disagreement, however, cannot be restricted

\footnotetext{
${ }^{116}$ Ibid, pp200-201.
} 
to the first of these. Instead, the theory itself, particularly its liberal content, is the valid subject of such disagreement. Yet without the liberal content, it would be unclear that substantive agreement could ever be reached. This indicates a fundamental dilemma in public reason theory: public reason needs to be informed or constrained in such a way that some manner of agreement can result, and, yet, these constraints must themselves be acceptable to people with diverse beliefs. ${ }^{117}$ This is a political version of the general problem identified with pluralism, namely of finding a unity out of difference.

\title{
4.3 Justificatory liberalism
}

Gerald Gaus makes a similar point about deliberative democracy. His argument stems from the nature of political disagreement: that for any doctrine there are those who believe it not to be merely wrong, but unreasonably so.

\begin{abstract}
The problem is that we do not simply live in a society with plural understandings of the good life, but with diverse and conflicting ideologies that insist their competitors are deeply misguided. None of this is to say that political life in an ideologically fractured society is impossible. It does, however, strain beyond plausibility the claim that politics ought - even ideally - to aim at actual consensus. ${ }^{118}$
\end{abstract}

This means that deliberative democracy is mistaken in that it aims for an agreement that it can never even come close to achieving. In relation to Democracy and Disagreement, this would seem to translate to disagreement about the liberal principles, as liberalism would represent an ideology that opposes the honestly held ideologies of others.

Gaus levels a similar criticism against Rawls' political liberalism. He suggests that the political domain is itself characterised by reasonable pluralism in a way that cannot be adequately dealt with by a theory aiming at near-universal acceptance. ${ }^{119}$ In making this assertion, however, he does suggest that liberalism has special moral content.

\footnotetext{
${ }^{117}$ See Weinstock, op cit, above note 113 , p724.

${ }^{118}$ G F Gaus, Contemporary Theories of Liberalism: Public Reason as a Post-Enlightenment Project (2003), p140.

${ }^{119}$ G F Gaus, 'Reasonable Pluralism and the Domain of the Political: How the Weaknesses of John Rawls's Political Liberalism Can be Overcome by a Justificatory Liberalism’ (1999) 42 Inquiry 259, p281.
} 
Rawls, I believe, is correct in that there is conclusive rational consensus on the basic concept of a liberal regime, and the broad outlines of liberal principles. But consensus only characterizes liberal principles at the most abstract level. As in many other complex matters, the use of human reason under free institutions leads us to disagree. The political cannot be insulated from this, nor can the realm of public reason be convincingly constricted so as to not be effected by it ... Because the pluralism of the political manifests our different interpretations of the demands of liberal justice, the political is constituted by a plurality of articulations of public reason, of citizens seeking to construct arguments to convince each other about the best interpretation of the demands of justice. ${ }^{120}$

This envisages an abstract agreement with liberalism that transcends the ideological fracturing of society posited above. It may be political in nature, but it is real and common between us all. This does not, however, engender actual political agreement, as our individual reason leads to a plurality of conflicting conceptions. Presumably this operates in the same space as the conflicting ideologies, and it would make sense for the latter to be simply conflicting conceptions of the former.

Gaus states that the resulting political disagreement is unable to be overcome by public reason. He seems to imply that any system of public reason must itself result in a pluralism of outcomes, or must constrain this reason so that the system itself no longer reflects the full breadth of the pluralism, as does that of Gutmann and Thompson. This suggests that the common liberal core is so abstract that it cannot, by itself, imply a particular substantive liberal ethic and system of justice, and, so, cannot be reconstructed in the Habermasian sense. The idea of a liberal core that is then given political expression by a process of public reason is an attractive one in that it allows persistent moral disagreement, yet includes liberal content at the core of a system of public reason. This content is not contestable on the same level as a political issues, as it is, by nature, politically incorporeal, and, yet, is genuinely common and moral. Such an approach, however, depends on the difficult feat of identifying and justifying this core as described.

Gaus' approach to this is set out in his book Justificatory Liberalism (1996). It amounts to an explicitly liberal approach to public reason, and identifies public justification, both, as the proper form of public reason, and, as the central liberal concern. This requires that every political decision be justifiable to those whom it affects. Gaus admits that universal acceptance of justified liberal principles, or any

${ }^{120}$ Ibid, pp281-282. 
other moral principles, is not realistic. Instead, what is required is a normative theory of justification that allows principles to be said to be publicly justified despite being contested by many. ${ }^{121}$ In order to provide such a theory, Gaus argues that a detailed understanding of the nature of moral justification is required, or, in other words, a model of moral epistemology. This model will direct liberals as to what public justification actually requires. Gaus then uses this model in order to justify his conception of a universal liberal core. The structure of Gaus' liberal justification, then, is that his liberal conception is publicly justifiable according to a valid epistemological system. He breaks down this process into three steps: developing an understanding of personal moral beliefs, and, so, of personal justification; applying this to a public reasoning process; and, then, justifying political principles according to this public justification.

Gaus' account of personal justification is complex, and borrows from a number of epistemological ideas, as well as recent work in psychology. He states that justified personal beliefs must be causally supported by reasons that are both accepted and good. ${ }^{122}$ These conditions imply that there is an actual and valid inferential link between the justified belief and the reasons for it. Gaus refines this position by applying a conception of open justification whereby a justified belief system is "stable in the face of acute and sustained criticism by others and of new information". ${ }^{123}$ The answer to this depends on their particular set of beliefs, which leads Gaus to admit that reasons are relative to the belief systems of their holders, but to suggest that this relativism does not extend to truth itself, just the reasons we use our justify our beliefs. ${ }^{124}$ This does not, he argues, reduce personal justification to an unworkable relativism. Instead justificatory reasoning features widespread shared beliefs and inferential norms that allow meaningful interaction between people. ${ }^{125}$ In addition, the mere acceptance of a belief does not imply that the reason that supports it is valid, nor does it exclude the possibility that the person is in error; in fact, psychology boasts many examples of common inferential errors. ${ }^{126}$

\footnotetext{
${ }^{121}$ G F Gaus, Justificatory Liberalism: An Essay on Epistemology and Political Theory (1996), p4.

${ }^{122}$ Ibid, pp17-19.

${ }^{123}$ Ibid, p31.

${ }^{124}$ Ibid, p39.

${ }^{125}$ Ibid, p49.

${ }^{126}$ Ibid, pp54-58.
} 
The justification of a belief by one person to another does not, Gaus argues, require a person actually change their mind and accept the justified belief. That person may withhold their agreement for reason of "obstinacy, selfishness, laziness, perversity or confusion". ${ }^{127}$ What is instead required is that the belief is justifiable given the other person's existing set of beliefs and reasons. ${ }^{128}$ This concept both accords with the personal moral epistemology, and retains the moral content of a valid and recognisable conception of public reason. But, given that justification must be made in relation to the beliefs of the other party, the standard of social agreement would seem extremely high. Gaus recognises that attempts at public justification can have three outcomes. The first is that the justification is defeated by the receiving party, meaning that it is shown not to be justifiable relative to their existing beliefs and reasons. ${ }^{129}$ The second is that it is victorious, which requires that the receiver fails to point to any inconsistency between the justification and an existing, valid belief, but, also, that two conditions are met: that others have had the opportunity to present counter-arguments, and that the claim of victory meets a high evidential standard, similar to that of going beyond reasonable doubt. ${ }^{130}$ In this way, the standard of justificatory victory is more stringent than that of defeat, even in relation to two parties.

Gaus also recognises a third possibility, where justifications are undefeated but not victorious. Typically, this outcome occurs because the arguments supporting the belief are inconclusive in that the matter is of such a complex nature that the standard of proof is difficult to meet. ${ }^{131}$ In such cases, a further decision-making process is required, which Gaus identifies as the adjudication of an arbitrator. He sees this democratic institution as resembling the umpire in a game:

The umpire's aim is to produce practical determinations that best track what the rules require. Yet players need only conceive of her as a practical authority.... Indeed it follows that players are committed to accepting the umpire's practical authority even when they believe her decision is wrong. ${ }^{132}$

\footnotetext{
${ }^{127}$ Ibid, p131.

${ }^{128}$ Ibid, p138.

${ }^{129}$ Ibid, p146.

${ }^{130}$ Ibid, p150.

${ }^{131}$ Ibid, p152.

132 Ibid, p189.
} 
In this way, a democratic umpire uses their judgment, as informed by various limits and standards set by justificatory liberalism, to decide in cases of inconclusive justifications. The outcomes of this can be validly accepted by all citizens involved, without any committing to altering their own beliefs to match. ${ }^{133}$

This expression of adjudicative democracy is an important aspect of Justificatory Liberalism. Its relationship to the moral epistemology is interesting, in that it accords with Gaus' view of beliefs as potentially flawed, and steers away from the goal of public consensus. The other interesting part, especially in comparison with Gutmann and Thompson's deliberative democracy, is the importing of substantive liberal content into the theory. This is achieved in two different ways. Firstly, Gaus identifies those liberal principles that are so intimately bound up with public justification that they are justified merely as a consequence of the public justification itself.

The corollary of requiring the public justification of socially significant decisions is that those decisions that are not justified should not be imposed on people (excepting, of course, through the operation of the adjudicative process). This implies a liberal principle of toleration, since public action that affects others cannot be taken without first going through the process of justifying that action to them. ${ }^{134}$ Toleration, in this form, "is a minimal but fundamental requirement that we not impose upon others without justification". ${ }^{135}$ In addition, free speech is fundamental to public justification, and, in particular, to the publicity requirement of victorious justification. ${ }^{136}$ This reflects a commitment to public reason, and the free and genuine action of those who participate in it. It is not surprising that these liberal conditions of public justification are required, given that justification as a standard of legitimacy is a liberal idea. The values of toleration and free speech, then, are as valid as the process of public justification itself. The only concern is that they are given no more extensive meaning than is implied by public justification, so that, for example, toleration means nothing more than the absence of public action undertaken without an appropriate justificatory basis.

\footnotetext{
133 Ibid.

${ }^{134}$ Ibid, p162.

${ }^{135}$ Ibid, p164.

${ }^{136}$ Ibid, p167.
} 
The second way in which Gaus incorporates substantive liberal values relates to his comment above, that there is an abstract rational consensus as to core liberal beliefs. For this reason there is a set of abstract liberal principles that are mandated by the action of public justification, and, in particular, by his mechanism of immunity. If many attempts have been made to publicly justify a particular belief or set of beliefs, and this has failed, then this is good evidence that the attempt is unlikely to ever succeed, and that citizens should rightly be held immune to such attempts. ${ }^{137}$ The example he offers is of the failed attempts to apply religious doctrine on others during the politics of the sixteenth and seventeenth centuries, and of the practical US expression of this failure, the prohibition on Congress abridging freedom of expression or religion in the first amendment to the US Constitution. ${ }^{138} \mathrm{He}$ also makes something of pluralism here, arguing that the fact that many believe in a plurality of views of the good life means that attempts to justify a single such view will necessarily fail. ${ }^{139} \mathrm{He}$ labels it a defeater doctrine, in that even though it may not be conclusively justified, it will serve to defeat alternative arguments. Gaus carries the idea of immunity into a conception of privacy that prohibits the public justification of beliefs that are based on reasons of a personal moral dislike for an activity, disapproval or offence. ${ }^{140}$ Such reasons would always be defeated by people who did not share that dislike or that sensibility, including the participants themselves.

Gaus goes on to discuss the basic interests which, liberalism would tend to suggest, should be protected. These include, in the words of Locke, "life, liberty, health and indolence of body; and the possessions of outward things, such as money, lands, houses, furniture and the like". ${ }^{141}$ The basic liberties are the subject of hypothetical contractual arguments such that all rational people would accept norms in favour of their protection. Gaus argues that this has particular meaning in a justificatory framework:

Although social contract theorists have often relied on some form of actual consent to justify public enforcement of these norms, it should be manifest that, according to justificatory

\footnotetext{
${ }^{137}$ Ibid, p170.

${ }^{138}$ Ibid, pp168-169.

${ }^{139}$ Ibid, p171.

${ }^{140}$ Ibid, p172.

${ }^{141}$ Ibid.
} 
liberalism, hypothetical contractual arguments are genuinely justificatory. When the hypothetical contractualist argues that all rational individuals would consent to norms protecting these interests, it is being claimed that we all have conclusive reasons to adopt these norms. ${ }^{142}$

This means that hypothetical agreement translates into a conclusive justification, since all have reason to agree with it regardless of their own set of beliefs and values. The nature of the hypothetical agreement about these basic liberal norms is that they are preferable to a non-protectionist state of nature. ${ }^{143}$

This justification of the inclusion of substantive liberal content is both novel and ingenious. It consists of two conceptions: immunity and conclusive justification. The immunity argument is that those justificatory claims that depend on the acceptance of a single religion or way of life, or on particular moral sensibilities, will necessarily be defeated by the beliefs of others. This result depends on the nature of the claims involved: claims based on a contested religion, a contested way of life, or on moral sensibilities that cannot be shared by the subjects of the justification, will not be sufficiently justifiable to them, as such arguments would be contrary to their honestly and rightly held beliefs and reasons. In other words, it is the personal and contingent nature of the reasons behind the justification that means that they will necessarily be defeated in a social context.

This is an elegant philosophical justification for the liberal principles of freedom of religion, the freedom from interference based on moral offence or dissatisfaction, and a pared-back neutrality based on pluralism. These ideas are derived convincingly from public justification. The chief concern about the approach of immunities and defeater doctrines, is that they may apply to a broader group of beliefs than Gaus anticipates. If the only requirement is that a contrary doctrine is openly justified by another person, then the list of immunities should be quite long.

An initial possibility would be to extend Gaus' idea of pluralism as a defeater doctrine from reflecting plural ways of life, to a thoroughgoing value-pluralism. If it is an openly justified belief of some citizen or group of citizens that moral value is by nature plural and incommensurable, then any public justification will be unable to

${ }^{142}$ Ibid, p173.

143 Ibid. 
promote any particular moral value. The scope of such defeater doctrines turns out to be so broad that it is easy to envisage the possibility of conflicting defeater doctrines: value-monism, as an openly justified belief, would act to prevent a belief based on pluralism from ever being publicly justifiable.

This approach seems to suggest an unattractive position whereby any possible justification is defeated by another openly justified belief. This would create a stalemate in public reason, and one that an umpire could not deal with, since this umpire is only supposed to deal with the inconclusive result of the undefeated but not victorious justification. The problem here is that in a diverse society any justification is likely to be defeated in some way by at least one other person, and the liberal freedoms are no exception to this. Gaus' attempt to derive some liberal content from public justification seems to suffer from the lack of liberal restrictions on valid beliefs. There does seem to be a real and substantive link between public justification and restrictions based on beliefs that are individual and necessarily not shared. But, using an effectively unanimous standard of defeat within a diverse population means that this effect would spread much wider than Gaus would anticipate or accept.

The second approach to incorporating liberal principles is through conclusive justification. Gaus argues that the basic liberal core of liberal principles of protection would be chosen by everyone, rather than facing the non-protection of a state of nature. Again, this argument is a clever attempt at drawing substantive liberal content from principles of public justification. There is certainly power to the idea of the rational choice of protection over vulnerability, but this is protection in its most abstract form. Gaus appeals to the common liberal core, through which the exercise of personal reason takes particular form. ${ }^{144}$ The main concern here is whether this abstract ideal of protection is in fact necessarily liberal. Some forms of protection seem fundamental, but the possible conceptions of this protection are diverse, and may encompass ideas that are not recognisably liberal. This turns on the forms of protection that might be rationally conceived by someone in the situation of hypothetical consent. The issue is whether the rationality of such an individual constrains them to a liberal account of protectionism. Gaus may argue that it does, and

${ }^{144}$ See above, note 120 
he may be right, but, even then, the liberal content would have only the most abstract form, and would not act as a substantial constraint on future public justification.

Gaus' arguments from public justification to liberal content are interesting and potentially important, but have not drawn the critical attention they deserve. The attempt to derive a basic, abstract set of liberal rights from a system of public justification is a significant undertaking, especially since Gaus relies on an abstract and universal liberal core of values. Ultimately, however, they face problems that Gaus does not deal with, and which require further explanation or substantial reformulation.

This derivation of substantive liberal content amounts to the most embedded of the three layers of justification that make up Justificatory Liberalism. The next concerns Gaus' conception of public justification. As Mark Evans points out, public reason theorist Fred D'Agostino has allowed that the actual process of justification is itself contestable:

\begin{abstract}
Liberals have rendered it into a variety of conceptions which are not all compatible with each other, and for which no authoritative method of ranking exists. D'Agostino contends that conceptions of public justification diverge on whether they argue (i) for a deep consensus on principles between citizens, or a convergence in practice from diverse viewpoints; (ii) that X is legitimate if it is reasonable to accept it, or that it is not reasonable to reject it; (iii) that $\mathrm{X}$ is legitimate because it advances people's desires or that they believe it to be right; (iv) that X is to be justified to actual agents or their 'ideal', hypothetical representatives; (v) that justification reflects mutual advantages - a compromise of each relevant individual's interests - or judgments of the common good from a shared standpoint; (vi) that $\mathrm{X}$ requires justification before implementation or that justification is obtained if it is reasonable to assume it will be publicly accepted; (vii) that justification requires interaction (argument, bargaining) between the parties involved or no interaction (in which people reach their conclusions separately). ${ }^{145}$
\end{abstract}

This shows substantial diversity among possible liberal conceptions of public justification. Gaus' task, then, is to justify his particular conception as valid. Much of this is achieved by founding this conception on his epistemological account of justification, and this is one of the strengths of Justificatory Liberalism: that public justification is not merely the product of a philosophical or moral account of the requirements of legitimacy, but, rather, reflects an accurate model of the way we validly hold beliefs, and the way that different beliefs are genuinely justifiable to us.

\footnotetext{
${ }^{145}$ M Evans, 'Is public justification central to liberalism?' (1999) 4(1) Journal of Political Ideologies $117, \mathrm{p} 119$.
} 
The main goal of Justificatory Liberalism is to avoid these issues of contestability by offering an epistemologically realistic basis for justification.

This approach leaves Gaus open to objections against particular aspects of his epistemology, as well as for the parts of his account of legitimacy. Both Gaus' justificatory epistemology and his attempts to derive liberal principles from it face significant challenges, but are interesting and potentially valuable moves in liberal theory. Overall, Gaus' approach is a robust attempt to justify aspects of his model of justification.

The final, and most general level of justification that Gaus, and, in fact, all liberal public reason theories must face, is in asserting the central moral importance of public justification to liberalism. One of the first and most significant statements of this nature was made by Jeremy Waldron in his seminal article 'Theoretical Foundations of Liberalism.' His central thesis is that

\footnotetext{
liberals are committed to a conception of freedom and of respect for the capacities and the agency of individual men and women, and that these commitments generate a requirement that all aspects of the social should either be made acceptable or be capable of being made acceptable to every last individual. ${ }^{146}$
}

He goes on to comment that this view will not be recognisable to every liberal, but that it does underpin much of what it means to be liberal, and does reflect one perspective of liberalism. ${ }^{147}$ He characterises that part of liberalism that is represented by the public justification requirement, as including those conceptions that base political legitimacy on the consent of the citizens, and that encompass contract arguments. ${ }^{148}$ These theories, he argues, face several difficulties, including the impossibility of complete agreement, unreasonably withheld consent, and mistaken and unreasonably held beliefs. This leads the liberal to search not for accord, but for an approach to justifying their beliefs to all. ${ }^{149}$

\footnotetext{
146 J Waldron, 'Theoretical Foundations of Liberalism' (1987) 37 The Philosophical Quarterly 127, p128.

147 Ibid.

148 Ibid, p140.

${ }^{149}$ Ibid, p144.
} 
This shift from actual consent to liberal justification, Waldron recognises, faces difficulties. Such a liberal system is sensitive to the irreducible pluralism and diversity in society, and can base political discussion on a common commitment to some ideal of the good life. ${ }^{150}$ But this does not take into account the potential for deep difference that overwhelms even the abstract liberal conceptions of life, and of illiberal views of life. The liberal, Waldron argues, must limit liberalism to those with existing liberal beliefs. ${ }^{151}$ This means that the resulting liberal politics is not neutral as to liberal beliefs.

Waldron's perspective on liberalism is one that is broadly concerned with justifying social policies to all individuals. He does acknowledge, however, the inherent difficulty with justifying this system of justification itself, except to those who are already liberal and already broadly accept the content of such a system. Although he asserts public justification as a central conception of liberalism, he does not view the resulting liberalism as universally valid, but, rather, contingent on existing liberal beliefs.

Gaus also argues in favour of the centrality of public justification to liberalism. He borrows an example from Stanley Benn, where one person, Alan, splits pebbles on a public beach and another, Betty, seeing the act taking place, takes measures to prevent it continuing. Gaus asserts that this example demonstrates the quintessential liberal claim "that there is a basic asymmetry between you acting and another interfering with your actions". ${ }^{152}$ The nature of this asymmetry is that Betty must justify her actions in interfering with Alan's behaviour, yet Alan is under no obligation to justify his actions to her.

Benn's point is not simply that Alan and Betty may see the world this way, but it is almost impossible for them (and us) not to see social relations as being governed by this principle of non-interference.... Betty is setting about thwarting his agency without being able to give him any good reason; she is trying to undermine Alan's ability to decide what he will do. As do we, Alan sees an important asymmetry between himself and others in deciding what he will do: unless good reason can be provided to the contrary he is the one who makes decisions about what he will do. It takes a special case for Betty to interfere. As John Stuart Mill said, '[o]ver himself, over his own body and mind, the individual is sovereign'. ${ }^{153}$

\footnotetext{
${ }^{150}$ Ibid, p145.

${ }^{151}$ Ibid, p146.

${ }^{152}$ Gaus, op cit, above note 118, p207.

153 Ibid.
} 
Gaus argues that in this situation Alan should, by default, have the right to act. Betty can only overcome that right if she has good reason. This pattern of personal choice over one's personal actions in the absence of contrary public justification is, Gaus argues, at the very heart of the liberal ideal. Allowing interference without the possibility of this justification would undermine individual agency and arouse legitimate feelings of resentment or indignation.

There is a basic problem with this claim. It is not that the pattern of interference is misapplied, but, rather, how this interference is recognised, and, in particular, how it is distinguished from personal, protected action. In the above example Alan desires to split pebbles on a public beach, and Betty desires that he should not. It seems obvious that Alan is acting within a personal domain, which should draw the protection of Gaus' liberal presumption, and it is equally clear that Betty is attempting to interfere in the actions of another. A closer look suggests that this situation may not be so obvious: Alan wants to act in a way that seems to negatively affect Betty, since she sees some reason to prevent it; and Betty wants to act in a way that negatively affects Alan, since he wants to continue.

The basis for Gaus' analysis of this example is that Alan's action is protected. The reasoning behind this seems to depend on the fact that it is an inconsequential act that is an expression of Alan's choice and his agency, and is a physical act of his own person. ${ }^{154}$ This does not seem as necessarily inconsequential as Gaus suggests: Betty has been driven for some unspecified reason to act to physically prevent it continuing. It may be that she is morally indignant that Alan should undertake such a pastime this is a strange justification, but hers is a strange action. Whatever the reason it is possible that the continuation of the activity would cause Betty some form of harm.

If it is accepted that Alan's action is not necessarily inconsequential, then it is difficult to see the logical distinction between his act and Betty's. The physical action taken to prevent Alan from continuing is an expression of her choice and agency in the same way that Alan's act is an expression of his. It is certainly a personal physical act, over which she should be sovereign, and, so, it seems that there is no good reason to deny

${ }^{154}$ Ibid. 
her the liberal protection that is afforded to Alan. If this is the case, then, this example becomes much less straightforward, in that Betty's action is both an interference that requires justification and a liberty that is presumptively protected, and that she is under no obligation to justify.

This result would apply to many other situations. If Alan reacts to Betty's action, for example, by struggling free from her grip, then this would be both a protected and an interfering action, as would any subsequent physical response from Betty. In fact every interfering action is also a protected action. This seeming contradiction might be overcome by saying that the requirement to justify interference rebuts the liberal presumption, but this raises an even more disturbing possibility: that Alan's action of splitting the pebbles amounts to an interference on Betty. It is possible to imagine that Betty suffered some harm as a result of Alan's splitting of pebbles. That harm would seem inconsequential, but it is no more so than the harm caused by her action against Alan. If there is some harm involved, there seems no obvious and logical way to distinguish it from the harm then suffered by Alan, and, so, Betty would be entitled to some sort of justification according to Gaus' formula. This result would be entirely unacceptable to Gaus, as it paints his liberal principles as requiring a complex and cumbersome web of harm and justification.

Gaus might respond to this argument by claiming that Alan does not actually harm Betty, or that any harm suffered is not of the relevant kind, so that his action does not interfere with any protected liberty. The possibility of harm, in the sense of having a significant negative effect on Betty, is real, and would make sense of her behaviour. This harm could take many forms, but the most reasonable in the unusual set of circumstances is a sense of indignation. Gaus might argue that this does not constitute the kind of harm that could amount to an interference, and it is correct that there is a trend in liberal theory to exclude such moralism-like harms. Yet this would require that Gaus actually offer an idea of what amounts to interference and what does not.

The point is that Gaus' example is supposed to show one person carrying out an inconsequential act and another person interfering with that act. By paring back this interaction, the quintessence of liberalism should be revealed. Further examination, however, suggests that the conceptions of protection and interference are not easily 
understood or applied, even in the most basic example, and this raises questions about such a one-sided situation. In fact, it is possible that the liberal asymmetry Gaus identifies is actually a consequence of a misshapen circumstance. The difficulty with his approach is that the conceptions of protection and interference are so broad as to apply to almost any situation, so that, in any social interaction where actions affect others, every action is both protected and is an interference. Gaus' example relies on an inconsequential action on one part and a motiveless response on the other, expressed as a direct physical harm. Instead of distilling a social situation into pure liberal rights, it serves only to codify an unreal situation for general use. Gaus seems to rely more on the liberal intuitions of the reader to make the link between this situation and general social life, than on the effective characterisation of the liberal conceptions involved.

\subsection{Public reason in liberalism}

Public justification is an important post-communitarian thread of contemporary liberal theory, but it is not the only thread; it does not, by itself, express what is fundamental in liberal theory, nor does it offer an obvious and universal justification for a public reason liberalism. Both Gaus and Waldron do, however, elucidate the reasons why such forms of liberalism are so compelling: They require social principles and forms to be justifiable to each person, and, in this way, public justification liberalism taps into the powerful liberal and moral ideals of consent and respect. However, as both Gaus and Waldron admit, actual consensus is not a realistic or even a desirable goal. A conception of public justification must respond to this in a sensible way, while retaining as much moral content as possible.

A liberal theory of public justification draws on the deep and rich moral sources of consent and respect, yet must assert itself as the proper or fundamental expression of liberalism, or as the best system of social organisation. This burden can be obscured by the centrality of justification to such theories. All moral and political theories face justificatory burdens; the requirement of giving reasons or a rational explanation for a particular approach is fundamental. Public justification differs in that it implies that 
this justification is or could be made to each person on their own terms, giving reasons that they would accept, and that this is carried out or confirmed through an actual public, political procedure.

This could be contrasted with A Theory of Justice. There, the basis for justification in the broadest sense is also agreement or consent, in the form of a reflective equilibrium, but this differs in that the agreement is reached through personal reflection rather than a public process of dialogue. It is based on going through a particular theoretical and rational exploration of the original position, rather than justifying according to existing beliefs, mitigated by the limitations placed on, or virtues required of, those who engage in the public process. Because both approaches depend on agreement, and, so, on intuition based justifications, there are substantial similarities between them. They differ in that A Theory is contemplative and theoretically structured, where public justification is based on discourse and justification by good reasons.

The contrast is greater between political justification and a non-intuitive political theory, such as in Anarchy, State, and Utopia. With the latter, justification is through a legitimacy standard based on a declared set of natural rights. There, the justification is not supposed to reflect individual beliefs at all. As such, coercive political rules, such as are in a libertarian theory, are applied despite their disagreement with personal beliefs or intuitions. This process of justification is quite different in terms of a greater theoretical content, and is not explicitly public.

From these comparisons it is clear that public reason offers a different and potentially powerful justification. It draws on the values of consent and respect, and incorporates a diversity of beliefs within a single unified political system. In this way public reason is better equipped than the systems, outlined in, either, A Theory of Justice, or, Anarchy, State, and Utopia, to deal with moral complexity and contestability. While accepting that beliefs are plural and that actual agreement is difficult to achieve, public justification allows a standard by which consensual or quasi-consensual decisions can be reached, or at least provide an acceptable basis for a democratic result. Also, by applying some useful distinction between the public and the private, such that some aspects of belief are consigned to the private, some of this difference 
can be overcome. In this way, public justification allows some contestability and complexity to be dealt with within the domain of the political, that is, when removed from its theoretical structure. It also benefits from the virtues of a discourse-based approach to morality, although the effect of these differs with different theorists. Finally, the approach allows a theory to be silent on substantive political principles, and allows, instead, a particular society to choose from a plurality of alternatives depending on particular circumstances and beliefs. In this way, the difficulty of deriving substantive principles from universal theoretical structures without incorporating a theorist's particular beliefs is diminished.

However, public justification also faces a number of challenges. As is evident when considering the arguments of Waldron and Gaus, the idea of public justification is not self-justifying and, instead, must form part of an underlying theoretical justificatory structure. This reliance on theory is not contrary to public justification per se, but a theory of public justification needs to explain the moral role of dialogue and intuition within the theory, and also to relate this to the analytical theorising. Since these all form necessary parts of such a theory, and, yet, in some sense, compete in founding substantive principles, it is important that their relationship is understood. Gutmann and Thompson, for example, clearly prefer a system whereby a close analysis of intuitive liberal values informs democratic deliberation. Theorist Stephen Macedo places public justification in a representative role:

\footnotetext{
mediating, in effect, between philosophy and the citizenry (representing philosophy to citizens and citizens to philosophy). The public conception avoids excessively subtle and complex forms of reasoning.... Public justification respects the "constraints of simplicity and availability of information," moderating the aims of philosophy so as to ensure the wide accessibility of the relevant forms of reasoning and evidence. ${ }^{155}$
}

This does not necessarily mean a dumbing-down of public discussion to the point where the complexity of the subject matter involved is not adequately reflected. It does recognise, however, that public justification means justification on the terms of the public, and, so, would exclude some of the rarefied arguments of the academic philosopher. This downplaying of philosophy in determining substantive moral and political issues is important and needs to be supported within a theory of public

${ }^{155}$ S Macedo, 'The Politics of Justification' (1990) 18(2) Political Theory 280, p283. 
justification, whether by arguments of subjectivism, scepticism, the power of public dialogue, or in some other way.

In this way, liberal public justification faces the full justificatory burden, as does any moral or political theory. In addition, there are two more practical problems. The first relates to agreement: there is a fundamental tension in public justification theories between the values of consent and respect on which they are based, and the context of persistent disagreement in which they operate. Consent and respect, in this context, imply that individuals are accepted in whatever beliefs they hold, and are in substantive agreement with each political outcome. But, actual consensus is unlikely to ever result on this level, especially in the context of an irreducible plurality of beliefs.

Any liberal theory of political justification, then, must have a conception of justification that allows continuing disagreement while still meeting, in some sense, the standards of consent and respect. This conception must be sufficient to ensure the level of agreement that is aimed at can be achieved. This usually means incorporating an understanding of reasonableness that limits political consideration. Such an inclusion is important in order to exclude those who have fundamental differences in beliefs, such as the deeply illiberal. That this skews political justification is a simple fact of such theories, and encourages contextualised justifications based on, at the very least, a desire to co-operate in the political domain. ${ }^{156}$

The second practical difficulty in liberal public justification theories lies in actually incorporating substantive liberal content, or in ensuring that basically liberal results would come from the process of public justification. Some basic liberal values, such as the right to free thought and speech, and to be free from certain types of discrimination, will be included as being necessary to public justification itself. Other liberal content, however, is much more difficult to incorporate. This can be seen clearly in Gutmann and Thompson's strained attempts at justifying principles of liberty and opportunity as being necessary to deliberation; in Rawls' attempt to justify

${ }^{156}$ See, for example, ibid, p289. 
the original position as the most reasonable political conception; and, in Gaus' arguments as to conclusive liberal justifications.

This difficulty concerns the potential for conflict between substantive liberal principles and the undetermined and open nature of the process of political justification. The latter is supposed to be attuned to the beliefs of particular people, and, as such, should offer no guarantee that liberal outcomes will be reached. This puts liberal public justification theorists in the position of placing liberal restrictions on the political process (similar to Gutmann and Thompson), assuming a pre-existing liberal or democratic culture (Rawls and Waldron), or arguing that the process of justification itself would naturally lead to liberal results (Gaus). Of these, the most plausible is the contextual approach as employed by Rawls; but, this approach justifies public justification only to those who are already predisposed to accept it, and suffers some of the problems that go along with a contextual justification.

At the most general level, public justification resembles pluralism, in that both seek to derive a liberal system out of conditions of diversity or difference. The basis for each is to develop a plausible conception of social difference, be it value pluralism or reasonable disagreement, and embed it into a general liberal system. This in itself resembles Rawls' earlier subordination of differing conceptions of the good to the right. Each of these systems faces a strong justificatory burden that cannot be satisfied simply by the plausibility of the difference it contains. This entails that the nature of the difference is such that it does not overwhelm any attempts to place it within a singular system, so that the system itself can be justified in the face of the persistent and irreducible nature of the difference.

As was explored in the chapter on A Theory, the distinction between the right and the good is not itself adequate to support such an approach: rational disagreement is not confined simply to conceptions of the good, as the criticism of Rawls' theory shows; and, the two are not so distinct as to justify Rawls' assignation of priority to the relationship between them. Theories of pluralism or public justification that adopt this distinction will always suffer from this weakness. Equally fraught is the thoroughgoing approach that sees pluralism as the central fact of moral reality. Any theory based on this conception will face difficulty in being able to define a unified 
system that could overcome this fundamental pluralism: the fact of pluralism is itself singular, but a political and moral theory cannot be derived wholly from this fact.

Theories of public justification (and political liberalism) approach this problem by being concerned with the actual social fact of disagreement, and by operating through a public and political process. This allows the embedding of a social and public difference into a theoretical system, and, so, permits a sensible distinction on those grounds. This is an attractive approach in that the division between the social and the theoretical is real and readily recognisable. The difficulty is that, because the theories are necessarily intuition-based, the political is not capable of being separated from the philosophical in this way, and, so, the required distinction between philosophical theorising and public reasoning breaks down. This leads to internal inconsistency, and the difficulty of justifying theoretical systems as wholes.

These attempts by pluralism, and by political liberalism and public justification theory, are essentially to incorporate fundamental contestability and complexity into political theory in the form of embedded conceptions of difference. None of these have been successful in creating a morally meaningful and coherent theory of justice, for the general reason that none has been able to restrict difference to within a generally justifiable system. 


\section{MULTICULTURALISM}

\section{Liberal theory and group rights}

\subsection{The multicultural movement}

Multiculturalism as a political ideology originates from the 1960s, and in its most extreme form

expresses a reservoir of alienation against the cultures, ethnicities, religions and mores of Europe and of peoples who derive their origins from Europe ... while championing the perspectives and ways of life of non-European peoples. ${ }^{1}$

The subjects of this alienation are those who are not, or who do not identify with, the Euro-central perspective and, instead, who embody some aspect of separation from this norm. A consequence of the multicultural movement is that

previously excluded groups are no longer willing to be silenced or marginalized, or to be defined as 'deviant' simply because they differ in race, culture, gender, ability or sexual orientation from the so-called 'normal' citizen. They demand a more inclusive conception of citizenship which recognizes (rather than stigmatizes) their identities, and which accommodates (rather than excludes) their differences. ${ }^{2}$

These demands for multiculturalism have had a major impact on liberal theory from the late 1980s. The first expression of this impact viewed multiculturalism as a particular expression of the communitarian critique of liberalism. ${ }^{3}$ One of the communitarian criticisms concerns the liberal assumption of the autonomous and unencumbered self, free to choose and pursue its own conception of the good life. Multiculturalism represents a challenge to this idea in that it implies a conception of the self that is dependant on and expressed through a particular culture, and suggests that the liberal self is, in fact, a western self, whether in the details of construction or through the way it is assumed to act.

\footnotetext{
${ }^{1}$ D D Murphey, “"Multiculturalism” and the West' (2005) 30(2) Journal of Social, Political, and Economic Studies 203, p203.

${ }^{2}$ W Kymlicka, Contemporary Political Philosophy ( $2^{\text {nd }}$ edn., 2002), p327.

${ }^{3}$ Ibid, pp336-337.
} 
The second, and more significant, impact consists of the acknowledgment of the importance of multiculturalism to liberal theory, and the attempt to incorporate into liberalism some conception of multicultural or group rights. Contemporaneous with many of the developments discussed in the previous two chapters has been attempts to develop a liberalism consistent with the real-world fact of multiculturalism, and, so, a more realistic and culturally richer system, able to incorporate these rights. This liberal attempt at internalising multiculturalism has led the prominent multicultural liberal theorist Will Kymlicka to comment that the "overwhelming majority of debates about multiculturalism are not debates between a liberal majority and communitarian minorities, but debates amongst liberals about the meaning of liberalism". ${ }^{4}$

This incorporation is interesting and relevant in a number of ways. In terms of the two previous chapters, multiculturalism offers an alternative and potentially complementary perspective on difference, so that multicultural liberalism represents a new attempt at a liberalism that is derived from, or that at least has to deal with, fundamental variations in beliefs and values. How this difference is embedded within a liberal theory, and how this differs from the previous approaches, is important, as are the particular justificatory challenges that such a theory faces.

There have been a number of different approaches to this problem. In particular, some thinkers attempt to simply incorporate group rights seamlessly into a clearly liberal framework, while others reshape liberalism itself in order to cohere with multicultural ideas. Both of these approaches offer a potentially important development of liberal theory as a response to difference.

\subsection{The politics of recognition}

Charles Taylor's essay, 'The Politics of Recognition' (1994), represents the first steps towards a theory of multicultural liberalism, and reveals some of the challenges

\footnotetext{
${ }^{4}$ Ibid, p338.
} 
involved. ${ }^{5}$ Despite being recognised as a communitarian thinker, Taylor presents a distinctively liberal approach, albeit one strongly linked to the ideas of recognition and identity.

For Taylor, multiculturalism is founded on the more general demand for the social recognition of one's identity, meaning their self-conception. A failure to offer this recognition amounts to a real and, in fact, major harm.

The thesis is that our identity is partly shaped by recognition or its absence, often by the misrecognition of others, and so a person or group of people can suffer real damage, real distortion, if the people or society around them mirror back to them a confining or demeaning or contemptible picture of themselves. Nonrecognition or misrecognition can inflict harm, can be a form of oppression, imprisoning someone in a false, distorted, and reduced mode of being. ${ }^{6}$

In this way, society can, independent of any particular human rights it recognises, inflict harm on groups of people by failing to recognise those groups' identities as distinct from their own. This harm can amount to a "grievous wound", such that due recognition is a "vital human need". 7 The importance of recognition is heightened in modern times, Taylor argues, because of our understanding of an individualised identity that is inward-looking and subjective. ${ }^{8}$

By attaching to it such social importance, Taylor creates a conception of recognition that is highly relevant to matters of justice. In explicating this idea, he draws a distinction between the ideal of equal recognition and the universal theories of equal dignity. The latter refer to the earlier liberal theories of justice that are supposedly universal, and that prescribe for every person a standard bundle of rights. ${ }^{9}$ By contrast, equal recognition represents a politics of difference; it acknowledges distinctness between people, and rallies against any homogenisation and assimilation. The former is based on an idea of equal respect for all and, in particular, Taylor argues, on a universal human potential. The politics of difference, however, looks not to potential but to actuality, and seeks protection for what individuals and cultures

\footnotetext{
${ }^{5}$ Taken from an earlier (1992) essay, 'Multiculturalism and "The Politics of Recognition", published as the centrepiece to C Taylor, Multiculturalism: Examining the Politics of Recognition (1994).

${ }^{6}$ Ibid, p25.

${ }^{7}$ Ibid, p26.

${ }^{8}$ Ibid, pp28-29.

${ }^{9}$ Ibid, p38.
} 
have actually achieved. ${ }^{10}$ This leads to a necessary conflict between the two approaches.

For one, the principle if equal respect requires that we treat people in a difference-blind fashion.... For the other, we have to recognize and even foster particularity. The reproach the first makes to the second is just that it violates the principle of nondiscrimination. The reproach the second makes to the first is that it negates identity by forcing people into a homogeneous mold that is untrue to them. ${ }^{11}$

Taylor goes on to explain how this mould is not neutral between different people and different groups of people, but may favour one hegemonic group. In this way, a seemingly liberal approach has the potential to be discriminatory. ${ }^{12}$

Taylor responds to the tension between these approaches by moving away from the idea of liberal neutrality, and, instead, allows that a liberal state can aim at a particular set of collective goals. Such a state remains liberal when these goals respect the diversity of citizens, particularly those who are not members of the group amongst whom the goals are shared. ${ }^{13}$ For a particular ethnic minority this may mean recognising a substantial set of specific, group-based rights, but these would not negate or alter basic individual rights. ${ }^{14}$

There would be no question of cultural differences determining the application of habeas corpus, for example. But [models of liberal society based on the politics of difference] distinguish these fundamental rights from the broad range of immunities and presumptions of uniform treatment that have sprung up in modern cultures of judicial review. They are willing to weigh the importance of cultural survival, and opt sometimes in favour of the latter. They are thus in the end not procedural models of liberalism, but are grounded very much on judgments about what makes a good life - judgments in which the integrity of cultures has an important place. $^{15}$

This means that Taylor does not construct a particular justified liberal system. His explanation extends only to a basic description of the interaction between culturebased and individual rights: that fundamental individual rights will prevail, but that group rights could trump lesser or secondary individual rights when the needs of the particular culture outweigh the value of these rights. In this situation, the weight given to these group rights must be informed by the needs of recognising the identity the culture offers.

\footnotetext{
${ }^{10}$ Ibid, pp41-43.

${ }^{11}$ Ibid, p43.

12 Ibid.

${ }^{13}$ Ibid, p59.

${ }^{14}$ Ibid, p61.

${ }^{15}$ Ibid.
} 
Not all minority cultures, Taylor admits, would be able to be accommodated by this distinctively liberal regime, and, so, the challenge is to deal with diversity while remaining essentially liberal. ${ }^{16}$ This should be met by employing a presumption that all cultures are owed equal respect. This is not to say that all cultures are equally worthy, but that "all human cultures that have animated whole societies over some considerable stretch of time have something important to say to all human beings". ${ }^{17}$ It is difficult to find a clear foundation for this presumption, but the mere success of such a culture, Taylor suggests, implies something that is worthy of admiration, and that it would be arrogance to claim otherwise. ${ }^{18}$

Despite failing to offer a substantive theoretical structure, Taylor's work indicates some key issues for liberal theories that seek to incorporate multiculturalism. Probably the most significant of these is the tension between individual and group rights. The liberal preference for rights that are universal and, so, reflect a credo of equal respect and equal treatment, does not sit well with the recognition of rights that apply to a particular cultural group. For this reason, the initial task of any multicultural liberalism is to explain why and how individual rights are not adequate. For Taylor, this is because such rights do not fully recognise the identities of minority cultures and, instead, reflect a system biased in favour of the dominant culture.

Chandran Kukathas, himself a libertarian theorist, disagrees with this position. He argues that liberalism should resist any minority claims for recognition, and that individual rights are sufficient to ensure that all are treated fairly. ${ }^{19}$

The reason why liberalism does not have a problem with multiculturalism is that liberalism is itself, fundamentally, a theory of multiculturalism. This is because liberalism is essentially a theory about pluralism; and multiculturalism is, in the end, a species of pluralism. Liberalism is one of the modern world's responses - indeed its most plausible response - to the fact of moral, religious, and cultural diversity. Its response has been to say that diversity should be accommodated, and differences tolerated; that a more complete social unity, marked by a uniform and common culture that integrates and harmonizes the interests of individual and community, is unattainable and undesirable; that division, conflict and competition will

\footnotetext{
${ }^{16}$ Ibid, pp62, 64.

${ }^{17}$ Ibid, p66.

${ }^{18}$ Ibid, pp72-73.

${ }^{19}$ C Kukathas, 'Liberalism and Multiculturalism: The Politics of Indifference' (1998) 26(5) Political Theory 686, p687.
} 
always be present in human society, and the task of political institutions is to palliate a condition they cannot cure. ${ }^{20}$

Kukathas argues that a liberal system of individual rights is adequate to deal with issues of cultural pluralism, just as it deals fairly with other forms of pluralism; that the purpose of liberalism is to provide equal rights that allow each person to have a fair call on society's goods regardless of their personal beliefs, or the general acceptance of their cultural practices. On this view of liberalism, group recognition is unnecessary at best. At worst, it means the illegitimate promotion of a particular cultural group to the detriment of others, and has the potential to descend into "the politics of interest group conflict". ${ }^{21}$ Kukathas offers, instead, the view of a liberalism that can embrace a wide range of cultures, excluding only those that are irreducibly collective or illiberal. ${ }^{22}$ This is characterised by the spirit of neutrality, which is accepting of these different ways of life, and by entrenched rights of free association. $^{23}$

Taylor and Kukathas offer two quite different accounts of the proper liberal response to cultural pluralism. The essential difference between them seems to stem from the view each holds as to the power of liberal neutrality to accommodate cultural difference. Taylor holds that liberal conceptions of neutrality and equality are culturally biased, and that the recognition of cultural identities requires specific group rights. To Kukathas, liberal neutrality and individual rights, while not perfect, amount to the best response to cultural pluralism.

Determining which approach is correct requires a deeper examination of the meaning of liberal multiculturalism, but this difference does raise two issues. The first is whether difference-blind liberalism really contains cultural bias. Earlier discussion of neutrality- and equality-based liberalism identified the presence of bias, but in relation to the particular theorist rather than an entire culture. This does suggest that liberal theory cannot achieve a political and moral structure without implicit assumptions as to value. Many, more recent, theories of liberalism are contextually justified, and depend upon specifically western social conditions. Here, it seems, the bias is

\footnotetext{
${ }^{20}$ Ibid, p690.

${ }^{21}$ Ibid, pp692-693.

${ }^{22}$ Ibid, p695.

${ }^{23}$ Ibid, pp696-697.
} 
explicitly culturally identified, albeit the conditions are usually expressed in broad terms. If this bias is unavoidable in liberal theory, then multicultural liberalism seems meaningful as an expression of the idea that it may be culturally founded. In determining how liberalism is best able to respond, some account of the nature of liberal cultural bias, more extensive than Taylor's, will be required.

The second issue raised relates to the nature of multiculturalism itself. As Kukathas suggests, multiculturalism is a form of pluralism. As such, he argues that it should be treated like any other source of difference within a society. Multicultural liberalism contends, in contrast, that cultural pluralism is a special form of pluralism that requires that particular measures be incorporated into liberal systems. This is not quite the case with Taylor, who is concerned with misrecognition generally, whether for reason of cultural or other difference. Generally speaking, however, multicultural recognition needs to offer an account of the nature of cultural difference, and the corresponding shortcomings of difference-blind liberalism, in order to justify a particularly multicultural conception of liberalism.

\section{Multicultural Citizenship}

\subsection{Group rights as liberal rights}

Taylor's essay on multiculturalism does not amount to a settled and justified theory, but, rather, offers some of the reasoning that informs the need for a liberal theory that incorporates multicultural concerns. He envisages a liberalism that is sensitive to the recognition of people's identities, but one that does not override other fundamental liberal concerns, and, instead, is weighed against secondary rights. Will Kymlicka takes on the similar task of incorporating multiculturalism into existing liberal ideals, and his book, Multicultural Citizenship: A Liberal Theory of Minority Rights (1995), probably represents the best-constructed and most complete attempt at this approach. $^{24}$

\footnotetext{
${ }^{24}$ W Kymlicka, Multicultural Citizenship: A Liberal Theory of Minority Rights (1995).
} 
The central theme of Multicultural Citizenship is that rights that properly belong to minority groups, or members of minority groups, are not adequately expressed by the traditional set of human rights; and that, what is required, instead, is a system that recognises certain group rights, but that does not do so at the expense of its liberal core. ${ }^{25}$ Kymlicka undertakes this task by, firstly, identifying two common types of minority groups and the group rights that they might attract and, then, by justifying the operation of these rights within a liberal structure, in a way that is consistent with individual rights.

Kymlicka labels the two types of minority groups as ethnic and national minorities. A national minority pertains to a "historical community, more or less institutionally complete, occupying a given territory or homeland, sharing a distinct language and culture" within a state. ${ }^{26}$ This includes various indigenous peoples, as well as other settled nation-like communities (e.g. Quebecois in Canada). Ethnic minorities are established as a result of the voluntary immigration of members of a cultural group into a state. Traditionally, this was characterised by assimilationist policies on the part of the state, but, more recently, a more tolerant and pluralist approach has been adopted. ${ }^{27}$

Kymlicka also identifies three types of group rights that can legitimately be claimed by such minorities. The first relate only to national minorities and encompass the rights of self-government. ${ }^{28}$ These usually involve "devolving political power to a political unit substantially controlled by the members of the national minority, and substantially corresponding to their historical homeland and territory". ${ }^{29}$ The second type are polyethnic rights, and, so, are relevant to ethnic minorities. These include the funding of cultural practice and the promotion of cultural expression. ${ }^{30}$ Finally, special representation rights are appropriate to both types of minority, and involve the reserving of some legislative membership for the representatives of particular

\footnotetext{
${ }^{25}$ Ibid, pp4, 6 .

${ }^{26}$ Ibid, p11.

${ }^{27}$ Ibid, p14.

${ }^{28}$ Ibid, p27.

${ }^{29}$ Ibid, p30.

${ }^{30}$ Ibid, p31.
} 
minority groups. ${ }^{31}$ These are, Kymlicka argues, the basic mechanisms by which a minority can be accommodated within a liberal state, and are currently used throughout the democratic world. ${ }^{32}$ Kymlicka justifies the recognition of these rights within a liberal framework in two complementary ways. He argues, firstly, that culture is fundamental to the liberal freedom to choose the way one desires to live; and, secondly, that any liberal system is implicitly biased against minority cultures. In this way, both freedom and equality must be sensitive to culture in order that fair results are reached.

A societal culture, Kymlicka states, is one that "provides its members with meaningful ways of life across the full range of human activities, including social, educational, religious, recreational, and economic life, encompassing both public and private spheres". ${ }^{33}$ Such cultures, he argues, are fundamental to individual choice in that they provide options as to how to live, and make those options meaningful. ${ }^{34}$ They operate as the subject and as the context of choice, and language is an important aspect of the latter.

\begin{abstract}
Whether or not a course of action has any significance for us depends on whether, and how, our language renders vivid to us the point of that activity. And the way in which our language renders vivid these activities is shaped by our history, our 'traditions and conventions'. Understanding these cultural narratives is a precondition of making intelligent judgements about how to lead our lives. In this sense our culture not only provides options, it also 'provides the spectacles through which we identify experiences as valuable. ${ }^{, 35}$
\end{abstract}

Kymlicka sets as a precondition to the meaningful exercise of our freedom to choose how to live, the adherence to a societal culture. It is not simply the case, however, that a minority member can switch to a majority culture. Rather, the pull of one's own culture is strong, and any attempt to impose an alternative strongly resisted. ${ }^{36}$

Kymlicka concludes from this that individual freedom requires access to one's own societal culture. ${ }^{37}$ This access is guaranteed by the rights of self-government enjoyed by national minorities under his theory, who are then able to manage the access to and

\footnotetext{
${ }^{31}$ Ibid, p32.

32 Ibid, p33.

${ }^{33}$ Ibid, p76.

${ }^{34}$ Ibid, p83.

${ }^{35}$ Ibid. The quote is from R M Dworkin, A Matter of Principle (1985), p228.

${ }^{36}$ Ibid, pp86-90.

${ }^{37}$ Ibid, p101.
} 
development of the societal culture. There are two points that Kymlicka makes to clarify this result. The first is that the promotion of a particular culture does not interfere with a person's right to choose. This is because

[t]he freedom which liberals demand for individuals is not primarily the freedom to go beyond one's language and history, but rather the freedom to move around within one's societal culture, to distance oneself from particular cultural roles, to choose which features of a culture are most worth developing, and which are without value. ${ }^{38}$

Kymlicka argues that an increase in the sensitivity to culture does not mean dulling the sensitivity to freedom, and that, on reflection, a focus on culture is fundamental to, and can enhance, freedom. The second point is that this approach does not prescribe cultural isolation. Having access to one's minority societal culture does not prohibit or prevent cultural interaction and intermingling. Even in the case of a national minority, there will generally be interaction between small self-governing minorities and their majority counterparts. ${ }^{39}$

Kymlicka's second argument in favour of group rights stems from the same assertion of liberal cultural bias that is central to Taylor's essay.

Government decisions on languages, internal boundaries, public holidays and state symbols unavoidably involve recognizing, accommodating, and supporting the needs and identities of particular ethnic and national groups. The state unavoidably promotes certain cultural identities, and thereby disadvantages others. ${ }^{40}$

In this way, Kymlicka argues, one societal culture is preferred by the state over another. This means that adequate access to one's culture requires redressing the balance, and specifically acknowledging and promoting other cultures. He expresses this argument in terms of a conception of equality for individuals: that the equal treatment and respect of all people requires group-specific rights that act to correct for the inevitable cultural bias of the state. It is significant not only that he taps into such a fundamental liberal concern as equality, but also that he justifies group rights on an individual basis - not because of the rights or interests of an ethnic or national group, but, rather, those of its members.

\footnotetext{
${ }^{38}$ Ibid, pp90-91.

${ }^{39}$ Ibid, pp103-104.

${ }^{40}$ Ibid, p108.
} 
Kymlicka contrasts this approach with the more traditional liberal position that he labels benign neglect. ${ }^{41} \mathrm{He}$ argues that despite the attraction of this position, equality and fair treatment sometimes require that a cultural perspective be adopted, and that corrective group rights be employed. ${ }^{42}$ As to the proper effect of these measures, Kymlicka asserts that "we should aim at ensuring that all national groups have the opportunity to maintain themselves as a distinct culture". ${ }^{43}$ This equality argument applies also to ethnic groups, but in a different way. Because immigration is undertaken with the acceptance of the need to integrate, to some extent, into the new culture, arguments as to cultural bias are less relevant. ${ }^{44}$ Instead, equal concern requires that 'good cultural membership' be promoted to the immigrants, which involves "enabling integration, by providing language training and fighting patterns of discrimination and prejudice". ${ }^{45}$ In doing so, the majority should attempt to accommodate the minority culture. As the earlier point about switching cultures infers, integration can be neither automatic nor total. ${ }^{46}$

Kymlicka offers three additional arguments to buttress his justification of group rights, but none of these are as significant as the argument from equality and cultural bias. These concern, firstly, the significance of historical agreements as to the sharing of national and cultural rights, as are present in some countries where western settlement has affected established indigenous groups. ${ }^{47}$ The second asserts the value of cultural diversity. Like the arguments in favour of value diversity canvassed in the chapter on pluralism, this has some potential value but is not well-developed. Kymlicka asserts that intercultural diversity "contributes to the richness of people's lives". ${ }^{48}$ He goes on to examine the quasi-aesthetic and practical advantages of such diversity, but concludes that it is not an effective argument, since diversity cannot distinguish between chosen and enforced difference. ${ }^{49}$

\footnotetext{
${ }^{41}$ Ibid.

${ }^{42}$ Ibid, p109.

${ }^{43}$ Ibid, p113.

${ }^{44}$ Ibid, p114.

${ }^{45}$ Ibid.

${ }^{46}$ Ibid, p115; see above, note 36.

${ }^{47}$ Ibid, p116.

${ }^{48}$ Ibid, p121.

${ }^{49}$ Ibid, pp121-123.
} 
Finally, Kymlicka argues that one of the functions of states is to protect the societal culture of its members, and that the national minority situation is analogous to the state system. Consequently, national minorities should have powers of cultural protection. ${ }^{50}$ This argument concludes Kymlicka's justification for group-specific rights. Primarily this is undertaken through asserting the fundamental importance of culture to individual choice and action, and that any fair and realistic conception of equality must be conscious of cultural bias inherent in political practice. This prescribes, depending on the particular circumstances, various self-government rights for national minorities, and rights of fair integration and reasonable accommodation for ethnic minorities. Kymlicka completes his theory of multicultural citizenship with two further points. The first sets out how the rights of self-government granted to national minorities relate to individual liberal rights, and the second responds to a criticism of multicultural liberalism, that it would undermine the unity and stability of a society.

The self-government rights of national minorities imply the power of such a group to guide the lives of its members. Kymlicka uses a distinction between external protections and internal restrictions to mediate the interaction between group and individual rights. Internal restrictions refer to limitations placed on individual action through national minority government. Liberalism suggests that these should not limit the basic rights afforded to individual members of the group, but Kymlicka argues that liberal states should not impose forcibly rules on minorities, except in extreme circumstances. $^{51}$

Conversely, external protections represent the limitation of the power of the cultural majority over the minority group. A group's claim for external protection will be legitimate insofar as these encourage equality between adherents to the minority and majority cultures, "rectifying disadvantages or vulnerabilities suffered by the members of a particular group". 52 In this way, Kymlicka argues that a national minority is able to avoid the influence of the majority population so long as this reflects an underlying equality of treatment and respect for the members of both

\footnotetext{
${ }^{50}$ Ibid, p125.

${ }^{51}$ Ibid, pp165-167.

${ }^{52}$ Ibid.
} 
groups. In practice this will mean that the minority is able to avoid the culturally homogenising power of the majority, to the extent that its members are inclined to retain their own culture. However, the minority will not be able to impose its own culture on unwilling recipients, as this would undermine their individual liberties. The resulting system, whereby a national minority has the capacity to protect its culture, subject to basic human rights, Kymlicka describes as "impeccably liberal". 53

Finally, Kymlicka confronts the argument that such group rights would undermine the unity of a liberal society. He argues that, in relation to ethnic minorities, this is an overstated threat: immigrant populations have generally demonstrated a willingness to integrate, and rights which recognise this, while prescribing reasonable accommodation of the minority, will not weaken the social fabric.

To be sure, [immigrants] want the mainstream institutions in their society to be reformed, so as to accommodate their cultural differences, and to recognize the value of their cultural heritage. But the desire for such polyethnic rights is a desire for inclusion which is consistent with participation in, and commitment to, the mainstream institutions that underlie social unity. $^{54}$

Conversely, the rights of self-government that attach to national minorities do imply a weakening of the bonds of the broader society. This is a significant concern, Kymlicka argues; but, the refusal of such rights to a national minority may well alienate them further and deepen the desire for formal separation. ${ }^{55}$ Ultimately, solidarity in a multinational state is achieved not by forced integration or the imposition of one group's culture on another, but by the accommodation of minority groups and identities by the majority. ${ }^{56}$ The result is a state that is recognisably liberal, but that is sensitive to different cultural perspectives, and deals fairly with minority groups through an appropriate set of group rights.

\subsection{Culture and multiculturalism}

\footnotetext{
${ }^{53}$ Ibid, pp152-153.

${ }^{54}$ Ibid, p178.

${ }^{55}$ Ibid, p183.

${ }^{56}$ Ibid, p188.
} 
The nature of Kymlicka's theory is of culture-based additions to a non-cultural liberal framework: group rights are recognised as appropriate for some minorities for reasons that are not normally discussed in liberal theory, and that do not undermine its basic ideas. Like Taylor, Kymlicka is concerned with adjusting liberalism to take account of hitherto unregarded cultural concerns. The basic theme of such a project is that liberalism fails to account adequately for the cultural differences that are normal for contemporary western states. As is discussed above, in order to be effective, the theory must explain the nature of this failure and the difference in treatment of minorities that this implies.

The basis for this argument in Multicultural Citizenship is set out by Kymlicka in two parts: firstly, in the relationship between societal culture and freedom; and, secondly, in the liberal bias in favour of the majority, and the implications of this on equal respect and treatment. These considerations, he argues, favour a particular set of group-specific rights. At the heart of Kymlicka's account are conceptions of culture and multiculturalism. The relevant expression of the former is a societal culture that informs all aspects of a person's life. This has been criticised by a number of writers as being 'monolithic' and 'totalistic'. ${ }^{57}$ Kymlicka perceives culture to be relatively fixed, inescapable, complete and all-encompassing. This picture seems inaccurate, especially with regards to so-called national minorities that have suffered a history of marginalisation at the hands of the majority. ${ }^{58}$ It seems, also, to ignore the lesspernicious dynamic interplay between majority and minority cultures, and both intracultural diversity and cultural transformativity.

The way that Kymlicka conceives culture has significant consequences for his theory. As Christian Joppke has noted, there is

a tension in Kymlicka's concept of culture between being either too thick or too thin: 'too thick' for giving a realistic account of the relationship between liberal states and culture; 'too thin' for justifying any minority rights at all, particularly for immigrants. ${ }^{59}$

\footnotetext{
${ }^{57}$ C Joppke, 'Multicultural Citizenship: A Critique' (2001) 42(2) European Journal of Sociology 431, p436; M Akan, 'Contextualizing Multiculturalism' (2003) 38(2) Studies in Comparative International Development 57, p62.

${ }^{58}$ Joppke, op cit, above note 57, p436.

${ }^{59}$ Ibid.
} 
This passage suggests that Kymlicka is caught in a difficult position: if culture is, as he seems to assert, conceived thickly, then the portrayal of (national) multiculturalism will be unrealistic; it will anticipate a separate and complete minority culture that resists, not only, the urge to integrate, but, also, the necessity of being liberal. The alternative, Joppke argues, is of a thin culture whereby a minority culture would readily give way to both majority and liberal demands.

Kymlicka's response to this difficulty is to apply a thick societal culture to national minority groups, while arguing that ethnic minorities lack the "'institutional cement' needed to form or maintain a distinct societal culture" ${ }^{60}$ In this way, he can realistically maintain that national minorities can support self-government rights, while arguing that this would be inappropriate for immigrant communities. This is a clever move, and, with it, the criticism of societal culture collapses into what is probably the most persistently criticised aspect of Kymlicka's theory, namely the distinction between national and ethnic minorities.

However, Kukathas criticises this view of multiculturalism as being unrealistic, and the two groups as being, upon examination, somewhat indistinct. He identifies the voluntariness of being part of a minority as being the key point of distinction between the two groups, which seems an accurate portrayal of Kymlicka's account. Kukathas argues that this is not always the case in real life: many migrant populations are involuntarily so, and many indigenous peoples have the ability, at low cost, to exit their communities "and live as (cosmopolitan) members of the wider society." ${ }^{61} \mathrm{He}$ concludes that

national minorities and ethnic groups are not easily distinguishable - particularly if voluntariness of membership in the community or wider society is the yardstick. The effluxion of time increases the difficulty, as groups mingle, grow or contract; and as migrant generations find their ancestry more in the land of their birth than the homelands of their grandparents. ${ }^{62}$

In this way, Kukathas attempts to undermine the basis of Kymlicka's justification of group rights, namely that there are two different and distinct types of minority groups that draw different expressions of group-recognition, and instead declares "the

\footnotetext{
${ }^{60}$ Kymlicka, op cit, above note 24, p100.

${ }^{61}$ C Kukathas, 'Multiculturalism as Fairness: Will Kymlicka's Multicultural Citizenship' (1997) 5(4) Journal of Political Philosophy 406, p413.

${ }^{62}$ Ibid, p414.
} 
complexity and fluidity of cultural diversity". ${ }^{63}$ The criticism here is of the simplification, not of the idea of culture, but of the distinction between national and ethnic minorities.

This is not entirely fair to Kymlicka. In a reply to similar arguments, he points out that national and ethnic minorities together are not intended to exhaust the possible forms of minority cultures, but rather to be two distinct categories that are "the most common, the most successful, and the most relevant for future-oriented decisions". 64 Creating an approach relevant to these minorities would lead, Kymlicka suggests, to being able to deal with more difficult intermediate positions. ${ }^{65}$ This argument is an effective defence to the assertion that there are minority populations that do not fit easily into either definition, or that have the characteristics of both.

It would not, however, be adequate as a defence to the assertion that multiculturalism involves an inherent complexity of historical and cultural interaction that belies such a simple classification. Bernard Yack states that "Kymlicka's self-government rights cannot be granted to all legitimate claimants because the twisted history that has governed the distribution of land and population over the ages has hopelessly entangled their claims". ${ }^{66}$ Yack implies that, in real-life cases, the definition of a national minority, as occupying a distinct territorial, linguistic and cultural homeland, will often (even, usually) not be met. This would only be exacerbated by the monolithic conception of societal culture that is required for a group to be a national minority.

Kymlicka does undertake a simplification of culture and the forms of multiculturalism - the basis for national minorities depending on societal culture, and, for ethnic minorities, the voluntariness of minority membership. Any political theory based on an actual political movement as widespread and significant as that of multiculturalism will be forced to deal with the variation and complexity of particular historical situations. Whether Kymlicka's description is so simplified as to present a distorted

\footnotetext{
${ }^{63}$ Ibid, p416.

${ }^{64}$ W Kymlicka, Politics in the Vernacular: Nationalism, Multiculturalism, and Citizenship (2001), p59.

${ }^{65}$ Ibid, pp56-59.

${ }^{66}$ B Yack, 'Multiculturalism and the Political Theorists' (2002) 1 European Journal of Political Theory $107, \mathrm{p} 114$.
} 
and impoverished conception of multiculturalism, on which no general conception of group rights can be validly founded, remains to at issue. On theoretical terms, what is important is not that Kymlicka sets out a structure that could cover most circumstances of multiculturalism, but, rather, that he succeeds in showing a basis for a liberalism that is culturally sensitive. What is important is how Kymlicka's conceptions of culture and multiculturalism form part of, and, yet, potentially discredit, his justification of group rights. In assessing this justification, the effect of these simplifications should become clearer.

\subsection{Societal culture and liberal freedom}

The first part of Kymlicka's justification of multicultural rights stems from his conception of societal culture, and is primarily aimed at justifying rights of selfgovernment for national minorities. It rests on the assertion that access to one's societal culture is necessary for exercising liberal autonomy. The link between culture and choice is certainly deep and rich, but this result depends both on a strong view of culture, and on the importance of autonomy. Kymlicka clearly favours an autonomybased liberalism. In fact, Multicultural Citizenship has been criticised for giving autonomy too central a role, and, so, being based on a cultural value that would not necessarily be acceptable to other cultures. ${ }^{67}$ This latter point is an expression of the more general tension in multicultural liberal theory between particular cultures and approaches to liberalism, and will be addressed below. Although some questions remain about the content of Kymlicka's liberalism, his use of autonomy is so widely recognisable by liberals that it retains much of its power.

An argument often made against this justification of self-government rights, is that for the purpose of promoting autonomy, any societal culture will do; in other words, that autonomy would be satisfied by minority groups taking on the majority culture. ${ }^{68}$ Because culture is valuable relative to autonomy, Kymlicka's approach certainly seems susceptible to this idea. The problem with this is that, as Kymlicka states,

\footnotetext{
${ }^{67}$ C Knight, 'Liberal Multiculturalism Reconsidered' (2004) 24(3) Politics 189, p192.

${ }^{68}$ E.g. G Doppelt, 'Is There a Multicultural Liberalism?' (1997) 41(2) Inquiry 223, p230.
} 
people are attached to their culture, and it is very difficult to change. ${ }^{69}$ National minority groups, therefore, cannot be expected simply to switch culture in order to enjoy personal autonomy.

This is a successful defence of the suggestion, but it does not sit easily with the position in relation to immigrant communities. These groups, Kymlicka opines, should integrate into the majority culture. The different approach, he would argue, depends on the immigrants' willingness to integrate, as well as the fact that immigrant cultures are not societal cultures, as he understands them. What is disconcerting about the different results for national and ethnic minorities is that the former are predicated on the complete incapacity to shift cultures, and the latter on the ready ability to do so. This stark difference betrays Kymlicka's reliance on extreme and unrealistic minority categories. It is quite possible that immigrants tend to be more accepting of integration than indigenous populations, but the earlier criticisms suggest two distinct categories. In fact, as Joppke, Yack and others suggest, real political situations present much greater variation: where immigrants are unwilling to integrate, and, where indigenous groups do not possess the institutions and separation a societal culture requires. These are not, it is suggested, exceptions to Kymlicka's basic categories, but, rather, bespeak the complexity and variation in actual minority situations that defy this sort of concrete classification. If this is right, then Kymlicka's conception of separate polyethnic and self-government rights is not a common and useful framework, but, rather, a theoretical overstepping.

This criticism undermines the autonomy argument for group rights, on the grounds that this is based on an unrealistic picture of both societal culture and a national minority. Contrary to Kymlicka's assertions, it is quite possible that intercultural relations mean that the national minority group engages substantially and meaningfully in the majority culture; that they have no developed and separate cultural institutions; or, even, that the minority culture has ceased to have meaning as a separate culture. These possibilities weaken the reasoning between national minority status and self-government rights, and makes one wonder if the paradigmatic national minority exists at all.

\footnotetext{
${ }^{69}$ Above, note 36.
} 
Even if Kymlicka's self-government rights may not be generally applicable to national minorities in western countries, it may still have power as a theoretical argument. The issue, then, is how liberalism should treat this extreme example: the institutionally complete, physically separate, culturally and linguistically distinct community, found within a liberal state. Pre-multicultural forms of liberalism would suggest, simply, that the individual rights of the group members should be recognised. Kymlicka argues that this is inadequate, and that some rights of self-government should be granted, so as to perpetuate the separate culture. This, he argues, is necessary since their culture is fundamental to their choice. It is clear that this line of reasoning requires one more step, because applying the rules and principles of the liberal state would not permit adequate access to, or the "full and free development of", their culture. ${ }^{70}$ Practical realities aside, the success of Kymlicka's argument depends on him giving an account of how liberalism fails to meet these demands, and the effect of this on the ability of the individuals involved to exercise real autonomy. Kymlicka's monolithic conception of societal culture is also relevant here: it may be sufficient for autonomy that such people enjoy a mixed culture, or that such communities have a control over their cultures to an extent that falls short of selfgovernment. These matters seem bound up with Kymlicka's assertion of the cultural bias of liberalism, used in relation to the equality justification of group rights. These matters will be explored in the next section. Overall there does seem to be a potential link between group rights and liberal autonomy, but this is not adequately and generally explained by Kymlicka, and may not apply automatically to any actual examples of multicultural states.

\subsection{Minority self-government and individual rights}

If a liberal theory does grant to a national minority rights of self government, then the issue is raised of how those rights interact with the liberal rights recognised generally by the majoritarian state. In other words: how do culture and liberalism fit together in

\footnotetext{
${ }^{70}$ Kymlicka, op cit, above note 24, p27.
} 
relation to national minorities, the first of Kymlicka's two paradigms of liberal multiculturalism?

For Kymlicka these rights of self-government amount to external protections of the group's societal culture, and are supposed to be justified by the arguments from autonomy and equality. The relationship between these protections and the rules imposed by the group on its members is difficult. These rules can, potentially, limit the liberal freedoms that, as citizens, its members would otherwise enjoy. Kymlicka's position in relation to these so-called internal restrictions is not straightforward and, to properly understand it, requires the separation of two questions:

(1) what sorts of minority claims are consistent with liberal principles? (2) should liberals impose their views on minorities which do not accept some or all of these liberal principles? The first is a question of identifying a defensible liberal theory of minority rights; the second is the question of imposing a liberal theory. ${ }^{71}$

Answering the first of these, Kymlicka argues, involves identifying an appropriate and recognisable conception of liberalism - in his case, an autonomy-based liberalism. Any internal restrictions of national minorities would be contrary to such a conception, in that they restrict the individual autonomy of those involved; but, this does not mean that such restrictions should be prohibited. To the contrary, Kymlicka claims that, despite being clearly illiberal, these should be permitted in a selfgoverning minority unless they amount to a "gross and systematic violation of human rights, such as slavery or genocide or mass torture and expulsions". ${ }^{72}$

The reasoning behind this liberal restraint forms a crucial part of Multicultural Citizenship. Although it is not set out systematically, a number of different arguments are present in the text. Kymlicka suggests, firstly, that the liberal majority may not have the proper authority to intervene and reinstate the rights. ${ }^{73}$ It is not entirely clear what is meant by this, but it probably refers to the limited moral authority of the majority given the bias of liberalism and the respect owed the separate culture. Kymlicka follows this up by referring to the intuition that liberal rights should not be imposed on separate countries. ${ }^{74}$ This is an interesting claim, and he seems to explain

\footnotetext{
${ }^{71}$ Ibid, p164.

${ }^{72}$ Ibid, p169.

${ }^{73}$ Ibid, p165.

${ }^{74}$ Ibid.
} 
it by asserting the claim of the minority to self-government, and that the imposition of liberal rights may be seen as aggression. ${ }^{75} \mathrm{He}$ also argues that such attempts may backfire, and that liberalism only really works when its beliefs are internalised by the nation's citizens. ${ }^{76}$

These explanations are not without value, but they do not seem to establish sufficient grounds on which to base a central liberal principle of non-interference. The minority's perception and understanding of any imposition of liberal rights seem to be merely peripheral factors. This conception also suffers from Kymlicka's definition of societal culture: in such cases, the oppressed members of the minority may well reject this illiberal oppression. The picture of a single, total social culture offers no guidance as to what should happen when liberal beliefs are internalised by some of the minority nation's citizens. In addition, Kymlicka does not seem to allow that these internal restrictions actually diminish autonomy, so that the instrumental value of the societal culture is undermined by its illiberal content. This is a natural argument to make, and yet is potentially fatal to Kymlicka's assertion of the value of rights of selfgovernment to national minorities.

There is some value to Kymlicka's claim that simply imposing the political beliefs of a liberal majority on a separate minority culture can lead to greater harm than the approach of non-interference. However, this is inadequately explained in Multicultural Citizenship. In addition, it is expressed in a way that is inconsistent with its broader, autonomy-based structure. The incoherence of Kymlicka's argument for internal restrictions of self-governing national minorities is reflected in a number of basic and telling criticisms. For example, it has been criticised for: encouraging minority cultural homogenisation, despite this being contrary to the theory's most basic themes $;^{77}$ similarly, denying minority cultural development and evolution; ${ }^{78}$ and, also, allowing interference, without clear reason, in the case of extremely illiberal forms of internal restriction. ${ }^{79}$ Each of these criticisms comments on a cultural

\footnotetext{
${ }^{75}$ Ibid, p167.

${ }^{76}$ Ibid.

${ }^{77}$ Akan, op cit, above note 57, p64.

${ }^{78}$ Knight, op cit, above note 67, p193.

${ }^{79}$ Kukathas, op cit, above note 61, p420.
} 
conservatism that sits uneasily in a liberal theory committed to autonomy and cultural awareness.

\subsection{Culture and Equality}

If Kymlicka's project in Multicultural Citizenship is to attach cultural-based group rights onto a still-functioning liberal system, then an interesting contrast is provided by Brian Barry's book, Culture and Equality (2001). ${ }^{80}$ The latter is a wholesale criticism of projects of multicultural liberalism from the perspective of an egalitarian liberalism in the mould of the early Rawls. If Kymlicka's purpose is to align multicultural rights with a base system of liberalism, then Barry's response is that the result conflicts with and destroys the liberal essence that it is meant to preserve. His general position provides an interesting counterpoint to difference-based liberalism, as does his denial that liberalism is culturally biased, and that equality necessitates a culturally sensitive approach. He also sets out, in a compelling way, how liberalism should treat illiberal minorities.

The core of liberal systems, Barry insists, is a conception of citizenship that admits one status of citizen. ${ }^{81}$ All societies feature difference and conflict, but this conception is best suited to minimising the effect of conflict, and to treating citizens fairly ${ }^{82}$ Such an approach does not ignore or marginalise culture, but, rather, treats it as one aspect of difference that individual liberal rights are best at dealing with. To work towards the promotion and retention of one's minority culture is, he argues, an entirely legitimate purpose, provided that it is achieved without breaching the rights of others. ${ }^{83}$ This purpose is not a legitimate end of political theory in itself, rather, it is the object which an individual or group of individuals pursue.

\footnotetext{
${ }^{80}$ B M Barry, Culture and Equality: an egalitarian critique of multiculturalism (2001).

${ }^{81}$ Ibid, p7.

${ }^{82}$ Ibid, pp19, 21.

${ }^{83}$ Ibid, p66.
} 
In this way, Barry encapsulates what Kymlicka calls the 'benign neglect' approach of liberalism. He asserts that liberalism does recognise fully the value of association, but that this does not imply the need for group rights:

It is true that liberal individualism has the implication that a group has no value over and above its value to its members... but this is quite compatible with a full recognition of the role played in our well-being by the communities and associations to which we belong. ${ }^{84}$

To Barry, then, group and cultural affiliations are appropriately dealt with by a difference-blind form of liberalism that champions the individual right to belong to a group, rather than a group's right to exist and to be promoted to individuals. This approach implies a different relationship between the liberal state and illiberal group than is anticipated by Kymlicka. Surprisingly enough, Barry allows that minority groups can be illiberal:

It is not part of liberalism, as I understand it, to insist that every group must conform to liberal principles in its internal structure. It is a belief that liberalism is committed to this procrustean approach to groups that gives rise to the widely held view that we need some sort of escapeclause to accommodate diversity.... Liberal principles themselves demand that groups should have the utmost freedom to handle their affairs in accordance with the wishes of their members. ${ }^{85}$

This illiberalism in national minorities, and in any social groups, is permissible in this difference-blind form of liberalism, so long as its members consent to it. Genuine consent depends on the availability of low-cost exit options. ${ }^{86}$

In general, this argument holds together much more robustly than that of Kymlicka and the instrumental value of culture to autonomy. A difference-blind liberalism that is based on consent and individual rights could readily permit group influence over individuals provided they consent. It is easy to imagine this rule applying to institutional associations that are clearly voluntarily accepted, such as the joining of a political party or a service association. It is another thing, however, to apply it to cultural groups. Firstly, the choice to accept or reject a culture has an extremely broad effect, potentially, and may include, not only the engagement in certain practices, rituals and institutions, but, also, effective familial membership, geographical location, and, even, moral beliefs. It is hard to justify illiberal restrictions based on consent to a much broader package of items.

\footnotetext{
${ }^{84}$ Ibid., p123.

${ }^{85}$ Ibid, pp147-148.

${ }^{86}$ Ibid, p149.
} 
In addition, even accepting that such a group is a minority and that there is a freedom to integrate into the majority population, as Kymlicka points out, culture is of foundational importance to choice. In other words, the consent on which Barry relies may be in some significant way the product of the culture that is being consented to. Even this position is not sufficiently sophisticated to accurately portray the nature of belonging to a minority culture. Intracultural diversity, intercultural intermingling, and the possibility of internal dissent, all challenge Kymlicka's picture of a minority culture. This does not mean a reversion to Barry's implicit conception of voluntary cultural attachment, as Kymlicka's analysis is not without basis; but, it means that neither Barry's nor Kymlicka's conception is sufficient as a foundation on which to fully explicate personal attachment to culture. In relation to minority cultures, as attractive and achievable as Barry's difference-blind position appears, it seems to be undermined by the underlying fiction of cultural consent.

To Barry's credit he accepts the difficulty of his position, and he frames the consent basis to culture to mean that

\footnotetext{
if you remain in some association that you have the power to leave, that establishes a presumption that the perceived benefits of staying are greater than the benefits of the most attractive alternative.... If... your staying means that you are passing up at least one reasonable eligible alternative, that is a much sounder basis for inferring that the association is not treating you so badly. ${ }^{87}$
}

What Barry seems to ultimately claim is that the right of free association means that members of minority cultures are able to choose, either, to avail themselves of that culture, or, to exit, and integrate into the majority culture. This freedom, which is afforded to all citizens, is sufficient to ensure both that a valuable culture can survive and that a disliked culture will be forced to adapt or be destroyed. This 'democratic' approach to cultural adaptation and survival is attractive in that a culture depends essentially on the will of its members, and also because, unlike that of Kymlicka, can incorporate intermingling and dissent. However, even this weak presumption that members are not being treated so badly does not answer the suggestion that cultural consent is not morally meaningful in the same way that consent of the membership of a particular social association or institution is.

\footnotetext{
${ }^{87}$ Ibid, p149.
} 


\subsection{Liberal bias and equal treatment}

Kymlicka's second argument in favour of multicultural rights rests on the idea that liberalism is biased in favour of majority cultures, and that equal treatment requires that this bias is acknowledged and corrected for. Barry opposes this idea completely, and argues, instead, that some such bias is necessarily a part of any political or moral theory:

The lesson commonly drawn by multiculturalists is that 'liberalism can't and shouldn't claim complete cultural neutrality'. This is a lazy response because no attempt is made to specify what 'complete cultural neutrality' might look like or explain why it should ever have been supposed that liberalism is committed to it. The evidence against liberalism having this property appears to consists in nothing except its incompatibility with some other (obviously incompatible) beliefs.... It would seem that for liberalism - or any other doctrine for that matter - to be culturally neutral, there would have to be no existing... world view with which it conflicts. Since this is manifestly absurd, the assertion that liberalism is not culturally neutral asserts something that could not conceivably be denied. ${ }^{88}$

The value of liberalism, Barry continues, is not that it is neutral in this way, but, rather, that it is fair: that it recognises significant social groups; that it is even-handed in their treatment; and, that it offers the same general rights of association to all members of a society. ${ }^{89}$

In this passage, Barry invokes Taylor's claim that 'liberalism can't and shouldn't claim complete cultural neutrality". The point that Taylor makes is that, contra the difference-blind approach, liberalism is not compatible with all cultures, but, rather, expresses one range of cultures. The example he offers concerns how cultures that do not accept a separation between church and state would be excluded from or marginalized by typical western liberal democracies. ${ }^{90}$ Elsewhere he argues that difference-blind liberalism can force people into a mould that reflects the hegemonic culture, and wonders if expressions of difference-blind liberalism are just "a particularism masquerading as the universal". ${ }^{91}$

\footnotetext{
${ }^{88}$ Ibid, p27. The internal quote is from Taylor, op cit, above note 5, p62.

${ }^{89}$ Ibid, pp28-29.

${ }^{90}$ Taylor, op cit, above note 5, p62.

${ }^{91}$ Above, note 11; Ibid, p43.
} 
Barry replies that it is no surprise that a positive political theory conflicts with some particular beliefs, and that liberalism is the best of such theories in that it is fair and even-handed towards any such belief. This appears to deflate Taylor's accusation of liberalism, that it is cannot be completely neutral: clearly it will be contrary to those beliefs that are illiberal. Difference-blind liberalism would still permit these beliefs to be promoted as part of a minority culture, so long as they are consented to by its members. This appears to be an effective defence by Barry to counter Taylor's assertion about the beliefs liberalism excludes. The latter's argument, as to the cultural mould that is implied in theoretical liberalism, seems closer to the mark in that it suggests that liberalism applies an arbitrary standard, and puts a cultural perspective on communitarian arguments about implicit conceptions of the person. However, Taylor does not present the full reasoning behind this argument.

Kymlicka takes a different approach to asserting the cultural bias of difference-blind liberalism in that he describes the particular political mechanisms by which a majority necessarily imposes its culture on a minority. These, he categorises into three groups: decisions on official languages, political boundaries and the division of powers. The decision as to whether public education will be offered in a minority language, for example, will be important to the continuation of that language, and to the practice of that culture as a whole. ${ }^{92} \mathrm{He}$ asserts similar claims about the drawing of political boundaries within a state, and the distribution of legislative power.

The whole idea of 'benign neglect' is incoherent, and reflects a shallow understanding of the relationship between nations and states. In the areas of official languages, political boundaries, and the division of powers, there is no way to avoid supporting this or that societal culture, or deciding which groups will form a majority in political units that control culture-affecting decisions regarding language, education and immigration. ${ }^{93}$

The crux of Kymlicka's argument is not that difference-blind liberalism favours some practices over others, or in other words, liberal practices over the illiberal. Rather, it is that many fundamental political decisions necessarily favour one culture over another in a way that is completely arbitrary when viewed from a liberal perspective. In other words, difference-blind liberalism is not really difference-blind - decisions that appear to treat each person on an individual basis are in fact loaded with cultural content, and this cannot be any other way. The kind of cultural neutrality that

\footnotetext{
${ }^{92}$ Kymlicka, op cit, above note 24, p111.

${ }^{93}$ Ibid, p113.
} 
liberalism cannot achieve, then, is not the complete neutrality between cultures, but a neutrality already informed by liberal conceptions and contours.

Barry does not deal with these arguments directly. He does, however, address minority language availability, and seems to conclude that public education should normally be carried out in the majority language, as learning the minority language alone may disadvantage a person in their ability to fit into mainstream life, and learning it as a second language would mean giving up the opportunity to learn a potentially more valuable foreign language. ${ }^{94}$

The difference between these approaches seems to rest on an attitude towards and value of culture, and its relationship to language. Kymlicka links the retention of the minority language to retention of the minority culture, and argues that equal treatment may require the promotion of the language through public education. Barry takes a less reverential view of the minority language and culture, and argues that promoting the former may exclude some from access to the mainstream culture. Applying his earlier logic, if individuals do choose to learn the language, then they are free to do so, and this indicates its benefit to them; but if not, then the state should not promote it, and individuals should be free to exit the minority culture and integrate into the majority.

The problem with this argument is that Barry treats the majority culture as some kind of default, by virtue of is central political and economic presence. In effect, Barry is not really denying the de facto cultural bias of liberalism, rather, he is justifying it in favour of the majority culture, because that culture is already central to the state. Ultimately, his arguments depend upon the legitimacy of leaving an individual to decide that being attached to a minority culture is too much of a disadvantage relative to the benefits of being able to engage completely in the political and economic mainstream. This, he would argue, is not really a bias, but, rather, a social and practical reality refracted by individual consent.

\footnotetext{
${ }^{94}$ Barry, op cit, above note 80, pp105-108.
} 
Kymlicka would respond that this and other political decisions do not treat the minority culture fairly, as they ignore its claims to occupy some of this political centrality. Ultimately, the liberal expression of the goals of these minority cultures is to undermine this view of the majority culture as the default, and to assert themselves as viable and continuing. This does not mean that they aspire to take over from the majority culture, or to compete with it as an alternative, but, rather, they aspire to have a real and viable presence within the society as a whole, however this manifests, and to not be excluded from achieving this simply because the majority is the easier and most practical subject of political decisions. In this way, Kymlicka's assertion as to the political cultural bias of liberal states is well-founded. This does not necessary mean that the full compliment of group rights should be available to every minority, but it does at least provide a positive theoretical basis for a culture-sensitive liberalism.

The final criticism of multiculturalism from Culture and Equality to be examined here relates to the interaction between a minority culture and general society. This point is important to multicultural liberalism as a practical political approach, but, here, can be considered on more principled terms. Barry makes the general point that identifying some practice or behaviour as part of one's culture does not amount to a sufficient justification. Various cultures contain strands that are extremely harmful to others. ${ }^{95}$ Culture should not be the excuse for such actions.

If there are sound reasons against doing something, these cannot be trumped by saying - even if it is true - that doing it is a part of your culture. The fact that you (or your ancestors) have been doing something for a long time does nothing in itself to justify your continuing to do it. $^{96}$

A difficult part of multicultural liberalism is that the promotion of culture can include the protection of particular cultural practices that are harmful or destructive. One form of these are the internal restrictions already discussed. But, the focus here is more on particular patterns of behaviour that are harmful to those who are not adherents to that culture; in other words, where there is conflict between liberalism and culture, or at a social level, between minority and majority cultures. Barry would admit individuals the freedom to pursue whatever conception of the good they choose, which would

\footnotetext{
${ }^{95}$ Ibid, pp253-254.

${ }^{96}$ Ibid, p258.
} 
include allowing cultural practices. If such practices breach the freedoms of others, however, he would argue that liberal principles should prevail, as "[c]ompromise over liberal principles is not, and cannot be, a liberal value". ${ }^{97}$

The general approach taken by Kymlicka and other multicultural liberals is that neither majority values nor minority practices will automatically prevail, but that a contextual examination of the issue should occur. This typically involves the weighing up of the proposed activity, and, in particular, its cultural importance, along with the significance of the imposition that this activity places on others.

\subsection{Liberalism and multiculturalism}

Exploring the work of Taylor and Kymlicka offers a basis for a multicultural form of liberalism. Such a liberalism would allow group rights as exceptions to the normal difference-blind approach, and, so, would be culture-sensitive in such a way as not to destroy the underlying liberal structure. Three bases can be discerned for this from the two theorists. Taylor's focus is on the harm of misrecognition that applies to culturally informed identities, and corrects for the cultural assumptions that underlie any particular liberal conception. Kymlicka's first justification of group rights depends on the instrumental importance of culture to the exercise of autonomy, but is not adequately explained and suffers both from a monolithic definition of culture and difficulties in relying on autonomy as the source of cultural value.

The most fully explained argument, and best candidate as a source for group rights, is Kymlicka's suggestion that equality requires culturally-differential treatment. This depends on the assertion that difference-blind liberalism is biased in favour of the majority culture in a significant and morally arbitrary way. This turns out to be a difficult task, as the attractions of Barry's conception of a liberalism based on individual-rights are considerable. They suggest that it is best to treat the majority culture as the standard, and to leave the minority culture to flourish or to die on its own virtues. This ignores, however, that these virtues will always be subject to the

\footnotetext{
${ }^{97}$ Ibid, p283.
} 
majority culture as the baseline for social interaction. This is not to say that there is no practical or moral value to having a majority culture, or that the mere fact of one implies injustice, but, rather, that minority cultures face a system that works from assumptions that can amount to significant and morally arbitrary barriers to its continuation, and that disadvantage its members.

Barry's general approach is based on a model of culture as choice: that each member of a cultural minority is free to either adhere to or reject their culture. This means that the value of culture is entirely internalised and, so, expressed by the freedom of association, which is uniformly recognised. This approach does not seem to capture the relationship between individuals and culture, its weight, meaning and the nature of the attachment.

This should not take away from the important practical concerns that are raised by Barry in Culture and Equality. Barry identifies that there are real and potentially substantial costs to deviations from the difference-blind approach. There is the potential for group rights to protect conservative and illegitimate elements of minority cultures, and generally to reduce a singular conception of citizenship to a politics of culture. On theoretical grounds, however, there is a valid basis for a liberalism that is sensitive to its own cultural effects. In examining Kymlicka's framework for group rights, there are two persistent concerns central to any similar form of multicultural liberalism. They relate, firstly, to the conception of culture that powers the theory, and, secondly, the account of liberal cultural bias that justifies culture-specific rights.

The works of Taylor, Kymlicka and even Barry seem to depend on grossly simplified conceptions of culture. This is most obvious for Kymlicka, where a totalistic approach to culture, specifically in regard to national minorities, is employed. His account ignores the diversity present within a culture, including cultural disagreement and dissent; and the cultural intermingling between minority and majority culture, and between different minority cultures. In addition, all of the theorists gloss over other less obvious cultural complexities, such as the relationship between a particular culture and its adherents, which describes the person's cultural attachment. Also included are the methods by which a culture sustains itself, the power relationships internal to a culture, and the culture's attitude to and method of self-transformation. 
Each of these is potentially relevant to how a liberal theory should incorporate culture, and how a state should treat particular cultures, but Kymlicka addresses none of these. ${ }^{98} \mathrm{He}$ is no doubt aware of these difficulties, but is interested in starting from straightforward yet common multicultural situations. This is undermined, since his conception of culture is simplified to the point where the theory itself seems practically unusable.

It is a necessary part of a project of multicultural liberalism that common patterns of culture and cultural situation are applied in order to be able to theorise in a meaningful way. It is also important, however, that the theory be sufficiently representative of the diversities that are present in social reality; the whole point of this area is to recognise that an accepted mode of thought may hide cultural bias and, so, may make an assumption of singularity that is not justified in the theory itself. It seems, then, that multicultural liberalism requires a more complex conception of culture than is used by Kymlicka. Such a conception would play a key role in elucidating cultural difference and, in particular, in distinguishing between intracultural pluralism and cultural pluralism, so as to explicate liberalism's potentially differential response to both.

The second issue raised by Kymlicka's multiculturalism relates to the cultural bias he identifies in liberal theory. His general approach is to assert the importance of group rights that are new to liberal theory, in order to respond to concerns that are similarly new. As such, his identification of cultural bias concerns political decisions that are not normally the concerns of liberal theory: public languages, political boundaries and the division of powers. In this way, Kymlicka's group rights are able to attach relatively easily to a base liberal theory without undermining that theory generally. However, it is possible that, if liberal bias applies to these political decisions, then it also applies to other more obviously liberal issues. This result, however, would bring the entire scheme of liberal theory into question.

In the first three substantive chapters, one of the primary criticisms of liberal theory focused on a form of bias: that the various approaches did not by themselves adequately justify their particular substantive prescriptions, and that, instead, many of

\footnotetext{
${ }^{98}$ With the exception of noting and finding irrelevant the internal political and social realities.
} 
the points made by the theorists remain the subject of rational contestability. This led to interpretations, conceptions and structures that appear plausible, but that, in fact, reflect the personal approach of particular theorists. Multicultural liberalism raises the possibility that there could be a cultural aspect to this bias, so that these systems reflect particular cultural views, or that the substantive principles are unfair to some cultures.

This issue has led to liberal theories that are founded on difference, be it some form of pluralism or reasonable disagreement, and that employ less strident justificatory strategies. In particular, contextual approaches have been employed by various theories in order to achieve some form of justification. In this way, autonomy, pluralism, reasonableness, and even adherence to liberalism itself have been suggested as effective limits on liberal justification. ${ }^{99}$ These assumptions may be important to building a relatively coherent liberal system, but, as this exploration of multiculturalism has shown, the level of intra and intercultural diversity makes identifying society-wide social patterns very difficult. This is especially the case in relation to a value that is supposed to be paramount, such as autonomy. This is not to say that contextual approaches are not possible, but that, when they specify contingent social patterns, they need to be aware of the relevance of cultural pluralism.

Overall, if multicultural liberalism is to be based on a view of liberal cultural bias, then this view must be fully explored, and its limits must fit with the structure of the liberal content of the theory. What is disconcerting about Kymlicka's approach is that he limits cultural bias to just a few political decisions, all of which are not significant to normal liberal theories, and he never addresses whether this bias extends to the more basic liberal ideals. This is understandable, since a positive result would undermine Kymlicka's liberal structure, one that is augmented by a few group rights.

\section{Multiculturalism beyond liberalism}

\subsection{Demands for recognition}

\footnotetext{
${ }^{99}$ E.g. Raz, Gray, Rawls and Waldron respectively.
} 
The remainder of this chapter will examine approaches to multiculturalism that respond to these two issues, and employ conceptions of culture and bias that are more developed than those used by Kymlicka. The first of these concerns James Tully's book Strange Multiplicity: Constitutionalism in an age of diversity (1995). ${ }^{100}$ In many ways, his project is an extension of that of Taylor, and his basic aim is to sketch out an approach that can deal with the "multiplicity of demands for recognition at the same time". ${ }^{101}$

Tully's approach is not purely one of liberalism, and, instead, is based on a broader view of constitutionalism that incorporates, at the very least, liberalism, nationalism and communitarianism. A complete political approach is sensitive not only to cultural diversity, Taylor argues, but also to the diversity of traditions within political theory. It is important, then, for each of these three traditions to be part of a constitutional dialogue; any approach short of this would be poorer for it. ${ }^{102}$ As such, Tully argues that Kymlicka's work is valuable, but that it fails to question the value of liberalism as the 'sovereign' political tradition. ${ }^{103}$ Despite these doubts as to the overarching value of liberal theory, Tully's work is interesting in that it is at least partially bound to the liberal viewpoint, and in that it presents an extended conception of culture and cultural diversity, and of bias, and derives from them a positive substantive approach to cultural recognition.

Tully asserts that the 'billiard-ball' view of culture, as being bounded, separate and internally uniform, has given way to a more detailed and more complex conception, driven in part by actual demands for recognition.

\footnotetext{
processes of interaction with other cultures. The modern age is intercultural rather than massive migrations of this century. Cultural diversity is not a phenomenon of exotic and are members of more than one dynamic culture and the experience of 'crossing' cultures is

${ }^{100}$ J Tully, Strange Multiplicity: Constitutionalism in an age of diversity (1995).

101 Ibid, p6.

102 Ibid, p57.

${ }^{103}$ Ibid, p53.
}

Not only do the cultures overlap geographically and come in a variety of types. Cultures are also densely interdependent in their formation and identity. They exist in complex historical multicultural. The interaction and entanglement of cultures has been further heightened by the incommensurable others in distant lands and at different stages of historical development, as the old conception of culture made it appear. No. It is here and now in every society. Citizens normal activity.... Finally cultures are not internally homogeneous. They are continuously 
contested, imagined and reimagined, transformed and negotiated, both by their members and through their interactions with others.... Cultural diversity is a tangled labyrinth of intertwining cultural differences and similarities, not a panopticon of fixed, independent and incommensurable worldviews in which we are either prisoners or cosmopolitan spectators in the central tower. ${ }^{104}$

For Tully, this means that cultures are overlapping, interactive and internally negotiated. The basic ideas that are encompassed by these points emphasise that: contemporary culture does not exist on its own, but, rather, is in a permanent state of interaction with other cultures, such that a culture's meaning is, at least partially, expressed by those relationships; people can belong to multiple cultures, and, by inference, people can relate and attach differently to particular cultures; and finally, cultures are themselves diverse and are continually being reinterpreted.

Tully also identifies that a consequence of this conception is that "the experience of cultural difference is internal to culture". ${ }^{105}$ Instead of having a fixed cultural identity, and treating those outside of this as the other, our cultural identities are shifting such that otherness is internal to our cultures and ourselves. Tully grants that this point is difficult; and, he refers to postmodern philosopher Jacques Derrida's idea that a culture should not be identical to itself, meaning that it should not be able to identify itself. $^{106}$

This statement is difficult, and does more than just recognise that a culture is or should be diverse. Rather, it seems to assert that a contemporary culture, by virtue of the overlap and interaction between cultures, and its internal diversity, cannot operate as a totality, and, instead, internalises a sense of self-limitation and a necessary reference to the other. In other words, the nature of culture incorporates cultural difference. This result is important for Tully because it means that the intercultural dialogue that he prescribes is rendered possible by virtue of the fact that each person's identity and self understanding already incorporates a form of cultural dialogue.

Next, Tully explores the history of constitutional thought. He examines in detail many of the sources of this constitutionalism, and finds that the dominant thread presents an

\footnotetext{
${ }^{104}$ Ibid, pp10-11.

105 Ibid, p13.

${ }^{106}$ Ibid, pp13-14.
} 
approach that "was designed to exclude or assimilate cultural diversity and justify uniformity". ${ }^{107}$ This is manifested in the language that is used by constitutionalism.

[T] he language of modern constitutionalism that has been forged in constitutional theory and practice over that last three hundred years is a partial forgery. While masquerading as universal it is imperial in three respects: in serving to justify European imperialism, imperial rule of former colonies over Indigenous peoples, and cultural imperialism over the diverse citizens of contemporary societies. When members of the authoritative schools today write about constitutionalism, whether they claim to be universal, historical or transcendental, they do so within the conventions of universality, history and transcendence of this captivating map of mankind. They (and this often includes myself) think that they are tracing the contours of humanity's constitutions, yet they are merely tracing round the 'splendorous' frame through which they look at them. ${ }^{108}$

Tully posits that constitutionalism has suffered from the error of uniformity, and, so, is effectively biased against cultural diversity. It is from the perspective of this uniformity that modern society appears a 'strange multiplicity'. ${ }^{109}$ Liberalism, being one contemporary tradition of constitutionalism, suffers also from this bias, and it is certainly possible to see, in the work of Rawls, Nozick and Dworkin, the perspective of universality that Tully registers. This view of bias is much broader than that of Kymlicka, in that it applies not to a few political decisions, but rather to the nature of the way in which political decisions generally are thought about and made. It paints a picture of liberal theory being caught up in uniformity and universality that is an expression of the imperial modes of western thought and culture.

It is interesting that Tully makes the link between imperialism and uniformity, in the liberal sense of difference-blindness. It is a natural enough step to take, since such imperialism implies asserting authority over and integrating foreign populations. The difference-blindness of liberalism, however, is sincerely believed by its adherents to be a tool that empowers and offers liberty to individual members of a society, rather than coercing them into cultural agreement; and that this form of liberalism champions the right to decide how one should live. It is beyond the scope of this thesis to remark on this historical truth and the significance of Tully's account of constitutionalism, and its ultimate influence on liberalism, but it does offer an explanation of the failings of early contemporary liberalism: it works from the

\footnotetext{
${ }^{107}$ Ibid, p580

${ }^{108}$ Ibid, p96.

${ }^{109}$ Ibid, p58.
} 
perspective of universally correct liberal principles; and, it accepts what could be described as the myth of the fairness of uniformity.

Without an examination of Tully's historical account of constitutionalism, it is difficult to accept fully his conclusions. From a theoretical perspective, however, Kymlicka's work shows how uniformity in liberal approach can disadvantage minority cultures. Tully's assertion that this is potentially endemic to liberalism makes sense in that Kymlicka's account of this bias seems unduly restricted. In addition, it is certainly the case that earlier contemporary liberal theories did not achieve the universality to which they aspired.

If this assertion of general bias is valid, then liberalism is left in a difficult position, in that it undermines the application of uniform rights. It effectively brings into sharp focus the tension between culture and the aspirations of liberalism to provide a culture-indifferent and yet fair system of political and social organisation. In defence of liberalism, Tully's account is most germane to those earlier forms that have already been the subject of similar communitarian criticisms. Later developments in liberal theory have set difference as the central liberal concern. Whether they are able to incorporate or, otherwise, deal with cultural difference is the challenge that Tully's account of constitutionalism sets.

Because Strange Multiplicity is ultimately a positive theory, Tully himself must deal with cultural diversity. His approach is avowedly constitutional rather than liberal, yet it contains some points relevant to a culturally-sensitive liberalism. The political goal of Tully's work is to achieve a fair dialogue between different cultures.

A constitution should be seen as a form of activity, an intercultural dialogue in which the culturally diverse sovereign citizens of contemporary societies negotiate agreements on their forms of association over time in accordance with the three conventions of mutual recognition, consent and cultural continuity. ${ }^{110}$

Much like liberal theories of public reason, Tully focuses on the value of a dialogue in mediating cultural diversity. As with those approaches, conditions of 'reasonableness' are required as the basis from which to interact fairly with one another. The three conditions of mutual recognition, consent and cultural continuity, combined with the

${ }^{110}$ Ibid, p30; see also p184. 
further idea of cultural respect, ensure that all parties engage in these dialogues with an attitude that promotes cultural sensitivity. ${ }^{111}$

The resulting process aims not at consensus or some universal goal, as "the world of constitutionalism is not a universe, but a multiverse". ${ }^{112}$ Despite this, the result is just because it incorporates the three traditions of constitutionalism on one hand, and cultural diversity on the other. He explains this in relation to the distinction between just and mediated peace:

A just peace is a constitutional settlement in accordance with the comprehensive theory of justice.... [T] his is the most destructive illusion of our age. The just peace of the theorists and dogmatists is neither justice nor peace. It is always a limited and partial description of justice which, when imposed, galls the necks of others and leads to discords. On the contrary, a mediated peace is a just peace: just because it is a constitutional settlement in accord with the three conventions of justice and peaceful because the constitution is accommodated to the diverse necks of those who agree with it. ${ }^{113}$

The proper nature of Tully's justice, then, is that it incorporates a balanced dialogue between the traditions of political theory, and between the cultures that inhabit a particular society. It is because these sets of beliefs have developed and survived that respecting them is just. Their value rests in the adherence of individuals who, by virtue of their place in the modern world, have some capacity for shifting their cultural identity. It is significant, then, that Tully's position is not culturally conservative: within each individual, and the system of dialogue that implies diversity, is the power for social criticism and dissent. ${ }^{114}$

Tully strengthens this conception of justice with a discussion of the intrinsic value of diversity. He quotes favourably from essayist Octavio Paz:

What sets worlds in motion is the interplay of differences, their attractions and repulsions. Life is plurality, death is uniformity. By suppressing differences and peculiarities, by eliminating different civilizations and cultures, progress weakens life and favours death. The ideal of a single civilization for everyone, implicit in the cult of progress of technique, impoverishes and mutilates us. Every view of the world that becomes extinct, every culture that disappears, diminishes a possibility. ${ }^{115}$

\footnotetext{
${ }^{111}$ Ibid, p190.

112 Ibid, p131.

113 Ibid, p211.

114 Ibid, p207.

${ }^{115}$ Ibid, p186.
} 
This is an interesting view of diversity, and goes further than any of the liberal accounts already discussed. It suggests that all world views are inherently valuable on the basis of their difference from other views. This paints rather an extreme picture of diversity that does not accord with the rest of Tully's theory. Paz's antipathy to the extinction of cultures, for example, does not quite fit with Tully's belief in dissent and cultural transformation. Tully seems much more comfortable with the idea that a culture, or an old form of a culture, might give way to the new, and that this process is part of healthy social criticism. Also, Paz's view implies that the ideal is to maximise diversity, so that there is the greatest possible number of cultures, each with different belief systems, values and languages. In contrast, Tully's view is that there should be a dialogue between those cultures and traditions that do exist.

Despite the positive way in which Tully quotes Paz, it seems that their views of diversity are quite different: Tully wants to argue in favour of the diversity of those cultures and traditions that are already adhered to, whereas for Paz, the value is in the diversity itself. Ultimately, Tully's justice is based on respecting the adherence to a set of beliefs, more than the diversity of including as many views as possible in a dialogue. He is concerned with the necessity of a dialogue between those systems that enjoy significant adherence.

\subsection{Cultural understandings of culture}

Strange Multiplicity is interesting because it offers a more developed account of culture, which is then used to support a theory of multicultural justice. The basis of this theory is that it respects cultures by prescribing that they engage in a fair dialogue. This empowerment of cultures is informed by the innate capacity for cultural criticism and dissent. Because this is not simply a liberal theory, it is not necessary to examine this prescriptive structure in close detail, but Tully's account of culture and its role within the theory remains important.

Colin Perrin has criticised Tully's account of culture for being so concerned with the cultural multiplicity to which each person is subject that he loses connection with any 
meaningful conception of cultural attachment: when intracultural diversity and dissent, and intercultural dialogue are the central ideas of culture, there is the risk of forgetting his original point, that individuals and groups demand cultural recognition. ${ }^{116}$ It is a valid point that Tully should not lose sight of the value and meaning of cultural attachment, especially relating to groups that resemble Kymlicka's national minorities.

A more general and more rigorous commentary on Tully's conception of culture has been undertaken by David Scott. He notes that Tully historicises the ideas and language of political philosophy, and attempts to look beyond the historically contingent tendency to universal, uniform and assimilationist thought. Scott argues that Tully's approach to culture is quite different, and is naïve and blindly accepting by comparison. ${ }^{117}$

\begin{abstract}
Where in the old days... political philosophy depended upon a false... conception of culture, now thanks to recent hard-won developments in anthropology there is a new and correct conception that will at once free political philosophy of its prejudices and enable it to more adequately reformulate the problem of constitutionalism. This story has about it a curiously just-so character. ${ }^{118}$
\end{abstract}

Scott asserts that culture is potentially as historically contingent as constitutionalism.

He goes on to sketch the possible development of culture, and, in particular, identifies it as the concept by which western peoples distinguish themselves from the nonEuropean other. ${ }^{119}$ This concept developed to replace alternative now-defunct points of distinction, such as the absence of reason, and race. Culture amounts to the twentieth century's account of otherness. ${ }^{120}$

Scott suggests that the understanding from which Tully borrows is of culture as constructed meaning, that it is, by nature, antifoundational and is accessible only from an interpretive framework. ${ }^{121}$ Cultures, in terms of this view, are not tethered to a particular world view or understanding; rather, they are created dynamically by group

\footnotetext{
${ }^{116}$ C Perrin, Book review: Strange Multiplicity: Constitutionalism in an Age of Diversity by James Tully (1999) 10(2) European Journal of International Law 468, p470.

${ }^{117}$ D Scott, 'Culture in Political Theory' (2003) 31(1) Political Theory 92, p102.

${ }^{118}$ Ibid, p101.

${ }^{119}$ Ibid, pp103-104.

${ }^{120}$ Ibid, p104.

${ }^{121}$ Ibid, pp107-108.
} 
behaviour, and are understood as such, only from the cultural perspective of the spectator.

Scott makes these claims not so as to refute Tully's conception of culture, but, rather, to insist that this perspective should be reflected in his theory, just as the historicism of constitutionalism is reflected. ${ }^{122}$ For Strange Multiplicity, and for multicultural liberalism generally, Scott's suggestion is disconcerting. It means that culture is the contingent output of an evolution of ideas, and, so, even if rendered complexly will always be open to change and to rational contest. This does not deny culture any meaningful content, but does at least make its content variable and uncertain.

One possible response to Scott's argument would be to say that all theoretical conceptions are the result of a historical evolution. This does not, in itself, make them wrong, and the fact of development in a particular direction actually suggests the value of the result, perhaps not as the final word on the subject, but at least as the most appropriate prima facie approach to take. This argument is undermined by the diversity of ideas: the fact that contestability, in a living theoretical area, will usually mean contested, so that a single result is not always available.

In relation to Tully's general scheme, the overriding value is that of adherence: that a tradition or culture that is commonly adhered to is deserving of consideration and expression within a political intercultural dialogue. His view of liberalism is as a tradition that deserves considerable political focus because of its popularity. This is an interesting account, but one that fails to ask why liberalism is adhered to in this way, and, so, to relate a conception of tradition to moral and theoretical value.

\subsection{Rethinking Multiculturalism}

Bikhu Parekh, in his book Rethinking Multiculturalism: Cultural Diversity and Political Theory (2000), attempts to use multiculturalism as the perspective from

${ }^{122}$ Ibid, p106. 
which to move beyond liberalism. ${ }^{123}$ This move takes multiculturalism one step further than does Kymlicka's position, in that Parekh is willing for multicultural insights to trump liberal values. Parekh works, ultimately, from a view of a shared humanity that is mediated by culture. ${ }^{124}$ This view implies the cultural basis of liberalism as a particular doctrine, espousing a particular view of human nature and society. The best response to the fact of cultural pluralism, he argues, is not to create a liberal theory of multiculturalism, as

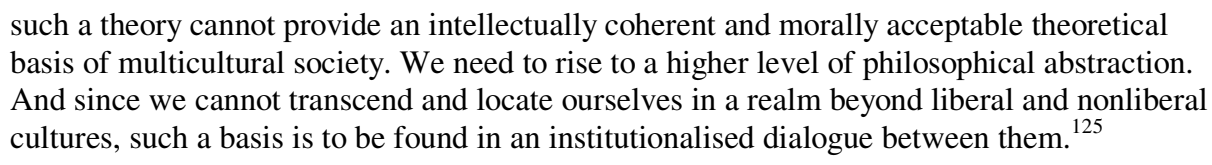
basis of multicultural society. We need to rise to a higher level of philosophical abstraction. And since we cannot transcend and locate ourselves in a realm beyond liberal and nonliberal cultures, such a basis is to be found in an institutionalised dialogue between them. ${ }^{125}$

While liberalism may be a popular doctrine, it is not in itself sufficient for a full theory of societal justice. To go beyond the liberal ideals requires the positive engagement with other, non-liberal cultures, by way of an institutionalised dialogue.

Kymlicka goes on to criticise liberal approaches to multiculturalism. He notes specifically that Kymlicka's work is deficient in not presenting a suitable conception of the value of cultural diversity. ${ }^{126} \mathrm{He}$ also criticises contemporary liberal theorists generally on three grounds: that they are too committed to the transcultural view of people, and fail to recognise fully their cultural embeddedness; that they absolutise liberalism, and subordinate non-liberal ways of life; and, that they generally treat nonliberals badly. ${ }^{127}$

Parekh's approach, in contrast, champions diversity and intercultural dialogue, and is based on the conception of a pluralist universalism. He identifies three layers to our identities, namely our human, cultural and individual natures. ${ }^{128} \mathrm{He}$ argues that, in moral theory, neither monism nor relativism is suitable in acknowledging the complexities of our identities, as monism ignores the role of culture in mediating our

\footnotetext{
${ }^{123}$ B C Parekh, Rethinking Multiculturalism: Cultural Diversity and Political Theory (2000).

124 Ibid, p11.

${ }^{125}$ Ibid, p14.

${ }^{126}$ Ibid, p108.

${ }^{127}$ Ibid, pp109-111.

${ }^{128}$ Ibid, p123.
} 
shared humanity, and relativism ignores the shared humanity that underlies our constructed cultures. ${ }^{129}$

For Parekh, the approach that makes the most sense is a minimum universalism, which reflects the dual roles of humanity and culture. Such an approach tends to ignore the conflict between minimum universal values. So, the best form of this universalism is one that incorporates a pluralist and dialectical viewpoint; in other words, one that accepts a plurality of expressions of universal values, and employs a dialogue to deal with and overcome this. ${ }^{130}$

Since we are culturally embedded and prone to universalizing our own values, we need the dialogue to counter this tendency and help us rise to the required level of intellectual abstraction. The dialogue also brings together different historical perspectives experiences and cultural sensibilities, and ensures that we appreciate human beings in all their richness and that the values we arrive at are as genuinely universal as is humanly possible. ${ }^{131}$

As with Tully, Parekh uses dialogue as the central mechanism by which cultural pluralism is accommodated. For Tully, dialogue is the means for respecting fairly the popular cultures and traditions, and, so, achieving a richer and more valuable result than any one culture could offer. Parekh is more obviously focussed on the power of dialogue, not to meld different beliefs, but to attain a moral result that is as universal as possible. In this way, his is the clearest adoption of Habermas' ideas of dialogical morality.

It is important to consider exactly what Parekh understands culture to mean. He begins in much the same way as Kymlicka, by describing the embeddedness of individuals in their cultural beliefs, and how the latter offer both context and meaning to individual action.

Culture is a historically created system of meaning and significance or, what comes to the same thing, a system of beliefs and practices in terms of which a group of human beings understand, regulate and structure their individual and collective lives. It is a way of both understanding and organizing human life. ${ }^{132}$

As would be expected, Parekh develops this conception of culture further, to go beyond Kymlicka's idea of societal culture. He emphasises, firstly, that cultural

\footnotetext{
${ }^{129}$ Ibid, p127.

${ }^{130}$ Ibid.

${ }^{131}$ Ibid, p128.

${ }^{132}$ Ibid, p143.
} 
belonging is not homogeneous, and, secondly, that cultural identity is "complex and diffused, [and] cannot be summed up in a neat set of propositions". ${ }^{133}$ Both this complexity and internal variation are essential to an understanding of culture.

Because of the complexity of culture, every citizen must practice selectivity in determining which aspects to adopt. ${ }^{134}$ From this point, Parekh goes on to describe the cultural nomad, who owes loyalty to no particular culture but picks and chooses between beliefs and practices. Parekh warns that, although such an approach can be innovative, there is the risk that the nomad loses their self-identity and social understanding. ${ }^{135}$ Parekh seems to accept here that there is a diversity of approaches to cultural attachment. This idea gives way to a cultural view of cultural attachment, whereby the way in which individuals link to culture is itself mediated by that culture. One particular culture, for example, might encourage a degree of cultural nomadism, while another expects total, unquestioning faith. This is a further complexity that should be acknowledged by a conception of culture.

Next, Parekh notes the interdependence between culture and society: culture both influences and is influenced by the circumstances and institutions of a society. ${ }^{136}$ In particular, the economic and political patterns of society attempt to legitimise themselves through influencing the cultural and moral perspective of citizens. This perspective is typically challenged by the marginalised or oppressed. ${ }^{137}$ In this way, Parekh goes one step further than other theorists, and actually incorporates a culture's internal power structure into his conception of culture, and identifies culture as a tool of oppression.

Finally, Parekh emphasises the importance of cultural interaction. He paints a picture of unavoidable mutual cultural influence that is significant for all cultures, and that is only increasing in the context of globalisation. ${ }^{138} \mathrm{He}$ adds a pointed critique of western cultural influence, arguing that the west's interactions with other cultures "occur under grossly unequal conditions, and those at the receiving end often find it

\footnotetext{
${ }^{133}$ Ibid, pp148-149.

${ }^{134}$ Ibid, p150.

${ }^{135}$ Ibid.

${ }^{136}$ Ibid, pp151-152.

${ }^{137}$ Ibid, p152.

${ }^{138}$ Ibid, p163.
} 
difficult to make autonomous choices". ${ }^{139}$ He also warns against the dangers of the uncritical adoption of western norms.

As with Tully's, this view of culture is certainly more compelling than that of Kymlicka. Both Parekh and Tully offer valuable perspectives on our cultural and multicultural lives. However, they differ in significant ways: for Tully, all people are cultural nomads, adhering to multiple cultures simultaneously. Whoever is correct, Parekh manages, unlike Tully, to hold onto the basic idea of individuals embedded in culture. His overall conception is of the robustness of particular cultures and their hold on individuals; this differs from Tully's conception of the necessary fusion and intermingling of cultures in the lives of each person.

The next part of Parekh's theory concerns the value of diversity, a point on which he considers liberal theory to be deficient. He advances a number of potentially valuable arguments in favour of diversity, including the argument that it is important to the exercise of freedom. His main argument for diversity is expressed in terms of the effect of exposure to other cultures:

\begin{abstract}
Since human capacities and values conflict, every culture realizes a limited range of them and neglects, marginalizes and suppresses others. However rich it might be, no culture embodies all that is valuable in human life and develops the full range of human possibilities. Different cultures thus correct and compliment each other, expand each other's horizon of thought and alert each other to new forms of human fulfilment. The value of other cultures is independent of whether or not they are options for us.... By cherishing such commendable values and sensibilities as harmony with nature, a sense of ecological balance, contentment, innocence and simplicity, which our way of life has to sacrifice in order to obtain its characteristic form of excellence, it both reminds us of our limitations and reassures us that the values are not lost altogether. Its inassimilable otherness challenges us intellectually and morally, stretches out imagination, and compels us to recognize the limits of our categories of thought. ${ }^{140}$
\end{abstract}

So, to Parekh, the value of diversity comes from reminding a culture that there are other, valid perspectives and methods of human flourishing. This point has the effect of expanding one's horizons so-to-speak, offering new ideas and sensibilities; but, it also keeps us culturally self-aware by suggesting the limitations of our own perspectives and beliefs. Quite clearly, then, the relevance of diversity for Parekh, relates to the effect of each culture having access to and experience of others.

\footnotetext{
${ }^{139}$ Ibid, p164.

${ }^{140}$ Ibid, p167.
} 
Much of the remainder of Rethinking Multiculturalism is aimed at building a conception of the properly multicultural society. Such a society, according to Parekh, would be "stable, cohesive, vibrant and at ease with itself". ${ }^{141}$ The basis for this result depends on the closely related features of a shared culture, a national identity and a fair dialogue. Firstly, the shared culture is necessary to the functioning of a society, but, of course, must encompass a plurality of different cultures. This culture "forms the basis of a shared way of life, and underpins and gives the state moral and emotional roots". ${ }^{142}$ It develops from the interaction of the different cultures in dialogue, and encompasses the values of fair multicultural interaction. ${ }^{143}$

This shared culture is underpinned by a national identity, which specifies the selfunderstanding of the society as a political community. This identity assists individuals in accepting themselves as part of the multicultural community. ${ }^{144}$ However, it must be simple and thin in order to prevent it from causing conflict and overwhelming the cultural identities of its members. ${ }^{145} \mathrm{He}$ recognises that the formation of such an identity is not an easy process, but that this can be directed by the values of an open multiculturalism and a respect for minority cultures. ${ }^{146}$

Parekh also discusses in some detail the workings of an effective dialogue. Intercultural dialogue should involve, Parekh argues, recognising the fundamental rights of all who participate, and involve each group accepting others as 'equal conversational partners'. ${ }^{147}$ It succeeds only if "the participants enjoy a broad equality of self-confidence, economic and political power, and access to public space". ${ }^{148}$ These conditions imply the confident attachment of individuals both to their own cultures, and to societal multicultural beliefs. It is the presence of these overlapping attachments, as held by participants in the dialogue that offers the best chance for a mutually positive outcome that reflects Parekh's stable, cohesive and vibrant society.

\footnotetext{
${ }^{141}$ Ibid, p236.

${ }^{142}$ Ibid, p224.

${ }^{143}$ Ibid, pp219-224.

${ }^{144}$ Ibid, p230.

${ }^{145}$ Ibid, p231.

${ }^{146}$ Ibid, pp235-236.

${ }^{147}$ Ibid, pp208, 337.

${ }^{148}$ Ibid, p337.
} 
Finally, Parekh makes a significant contribution to the discussion of multicultural equality. With Kymlicka and Tully, he asserts that equality should not be grounded in uniformity. Equality, Parekh asserts, should be built on the interplay of uniformity and difference that characterises humans. ${ }^{149}$

Equality involves equal freedom or opportunity to be different, and treating human beings equally requires us to take into account both their similarities and differences. When the latter are not relevant, equality entails uniform or identical treatment; when they are, it requires different treatment. ${ }^{150}$

In this way, Parekh deftly retains uniform treatment, except when differences require otherwise. The concern with allowing this differential treatment, he comments, is to ensure that it does not mean discrimination or privilege. ${ }^{151}$ In order to reduce the possibility of this, he sets out a process by which these issues of equal treatment should be dealt with as part of an intercultural dialogue. At the heart of this approach is the idea that, when minority practices offend the public values of the majority, the minority should explore the meaning and value of the practice, and attempt to defend it to the majority. ${ }^{152}$ The majority is then able to disallow the practice should it inhibit, in a relevant sense, their way of life. ${ }^{153}$ Although Parekh's description of this process is quite bare, it does offer a firm and cogent account of the accommodation of minority practices, and is much more developed than that of Kymlicka or Tully.

\subsection{Multicultural attitudes to liberalism}

Parekh's conception of multiculturalism is attractive in that it adopts a realistic conception of culture and champions the power of intercultural dialogue, not merely as a modus vivendi between conflicting groups, but in order to assist society in moving towards universally accepted values and principles. Towards liberalism he displays a quiet appreciation, tempered by a number of substantial criticisms. Like Tully, he views liberalism as a substantive doctrine; but one that has its limitations. ${ }^{154}$ As a theory, liberalism tends to be absolutised, and to marginalise non-liberals.

\footnotetext{
${ }^{149}$ Ibid, p240.

${ }^{150}$ Ibid.

${ }^{151}$ Ibid, p261.

${ }^{152}$ Ibid, pp270-271.

${ }^{153}$ Ibid, p273.

${ }^{154}$ Ibid, p14.
} 
Although he does not explicitly accuse liberal theory of cultural bias, he treats it as a part of western political culture, and implies that its marginalising effect is particularly relevant for non-western minority groups.

In terms of the analysis of earlier chapters, Parekh seems to view liberal theory as operating in a contestable space, and criticises its assertion of universality, and, consequently, its exclusion of those who do not fit within its confines. This seems a reasonable view of the early contemporary liberal theory, one that is conscious of the subordination of non-liberals: that their beliefs are reduced to operation in the private sphere, or within the realm of personal conceptions of the good. These non-liberals are marginalised because, although they have significant freedom within which to determine their own beliefs and follow their own practices, these beliefs are embedded in a liberal system over which they have no control. In addition, as the arguments of Kymlicka and Tully suggest, this system is itself biased in favour of the liberal majority. This seems to accord with Parekh's view of the political structure of liberal theory.

Parekh's reaction to the limitations of liberalism is to argue that we should adopt an inclusive dialogue that encompasses not only liberal but other beliefs, and that reaches towards universal moral values.

The dialogically constituted multicultural society both retains the truth of liberalism and goes beyond it. It is committed to both liberalism and multiculturalism, privileges neither, and moderates the logic of one by that of the other. It neither confines multiculturalism within the limits set by liberalism and suppresses or marginalizes nonliberal values and cultures, nor confines liberalism within the limits of multiculturalism and emasculates its critical and emancipatory thrust. ${ }^{155}$

Parekh views the dialogue in a multicultural society as involving and reconciling liberalism and multiculturalism. This can be contrasted with the work of Kymlicka, where multicultural issues are grafted onto a liberal structure. For Parekh, both are necessary parts of the overall dialogical process. Overall, Parekh's organising idea is of a dialogue underpinned by both his meta-ethical conception of approaching universal values through dialogue, and the moral value of culture and cultural diversity.

${ }^{155}$ Ibid, p340. 
Kymlicka criticises Parekh's view of liberalism. He argues, firstly, that many of the minorities that western societies deal with are themselves liberal, and, secondly, that in these cases there is not the same need for a dialogue that looks beyond liberalism. ${ }^{156}$ He goes on to say that, even in relation to non-liberal groups, Parekh's conception of dialogue is itself recognisably liberal. He identifies the ground rules that a liberal conception of dialogue would include, such as equal participation, free speech and democratic representation, and argues that Parekh's ground rules are essentially similar.

He rejects any attempt by non-liberal groups to exclude some people from participating in the public debate (e.g. women, blacks or heretics); he rejects any attempt to assert that one group has the natural or divine authority to rule over others (i.e. he requires that authorities have the consent of the people they claim to speak for); he rejects attempts to insulate any group's claims from contestation or criticism. His account of these ground-rules is rather sketchy, but it seems to me to be eminently liberal in its basic inspiration.... Indeed, much of his account of the ground-rules of dialogue seems to be taken almost verbatim from liberal accounts of public dialogue. ${ }^{157}$

Parekh replies to this argument directly, stating that, although there are some common features, essentially, his conception of dialogue is different from that of liberalism.

My approach involves empowerment of weaker groups and voices, a heterogeneous public, a public debate that allows for a multiplicity of voices, and a view of public reason that is subject to constant contestation and refinement. My view does not require citizens to abstract away their cultural and other identities as a condition of citizenship. ${ }^{158}$

The distinction the Parekh draws between his conception of dialogue and liberalism is that the former is more open and inclusive and more identity-sensitive, and the outcome of public reason is dynamic and continually transformable.

This latter claim may differ from earlier liberal approaches, but theories of public reason are, for the most part, similarly dynamic. The other differences are potentially valid, in that Parekh's approach may actually succeed in including more non-liberal ideas. Whether the approaches of Kymlicka and Parekh are essentially different or not, there is a more fundamental problem for him. What Kymlicka's analysis shows is that Parekh's dialogue, whatever its underlying values and justification, amounts to a substantive doctrine in much the same way as liberalism. It involves a substantial set of pre-conditions and moral ground rules, which include a set of universal individual

\footnotetext{
${ }^{156}$ W Kymlicka, 'Liberalism, dialogue and multiculturalism' (2001) 1(1) Ethnicities 128, p129.

${ }^{157}$ Ibid, p132.

${ }^{158}$ B C Parekh, 'A response' (2001) 1(1) Ethnicities 137, p139.
} 
rights, and a broad conception of equality. ${ }^{159}$ It implies a conception of the person, particularly in relation to their culture, and a conception of the just society, as well as the correcting of its moral assumptions about the relationship between dialogue and universal values.

It is also clear that Parekh's approach implies a substantive political culture. This culture amounts to a dialogical political ethic to which all cultural groups must subscribe in order to be involved fully in the political decision-making process. Parekh argues that dialogue will result in a thin societal culture and identity, but that the success of this dialogue, itself, depends on a particular political culture. This culture is, by design, multicultural, and as thin as possible, but it is still a uniform, universal culture; in this sense, moral multiculturalism necessarily collapses into a form of monoculturalism.

Not only is there substantive overlap between Parekh's multicultural dialogue and corresponding liberal approaches, there are also strong structural similarities. Parekh's approach recognises substantial personal (and group) freedom, which is expressed through their role in a fair and equal dialogue. Those who are non-dialogical in this sense are marginalised in exactly the same way that he criticises liberalism for marginalising the non-liberal. Those people are free to express their beliefs within the context of a dialogue, and to benefit from whatever freedom and advantages they can secure through that process; but, they are not capable of questioning and reversing the process itself, just as non-liberals are not capable of having their non-liberal preferences used to undermine the underlying liberal approach.

Parekh may argue that the very rules of dialogue are subject to change and alteration. In the above passage, he states that public reason is dynamic, but this probably refers only to the structure and substance of the public reason. Even if he were to assert that the rules of the dialogue are changeable, as does Gaus of his public justification, this would apply only minimally to some of the conditions. In other words, it can mean changing some of what is meant by fair and equal dialogue, but cannot change the fact that fair and equal dialogue is the appropriate political approach.

\footnotetext{
${ }^{159}$ See above, notes $147,148$.
} 
In that liberalism and Parekh's dialogical approach both encourage freedom and pluralism within specifically set boundaries, both face the burden of justifying their specific boundaries. In this, Parekh's attitude towards his conception of public multicultural dialogue seems to be that it is the best approach for modern western liberal and multicultural societies. This means that it is supposed to apply not only to liberal majorities, but also to non-liberal and non-dialogical minorities. Non-dialogical groups would include not simply those who are positively antithetical to conversation and co-operation, but, also, those that reject Parekh's conceptions of individual equality, respect and political access.

There seem to be both structural and substantive similarities between liberalism and Parekh's theory. The rationale for Parekh's dialogue depends on a conception of multicultural inclusion, and on, both, the relationship between individuals and culture, and, the value of diversity. Each of these elements might reasonably be described as liberal, in that each involves respect, and the presumption that freedom should extend to personal beliefs and choices. Parekh's focus does differ from that of Kymlicka's multiculturalism, and Gaus' public dialogue, by virtue of being more willing to treat groups as valid political units; but, this does not prevent his theory from being essentially liberal. On this view, what Parekh's approach offers liberal theory is a focus on cultural inclusion and respect as the central liberal concerns, so that, in actual political interaction, cultural arguments are capable of being treated on the same level as difference-blind liberal doctrine.

In order to justify this approach, Parekh would need to recognise explicitly its two levels, and to distinguish between the underlying liberal (or dialogical) values, and the liberal substance of political dialogue. Parekh fails to explain why these values are not subject to the same criticisms he levels against doctrinal liberalism. From the perspective of moral theory, he fails to explain why these underlying values are presented by him through theory, and are not subject to dialogical justification. Ultimately, it seems that Parekh's approach contains exactly those problems that he rightly attributes to liberalism, and, so, although it develops liberal theory in relation to multiculturalism in an interesting and potentially meaningful way, it fails to solve the fundamental problems of liberal justification. 


\section{Conclusion}

Multicultural demands present an important challenge to liberal theory. The substance of this challenge is that individual pluralism is of a different kind than cultural pluralism, such that the latter cannot be adequately accommodated by difference-blind theories that prescribe universal individual rights. The basis for this difference is in the fundamental importance of culture to our identities and selves; at the most basic level, the central Rawlsian freedom to choose one's conception of the good does not take into account the dependence of this conception on culture. Instead, the recognition of minority cultures within a liberal society requires, it is argued, culturesensitive principles that reflect the preference of liberal rights for one cultural group over another.

Multiculturalism, then, is unique among the perspectives on liberalism discussed here in that it opens liberalism to rights that are differentiated between peoples within a society. This move is opposed by those liberals who fear that group rights would undermine the impartiality of difference-blind liberalism and open liberal politics up to group-focussed arguments, based on interest rather than justice. This reticence is probably reflected in Kymlicka's attempts to graft a particular set of multicultural rights onto a liberal framework. In contrast, Tully and Parekh are more willing to explicitly reject accepted forms of liberalism as being universal, and, instead, to treat liberalism as one cultural part of a dialogue of cultures and, so, as one doctrine, to be respected by virtue of its advantages and its popularity, among many others.

Each of these approaches faces a number of difficulties. Firstly, the theories, by virtue of reflecting an actual political movement, need to be able to encompass the many circumstances of multiculturalism in western countries; but, more importantly, from a theoretical standpoint, the overall conception of culture itself must not only reflect this diversity, but, also, must express culture as a different, special kind of pluralism. This task involves: expressing an understanding of culture that includes its constitutive value to individual identities; while, at the same time, allowing for the necessary 
diversity and dissent internal to a culture, the diversity of forms of cultural attachment, the dynamics of cultural development, and the interactive, interdependent relationships between cultures. To the difficulty of this project must be added, Scott's suggestion that culture itself is a historically-situated idea, so that, even the universality of culture is questionable. Overall, none of the surveyed theories manages to incorporate all of these elements in a meaningful way, although Parekh's conception of individuals mediated by culture is probably the closest to the mark.

The next challenge that these theories face is the inevitable tension between culture and justice. In an explicitly liberal theory such as Kymlicka's, this manifests most obviously in the case of illiberal national minorities. In this case, there is a conflict between a culturally conservative approach, which would dictate that the liberal majority allow the minority to continue in their illiberal ways, and the liberal values to which the majority subscribe. To favour the former would be to allow merely cultural values to override liberal values, while to enforce a liberal approach would be to impose majority ideals on a culturally distinct minority. Neither is very attractive: the former suggests that liberalism is simply the conventional expression of western political history, and is not relevant for non-westerners; the latter asserts the more universal character of liberal values, which undermines the very nature of cultural pluralism. Kymlicka attempts to find a middle-ground between the two. He distinguishes between identifying a valid theory of multiculturalism and imposing it on minorities, and argues that the latter should not occur unless the minority breach of human rights is extreme. This argument is thoroughly unconvincing, and seems to emphasise the difficulty involved, rather than take any positive step towards answering it.

It might be expected that Parekh and Tully would respond to this challenge much more effectively, given that their approaches both imply a willingness to favour multicultural over liberal values. In fact, both face similar difficulties to Kymlicka, as both depend on particular universal theories of multicultural justice. Both champion those cultural beliefs that are adhered to by minorities, while also promoting universal values that are multicultural and/or liberal. While it might be argued that there is a natural affinity between minority cultures and multiculturalism, this is no more than the affinity between autonomous individuals and liberal toleration. The point is that 
minority groups may not agree with or adopt a particular conception of multiculturalism, or even the meaning of, or need for, multiculturalism itself. The potential for conflict between cultural values and multicultural values, then, undermines their theories in the same kind of way as the conflict between culture and liberalism undermines Kymlicka's. And, even if it is argued that minority cultures would tend to prefer a more culturally-sensitive and less difference-blind approach, this rests on group interests rather than the needs of justice, and involves satisfying minorities only to the extent that it takes away from majorities. It also ignores the diversity of both minority and majority cultures.

The final challenge to multicultural liberalism is closely linked to this conflict, and entails effectively conceiving of the cultural bias of liberal theory. The earlier chapters found, implicit in liberal theory, a source of bias, namely the assertion of particular and plausible ideas and values within a diverse and contestable space.

Multiculturalism views liberal bias from the perspective of culture, and finds it, not only in some of the assertions of theorists, but, also, in the very way that liberalism operates within the political domain. Kymlicka suggests this in relation to a number of political decisions that had previously not been the concern of liberalism, and, so, furthers his project of adding group rights to an otherwise untouched liberal framework. Tully and Parekh take this bias further, and label all of liberalism as being doctrinal and questionable.

Ultimately, this is the greatest multicultural challenge and contribution to liberal theory: that aspects of the theory itself, conceptions, assumptions, values and principles are potentially culturally biased. In this way, multiculturalism opens liberalism up to a particular expression of moral uncertainty, namely that moral conclusions are the result of cultural circumstances. This sets a substantial burden on liberal justification, given that the nature and extent of this bias is expressed only in the most general terms, and the means for correcting for it are difficult to conceive. The one obvious response to this difficulty is to employ a justification that is culturally contextual, but any conception of culture would undermine such an attempt. Multiculturalism itself renders a culturally-based justification only partially useful, and the internal diversity of cultures suggests that it would be difficult to apply such a justification to the entirety of a culture. In this way, the nature of the difference 
implied by multiculturalism undermines a justification based on some common, albeit contextual, element.

The responses of Parekh and Tully to this problem are to use as their central focus a conception of multicultural society. This conception, itself limited to modern western states, leads to their respective ideals of intercultural dialogue, which share much with liberal conceptions of public reason and deliberative democracy. These dialogues involve interaction between cultures, and between liberalism and other moral and political beliefs. Despite liberalism's role as a subject in the dialogue, the conditions and values that underlie dialogue itself are, in many ways, recognisably liberal, albeit from a multicultural perspective. It turns out, then, that, although the dialogical interaction between liberal and other theories within a multicultural system is interesting, what is perhaps more interesting is the interaction between liberal and multicultural values that underlie that dialogue. This is most obvious for Parekh, who explicitly rejects the universality of liberalism, but builds his approach on an unquestioned application of a conception of multicultural liberalism, or perhaps, liberal multiculturalism.

The attempt at devising a conception of justice that transcends cultural differences, by being centrally focussed on the fact of cultural pluralism, and by being substantively respectful of and open to various cultural expressions, is undermined by the particular moral values and approaches that express this focus. As with pluralism, the fact of multiculturalism does not seem to necessarily imply liberal multiculturalism. The latter amounts to simply one possible response to the former, albeit one that is sensitive to the need to respect individuals and cultural groups, and that focuses on cooperation and mutual recognition.

The perspective of multiculturalism offers a deeper view of this idea. For the dialogical approaches of Tully and Parekh to be successful, the cultural groups involved have to adopt particular cultural beliefs about political interaction and intercultural dialogue. Much like the reasonableness requirement of public reason and political liberalism theory, neither Parekh's nor Tully's dialogues function unless those groups involved value highly multiculturalism and cultural diversity, and are willing to interact on a co-operative basis. It could be argued that these beliefs 
themselves form part of the theory's justificatory context, and that, once they are admitted, the intercultural dialogue becomes the best possible moral and political approach in the circumstances. This move would certainly make a successful transcultural justification possible, but it entails a move that contravenes the essence of multicultural demands; it involves imposing a particular set of cultural beliefs on minority cultures. Not only must all cultures accept the relevant liberal-multicultural values, but they must hold them as being morally or, at the very least, politically, fundamental.

This amounts to a form of cultural homogenisation that seems biased like any other, regardless of the fact that it is based on ideas of multiculturalism. It might be argued that this bias corrects for or balances other biases inherent in political activity. This may be correct; however, this entails imposing cultural and moral content on the basis of justice, which merely emphasises the tension between culture and justice discussed above. It is difficult to see why this multicultural/liberal approach should direct cultural belief and political justice when liberal values on their own are consigned to the status of contestable doctrine.

Overall, multiculturalism has influenced liberal theory: by adding a cultural dimension to its operation and, also, in alerting the theorist to the operation of cultural and liberal bias. In this way, multicultural theory can be seen as a strategy for overcoming the moral uncertainty expressed in cultural difference. This shift in the aims of liberal theory is unable to reach a transcultural perspective, and, instead, amounts to an ethic of cultural sensitivity, embedded in multicultural liberal theory, public political culture, and intercultural political dialogue. What remains is an unresolved tension between liberalism as a western cultural tradition, and multicultural liberalism as the best conception of justice for western societies. 


\section{POSTMODERNISM}

\section{Rorty's postmodern liberalism}

Multicultural theory accuses liberalism of cultural bias, and prescribes a more inclusive liberal public dialogue between majority and minority cultures. Postmodernism also accuses liberal theory of bias, and, like multiculturalism, it emphasises generally the historical and cultural location of liberal theory within the western tradition of political theory and action. Unlike multicultural demands, the basis for a postmodern critique of liberalism extends to a close examination of theoretical methods, and, so, envisages a liberal bias that extends beyond culture to the most basic features of theory itself.

Postmodernism has been described as involving incredulity towards the "supposedly transcendent and universal truths that underpin western civilization". ${ }^{1}$ More than this, postmodern theorists tend to subscribe to beliefs and approaches that might be described as being radically sceptical, and they are often labelled as relativists, subjectivists or emotivists. An examination of postmodern approaches to liberalism is potentially interesting in that such approaches would tend to take moral uncertainty as their starting-point, and, presumably, would be in a strong position to build a liberalism that responds well to this uncertainty. The difference between postmodern and other liberal theorists is that the former interact with this uncertainty in an immediate and self-conscious way.

As suggested in the introductory chapter, there are two general difficulties with a thoroughly morally sceptical liberal theory. The first is that this scepticism is potentially self-refuting, in that a statement undermining absolute claim, seems itself to be an absolute statement, and, so, is subject to the same argument. The second is that a theory that prescribes positive liberal values is seemingly at odds with the

${ }^{1}$ J W Bertens, The Idea of the Postmodern: A History (1995), p124 
incredulity towards any universal moral judgment. A theory of postmodern liberalism must overcome both of these obstacles in order to be valid as a response to uncertainty.

The primary attempt at building a postmodern form of liberalism is to be found in the work of philosopher Richard Rorty. His book, Contingency, Irony, and Solidarity, published in 1989, attempts to apply, to the liberal realm, his criticisms of the idea that knowledge is "a matter of mental or linguistic representation of reality". ${ }^{2} \mathrm{He}$ expands his views in subsequent essays, collectively published in Objectivity, Relativism, and Truth in 1991, and also in a later collection, Philosophy and Social Hope (1999). ${ }^{3}$ These works develop various aspects of a relatively consistent view of the nature of philosophy and the value of liberalism.

\subsection{Contingency and reality}

Perhaps the most significant idea that Rorty uses to describe people and their relationship to the world in which they live is that of contingency. Keith Topper has described Rorty's conception of contingency, broadly speaking, as embodying the idea that things might have been otherwise, as opposed to being "necessary, essential, intrinsic, or unconditional". 4

These latter terms... are themselves tightly intertwined with notions of a common "human nature," a telos, a divine order or some other principle of legitimacy that is privileged precisely because its status is independent of our particular historical location and social practices. $^{5}$

Rorty's philosophy, then, is based on a retreat from the necessary or essential character of reality, of people, beliefs, and ideas. Instead, he emphasises the dependence on geographic, social and linguistic location. Topper notes that this means the championing of "novelty, innovation, originality, and creativity", but, at the

\footnotetext{
${ }^{2}$ R Rorty, Contingency, Irony, and Solidarity (1989); T Mautner (ed.), The Penguin Dictionary of Philosophy (2000), pp488-489, entry written by Richard Rorty.

${ }^{3}$ R Rorty, R, Objectivity, Relativism, and Truth (1991); R Rorty, Philosophy and Social Hope (1999).

${ }^{4}$ K Topper, 'Richard Rorty, Liberalism and the Politics of Redescription' (1995) 89(4) American Political Science Review 954, p958, emphasis removed.

${ }^{5}$ Ibid.
} 
same time, "chance, luck, accident, randomness, and fortuitousness". ${ }^{6}$ As contingency is one of the central concepts used by Rorty, these ideas are values that are woven through much of his theory.

Rorty's first use of this contingency is in explaining the nature of the relationship between people and the physical world, and, so, the meaning of our understanding of reality. He describes his position as being anti-representational, meaning that our linguistic descriptions of the world do not in any way amount to accurate representations of it, and that any view contrary to this is pointless. ${ }^{7}$ In this way, truth is made and not found, and, so, is subject to the contingencies of the constructed: ${ }^{8}$

\begin{abstract}
Since truth is the property of sentences, since sentences are dependant for their existence upon vocabularies, and since vocabularies are made by human beings, so are truths. For as long as we think that "the world" names something we ought to respect as well as cope with, something personlike in that it has a preferred description of itself, we shall insist that any philosophical account of truth save the "intuition" that truth is "out there." This institution amounts to the vague sense that it would be hybris on our part to abandon the traditional language of "respect for fact" and "objectivity" - that it would be risky, and blasphemous, not to see the scientist (or the philosopher, or the poet, or somebody) as having a priestly function, as putting us in touch with a realm which transcends the human. ${ }^{9}$
\end{abstract}

So, for Rorty, there is no priestly and privileged connection between our descriptions of reality and reality itself. This idea renders truth, as expressed by language, as constructed out of whatever vocabularies we happen to exercise. This antirepresentational approach applies the contingency of both language and truth, and holds that our expressions about reality offer no inkling of a real world above and beyond those expressions themselves. In this way Rorty claims to de-divinise the world - to deny its transcendent and absolute character and, instead, to locate it relative to those who are describing it. ${ }^{10}$

It might be argued in response to Rorty's anti-representationalism that some descriptions, such as the existence of a physical object, or a particular physical law, actually reflect reality. He replies to this position by arguing that the contingency of constructed truth applies to physics just as it does to astrology or literary criticism, and that what appears as hard fact is actually "simply the hardness of the previous

\footnotetext{
${ }^{6}$ Ibid, pp958-959.

${ }^{7}$ Rorty, op cit, above note 2, p4.

${ }^{8}$ Ibid, p3.

${ }^{9}$ Ibid, p21.

${ }^{10}$ Ibid.
} 
agreements within a community about the consequences of a certain event". ${ }^{11}$ What, if anything, distinguishes physics and literary criticism, then, is the firmness with which the relevant communities determine "who loses and who wins". ${ }^{12}$ The virtue of scientists, Rorty suggests, lies not in an assertion to truth, but in the success of the institutions to which they adhere and the "unforced agreement" that results. ${ }^{13}$

What is important for Rorty is not just the contingency of our descriptions of reality, but to show a relationship between contingency and pragmatism, the latter being one of his central substantive principles. People are not conceived of as being in a freefloating and reality-less state, but, rather, with respect to their performance within an environment. In this way, linguistically-expressed beliefs define not what reality is, but how people interact with their environments. ${ }^{14}$

\begin{abstract}
Pragmatists hope to break with ... the Cartesian-Lockean picture of a mind seeking to get in touch with a reality outside itself. So they start with a Darwinian account of human beings as animals doing their best to cope with the environment - doing their best to develop tools which enable them to enjoy more pleasure and less pain. Words are among the tools which these clever animals have developed. ${ }^{15}$
\end{abstract}

So, for Rorty, the constructedness of belief-statements is not meaningless, but, rather, allows people to interact effectively with their environments. On this view, it does not matter whether these beliefs represent the world accurately. What is important is that they allow us to accomplish particular tasks, or, generally, to lead good lives. ${ }^{16}$ In this context, the truth of a belief means simply that "no alternative belief is, as far as we know, a better habit of acting". ${ }^{17}$

Rorty goes on to characterise the human mind as being a web of these beliefs and desires, from which there is no distinct self. ${ }^{18}$ The self, then, has no unchanging centre, but, rather, is made up of our beliefs and desires "in continual interaction, redistributing truth-values among statements". ${ }^{19}$ This means that there is no common

\footnotetext{
${ }^{11}$ R Rorty, Objectivity, Relativism, and Truth (1991), pp8, 80.

12 Ibid.

13 Ibid, p39.

${ }^{14}$ Ibid, p10.

${ }^{15}$ R Rorty, Philosophy and Social Hope (1999), ppxxii-xxiii.

${ }^{16}$ Ibid, Pxxiv.

${ }^{17}$ Ibid, Pxxv.

${ }^{18}$ Rorty, op cit, above note 11, p93.

${ }^{19}$ Ibid, p123.
} 
human nature and, so, just like the world, the self is de-divinised, and treated as the accidental result of contingent construction. ${ }^{20}$

\subsection{Postmodernism and theory}

Because of his claims as to the contingency of belief, and his rejection of any method aimed at universal results, Rorty is often denounced as a relativist. ${ }^{21}$ It is true that he views language and belief only as pragmatic customs, and argues that there is no external context-independent viewpoint from which to perceive them. Yet, he both rejects the relativist label, and declares the "need to avoid relativism" to be "an expression of the need to preserve certain habits of contemporary European life". ${ }^{22}$ Thus, he reduces this criticism, and, by inference, western attempts at moral universalism, to a misleading or even underhanded statement on the importance of retaining certain contingent western values. He later expresses the western ideal of a universal truth rather more charitably as expressing, in a misleading way, the hope that "the human race as a whole should gradually come together in a global community... which incorporates most of the thick morality of the European industrialized democracies". ${ }^{23}$ Rorty's message here is expressed in the same terms as before; it is just that, here, he agrees with the corresponding pragmatic instinct, namely to broaden the moral community.

Rorty does not identify himself as a relativist, and finds the distinction between the absolute and the relative to be clumsy. He argues that, along with the philosophical distinctions between objectivity and subjectivity, and between facts and values, the distinction between the absolute and the relative should be discarded and replaced by a new vocabulary. ${ }^{24}$ This leads Rorty to characterise pragmatists like himself as antidualists, unwilling to accept a particular group of dualities that pervade western philosophy and common sense. ${ }^{25}$

\footnotetext{
${ }^{20}$ Rorty, op cit, above note $2, \mathrm{p} 40$.

${ }^{21}$ Rorty, op cit, above note 15 , pxvi.

${ }^{22}$ Rorty, op cit, above note 11,p28.

${ }^{23}$ Rorty, op cit, above note 15 , pxxxii.

${ }^{24}$ Ibid, ooxxvii-xix; Rorty, op cit, above note 11, p36.

${ }^{25}$ Rorty, op cit, above note 15 , pxix.
} 
Without the possibility of a standpoint that is external to any moral community, Rorty must employ an approach to theory and justification that amounts to a redescription of universalist approaches. His interpretation of current practice is that knowledge and truth represent common agreement within a particular linguistic community. ${ }^{26}$ Similarly, approaches to justification reflect familiar procedures accepted within a particular field. ${ }^{27}$ He suggests that there is no method of ultimate justification to which we can appeal, and that theory is simply another text and, so, cannot claim a privileged status. ${ }^{28}$ It is this theoretical landscape in which Rorty is tasked to justify, or at least to explain, liberalism. It is characterised by the contingency of belief and of self; by the inescapable contextual essence of ideas and thought. In it, all reference is made to individual's webs of beliefs, and to the social and linguistic communities of these individuals. Their habits and customs are the only perspective from which moral theories can be judged. Congruent with these ideas, Rorty describes philosophy as "an aid to creating ourselves", rather than knowing ourselves; and morality as a "new and controversial custom". 29

This is not to deny that theory or morality cannot improve society, for such a claim would imply the relativist idea that all communities' values and beliefs are equal. This evaluation, Rorty argues, is impossible since it implies a perspective beyond context. ${ }^{30}$ Instead, the point is to accept that philosophy occurs within a particular tradition, and not to attempt to theorise from a perspective beyond that tradition. ${ }^{31}$ This does not prevent people from adhering strongly to moral values, or from holding beliefs that they consider are worth dying for. ${ }^{32}$ However, it does imply a highly contextualised approach, where the subject of philosophy is as contingent as Rorty finds language or the self.

\subsection{Contingency and liberalism}

\footnotetext{
${ }^{26}$ Rorty, op cit, above note 11, pp23-24; Rorty, op cit, above note 15, p15.

${ }^{27}$ Rorty, op cit, above note 11, p23; Rorty, op cit, above note 15, p36.

${ }^{28}$ Rorty, op cit, above note 11, p79; Rorty, op cit, above note 15, p28.

${ }^{29}$ Rorty, op cit, above note 15, pp69, 76.

${ }^{30}$ Rorty, op cit, above note $11, \mathrm{p} 202$.

${ }^{31}$ Ibid.

${ }^{32}$ Rorty, op cit, above note 2, p189.
} 
Within this framework of contextuality, Rorty is tasked with promoting a conception of liberalism. The issue is how this promotion can take place given his objection to the aspiration to universality, and even to the idea that philosophy is conventional and cannot transcend linguistic communities. Rorty's approach to liberalism eschews justification, in favour of a process of redescription. The latter is undertaken in order to "reformulate the hopes of liberal society in a nonrationalist and nonuniversalist way". ${ }^{33}$ This process involves replacing conceptions of rationality and truth with ideas of metaphor and self-creation, and, so, creating a liberal culture that is de-divinised and that responds to the internal needs of its society, rather than to external ideals such as holiness or reason. ${ }^{34}$ For this reason, liberalism needs no philosophical justification at all, although philosophy and theory can be used to build a theory of social justice that accords with accepted beliefs and institutions. ${ }^{35}$

It is clear, then, that Rorty is not attempting a "transcendental deduction of democratic politics from anitrepresentationalism premises". ${ }^{36}$ Nor does a commitment to pragmatism entail a similar commitment to liberalism. ${ }^{37}$ Instead, he hints that political theory "should view itself as [offering] suggestions for future action emerging out of recent historical experience, rather than [as] attempting to legitimate the outcome of that experience by reference to something ahistorical". ${ }^{38}$ These ideas confirm the radically contingent nature of any attempt at liberal theory, and raise obvious challenges for a person such as Rorty who seeks to argue in favour of liberalism; as, without a transcontextual criterion or viewpoint, it is difficult to see what form these arguments can take or how powerful they can be. In order to explore these issues, the substance of Rorty's liberal theory needs to be examined.

At the centre of Rorty's liberalism is the separation between the personal desire for self-creation, and the public need for solidarity, defined as Judith Shklar's liberal ideal of the avoidance of cruelty. ${ }^{39}$ Rorty's liberalism is the embodiment of the idea that

\footnotetext{
${ }^{33}$ Ibid, p44.

${ }^{34}$ Ibid, pp44-45.

${ }^{35}$ Rorty, op cit, above note $11, \mathrm{p} 178$.

${ }^{36}$ Ibid, p15.

${ }^{37}$ Rorty, op cit, above note $15, \mathrm{p} 271$.

${ }^{38}$ Ibid, p272.

${ }^{39}$ Ibid, pxv.
} 
these two values are "equally valid, yet forever incommensurable". ${ }^{40}$ This means they cannot be effectively combined, nor can one be found to be morally prior to the other. These two values, the relationship between them, and the society that results from the equal respect of both, make up the substance of Rorty's liberalism.

Self-creation in Rorty's theory is intimately connected with being an ironist. Such a person has personal doubts about their own fundamental beliefs and justifications, recognises that these doubts cannot be removed simply by applying their current vocabulary, and does not believe their vocabulary to be any better a reflection of reality than that of anyone else. ${ }^{41}$ In other words, they accept the contingency of themselves, their cultures and societies, and their beliefs. By virtue of these beliefs, an ironist is in the best position to engage in a process of autonomous self-creation, with the aim of making "the best selves for ourselves that we can". ${ }^{42}$

In this way, Rorty champions the idea of continual self-redescription, but a redescription that is free of the metaphysical urge to theorise; this is a process that self-consciously originates within a particular tradition, or within the intersection of a number of traditions, but that attempts to push at the boundaries of the vocabularies these traditions offer. ${ }^{43}$ This self-conscious self-creation, he argues, characterises the citizens of a liberal utopia founded on the acceptance of contingency. ${ }^{44}$ Importantly, it requires some basic personal freedoms.

The social glue holding together the ideal liberal society... consists in little more than a consensus that the point of social organization is to let everybody have a chance at selfcreation to the best of his or her abilities, and that the goal requires, besides peace and wealth, the standard "bourgeois freedoms." This... would be a conviction based on nothing more profound than the historical facts which suggest that without the protection of something like the institutions of bourgeois liberal society, people will be less able to work out their private salvations, create their private self-images, reweave their webs of belief and desire in the light of whatever new people and books they happen to encounter. ${ }^{45}$

The demands of self-creation, Rorty argues, imply the 'standard bourgeois freedoms' and institutions. He asserts that the normative underpinnings of liberal society need consist of no more than the belief that all should be afforded the opportunity for self-

\footnotetext{
${ }^{40}$ Rorty, op cit, above note 2 , pxv.

${ }^{41}$ Ibid, p73.

42 Ibid, p80.

${ }^{43}$ Ibid, pp97-99.

${ }^{44}$ Ibid, p61.

${ }^{45}$ Ibid, pp84-85.
} 
creation. This actual, contingent belief acts as the link between self-creation and a set of individual liberties.

The other part of Rorty's conception of liberalism is expressed by the need for solidarity, or the fundamental importance of avoiding cruelty within a society. $\mathrm{He}$ expresses explicitly the idea that there is no non-neutral theoretical justification for the idea that cruelty is the worst thing that can happen in society. ${ }^{46}$ Yet, he argues that there is, at the most basic level, a solidarity between people that expresses a common humanity, and withstands the limitations implied by contingency. ${ }^{47}$ This solidarity does not function universally, but, rather, depends on the beliefs of individuals and groups, and, in particular, on how the potential subject of solidarity is perceived by the person or group. ${ }^{48}$

So, the relationship of solidarity between two people or groups depends on the contingent beliefs of those groups. Moral progress, then, is achieved by the increasing of feelings of solidarity.

But that solidarity is not thought of as recognition of a core self, the human essence, in all human beings. Rather, it is thought of as the ability to see more and more traditional differences... as unimportant when compared with similarities with respect to pain and humiliation. $^{49}$

It is this increased solidarity, and the corresponding decrease in pain and humiliation, that should be the proper goal of a liberal society. This is based on the recognition, not of a common humanity, but of the effect of cruelty that is common between people regardless of differences in belief.

A system that treats equally the demands for autonomous self-creation, and for solidarity expressed as the avoidance of cruelty, would satisfy Rorty's conception of a just and liberal society. This society would feature both the basic liberal freedoms and the active minimisation of cruelty. These values would be pursued in the public realm "by persuasion rather than force, by reform rather than revolution, by the free and open encounters of present linguistic and other practices with suggestions for other

\footnotetext{
${ }^{46}$ Ibid, ppxv, 197.

${ }^{47}$ Ibid, pp189-190.

${ }^{48}$ Ibid, pp191-192.

${ }^{49}$ Ibid, p192.
} 
practices". ${ }^{50}$ Rorty argues that, given the choice between a liberal society that satisfies these conditions, and an illiberal society, no-one who has experienced both would choose the latter. ${ }^{51}$ Such a liberal society, Rorty argues, is likely to result in an increase in human happiness. ${ }^{52}$ To pursue these ends, on pragmatic grounds, is for Rorty the equivalent of substituting hope for knowledge as the centre of human endeavour. $^{53}$

\section{Analysis}

\subsection{Theory and contingency}

The structure of Rorty's account of liberalism is founded on the general application of the postmodern ideal of contingency, and, so, on the relegation of moral values and theory to the realm of custom, rather than to that of universal truth. His conception of liberalism itself comprises two parts: self-creation as ironism, and solidarity as the liberal avoidance of cruelty and humiliation. These two aspects are supposed to represent the primary values of the private and public areas of our lives respectively, and are to be treated as equal and incommensurable within a liberal system. As such, they represent a liberal utopia that is epitomised by the liberal ironist, who is autonomous, and yet conscious of contingency in his or her personal life, and, who, in the public domain is an ardent supporter of a politics characterised by the liberal avoidance of cruelty.

Given Rorty's postmodern and pragmatist viewpoint, and his renouncement of theory and justification, it is not clear the grounds on which he promotes this liberal utopia, or what this explanation requires. His approach is not entirely distinct from contemporary liberal theory, and has similarities to both value pluralism and political liberalism: the former, in the focus on the historicity and cultural location of political theory and belief, and the pluralisation of moral value; and the latter, in the

\footnotetext{
${ }^{50}$ Ibid, p60.

${ }^{51}$ Rorty, op cit, above note 11, p29.

${ }^{52}$ Rorty, op cit, above note 15, pp270, 276.

${ }^{53}$ Ibid, p88.
} 
subordination of philosophy to the political. ${ }^{54}$ Yet, Rorty sets aside the need for justification in a fundamental way. Without the requirements of justification and theory it is not obvious what he is attempting to achieve, in setting out, in a seemingly theoretical way, a structure that is supposed to represent a liberal utopia.

According to Rorty's account of our contingent reality, morality is founded entirely on custom. This means that moral theory cannot recognise any moral truth, but rather is part of the process of the construction of that truth. On this view, theory is one among many texts, characterised by the focus on ideas rather than events or people. ${ }^{55}$ Theory that is self-consciously contingent, then, discards the search for proper foundations and, instead, acts as a tool to modify or develop its subject. The method by which this is achieved is through the redescription of the subject's vocabulary. ${ }^{56}$ This allows shifts in individual belief, since this is expressed in and mediated by language. In this way, Rorty's objections do not deny completely the role of philosophy and justification, but, rather, deny that they can be used in a foundational way in order to reveal true and universal principles.

One way of formulating the pragmatist position is to say that the pragmatist recognises relations of justification holding between beliefs and desires, and relations of causation holding between these beliefs and desires and other items in the universe, but no relations of representation. Beliefs do not represent nonbeliefs. ${ }^{57}$

It is wrong, then, to criticise Rorty for using theory to promote liberalism, without examining the substance of that theory. The latter should be recognised, consistent with the remainder of Rorty's ideas, as attempting a non-foundational redescription of liberalism that does not represent, but, rather, reinterprets and recontextualises a liberal reality.

An important and fundamental criticism remains about Rorty's general theory: given that Rorty has characterised theory as being contingent in this way, it is necessary for him to explain why his assertions of contingency are not themselves contingent. This represents the first challenge of sceptical theory, that the assertion of scepticism is self-refuting, since it is itself a universal and, so, non-sceptical claim.

\footnotetext{
${ }^{54}$ Rorty, op cit, above note 11, p185; see also J Fennell, 'Harry Neumann and the Political Piety of Rorty’s Postmodernism' (1999) 26(2) Interpretation 257, p259.

${ }_{55}^{5}$ Rorty, op cit, above note $15, \mathrm{p} 79$.

${ }^{56}$ See Rorty, op cit, above note 2, p39.

${ }^{57}$ Rorty, op cit, above note 11, p97.
} 
This is potentially damaging to Rorty's approach. Either, the assertion of contingency is itself subject to that contingency, or, it stands above and transcends it. Neither possibility seems coherent with Rorty's account of theory and reality. The first means that contingency is not universal and is itself limited to a particular (postmodern and pragmatic) tradition. This in itself implies a higher level contingency that the idea of contingency denies, and would offer no reason for preferring contingency to some other approach. The second is no less troublesome, for the truth of contingency seems to belie the consequences of that truth, that there is no truth except what is constructed by us in order to deal with our environment. This makes the assertion of truth exactly the kind of statement that Rorty denies that other philosophers and theorists can make.

This is further explicated by Peter Lom's criticism that Rorty's contingency is itself a metaphysical position.

\begin{abstract}
Rorty would like to remain agnostic on metaphysical questions.... However, he departs from this neutrality on this question of truth in claiming that the ironist is dedicated to two other premises: she is a "nominalist and a historicist."... These... two premises are in tension with [ironist doubt] for they are not subjected to question; moreover, they themselves point to particular metaphysical positions. Historicism and materialism are accompanied by the metaphysical assumption that morality is not underwritten by the nature of the cosmos, that man is entirely alone. ${ }^{58}$
\end{abstract}

Lom suggests that Rorty's attitude of ironism, the awareness of pervasive contingency, includes "assumptions that reveal themselves to be based on undefended and seemingly unexamined premises". ${ }^{59}$ The general point is that, not only is there a logical inconsistency inherent in the assertion of contingency, but that, Rorty's conception of contingency rests on particular contestable and, so, contingent, metaphysical assumptions. Both points, together, suggest that Rorty's assertion of contingency is no less constructed and flawed as any other piece of purportedly transcendent philosophy.

Rorty's likely response to this claim can be pieced together from parts of his explanation of contingency. He states that

\footnotetext{
${ }^{58}$ P Lom, 'East Meets West - Jan Patocka and Richard Rorty on Freedom' (1999) 27(4) Political Theory 447, p452.

${ }^{59}$ Ibid.
} 
[t]o say that we should drop the idea of truth as out there waiting to be discovered is not to say that we have discovered that, out there, there is no truth. It is to say that our purposes would best be served by ceasing to see the truth as a deep matter... "The nature of truth" is an unprofitable topic... [b] ut this claim about relative profitability, in turn, is just the recommendation that we in fact say little about these topics, and see how we get on. ${ }^{60}$

He says elsewhere, when responding to the claim of being a relativist that

the pragmatist is not holding a positive theory which says that something is relative to something else. He is, instead, making the purely negative point that we should drop the traditional distinction between knowledge and opinion, construed as the distinction between truth as correspondence to reality and truth as a commendatory term for well-justified beliefs. $^{61}$

And, in discussing the relationship between postmodernism and relativism, Rorty explains that:

Relativism certainly is self-refuting, but there is a difference between saying that every community is as good as every other and saying that we have to work out from the networks we are, from the communities with which we presently identify. Postmodernism is no more relativistic than Hilary Putnam's suggestion that we stop trying for a "God's-eye view" and realize that "We can only hope to produce a more rational conception of rationality or a better conception of morality if we operate from within our tradition."

These passages, together, suggest a number of points about Rorty's conception of contingency. The first is that it is not a positive assertion of truth, but, rather, a negative ideal: a dropping of the approaches of other theorists for reason of their contingency. This suggests that contingency may itself be viewed as being a selfconsciously contingent response to the philosophical tradition of universality.

The above objection to the idea of admitting the contingency of contingency is that it implies a higher-level contingency that is itself a positive truth claim. The first of the passages above suggests that the higher level principle is in fact pragmatic in nature. In other words, we should adopt a contingent attitude towards philosophy and theory because it is the most profitable and useful in our situation, which would include that other philosophical approaches, particularly universal approaches to morality, have proven to be unconvincing and unhelpful. Thus, contingency is the best approach to take in the situation in which we find ourselves. This seems to be the most faithful portrayal of Rorty's position, but, even these arguments cannot dull the claim that his contingency is self-refuting. The idea that his conception of contingency is a negative principle, enjoining us from engaging in universalist philosophy, must also be positive

\footnotetext{
${ }^{60}$ Rorty, op cit, above note $2, \mathrm{p} 8$.

${ }^{61}$ Rorty, op cit, above note 11, pp23-24.

${ }^{62}$ Ibid, p202.
} 
in that it entails an attitude of ironism, and a working from our own tradition. In addition, the recognition of the contingent nature of contingency implies a higherlevel view of reality that incorporates both the possibility of contingency, and the value of pragmatism.

It is almost as if Rorty imagines a reality of infinite possibility and plasticity, in which one can create without bound, and where the only criterion of the worth of that creation is its usefulness to its creator. In such a reality, given our western tradition and the moral and philosophical uncertainties that have evolved through and as a response to two and a half thousand years of concerted effort, the view of contingency seems, to Rorty at least, to offer the greatest real hope for agreement and happiness. This reality, however, is not what remains after all the false assertions of universality in theory and belief are removed. Rather, it is an imagining of Rorty that presupposes pragmatism and contains within it the seeds of contingency. In other words, it makes contestable metaphysical assumptions which, although they contain an intuitive plausibility as to how the most general level of reality might be, contravene the very process of paring back assertions of universality to an open core. This problem is contained entirely in the direction to stop looking for a God's-eye view: either this suggestion is universal and, so, God-like, or it is not and, so, can offer no reason to do so. Rorty might appeal to pragmatism for such a reason; but then pragmatism, if it could, would take up the role of a God's-eye view.

This is a problem and one that it is difficult for Rorty to overcome without either changing his stance in relation to antirepresentation and truth, to allow contingency to correctly reflect reality, or reassessing the nature of his underlying view of pragmatism and contingency. Ultimately, the claims that contingency is negative and is not tethered to any deeper understanding of reality must fail, since Rorty's approach clearly contains positive elements, and implies a deeper contingent-pragmatist structure. To his credit, Rorty recognises this in identifying the problem of ironist theory being "how to overcome authority without claiming authority", his answer being to eschew theory altogether. This reply does not sit easily alongside a body of work that is as much theoretical and metaphysical as it is personal. ${ }^{63}$

\footnotetext{
${ }^{63}$ Ibid, pp105, 108.
} 


\subsection{Justification and liberalism}

Regardless of this problem with Rorty's underlying structure of contingency, his justification of a radically uncertain conception of liberalism remains interesting and important. According to his idea of the contingency of language and the liberal tradition, his approach amounts to a non-foundational redescription of liberalism. This means, he argues, that there is no non-circular or final justification of liberal solidarity, and that liberal democracy is not in need of a philosophical justification at all. ${ }^{64}$ This view this leaves Rorty with a problem, as expressed by Anthony Langlois:

[I]f Rorty is to be taken seriously when he says that there is no reality beyond language... there is no reason why we should prefer Rorty's story telling method of promoting the human rights culture over the reasoned exegesis of the foundationalist philosopher. Both can be said to be arguing for the same thing but using a different final vocabulary, and which one you opt for is no more than your personal preference. ${ }^{65}$

This is an attack on Rorty's framework of contingency, and, in particular, on the hidden assertion that a contingent viewpoint is the best approach in a contingent world. It implies that theory, despite some sense of conflict with Rorty's view of the underlying 'reality' of contingency, might actually have an important role in society. This role would be expressed by its use in building an understanding of reality based on theoretical conventions and ideas.

In relation to liberalism, Langlois argues that, without justification, there is no particular reason, beyond that of mere preference, to accept Rorty's irony-solidarity conception. Rorty would probably largely agree with this, but would note that this preference is guided by all the moral custom and liberal political tradition of western beliefs. This would mean that the general preference for such a redescription of liberalism would have real moral force, and even truth, to it. Rorty's justificatory burden, then, involves explaining why his redescription of liberalism is preferable to other vocabularies and descriptions, particularly those that involve a liberalism that is foundational and theoretical. This project can only be achieved, Rorty argues, from

\footnotetext{
${ }^{64}$ Rorty, op cit, above note 2, pxv; Rorty, op cit, above note 11, pp178-179.

${ }^{65}$ A J Langlois, 'Redescribing Human Rights' (1998) 27(1) Millennium: Journal of International Studies 1, p9.
} 
the perspective of the particular tradition in question, namely the institutions, practice and thought of western liberalism. His project, then, is to justify his liberalism within this tradition. To achieve this he can invoke those western and liberal beliefs that he considers useful. Rorty, however, in justifying his liberalism, also seems to adopt a conception of humanity and solidarity that reaches beyond the beliefs of liberalism. The goal is to present a redescription that is accepted by virtue of it allowing people to better interact with their environment, meaning that it results in a better-ordered, happier society.

That Rorty begins with a negative conception of contingency, that is radically open and does not discern between constructed truths, and ends up with a vision of liberal utopia, is surprising. Honglim Ryu recognises this in commenting that most postmodern thinkers believe ethics to be doomed: ${ }^{66}$

The postmodern approach to ethics does not provide any determinate framework for deciding how to adjudicate conflicting ethical claims or how to link ethical unconditional affirmation of the emancipatory ideals, enlightened social criticism, and democratic accountability in determinate political terms. ${ }^{67}$

Ryu argues that the conditions of postmodernism, in Rorty's case of contingency, do not offer adequate means for justifying liberalism as being any more or less valuable than another political ideal. Ryu goes on to conclude that the ultimate problem with postmodern thinking is that it "cannot overcome and deconstruct itself because it does not specify its own historical and social background". ${ }^{68}$ This seems to mean that postmodernism fails because it understands itself only in absolute and universal terms. As is suggested, postmodernism can never criticise itself on the same terms that it uses to criticise more obviously universalist theory. If contingency is seen as merely a product of a particular historical and social context, then it loses its power of critique against opposing beliefs.

Ryu argues that this removes any possible framework for ethical judgment, and, so, undermines Rorty's attempts at presenting a valid conception of liberalism. Norman Geras agrees with this conclusion. In his book, Solidarity in the Conversation of

\footnotetext{
${ }^{66}$ H Ryu, 'Ethics of Ambiguity and Irony: Jacques Derrida and Richard Rorty' (2001) 24 Human Studies 5, p8.

${ }^{67}$ Ibid, p7.

${ }^{68}$ Ibid, p25.
} 
Humankind: The Ungroundable Liberalism of Richard Rorty (1995), he argues that, with no truth, there is no justice. ${ }^{69}$ If there is no correspondence with reality, then redescription can achieve virtually anything.

[I]f we take him at his word on the subject of incommensurable vocabularies or language games, of anything being able to be made to look good or bad by means of a redescription, it would seem to follow that we lack non-arbitrary ground for distinguishing between better views and worse ones. ${ }^{70}$

Ryu and Geras agree that, if contingency is to be taken seriously then there is no perspective from which to view ethics. Rorty's response to this might again be to concede the point, and confirm that he is not interested in attempting a non-circular or transcultural justification. However, these criticisms seem to go further, in that they question the approach that Rorty actually does take: to look for agreement within the context of a society or tradition, and to appeal to the standard of pragmatism.

The first of these might be criticised on the grounds that the ideal of agreement within a tradition is an external and arbitrary standard, and, so, conveys no moral value in the context of a contingent reality. Rorty might reply that, since morality itself is made up of the customs and beliefs of that tradition, using agreement as a moral criterion amounts to applying a standard already part of the nature of morality. In this way, the general agreement to a moral redescription can act as the benchmark condition for an improvement in moral value. Examining the nature of this improvement requires looking at the use of pragmatism as part of the redescription's justification.

It is interesting that Rorty perceives morality in terms of a unity of agreement. Like most postmodernists, he strongly supports social criticism, but he sees the proper and natural end of such criticism to be the replacement of the current vocabulary implied by general agreement. Fellow postmodern thinker Steven Hendley has objected sternly to the tendency in Rorty's work to assume the importance of a singular expression of morality and liberalism. Hendley argues that,

[o]nce we are unable to take that universal identification with humanity for granted, we have lost the basis for taking ourselves for granted in any sense, no matter how finite. The issue of

\footnotetext{
${ }^{69}$ N Geras, Solidarity in the Conversation of Humankind: The Ungroundable Liberalism of Richard Rorty (1995), p107.

${ }^{70}$ Ibid, p123.
} 
who we are becomes subject to question in terms of multiple voices that may no longer be assumed to share a common interpretive horizon. ${ }^{71}$

This leads Hendley to conclude that we should think of democracy as "an inherently multiple and contested process", ${ }^{72}$ In other words, it is wrong for Rorty to conceive of the public justification of a moral redescription simply in terms of replacing one agreement on a set of principles with another; such a view obscures the plurality of voices within a society. Rorty might respond that he is talking of redescriptions within a particular group or tradition, rather than a society, but this is not sufficient in that he gives no account of how a plurality of groups and group beliefs are to be accommodated within a society, and he offers no account of the diversity internal to a group or tradition. Normally, a postmodern thinker would be expected to champion such diversity of belief, but this seems to be absent in Rorty's account of agreement.

The other aspect of the justification of redescriptions concerns the criterion of pragmatism. According to Rorty, individuals and groups determine contingent moral content in order that they should interact with their environment in the most effective way. Geras argues that this standard is defective in two ways:

First... there is a plurality of different purposes against which to estimate whether or not any theory, belief or language game helps us to cope. Second, even for some one given purpose, it would not be credible in view of the rest of what he thinks for him to offer coping in or toward that purpose as a logically basic, would-be objective datum. What counts as coping or working, as instrumental success, must be also for its part subject to interpretation, to beliefladen, language-governed understanding. ${ }^{73}$

Geras argues here that the idea of pragmatism, of interacting with one's environment in the most useful way, is too general to be given any substantive moral direction: it offers no direction on what a particular belief must be useful for, and how its usefulness is to be understood and interpreted.

Either, Rorty would need to set out a more specific set of rules by which his conception of pragmatism could be usefully applied, or, individuals would have to inform the pragmatist approach with their own existing beliefs. The former is unattractive in that it would make it clear that there are substantive external (and

\footnotetext{
${ }^{71}$ S Hendley, 'Putting ourselves up for question: A postmodern critique of Richard Rorty's postmodern bourgeois liberalism' (1995) 29 The Journal of Value Inquiry 241, p247.

${ }^{72}$ Ibid, p251.

${ }^{73}$ Geras, op cit, above note 69, p123.
} 
universal) principles being applied in the justification of redescriptions. The latter is equally problematic in that it means that the operation of pragmatism itself is contingent. This goes against Rorty's manifest intention that pragmatism is to ground and direct the use and evolution of contingent beliefs. Rorty seems in a bind here. $\mathrm{He}$ attempts to apply a broad, generally acceptable, idea in pragmatism, but it seems either to be a universal, external value applied to justify beliefs, or so general to have no meaning on its own. Generally, some form of justification of Rorty's nonfoundational redescription of liberalism is necessary. However, his seems to incorporate potentially contestable conceptions: not just of contingency generally, but of the societal agreement on pragmatic grounds that implicitly underlies the redescription. The latter seems strangely singular and unable to be determined in a meaningful way, so that, even if the contingency of liberalism were accepted, the value of any particular redescription would be unknowable.

This analysis of Rorty's approach to liberal justification begins to reveal a tension between his two most fundamental conceptions, namely contingency and pragmatism. With contingency, all beliefs are simply linguistic expressions that attach to other nodes in the web of beliefs and desires that comprise our selves. With contingency alone, Rorty could only conclude that any assertion of moral truth or value is flawed and that there is no criterion by which to choose and judge a belief other than from the perspective of our existing beliefs and desires. Contingency, viewed at the most fundamental level, appears to be self-refuting, in that its universal and absolute consequences belie its own content. Pragmatism grounds contingency in our particular environment, and offers a positive understanding of the way we construct our beliefs. It offers a reason why people might choose a redescription of moral principles over the previously agreed description. Rorty uses this reasoning in order to justify particular redescriptions. This seems to require, either, that pragmatism be given more concrete meaning, which is contrary to contingency, or, that it becomes contingent itself and, so, offers no more direction than is offered by contingency. Within Rorty's theory there is a curious tension between the postmodern ideal expressed by contingency, and the desire for a positive, agreed mode of a liberalism that is more than just accidental and arbitrarily justifiable. In the abstract sense, at least, it seems that pragmatism cannot easily fill this role. 


\subsection{Ironism and solidarity}

The final step in the analysis of Rorty's postmodern project is to examine his conception of liberalism and its justification, with particular reference to the way that Rorty employs pragmatism as a justificatory device. His conception consists in the relationship between self-creation as ironism, and solidarity as the liberal avoidance of cruelty. This relationship is one of equality and incommensurability, and corresponds with the division between the private and the public. As has been evidenced by earlier chapters, most liberal theories tend to define a space within which individuals are free to determine their own way of living. For Rorty this space consists of the personal domain, and the nature of the freedom afforded corresponds with his conception of ironic self-creation. This approach involves the awareness of contingency, even in relation to one's own beliefs and vocabularies, yet promotes the exercise of autonomy in choosing one's own conception of how to live, and challenges the rules inherited from a group, culture or tradition.

The most powerful criticism of this ideal comes from Honi Haber, who focuses on the fact that, as Rorty admits, this ironism would be the exclusive domain of the intellectual minority who are able to recreate themselves in this way. ${ }^{74}$ This would mean, she argues, the institutionalisation of the intellectual elite, and would have a number of disturbing consequences:

Since the public rhetoric cannot be ironist, the youth will have to be brainwashed, the public sphere founded upon noble lies. At the level of the public, grand narratives are legitimate even if false - for irony is 'inherently a private matter', a skill taught only in the school of the privileged elite. $^{75}$

The public consequences of this private elitism, Haber argues, are far from being liberal, and actually reflect Rorty's personal view of intellectual self-creation. This portrayal of Rorty's position is not entirely fair. He does admit that, in a liberal state, non-intellectuals would never be able to be fully-fledged ironists. Such people would,

\footnotetext{
${ }^{74}$ H Haber, 'Richard Rorty's failed politics' (1993) 7(1) Social Epistemology 61, p66.

${ }^{75}$ Ibid, p67.
} 
however, intuitively adhere to some basic ironist beliefs: that social and historical context is always relevant, and that there is no intrinsic essence to belief. ${ }^{76}$

This does not defend entirely the claim of elitism, and it raises the question of why ironism is the proper focus for a conception of personal freedom. Even if the contingency of reality were accepted, it does not automatically follow that personal freedom should mean ironic self-creation. A better conception might be of personal freedom as traditional freedom, which would place the focus on choosing from within the boundaries anticipated by the history and practices of society. In western society these boundaries would already be broad, and admit plural and highly varied approaches to life. This conception would not exclude radical ironism anyway; it would simply place such ironism on the periphery of the concerns of freedom, rather than at the centre. Ultimately, the power of ironic self-creation depends on the necessarily central importance of ironism.

The second part of Rorty's liberalism is solidarity, as the liberal avoidance of cruelty and humiliation. Rorty's idea of solidarity as the central concern of the public domain is itself troublesome. The nature of the trouble is that the universality with which he views solidarity is seemingly at odds with the most basic ideas of contingency, and, in particular, with the lack of any conception of a common human nature. Rorty's basis for this is the "claim that we have a moral obligation to feel a sense of solidarity with all other human beings" ${ }^{77}$ This is not founded upon "the recognition of a core self, the human essence, in all human beings", but, rather, as "the ability to see more and more traditional differences... as unimportant when compared with similarities with respect to pain and humiliation". ${ }^{78}$ So, Rorty's account of solidarity depends on being able to effectively argue that human nature is contingent and constructed, whereas human pain and suffering is universal. This is the basis on which morality is mere custom, but solidarity is of general human concern. This is exemplified by his claim that

you cannot aim at 'doing what is right', because you will never know whether you have hit the mark. Long after you are dead, better informed and more sophisticated people may judge your action to have been a tragic mistake... But you can aim at ever more sensitivity to pain, and

\footnotetext{
${ }^{76}$ Rorty, op cit, above note 2 , p87.

${ }^{77}$ Ibid, p190.

${ }^{78}$ Ibid, p192.
} 
even greater satisfaction of ever more various needs. Pragmatists think that the idea of something nonhuman luring human beings on should be replaced with the idea of getting more and more human beings into our community - of taking the needs and interests and views of more and more diverse human beings into account. ${ }^{79}$

For Rorty, the proper goal of moral custom is to be inclusive of various diverse people, to work to diminish the pain suffered by the included, and to satisfy their needs and desires as much as is possible. This claim, he implies, is not a moral or customary claim, but is beyond and should direct morality. It is a broadly pragmatic expression of how morality can promote the most effective interaction between people and their environment.

Geras attacks this account of solidarity by invoking the tension between Rorty's rejection of human nature and his universal conception of human pain:

Is there not something rather implausible about insisting on the communal sources of strong solidarity, and insisting at the same time on the irrelevance of the idea of a common humanity to the goal of more expansive solidaristic relations $?^{80}$

In particular, Geras criticises Rorty's account of us as coreless and contingent selves and, then, Rorty's retraction of this account when he wants to draw some universal moral conclusions. This account, he claims, entails that

we are asked to believe... that solidarity nourishes itself, receives its strength and vigour, from commonalities which are concrete, palpable, lived; and asked also to believe... that feelings of extensive mutual sympathy can be created amongst beings who, of almost infinite plasticity, may share with very large numbers of other such beings no common features at all. ${ }^{81}$

Here Geras explicitly sets Rorty's idea of the radical plasticity of the coreless self against the uniformity of pain, and the feelings of solidarity that this uniformity is supposed to be able to engender.

Rorty's account of solidarity is certainly ingenious: at its centre, solidarity is not moral in nature, but pragmatic. It comes not out of our moral intuitions, but, rather, the idea that the interaction of people with their environment is most successful when public action is taken according to rules that take the pain, desires and needs of others into account. On Rorty's account, morality is the customary expression of this

\footnotetext{
${ }^{79}$ Rorty, op cit, above note $15, \mathrm{p} 82$.

${ }^{80}$ Geras, op cit, above note 69 , p90.

${ }^{81}$ Ibid, pp90-91.
} 
principle, and philosophy may assist in effectively describing it; but, the pragmatic foundation of solidarity is beyond both of these social narratives. ${ }^{82}$

The assertion that there is substantial pragmatic value to solidarity, such that the latter should direct a society's public sphere, is extremely potent, and relies on the fundamental nature of pain and humiliation on one side, and the satisfaction of needs and desires on the other. But, it is far from being straightforward and unambiguous. The ideal of pragmatism is described above as being unworkably broad. Solidarity does represent a narrowing of this, but the result remains quite broad. The idea of inclusion is relatively definite, but the manner of reducing pain and increasing satisfaction is not determined. Presumably, these details would be determined by the particular vocabulary and description of solidarity constructed within a society, and the corresponding moral principles.

There is certainly a general attraction to this conception of pragmatism, although Rorty's approach suggests that we should be wary of this attraction since it is probably based on our customary moral intuitions. What, instead, is important is the necessary link between pragmatism in the public sphere and solidarity. The principle of inclusion does not seem to be clearly derived from pragmatism in the required way, in that an approach based on the concern for people in groups could be pragmatically superior depending on social or historical conditions, or existing political institutions or beliefs. Perhaps this inclusion depends on the conditions of modern western societies, namely the existing technologies available to public and political action, and the internal individual and group diversity.

If inclusion shifts from being a universal aspect of solidarity, then what remains is the avoidance of pain and the promotion of satisfaction. Policies that best express these ideals, Rorty argues, allow people to interact most successfully with their environments. On such broad terms, one cannot help but agree, except perhaps to recognise that this implies a collectivist perspective that is not inherent in the pragmatism itself. It still seems hopelessly broad, however, and it is difficult to see how it could be used to justify positively one liberal description over another. Perhaps

\footnotetext{
${ }^{82}$ Rorty, op cit, above note 2, p196.
} 
this is Rorty's point in saying that liberalism has no foundations and no non-circular justification, but, then, solidarity offers no reason to redescribe. Either, Rorty's solidarity is a positive foundation for his description of liberalism, or, it has no role whatsoever in influencing social meaning; either, pain and suffering are understandable across individual and group contexts, or, they have whatever meaning a group gives to them. If there are no foundations and no non-circular justification to liberalism, then Rorty's liberal ironism has no value in relation to solidarity.

It is this lack of cohesion between solidarity and liberalism that is the greatest problem in Rorty's liberal conception. He wants to use the general and pragmatic value of solidarity, but at the same time, keep liberalism contingent and untethered; however, he cannot quite manage to achieve both at once. The direction to give up "the philosophical search for commonality", while, at the same time, "noticing the similarities" between ourselves and others, turns out to involve too much of a contradiction. ${ }^{83}$ It is this contradiction that underlies the observations of Geras, and that expresses further the tension between contingency and pragmatism in Rorty's work.

\subsection{Liberal ironism}

Despite this, Rorty's particular redescription of liberalism is of interest, as what purports to be the best western expression of political solidarity. Rorty's adoption of Shklar's avoidance of cruelty formula has drawn considerable critical comment. It is clear that there are alternative, competing conceptions of liberalism, and that Rorty has accepted one as representing the best available description that accords with his central commitments to accepting contingency and pragmatism. This assertion has been criticised in a number of ways.

Haber, for example, argues that the ideas of cruelty and humiliation are too broad, and omit much of what is actually moral.

\footnotetext{
${ }^{83}$ Rorty, op cit, above note 15, p86; Rorty, op cit, above note 2, p196.
} 
Universal concepts, be they rationality or humiliation, have force only so long as what marks us out as being the individuals we are is ignored. They are all, I would argue, politically dangerous concepts in that their supposed neutrality always masks a particular, non-universal, particular agenda. This is clear in the case of Rorty who blithely defends the institutions of western democracies, even they entail oppression, on the grounds that such institutions lesson $[$ sic $]$ cruelty and suffering. This is, of course, a naïve view. Whether or not it lessens cruelty and suffering is a matter of how those terms are conceived, and if ironism and postmodernism politics has anything to teach us it is the infinite variety of such narrative constructions. ${ }^{84}$

Haber criticises firstly the generality of cruelty and humiliation, in that by themselves, these are undefined and potentially obscure a more particular approach. Secondly, she infers the poverty of these ideas as expressing the whole of a theory of justice, and that it excludes such moral concerns as oppression. Both of these arguments carry some weight. Cruelty and humiliation, as used by Rorty, seem to possess little clear content. It does seem that he would want to give them something of their normal emotional meaning, and, so, distinguish them from other negative moral states such as oppression. In picking up on the latter point, Charles Anderson describes cruelty as an "extreme and spectacular case", and argues that liberals must also be concerned with "the more workaday and prosaic virtues, with fair dealing and simple justice, with arbitrary and capricious authority wherever it exists". ${ }^{85}$ It seems that Rorty is concerned with acts that are a deliberate affront to individuals' interests and selfconceptions, and that amount to abuses of power. These may be, as he claims, the worst things that we can do; but what these are supposed to mean, whether other values are promoted by liberalism, and what institutions are likely to result, are unclear from Rorty's text.

The final point about Rorty's account of justice concerns the marriage of liberalism and ironism into a single conception. He conceives of liberalism and ironism as essential and incommensurable parts of the well-lived, moral life, and that they should have ascendancy in the public and private spheres respectively. The separation of a sphere that is open and expresses liberal freedom, from a sphere that expresses the commitment to liberalism, is a recurrent strategy in liberal theory. It has the effect of preventing the openness of liberalism from undermining the liberal message itself. Rawls' conception of the priority of the right over the good, and, later, of the separation between public and private, are the best examples of this.

\footnotetext{
${ }^{84}$ Haber, op cit, above note 74, p72.

${ }^{85} \mathrm{C}$ W Anderson, 'Pragmatism and Liberalism, Rationalism and Irrationalism: A Response to Richard Rorty’ (1991) 23(3) Polity 257, p370.
} 
Both of these approaches are flawed. The separation of the right and the good, and the characterisation of the former as being moral, necessary and singular, and the latter as personal, contingent and plural, is doubtful, and brings into question much of the project of A Theory of Justice. The public/private division in Political Liberalism is more effective in that it does not attempt to make a fundamental distinction about the nature of human life and endeavour, but, rather, is supposed only to define a liberalism that is acceptable to those who are reasonable. This is an interesting and pragmatic approach that is criticised above for drawing too clear a distinction between the two, and for presenting the public realm as being too unified. ${ }^{86}$

Rorty's distinction between the public and the private, or between self-creation and liberalism, is also pragmatic, but in a different sense than that of Rawls': it is supposed to reflect a redescription of human life that engenders the best and most effective lives. The standard here is not the acceptability of the resulting system to any person or group, but, rather, its pragmatic value. The earlier criticisms of this type of liberal approach suggest that this distinction should be closely examined to determine whether it can actually perform the role to which it has been assigned. This is reflected in the comment of Paul Forster:

To say that public obligations have no automatic no necessary priority over private aspirations is not to say, as Rorty does, that they are incommensurable in principle, that they are necessarily opposed kinds of goods. Nor is it to say that there must always be a sharp distinction between a private ethic of self-creation and a public ethic of mutual accommodation. It is only by confusing the dichotomy between the public and the private with the philosophical distinction between the contingent and the necessary that Rorty can suggest that pragmatists are committed to the political thesis that conceptions of the good life have no place in politics and are to be consigned to private life. ${ }^{87}$

The idea of the contingent and the necessary represents well Rawls' distinction between the good and the right. Forster is arguing that, with his conception of the public and private spheres, Rorty perpetrates the same error, in that he draws too sharp a distinction between what is personal and non-moral on one hand, and what is moral and universal on the other; in other words, that the dualism of self-creation and liberal solidarity does not fully mirror the dualism of private and public.

\footnotetext{
${ }^{86}$ Above, chapter 6, note 120.

${ }^{87}$ P D Forster, 'Problems with Rorty's Pragmatist Defence of Liberalism' (2000) 25 Journal of Philosophical Research 345, p355.
} 
This is reflected in Anderson's criticism of the separation of public and private, and with it, "the separation of the spheres of rational criteria and subjective expression, knowledge and opinion, public responsibility and personal preference". ${ }^{88}$ The absolute nature of this separation, Anderson argues, means that Rorty ignores the more naturally pragmatic position, that all action is intrinsically public. ${ }^{89}$ This "deprives life of most of its meaning", by excluding the collaborative and common aspects of our personal lives. ${ }^{90}$ This does seem an effective indictment of Rorty's limitation of self-creation to the private world. Haber sees this as reflecting "an unexamined, inherited understanding of human nature as competitive and self interested". ${ }^{91}$ It is this conception that informs the need to limit ironic self-creation to the private sphere, as its influence on politics would be cruel and destructive. ${ }^{92}$

Both of these accounts suggest that Rorty's separation of the public and private suffers from the same problems as does Rawls': that the structural separation implied is belied by the reality of those conceptions that are sought to be distinguished. Each of these distinctions informs a structure that allows liberalism to function. For this reason, it seems that Rorty's cleaving between public and private does not match, in the meaningful way implied, the cleaving between self-creation and solidarity, and this undermines the conception of liberalism as expressing each in a way that reflects their equal status and the incommensurability between them.

\section{Conclusion}

Like the liberal developments in the areas of pluralism, political liberalism, public reason and multiculturalism, Rorty's postmodernism implies a position that is sensitive to the reasonable contestability of ideas. It is not concerned with the diversity of beliefs across a population, but, rather, the nature, substance and power of beliefs. Rorty asserts that beliefs are contingent, and not representative of reality.

\footnotetext{
${ }^{88}$ Anderson, op cit, above note 85, p367; see also Topper, op cit, above note 4, p761.

${ }^{89}$ Anderson, op cit, above note 85, p367.

${ }^{90}$ Ibid, p369.

${ }_{91}^{91}$ Haber, op cit, above note 74, pp64-65.

${ }^{92}$ Ibid, p65.
} 
As was suggested in the introductory chapter, an approach that expresses radical moral uncertainty has two problems: firstly, that the assertion of non-absoluteness is itself absolute and, so, is self refuting; and, secondly, that such uncertainty resists the distillation of our diverse circumstances and beliefs into a unified moral and political system. The first of these can be levelled squarely against Rorty's conception of contingency. This asserts the radical contextual importance of our appreciation of reality, on theory, and on our desires and beliefs. It also means that there is no truth in the sense of representing the real or actual situation, and only that which is constructed out of our language, and used as a tool to enable use to deal better with our environment. It is difficult to see how Rorty can make the assertion of contingency without this itself being a constructed and non-foundational idea. The latter position is logically flawed, however, in that accepting the contingency of contingency implies a higher level, correct understanding of contingency. In addition, if contingency is itself contingent, there is no reason, intrinsic to contingency itself, for adopting it.

Rorty attempts to overcome the second difficulty with a conception of pragmatism. This move tethers our beliefs to our environment according to a general ideal of allowing us to live well. There are two problems with such an approach: firstly, there is a tension between this tethering of our beliefs and our environment, and the idea that contingency means the non-representation of reality; and, secondly, that pragmatism as a criterion is too broad to have any useful meaning. In relation to the former problem, it seems that pragmatism might act to augment and strengthen the idea of contingency, so that beliefs would still be non-representational, but they would at least help us to live in the manner of an evolutionary development. ${ }^{93}$ In this way, our beliefs would be useful in relation to our environment, even if they could not represent the latter. The problem with this view is that pragmatism implies a relationship to our environment that contingency denies. In relation to contingency, for example, truth is remade into the expression of intersubjective agreement. This is inconsistent with the pragmatic view of truth as being those beliefs that allow us to interact with our environment in the most advantageous way.

\footnotetext{
${ }^{93}$ C.f. Rorty, op cit, above note 15 , p65.
} 
The second problem with pragmatism is that it is not itself sufficiently meaningful to direct our understandings or our actions. In a broad sense, it is possible to see that having a belief system has general benefit, in allowing complex social structures. It is possible to see the benefits of a liberal system on this level; as being better for individuals than a non-liberal, authoritarian or state-of-nature society. ${ }^{94}$ This analysis, however, is insufficient to justify a particular conception of liberalism, or liberalism as against non-liberal or quasi-liberal systems that feature many of the same general benefits. In this sort of comparison, the general idea of pragmatism is too broad and too uncertain as a potential source of justification.

It is true that this does not quite accord with Rorty's own approach of redescription, whereby some beliefs and parts of vocabulary are reformed so as to improve an existing set of beliefs. In this situation, we would have our foundational and other beliefs to direct our judgment of a potential redescription, and, so, to inform the application of our pragmatism. The problem with this approach is that, by basing pragmatism on our existing beliefs, it begins to lose its meaning; it becomes embedded in our contingent viewpoint so that it promotes changes that allow us to live better in relation to our existing beliefs. This approach is a coherence conception of pragmatism, which presupposes that those beliefs that will be preferred will be those that cohere with our other beliefs. As such, it undermines the more general Darwinian view of pragmatism that Rorty asserts. ${ }^{95}$

This would make it difficult to endorse Rorty's conception of justice as being the equal promotion of self-creation as ironism, and of solidarity as the liberal avoidance of cruelty and humiliation. Both of these conceptions carry some value, yet are narrow in their meaning: self-creation, and especially ironic self-creation, as an expression of personal freedom; and the avoidance of cruelty and suffering as an expression of solidarity. The more general difficulties with his theory leave little ground on which to justify these ideas, and there is no reason to think that they represent a favourable redescription of liberalism, or of justice. As many have commented, they seem more an expression of Rorty's personal beliefs; the idea that

\footnotetext{
${ }^{94}$ C.f. above note 50.

${ }^{95}$ Rorty, op cit, above note 15 , p269.
} 
we would agree to them reflects his limited conception of what 'we', as citizens of western democracies, means. ${ }^{96}$

This failure is unfortunate, as the idea of a radical contingency is quite compelling as an expression of moral uncertainty. Perhaps Rorty would do better to begin, not, from a universal theory of contingency, but, from contingency as a social fact, in other words, to work from the inside out. This would mean that the general theory would be the kind of non-foundational theory that Rorty suggests liberalism should be. This would make contingency, not a theory of reality and truth, but, rather, a theoretical representation of the social existence of doubt. With this approach, contingency ceases to be foundational, and takes on the central importance of a socially fundamental fact, as reasonable pluralism does for political liberalism, and persistent disagreement does for deliberative democracy. This would mean that contingency itself is not a truth, but is a reflection of how things seem to be in western societies. This entails a more tentative approach than Rorty allows, one more akin to doubt about the possibility of absolute truth, than to disbelief.

Such an approach would overcome the argument that contingency is self-refuting, in that the assertion of contingency does not itself claim to be absolute. It would also allow the possibility of moral and liberal theory that would be justified by the interplay of socially accepted norms and criticisms, as well as textual and theoretical descriptions of these. This would, most likely, lead to a liberalism that is more openly conceived and more dialogical than Rorty allows. This approach would face two initial criticisms: that the assertion of contingency, by being non-foundational, represents a contestable view of reality, and offers no reason for its own acceptance; and, that such an approach is not prescriptive in any way, but, rather, describes the process by which moral beliefs and political practices change.

Overall, Rorty's project of developing a justification of a conception of liberalism that is successful, despite being non-theoretical and non-foundational, fails, in a way that corresponds to the second difficulty with sceptical theories, that of finding agreement in the context of uncertainty. To the extent that it draws substantive content from

\footnotetext{
${ }^{96}$ E.g. Haber, op cit, above note 74, p71.
} 
pragmatism, it becomes foundational and universal; and, to the extent that it does not, it can be justified only by the agreement of western liberal citizens, who might as readily subscribe to some other liberal or political ideal. The possible reply, that such people should accept the contingent nature of reality as a fundamental part of their belief systems, is not justified by the idea of contingency itself. 


\section{FREEDOM AND UNCERTAINTY}

\section{Contemporary liberal theory}

\subsection{Approach of thesis}

The aim of this thesis is to examine contemporary liberal theory from the perspective of moral uncertainty. In order to explicate the requirements of this perspective, the introduction considered three significant approaches to moral theory that embody some form of moral scepticism or doubt. The ideas of Protagoras, Bertrand Russell and Jean-François Lyotard offer various expressions of this moral uncertainty. Despite the cursory way in which their different understandings of moral theory are presented, it is clear from them that moral uncertainty is a complex moral position that cannot be defined by one proposition or principle. The general themes that are taken from the work of these three philosophers are: firstly, a doubt as to the possibility of universally justifiable moral theories; secondly, the contestability of alternative and conflicting moral judgments, which the application of rational analysis cannot solve; thirdly, the inherent complexity of moral positions, which resists attempts to be reduced to a meaningful theoretical structure; and, finally, the contingency of moral positions, meaning their dependence on their socio-historical context, and on the personal beliefs of particular theorists.

The approach of examining liberal theories from the perspective of this uncertainty requires that they are analysed in order to discern whether and how these particular arguments apply. In this way, the theories are implicitly viewed as responses to moral uncertainty; as attempts to accommodate or overcome these arguments. The approach I have often taken is to set out the justifications and structures of particular theories in their entirety, before examining them critically. This has the virtues of allowing the theories to be viewed as potentially coherent wholes, of allowing complex arguments that are relevant to multiple aspects of the theory to be presented, and of initially 
taking a theory on its own terms. The idea here is that this process involves expressing and, in a sense, building a theory, so as to best reveal its strengths and weaknesses. In performing this task, the initial analysis focuses on the universal aims and justificatory strategy of particular theories, but also covers their substantive processes and prescriptive principles and institutions. The latter substantive aspect cannot be excluded from this analysis; although the aims and justifications of these theories are more directly relevant to issues of moral uncertainty, their substance and prescription are expressions of these justifications from which they cannot be meaningfully separated. They are also the locations of many of the problems of these theories, relative to moral uncertainty.

Many contemporary liberal theories have been canvassed using this method. These theories, and the approach the thesis takes to organise them, fall into two separate periods, which could be labelled as early and post-communitarian. Early contemporary liberal theory is the subject of the first three substantive chapters. It includes Rawls' A Theory of Justice, Nozick's libertarian response in Anarchy, State, and Utopia, and the more egalitarian work that followed, from Dworkin and Ackerman. Here, the work of each thinker is considered in turn. For Rawls, Nozick and Ackerman, this task is structurally straightforward, as each has a single work that presents their conception of liberalism. In contrast, Dworkin's theory is scattered among a number of essays. These have been approached chronologically, and some of the development in his thought has been considered.

The post-communitarian period of contemporary liberal theory occurs later than the earlier period, beginning in the early-mid 1980s. This period is characterised by a shift in approach away from the earlier works: from the construction of liberal structures by hypothetical situations that pare away morally irrelevant or arbitrary details, to the examination of the nature of difference and belief within western societies. These changes reflect doubts about the earlier approach, many of which were prompted by the communitarian criticisms that were central to liberal political theory during the 1980s. These later theories are organised into groups of particular approaches. They cover conceptions of liberalism that hold various ideals or principles to be liberalism's central concern, and deal with, pluralism, political liberalism, public reason, multiculturalism, and postmodernism. 
The final task of this thesis, addressed in these concluding remarks, is to draw together the points learned about how various contemporary liberal theories respond to moral uncertainty, to find those approaches that represent potentially valuable liberal conceptions, and to anticipate possible future shifts that would achieve and develop this potential.

\subsection{Early contemporary liberal theory}

Rawls, in A Theory of Justice, shows considerable awareness of the challenges of moral uncertainty. This is suggested by the limitations imposed on its justificatory process, namely that his conception of justice as fairness is compared with a number of potential alternatives, rather than being presented as the best conception if justice. It is most obvious in relation to the method of justification itself. Rawls' theory does not derive his two principles directly from human nature, or from any absolute conception of reality, but, rather, from the considered intuitions of his readers. The latter are supposed to consider the conditions of the original position and the two principles that result, and to find an equilibrium between their moral judgments of both.

This idea holds considerable attraction, in the way it works from our own moral beliefs, yet attempts to meld these with a carefully structured theoretical system. This approach is engaging enough to overcome the criticism that intuitive beliefs do not accurately reflect objective moral value, and, so, cannot achieve the view from eternity for which he aims. Closer examination of the structure of the theory, however, reveals that Rawls' reliance on reasonable and rational judgment is considerable, and means that, while Rawls has presented a plausible conception of justice, he has not acknowledged the plausibility of alternative positions and ideals. This is most obvious in relation to the derivation of the difference principle from the original position: commentators who are not as egalitarian as Rawls consistently show how other principles of distributive justice could be reached by those in the original position. 
Another form of this plausible, but contestable, content concerns the conceptions of the person and of goods that are implied by the original position. These serve to inform Rawls' theory with individualistic, humanistic and recognisably liberal meaning that is presupposed rather than justified. The presence of these forms of contestable content serves to undermine Rawls' approach in that it implies a bias that undermines his justificatory aims.

The organising principle that structures much of the content in A Theory is the priority of the right over the good. In order to justify this relationship, Rawls employs a contrast between liberty and right on one hand, and the desirability of increasing aggregate welfare on the other. ${ }^{1}$ This account oversimplifies and obscures our moral lives: its characterisation of the right as liberty and morality, and as being singular, universal, and rationally appreciable; and, its characterisation of the good as welfare and personally held conceptions of how to live, and as being diverse and morally secondary, is flawed. The weakness of this priority brings into question much of Rawls' theory, from the exclusion of the knowledge of one's conception of the good from the original position, to the priority of the liberty over the difference principle. $A$ Theory of Justice is a theoretically ingenious attempt at justifying a liberal conception of justice, and its position at the forefront of recent liberal theory is undeniable. Despite this, its project is undermined by its plausible, but contested, content, and by the flawed conception of the right and the good.

Anarchy, State, and Utopia is known as Nozick's libertarian response to Rawls' egalitarianism, but the two share many structural elements. The most obvious is the hypothetical narrative Nozick employs to get from the state of nature to the minimal state, but not further. This approach, however, uses a legitimacy standard that expresses an approach quite different from that of Rawls. The natural rights that Nozick employs are not justified by reference to people's considered moral judgments, but, rather, Nozick declares that they are universal and cannot be abrogated. He imagines that they stem from what makes humans lives morally meaningful, but his justificatory explanation of this is wholly inadequate. For this

\footnotetext{
${ }^{1}$ See above, chapter 2 , note 66.
} 
reason, his theory fails to vindicate these rights as being morally incontestable, and ignores many of the potential complexities that exist in the moral sphere in which the rights operate. Despite the deftness with which Nozick builds the minimal state from the state of nature without breaching these natural rights, the system is undermined by the legitimacy of the rights themselves.

A comparison of the approaches of Nozick and Rawls is a potentially fertile exercise. Although the two reach disparate distributional outcomes, their approaches have much in common. Both are based on deontological premises, so that each defines a set of rights that are morally prior to considerations of welfare. For Rawls, this is achieved through the original position, but, ultimately, this mirrors the priority of the right over the good. Nozick's natural rights define this moral priority. Both of these approaches are plausible, and appeal to particular intuitions. However, the two represent clearly different liberal ideals, and this difference itself suggests a rational contestability that neither theory answers adequately.

The work of both Dworkin and Ackerman represents a return to egalitarian conceptions of liberalism, although both take different approaches to that of Rawls. Dworkin identifies the core liberal value as being an equal concern and respect for all people. He argues that such equality should be as neutral between particular conceptions of the good as possible. He justifies this conception primarily by suggesting that it satisfies those features that we would expect would attach to the legitimate liberal core, namely that: it represents authentic liberal intuitions; it is sufficiently compete; it is distinct from other liberal conceptions; and, it is expressed in an abstract and general way. ${ }^{2}$ In the absence of a clear and realistic way of achieving a universally justifiable conception of liberalism, Dworkin settles for identifying in his conception those qualities that he would expect to find in the legitimate liberal core. This approach is more modest than those of Rawls and Nozick; it seems reasonable, although Dworkin would open himself up to criticisms of these virtues, and to accounts of different approaches that seem to satisfy them equally well.

\footnotetext{
${ }^{2}$ See above, chapter 4 , note 12 .
} 
Dworkin's more significant contribution to liberal theory is in his attempts to characterise the social and institutional meaning of equal concern and respect. His inquiries lead him to reject welfare measures of income and, instead, to embrace resource equality. This equality is given substance from his hypothetical narrative of shipwreck victims, using the auction and insurance devices. The auction, and the requirement that the outcome satisfies the envy test, so that no person envies the bundle of any other person, is manifestly equal. The subsequent operation of the insurance market, however, never quite functions in the way that Dworkin intends. Instead, it seems that individuals would, conceivably, take out a different level of insurance than he suggests. The operation of the insurance market seems not to reflect adequately an outcome of equality in every situation. Dworkin imbues those acting in the hypothetical insurance market with impulses that they need not have necessarily. Furthermore, he fails to account for the full complexity of distributional circumstances, and what this demands of a complete conception of equality, as well as the complexity of moral principles that are potentially relevant to these diverse circumstances.

Dworkin's attempts spawned a substantial literature that aims at searching for a morally universal and complete expression of distributive equality. These embody approaches to and conceptions of equality that are varied and sophisticated. The brief discussion of these suggests, however, that none has managed to overcome the complexities of distributional moral concerns; and that, like Dworkin, none has managed to justify their own equality conception as being the true liberal core, the legitimate central concern of liberal theory.

Like Dworkin, Ackerman is interested in the role of neutrality and equality in liberal theory, but his focus rests much more firmly on the former than the latter. He also employs a hypothetical narrative, focusing on a group of people reaching a new world; and, he is primarily concerned with the distributive outcome of the resulting dialogue. The key principle that directs that dialogue is of neutrality: that no-one can justify their claim to more than equality by reference to the superiority of their conception of the good, or of themselves. This limitation on dialogue, he argues, leads to the result that no person can validly claim more than anyone else. The neutrality principle is, in effect, Ackerman's attempt to devise a rule that is morally valuable and 
open, in the sense that it is not biased in favour of any person's personal interests, and that leads to a definite distributive outcome.

Ackerman, however, cannot achieve the neutral result for which he aims. The idea is that a distribution of resources is moral if it is insensitive to personal beliefs and goals, and, so, does not advantage or disadvantage any individual. This approach fails because Ackerman's principle is not neutral in the way that he desires; rather, it works from the baseline of a material equality, which may, in many ways, be fair, but is not neutral in the way he desires. Neutrality offers no reason to prefer, in Dworkin's terms, this form of resource equality to a utility-based measure of welfare equality. It seems that any expression of equality implies particular moral values, and that no particular, concrete expression can avoid the problem of contestability. Even if Ackerman's expression of equality succeeds, it is questionable whether the focus of justice should be on justifying as neutral a particular material distribution. Such an approach excludes other potentially relevant liberal concerns, such as the Rawlsian focus on matters of liberty and the right.

Ackerman's final contribution to recent liberal theory concerns his approach to justification. Liberalism, he argues, does not stand on one universal foundation, but, rather, is reached by various paths in a philosophical debate. Each of these is strong enough to support an adherence to liberal ideas, but they are also complementary. They respond to issues surrounding the corrosiveness of power, the doubt as to the right and good, the need to respect individuals' autonomous conceptions of the good, and the scepticism provoked by the attachment of moral meaning to our beliefs and desires. In many ways, Ackerman seems to bridge the early and the postcommunitarian liberal theory, and this is most obvious in relation to his promotion of dialogue, both as the subject and as the justification of liberal theory, and in his presentation of an argument for liberalism that is more open to difference. Unfortunately, this dialogical and multi-stranded approach to justifying liberalism is not supported by an adequate meta-ethical explanation of these plural paths to liberalism and the role of dialogue in reaching a true conception of justice.

In general, these early contemporary liberal conceptions aim at universal justifications, but fail in that they build structures that contain, implicitly, the values 
and ideals that they are attempting to justify. Each employ particular priorities or focuses that are mistakenly perceived to be of fundamental significance to justice. Rawls' priority of the right over the good, Nozick's natural rights, Dworkin's equal concern and respect, and Ackerman's neutrality principle are all engaging and plausible moral doctrines, but each presupposes what the respective thinkers should be justifying, and ignores the contestability and the complexity that characterises the domains in which they operate.

\subsection{Post-communitarian liberal theory}

Communitarians criticise the bias in liberal theory, expressed in implicit conceptions of the self or the good, and in the general enlightenment approach to moral theory. These criticisms were important to the development of liberalism, and engendered a number of shifts in approach. Perhaps the two most significant of these were, firstly, the move to treat difference within a society as being central to the justification of liberal theory and, secondly, the move to contextualise these justifications so that they are relevant to contemporary or western societies. Both of these can be seen as not just acknowledging moral contestability and complexity, but as finding, in some form of this uncertainty, the source of the power of liberalism. In other words, such theories seek to show that, for our western societies at least, the diversity of rationally irreducible thoughts and beliefs leads one to favour liberal values and institutions.

The clearest expression of this effective reversal in liberal justification is to be found in those conceptions that place pluralism at the centre of the liberal matrix. The theories of Walzer and Raz represent the first moves towards this position. Walzer's principle of the separation between spheres of justice turns out not to have the power that he ascribes to it, but his focus on the importance of the social understanding of goods, and the contextual nature of his justification, are both engaging moves in liberal theory. His use of social meaning draws the criticism that he is merely interested in what our beliefs and values are, rather than what the content of a conception of justice should be. This argument is not entirely effective, in the sense that Walzer is searching for an expression of justice that acknowledges and is 
informed by the social context and meaning of those goods that are its subject.

Despite this, he offers no adequate account of how justice is to be drawn out of this meaning, and his own attempts to do so seem to reflect his personal moral beliefs.

Raz adopts a contextual account that identifies autonomy as the value of western society that is central to justice. He argues that the implementation of liberalism necessarily involves perfectionism, the promotion of a particular conception, or set of conceptions, of the good. This, however, does not mean that only some ways of life are permitted in a liberal society, but, rather, that certain repugnant ideals are excluded. This approach leaves open a space within which minimally liberal citizens can express a plurality of incommensurable forms of life. This acceptance of pluralism, of the inability to rationally determine between different conceptions of the good, sets the landscape within which individuals can exercise their autonomy. There are two principal problems with Raz's approach: firstly, that this autonomy does not cohere logically with his perfectionist approach; and, secondly, that the central value of autonomy is justified only in a contextual way. Raz needs to make the value of autonomy, and the restrictive effect of his perfectionism on autonomy, cohere; if the former is central to justice, then the seeming priority of perfectionism needs to be explained.

Contextual justifications represent an important post-communitarian approach to liberal justification. Theories, like that of Raz, that draw on a particular element of contemporary western society, need to offer a historical and sociological account of this element. This must be sufficient to ground its importance in the resulting theory. This sort of justificatory strategy is disconcerting in that it chooses a particular feature from the normative complexity of a diverse society. Raz's approach of championing the western value of autonomy is subject to these criticisms, and, consequently, its place as the central liberal value seems contestable. A better approach to contextual justification is to identify, as the relevant feature of western society, a diversity and plurality of values. This is a feature of Gray's post-liberalism, and has the advantage of reflecting, fairly, the normative complexity of western society.

Gray's justification of western liberalism rests on its promotion of diversity, and western freedoms and autonomy. Unfortunately, he offers no argument as to why the 
fact of pluralism should mean the moral value of increasing diversity. Although diversity might indicate that legitimate pluralism has been allowed to develop, this does not mean that liberal states should actively promote diversity. In addition, the contention that western liberalism promotes western liberal freedoms holds little moral value, and seems only to express the idea that commonly held conceptions of justice should be relevant to the system of justice. As such, it suffers from many of the same contextuality issues as Raz's autonomy. However, these issues are not as pronounced, since these values are secondary to pluralism in Gray's system.

Gray's own thinking developed from the post-liberal position, on the grounds that such an approach does not fully express social pluralism, and, in fact, entails some liberal limitations on expressions of human flourishing. This idea mirrors the pluralism of Kekes, who argues that the overridingness of particular liberal values contradicts the full acceptance of the pluralism of values. His analysis is limited, in the sense that his focus is on the earlier liberal theories, which all tend to have substantive, singular focuses. He ignores, therefore, liberalism that is founded on social difference. He also fails to acknowledge the close affinity between liberalism and pluralism; that the freedoms that are central to liberalism allow and engender real value pluralism.

This affinity forms the central argument of liberal pluralism. Galston conceives of a thoroughgoing value pluralism that defines the nature and shape of moral value. $\mathrm{He}$ then raises the presumption that, since coercion is contrary to the rational incommensurability of pluralism, the state should not interfere with an individual pursuing their own conception of human flourishing. This presumption, however, is not a general moral rule that can be drawn wholly from the recognition of pervasive value pluralism. Instead, it depends on assumptions about how this conception of pluralism is applied between individuals, and, also, on the presumption that this occurs in a conflict-free environment. Both of these points mean that something more than value pluralism is required in order to justify a general presumption against interference. This is a general problem of liberal pluralism, that the fact of pluralism, itself, does not necessarily imply a particular moral response. Instead, some other moral value, such as respect or autonomy, is required in order to get from pluralism to liberal principles. This result is not restricted to liberalism, but applies, also, to the 
purportedly purer forms of ethical pluralism suggested by Kekes and the later Gray. The requirement of additional moral value presents a particular problem for Galston. This is because any additional moral content conflicts with the assertion of a fundamental incommensurability between moral positions. In other words, his presumption against a state imposing particular value judgments, seems to actually require such judgments as part of its justification.

Pluralistic approaches to liberalism suffer from a major difficulty, namely, that of getting from a fundamental expression of irreducible difference, to a singular, definite conception of liberalism, in a way that is relevant across contemporary western society. In this way it, such attempts must face both the moral contestability implied by a particular account of pluralism, and the social and moral complexity that characterises a pluralistic society: the former suggests that, if values are plural and incommensurable, then liberal values should not be preferred; and the latter emphasises that difference is not easily reduced to unity. The various criticisms that stand against the above theories are, either, particular expressions of this general problem, or, are closely related to it.

Political liberalism also works from the fact of pluralism, but operates in a different way. Its basis is the separation between the political and the personal, where liberal values are prominent in the domain of the former, and in the use of public reason to overcome disagreement in the political sphere. The point of Rawls' political liberalism is to justify a liberal approach to those western people who are reasonable, in that they adhere to a democratic political culture, and are willing to co-operate with others in the political sphere. This means the setting aside, or, at least, the downplaying, of their personal comprehensive beliefs in order to achieve an effective political agreement. The power of this approach is expressed in the nature of its contextual justification. This is not limited to western societies, but, rather, to the reasonable members of these societies; in other words, to those who are already predisposed to western democracy and who desire political cooperation and effectiveness. This, however, should not be read to include only those who are already liberal; instead it includes those who hold various, divergent personal beliefs, providing that they also hold reasonable political beliefs, and are willing to accept that their personal beliefs will not direct their society's political process. The power of 
political liberalism, then, is the justification of liberalism to reasonable, democratic citizens.

Rawls' expression of this approach, however, does suffer from a number of difficulties. His rejection of philosophy and metaphysics is relevant in that he is presenting a theory that is based on a political pragmatism on the part of those who are willing to subscribe to it. This cannot, however, deny the importance of the theoretical nature of Rawls' description of political liberalism, nor the relevance of the truth of its various assertions to its justification. This general point aside, Rawls' system is based on the separation of the political and the comprehensive, and his conception of reasonableness. The former plays much the same role as does the priority of the right over the good in his earlier work: it separates the space within which we are free to adopt and act upon our own beliefs, from that which comprises and results in liberalism. The latter excludes from consideration those who do not meet its requirements. This is problematic for Rawls in that his project depends on the exclusion of those who do not agree with it, and a justification of this, as well as an explanation of how it would work in a diverse society. In addition, Rawls' own conception is too strict in that it includes recognition of the 'burdens of judgment', which would effectively exclude many of those who should be an active part of the politically liberal state.

Political liberalism attempts to overcome the bias implicit in earlier liberal theories by acknowledging it, and limiting its justification accordingly. There are doubts, however, as to whether an overlapping political consensus would actually result, and what value this would have, given the exclusion of the unreasonable. As with the distinction between the right and the good, Rawls assumes that there is enough in the meaning of the political to build an effective consensus, and that the conception of the reasonable is broad enough to make that consensus meaningful. Rawls' approach seems to fail, in its characterisation of the political, and its definition of reasonableness, but the approach of political liberalism seems an engaging attempt at a contextual and pragmatic liberal justification.

Larmore's expression of political liberalism is itself interesting, particularly since he embraces moral theory, and actually founds his system on three sources of moral 
complexity that are often ignored or downplayed. In relation to the substance of his theory, he more clearly identifies the values that underlie his justification of political liberalism than does Rawls. These include, in particular, rational dialogue and equal respect. He argues in favour of a conception of neutral political neutrality, but this depends heavily on the distinction between the right and the good, and on the retreating to neutral ground which, even if effective, could lead to disturbing results. Larmore's focus on the values that draw political liberalism from reasonable pluralism helps to explicate the source of the substantive principles of the former. In this way, he opens political liberalism up to the claim of plural and contestable value, instead of relying on the claims of a common democratic culture.

It is this contestability of the public domain that informs Gaus' criticism of political liberalism, and his preference for placing public reason at liberalism's core. It is in the face of persistent moral agreement, rather than as to conceptions of the good, that Gutmann and Thompson recommend an approach of public reason through deliberative democracy. They set out a process of political dialogue that engenders the working-through of moral disagreement. While more a theory of liberal democracy than of liberalism, their approach does raise a number of important issues with liberal conceptions of public reason: the tension between substantive liberal values, and the open content of the political dialogue; the difficult relationship between dialogue and political theory; and, the role and effect of the dialogical process.

Gaus' theory, in contrast, has a clearly liberal flavour. His central contention is that coercive state action is legitimate when it is justifiable to the person who is to be coerced. This does not mean that actual justification is required, but, rather, represents a complex epistemological standard by which a belief is superior to an existing belief for that person. This approach is supposed to reflect a liberal core, while allowing that liberalism can take on various political expressions. The problem with this process is that coercive moves lead to inconclusive justificatory outcomes. In many of these cases, a democratic umpire can be used to determine the outcome, but this approach seems to undermine the very concept of public justification. The underlying difficulty is that, in practical terms, political agreement is not a realistic outcome of public political reason. Particular limitations can be applied to minimise the disagreement, such as are used by Rawls and Gutmann and Thompson. 
These, however, will never be able to remove disagreement completely, and are more in the nature of devices that focus the dialogue on particular issues and remove morally irrelevant concerns. This leaves the public reason theorist with an irreducible disagreement in the domain of the political, and it is at this point that democratic tools are often employed to break the deadlock. While public reason performs a very powerful role in shifting complexity and contestability from the theoretical to the political realm, public reason theories must be able to deal with this disagreement in a way that is itself moral, and is consistent with the remainder of the theory. This is one of the justificatory challenges of a public reason conception of liberalism, along with the requirements of showing that the particular approach to justification is morally legitimate, and that public justification itself is central to liberal justice. Gaus' approach represents an enterprising attempt at satisfying these, but, ultimately, he fails to argue convincingly that his political process will reach outcomes that are morally valuable.

Multicultural demands for recognition represent a significant challenge for liberal theory. They imply that its individualistic focus means that difference-blind liberalism is inadequate to deal with cultural minorities in a just way. The typical liberal defence to this claim is that the ability to deal with pluralism is an essential aspect of liberalism. Within a Rawlsian framework, an individual's cultural adherence is expressed through their freedom to associate and to determine their own conception of the good. The multicultural reply is that this approach does not treat minority groups fairly, in that the decisions and assumptions that states make tend to favour the majority culture over the minority, especially given the nature of the relationship between individuals and their cultures. This argument is valid in that liberalism is expressed in particular political and institutional forms that tend to assume default membership in the majority culture. This point is deepened by the idea that liberal bias and contextual liberal justifications could both favour majority over minority cultures.

The need of liberalism to incorporate multicultural values raises the difficulty of recognising rights that do not apply equally to every individual. Approaches to this can, either, append group rights to existing liberal structures, or, change the very 
conception of liberalism to one that is more sensitive to cultural difference.

Kymlicka's Multicultural Citizenship typifies the former approach. In it, he makes autonomy- and equality-based arguments for granting rights of fair accommodation to immigrant groups, and rights of self-government to national minorities. In relation to national minorities, he observes that one's societal culture gives meaning to the exercise of autonomy, so that the adequate exercise of autonomy requires that the state maintain and promote such cultures. In addition, because of the cultural bias inherent in particular government decisions, equal treatment actually requires that minority cultures are treated differently than majorities. This approach raises two difficulties: firstly, that Kymlicka's conception of societal culture is monolithic, and ignores the potentially plural nature of our cultural adherence, as well as the interactions and interdependence between cultures; and, secondly, that he restricts the operation of liberal cultural bias to a small number of government decisions, when it might apply to the operation of liberalism generally. Both of these raise doubts about the cogency of Kymlicka's multicultural liberal structure.

The theories of Tully and Parekh reach beyond normal liberal conceptions, and attempt to build a truly multicultural conception of justice. Both argue in favour of an intercultural dialogue that is political, and for a parallel dialogue between liberalism and other political values and traditions. For Tully, the history of constitutionalism shows a bias against diversity, especially cultural diversity. His response to this incorporates a complex conception of culture that emphasises internal diversity, and the interdependence of cultures. It is based on a respect for the success of a culture or tradition, in terms of the continued adherence of its members, and results in a system that promotes diversity and the acceptance of difference. The obvious problems with this result relate to: Tully's definition of culture, which gives so central a place to cultural interaction that it loses sight of the strong relationship between individual and culture; and to, the difficult criticism concerning his failure to conceive of culture itself as being a culturally contingent notion.

Parekh, in contrast, emphasises that there exists a shared humanity that is mediated by culture. As with Tully, he presents a complex conception of culture, which includes variations in the nature of cultural attachment. He also applies the value of diversity to justify the intercultural dialogue, and emphasises the importance of being reminded 
that the approaches of others are as valid as one's own. Parekh claims to move beyond liberalism, but the rules and principles that inform his dialogical approach seem remarkably liberal. In all, both of these theorists can be seen as endorsing a multicultural liberalism that does not simply hold liberal values as primary, but, rather, incorporates both liberal and multicultural concerns into their systems. The most significant contribution of multiculturalism to liberal theory is the realisation that there is a cultural dimension to liberalism: that particular liberal expressions of justice are founded on and informed by their cultural context; and, that differenceblind liberalism is not adequate to ensure that liberalism treats equally the members of minority and majority cultures. Multicultural demands identify the cultural component of issues of moral contestability, and challenge theories that are implicitly unifying, or that depend on agreement within a society.

The final form of liberalism relates to postmodernism. There are few postmodern attempts at liberalism, perhaps because of the reticence of postmodernists to engage in the promotion of substantive moral theories. This field is dominated by the work of Rorty, and his theory of ironic liberalism. Rorty declares the unavoidable contingency of self, of society and of truth. The latter, he argues, is not found and recognised, but is constructed out of the vocabulary of a particular linguistic community. Rorty's liberalism is not derived directly from this contingency, but, rather, is nonfoundational. It is based on western moral and political values, and the pragmatic idea that people will adopt a description of liberalism that will allow them to interact with their environment in the most effective way. From these concerns, he devises a liberalism that represents equality between ironic self-creation in the private sphere, and the avoidance of cruelty and humiliation in the public, political sphere. As an explicitly sceptical liberalism, however, Rorty's conception faces two difficulties: that the statement of contingency will be self-refuting, in the sense that it is itself noncontingent; and that getting from contingency to a particular expression of liberalism will prove difficult. To the first difficulty, Rorty has no adequate response. His approach to the second is to eschew a direct link between contingency and liberalism; but, this makes it difficult for him to assert his conception of liberalism as being superior to any other. He relies on pragmatism and on the seeming universality of solidarity and ironism, but these seem to be both contrary to contingency, and too broad to be able to distinguish from among alternatives conceptions of liberalism. 


\section{Responding to moral uncertainty}

These theories of liberal justice all seem to represent failures, in one form or another, as responses to moral uncertainty. In particular, their justifications seem inadequate to establish the distinctive value of a conception over its alternatives, and their conceptions do not seem to fully reflect the complexities of our moral and social lives. This is not to say that all are without value: they serve to deepen our understanding of liberal morality and of liberal political practice; they offer engaging conceptions and arguments as to what liberalism means and how it might be manifested; and, they present particular values and approaches that, by virtue of their expression, we might choose to adopt as our own beliefs. The point is that none of these liberal theories amounts to a conception of liberalism that we could recognise as being its best expression, and that none is successful in meeting its own aims. The task for this thesis is to look at some general ways in which these theories collectively respond to moral uncertainty, and, then, at possible future directions for liberal theory.

\subsection{Liberal duality}

Liberal theory functions by defining a liberal space, within which individuals are able to choose what to believe and how to live. Of course, any political theory necessarily defines freedom for its citizens, whether explicitly or implicitly, but the difference is that for liberalism this space is its central focus; it is central to liberalism that people enjoy a substantial freedom. No theory of liberalism, however, can allow people a total freedom of choice. Such an approach would, either, reflect a free-for-all similar to a state of nature, or, could contravene the limitations and conflicts inherent in any situation over which a conception of justice is to be imposed. Particular conceptions of liberalism are differentiated, not by the fact of liberal space, but, by the nature of that space. This nature is defined by the characteristics of the theory itself: its ideals, values and principles. These convey the nature of the freedom afforded to people, and its limitations. This means that, as has been suggested at several points in this thesis, liberalism is, in an important sense, both open and closed: open, with respect to the 
freedom that it champions, and closed, in that this freedom implies particular ideals and, so, limitations on freedom.

This suggests a fundamental conflict inherent in liberal theory, between its openness and closedness: between the idea of a legitimate diversity of beliefs, preferences and actions, and the agreement of beliefs required to justify the characteristics of a particular liberal conception. Rawls' original approach to resolving this was to apply the fundamental moral distinction between the right and the good to reflect these different, open and closed, parts of liberalism. The system that this expresses reflects the essential beauty of liberalism: that we could all recognise our own conceptions of the good as being contestable, and, so, could agree to allow each of us to choose and live our own conceptions. The latter point informs the essence of the liberal meaning of the right: the rational agreement to allow diversity and freedom in relation to the good, which is itself moral and universal. Such a system, however, does not survive close scrutiny. The characterisation of the good as necessarily being morally inferior is not easily sustainable; and, the principle of the right is too broad to specify particular liberal principles, and so Rawls adds various moral ideas of equality and liberty. In doing so, Rawls ignores the moral contestability of many of these ideas, and assumes, mistakenly, that there would be agreement in the original position, and as to his proposed reflective equilibrium. More generally, his approach does not seem to reflect the complexity of moral issues of the right and the good.

This pattern pervades much of contemporary liberal theory. The idea of 'conceptions of the good' itself is common, and indicates those sets of beliefs we hold that are accepted as being individual, contingent and contestable, in the manner of personal preferences rather than the recognition of universal truth. It is used to carve out an area of legitimate diversity, and, by definition, to separate it from the universality on which the theory itself is founded and justified. In terms of other early contemporary liberal theories, equality means that each person is given an equal opportunity to choose and pursue their own conception of the good, and neutrality means that the coercive action taken by states will not be sensitive to particular conceptions of the good. Both of these imply a respect for the choice of conception, but both can be criticised for requiring additional substantive and contestable moral content, and for 
readily distinguishing these matters of the good from the moral matters that actually inform the particular conception of liberal justice.

This analysis could also be applied to difference-based forms of liberalism. The shift in approach that is implied by this group of theories means that the difference itself tends to be conceived more generally than with the earlier approaches, and it takes on a new justificatory role, but the basic analysis is still relevant. For value pluralism, for example, the conception of pluralism defines the diversity, and the incommensurability between values makes this diversity legitimate. From the perspective of the duality between the openness and closedness of liberalism, this approach faces two problems: that the liberal structure is not necessarily implied by pluralism, and, instead, is merely one among many possible responses; and, that the values that underlie the particular response that the approach prescribes cannot easily be distinguished from those values that are the subject of the pluralism.

Resolution of this tension demands that a liberal conception be justified on terms that are separate from the realm of legitimate diversity. Because liberalism of this form is moral in nature, justification requires the promotion of particular moral values. Even if these values are separated from the diversity, the fact of pervasive moral contestability means that there can be no single value or set of values that have sufficient content so as to necessarily define a particular conception of justice. This in itself suggests that moral beliefs should not be outside of the domain of legitimate diversity. In this way, the tension between the openness and closeness of liberal theory seems insoluble.

\subsection{Intuitions in liberal theory}

The role of our moral intuitions in liberal theory constitutes another general difficulty in relation to uncertainty. The nature of the problem concerns the moral meaning and value of incorporating these intuitions, and, in particular, of the potential conflict between our moral judgments and values, and the values that are proper to justice. Since Rawls' A Theory of Justice, actual moral intuitions have played a key role in the 
structures and justifications of liberal theories. This is explicit in the theories of Rawls and Dworkin, for whom authenticity, or reflecting commonly held intuitions, is key for the justification of their liberal conceptions. Later theories involve our moral intuitions in various ways. For Walzer, for example, intuitions form part of the social meaning that underlies goods, the subject of his justice. Raz's approach is founded on the contextual social value of autonomy, which is itself a moral judgment. For some later theories, differences in intuitions are part of the diversity of western societies. This is most obviously true of value pluralism, but also applies to theories of deliberative democracy and justificatory liberalism. It is also true of Rorty, although the focus of his work is more on the contingent nature of morals, rather than on difference or diversity.

For liberal theories that incorporate moral intuitions, it is important that the role of these is explained in a way that is compatible with our understanding of morality, and that coheres with their account of liberalism. One of the most engaging approaches is that of Rawls' reflective equilibrium. What is interesting here is the interplay between our considered moral judgments and the conditions and outcome of the original position. Essentially, Rawls is directing his readers to consider, morally, his formulation of the original position, and he anticipates that they will reach a position of agreement with him. He does not just rely on existing belief about liberalism, but, rather, encourages close consideration of his liberal conception. Although he sets out a plausible and attractive system, he does not attain the intuitive agreement that he seeks.

The approaches of those later theories that allow for a plurality of legitimate moral judgments, all tend to emphasise the importance of an actual political dialogue as part of their substantive liberal content. This makes sense, in that it is only dialogue that can account for a diversity of moral intuitions. This is true of both public reason theories, and of the multicultural approaches of Tully and Parekh. These theories have the virtue of being able to incorporate diverse moral judgments and, by implication, moral contestability, in an effective way. They then face the problems of justifying the process of political dialogue itself, since this depends on contestable moral values, and arriving at some meaningful result. In relation to the latter, Rawls' political liberalism is the only approach that imagines that a consensus will result, and, even 
with substantial restrictions he imposes on reasonableness, there is doubt as to whether this could actually be achieved.

Overall, the role of intuitions in liberal theory is difficult. There is a sense in which they must form part of any reasonable attempt at liberal justification, since otherwise there seems no moral fixed-point or framework from which to view different approaches. The only alternative to this approach would be to attempt a justification based on values that are simply declared, such as with the natural rights of Anarchy, State, and Utopia. It is clear, however, that there is a substantial diversity of intuitions, and that any restrictions placed on these, so as to achieve moral agreement, would involve specific and contestable moral values. The theories that accept this diversity prescribe an open moral-political dialogue, which is useful in that it seems to relieve liberalism of a great deal of moral contestability and complexity. This approach, however, still faces the considerable problems relating to justification and the role of dialogue. A Habermasian approach to discourse ethics, whereby dialogue actually assists groups in reaching morally universal results, would mean that these two problems, in effect, cancel each other out. This is an attractive result, but depends on the meta-ethical assertion that genuine dialogue achieves morally universal results, which is contrary to the experience of persistent moral disagreement. None of the liberal theories canvassed make this argument, although some do suggest that dialogue has the positive effect, not just of expressing diverse beliefs, but of engendering a mutual understanding that is positive for justice, even if it does not amount to actual agreement.

\subsection{Biased conceptions of liberalism}

The final point to be made about the general nature of the liberal response to moral uncertainty concerns the bias that pervades liberal conceptions of justice. The early contemporary theories seem to offer plausible, but, ultimately, biased, expressions of liberalism. Later theories suggest a more contextual bias, and multiculturalism, in particular, accuses liberalism of a bias related to western, cultural values. The nature of this bias is that a liberal conception, or its justification, contains moral content that 
is not morally relevant, or does not reflect the moral power and value that is attributed to it. The above discussions on moral diversity and contestability suggest a general inability for liberal theory to reach a particular prescriptive result in a morally valid way. This is pronounced in relation to liberal theories that aspire to universality, but also applies to contextual justifications, since these are subject to criticisms from diversity. Implicit in this claim, and in this thesis generally, is the idea that a liberal theory needs to show universality or demonstrable power over alternative approaches, rather than just demonstrate some positive moral worth. This is consistent with the approaches and attitudes of the theories considered, even in relation to the justification of liberal redescription in the context of contingency.

This seems to apply, then, to liberal theories generally, and reflects both of the general comments on liberalism as a response to uncertainty, above. Together, they suggest that liberal justification depends on moral assertions that are themselves part of a contestable and highly complex space. This applies, also, to conceptions of particular liberal ideals. Liberal expressions of equality or neutrality, for example, are themselves moral accounts and, so, are potentially complex. This suggests that no particular conception of equality or neutrality could be justified as being the best liberal conception, since they would rest on particular moral values, and need to be set out in a detailed way in order to be effective.

These three points offer a picture of the nature of liberalism's response to moral uncertainty. Together, they suggest that liberal theory is unable to justify itself in a general and compelling way, and, instead, is susceptible to the recognition of the contestability, complexity and contingency of our moral judgments, and our moral lives. This is not to deny that a morally correct position can exist, or that there is a conception of liberalism that represents the most morally valuable given the circumstances in which we find ourselves. Rather, it means that moral theory is not capable, in conjunction with our moral beliefs and intuitions, of reaching a recognisably moral, singular and complete conception of liberal justice. More than this, it suggests that the disagreement over such conceptions is not entirely the result of mistake or misjudgement, but, rather, reflects the inability to rationally determine between moral values, and to rationally reduce complex moral concerns to a simplified structure, and with a set of simple principles or rights. 


\section{Developing liberal theory}

\subsection{The problem with liberal theory}

The central observation of this analysis, then, is of the persistent failure of contemporary liberal theory to respond adequately to arguments from moral uncertainty. This is despite a keen awareness of the nature of the arguments involved, and concerted attempts to overcome or, at least, to ameliorate their effects. The challenge of moral uncertainty concerns more than disagreement about the central liberal value, or the best characterisation of a liberal conception of justice, but rather affects its very structure and mode of justification. This would seem to suggest that contemporary liberal theory will inevitably struggle to make any claims that are morally valuable. But this conclusion is belied by enthusiasm and interest that the field generates, which is academic, but also moral. In relation to the latter, much of the substantive principles of the various liberal conceptions remain readily recognisable as positive expressions of morality and justice, and retain the power to challenge and inform sincerely held beliefs. This is the case despite the doubt shown about the theories' central structural principles and justifications. This seemingly paradoxical relationship, between the failure of liberal theory from the perspective of moral uncertainty and its intact moral value, may seem initially to raise doubt about the value of either the perspective of moral uncertainty, or of the practice of liberal theory. But ultimately, I suggest, it points the way towards potential improvements in the latter; towards a conception of liberalism that both responds cogently to moral uncertainty, and acknowledges and promotes the moral power and value of liberal theory.

\subsection{Liberal theory and value}


The analysis set out in this thesis understands a conception of liberal theory to mean a detailed characterisation of justice and social organization that holds at its centre the idea of a necessary liberal space; a space within which people are free to act as they choose, and are free from state interference. The justification of any such conception requires some general reasoning and explanation for the nature and extent of that space. The characterisation of the central aspects of liberal conceptions as being freedom and justification has been recast as their openness and closedness, respectively. This approach emphasises one of the central themes of an uncertaintybased appreciation of liberal theory: that liberal freedom is not at large, rather it is confined by principles and ideas that must be clearly defined, and that necessarily restrict other freedoms. The general and persistent problem of contemporary liberal theory is that there seems to be no universal value or values that can clearly define this liberal space; that any likely candidates are either morally contestable, or are expressed so broadly so as to be unable to justify definitely any one system. Even attempts at holding the pluralism or contingency of values as this central idea seem to rest on contestable grounds, and never achieve the universality of result that they assert.

This analysis seems to leave liberal theory without an obvious foundation or source of value, which in turn leads to a questioning of the nature and merits of the Western predilection for liberalism. Without a clear theoretical answer, the search for the value of liberal theory naturally turns to the social and historical. Liberalism captures a general understanding of a Western set of political and moral beliefs and practices. Without an adequate theoretical foundation, liberal value can be founded on and expressed through this common Western understanding of and adherence to its conceptions and principles; and through the captivation of people by its expression as a political conception and a political philosophy.

It is this real-world appreciation of liberalism that can be used to envisage a valid liberal response to moral uncertainty. Much of the analysis in this thesis, however, shows how it cannot be used as a source for liberal theory, in the sense that the latter is the fully-formed and natural expression of the former: there is no attempt here to derive some conception of liberalism directly and necessarily from Western belief. Such an approach would require the identification of a central value or set of values, 
similar to the contextualised liberalisms of Raz or Gray. Nothing in our sociohistorical beliefs, however, is so strong and so singularly definite to qualify as such a value, to the exclusion of all others. What can be allowed, is that liberalism is central, and perhaps paradigmatic, to Western political belief and thought, and that its values are equally important in Western political practice, at least in relation to foundational matters. The relationship between the matrices of Western moral and political belief, and a particular expression of liberal theory, then, is not as between the pressing down of a plunger and the resulting explosion: it cannot be that obvious or that necessary. Instead, our belief is more like the terrain onto which a conception of liberalism can be mapped and built. Not just in the sense of being a flat piece of land, but as a large, complex and varied landscape, whose features and details influence the structure that can be build upon it. But while the structure must begin with and come from the terrain, it can take on forms that build onto it in various ways, and express various themes and modes of design. The terrain is still foundational, in the sense of being fundamental to the structure, but there is no sense of necessity in the relationship between it and a particular structure built upon it: the terrain might be said to have the potentiality for the structure, but the latter is not inherent in it.

The point here is to develop the nature of liberal theory and justification, by recharacterising the relationship between theory and its foundation. Because that relationship is not a necessary one, this approach will not single out any particular liberal conception. Rather, it sets out a framework for liberal conceptions that build upon Western liberal, political and moral beliefs. This being the case, there is no claim that any resulting conception is correct, or represents the whole of justice; in the context of belief, it is not possible for one conception of liberalism, or even liberalism itself, to achieve this result; the design of a structure does not represent or capture the whole of its terrain, but rather it builds on it, borrowing from and using those features that suit it. Instead of incorporating our considered moral intuitions into a settled structure, this approach acknowledges their broader role in driving the content of liberal conceptions, but without assuming those intuitions are so well-defined and uniform as to necessarily indicate the correctness of one approach, or of the focussing on one value. This is a more cogent expression of the relationship between intuitions and theory, and describes why theory is never final, perfect or whole, and yet is 
contingent and dynamic: because it is founded on a complex set of beliefs that are themselves contingent and dynamic.

This is not to say that liberal belief is so open and complex as to be without any real content. In fact, it is described by a number of deep and rich values, which could be organised loosely into three categories: the avoidance of wrong, which usually means interference by the state, or of harm caused by the state or by other people; respect, which emphasises the value of each individual, and may mean a focus on equality, neutrality, or the power of consent; and the promotion of autonomy, which takes a more positive attitude to freedom, insisting on the space for the individual to create, or on self-determination. These values are overlapping, and can be mutually enforcing or potentially opposing. Often one of these will be used to justify another, and in the same way, much of contemporary liberal theory attempts to reduce these values to one central liberal concern. But all such attempts fail to recognise the contestability of the value involved, and the complexity of the relationship between the different values. So, although each such conception does convey something about Western understandings of liberalism, these values cannot in fact be reduced to a single concern or principle, if that is supposed to represent the whole of liberalism. Yet, particular liberal conceptions do utilise and are informed by the content of these liberal values.

By denying universal justification, and by declaring belief to be the foundation of liberal conceptions, this approach might appear to reduce, or remove entirely, the power of theory. It is at least true that it changes the nature and role of liberal theory, which can no longer find or declare a singular truth about the meaning of liberalism, or its best expression. But, this is not to say that it is left without any comparative power. Liberal theory is still able to identify value, albeit within a plural and complex context; to challenge belief, and to structure intuitions and understandings in meaningful ways. This is not with final authority, and not as a process that overrides or transcends belief. In this framework, there is a complementary relationship between belief and theory (to this, the metaphor of terrain and structure cannot extend). Here, the power of theory is its ability to systematise, structure and arrange that belief, through reasoning and the tools of philosophy. Although theory retains this power, what changes is the meaning of this process. The role of theory within the 
framework is to assist in the examination and the exploration of belief, and to challenge one's beliefs or the beliefs of others. This challenge comes not simply because of verifiable mistakes of fact or logic, but because of the cogency and coherence of one's beliefs, principles and intuitions, usually when confronted with alternative ideas; in other words, it can cause a person to shift from one set of beliefs to another, even though there is no universal or absolute reason for this. The individual who makes such a change does so because of his or her moral understanding and judgment. The role that theory plays in this process is not to reveal a truth, but rather to inform a judgment; to convince of some flaw or inferior quality; to persuade. In this way theory, in its relationship to belief, takes on the nature of rhetoric. It acts to affect actual belief, in the manner of persuasion; to encourage agreement rather than to assume it. And, their universal pretensions removed, this is an interesting way to view contemporary liberal theories: they propose and describe principles, reason back to various liberal and moral values from which the principles are derived, and reason forward to set out their valuable consequences. It is in this way that these works are recognisably liberal, moral and valuable, despite their questionable foundations.

This does, in some sense, represent a reduction in the power of liberal theory: on this reading, theory no longer has the power to discern or declare the correct or best moral result. Instead, it acts only in relation to existing belief and ideas of liberal value. It does, however, retain the power to challenge and influence belief, and it retains its identity as theory; it is not reduced simply to that which persuades, but is instead that which persuades and affects moral belief in distinctly theoretical ways. Perhaps the central mode of this is to compare and draw patterns that represent belief in a consistent way. In describing rationally conceptions of belief, it tests and challenges particular intuitions, and intuitive responses to imagined circumstances. From this, liberal theory can structure and organise belief into general principles and conceptions. This allows liberal theory the power of being dynamic and imaginative, and locates power in the development of ideas over time, rather than simply finding its value in the imagined resemblance of a conception to the final liberal truth. This development, of course, does not necessarily signify improvement, and certainly does not mean moving towards an end position. Instead it is explained as reactive to shifts 
in liberal beliefs and understandings, and as transformative, with the capacity to influence those beliefs and understandings.

By this idea, liberal theory is limited by being unable to express the whole of liberalism, and by having to work in relation to social beliefs and understandings of liberal conceptions. But this approach also describes the importance of liberal thought, and especially those liberal conceptions that challenge and influence the beliefs of people, and the ideas of future theorists.

\subsection{A framework for liberal theories}

What is set out here is a framework for liberal conceptions, each of which is tasked with taking from liberal beliefs and understanding, as well as the strands of liberal theory, and organising the result into a relatively cogent and coherent liberal conception. It is predicated on a strong relationship between liberal theory and belief, in particular on the rhetorical effect of liberal theory on personal moral and political belief. But this is not to say that within this framework the aim and measure of a liberal conception is its popularity, or that persuasion should be pursued for its own sake. Moral value is not inherent in belief in this way. This is another aspect of the relationship between liberal theory and belief. Just as there is no sense of necessity between the two in relation to liberal foundations, there is, similarly, no direct link in regard to moral value; it is not simply that something believed is necessarily morally better than its alternatives. It is rather that moral belief offers a context, not just for the content of a theory, but also for an understanding of its value. Since we cannot discern the correct or best liberal conception, we can at least be guided by our own considered beliefs, and our experience of differences in the beliefs held by others. To be clear, this does not mean that morality and moral theory is directed by anything as simple as mere preference. The latter suggests only the choice between alternatives, in accordance with desire. It ignores many of the complexities bound up with actual moral belief, and, in focussing simply on a person's self-conceived interests, it divorces from morality much of its content. The conception suggested here, that moral belief offers a context or a landscape for moral value, is a more coherent and a richer 
idea. It retains the distinctively and recognisably moral character of belief, and models the latter in a way that is recognisable and plausible.

As has been explored in this thesis, there is considerable difficulty in incorporating moral intuitions into a liberal conception of justice. This is both because such intuitions are never uniform enough to discern a single liberal ideal, and because of the nagging sense of contingency that attaches to them. The first is of little concern here, since many liberal conceptions are permitted. The second remains, and with it the difficulty of drawing the link between what we do believe morally, and what we should believe, or what it is best to believe. The framework for liberal conceptions incorporates the central role of actual belief, certainly a view encouraged by the difficulty of engaging in theory whose value transcends belief. The separation between belief and moral value is significant: it leaves space for theory to be conceived of as rhetorical, yet still be theoretical, valuable and dynamic; it does mean, however, that that the nature and content of moral value is left disturbingly unspecified. In other words, that moral value cannot be drawn necessarily from belief leads to the conclusion that there is no definite and verifiable means of it being realised, and no viable test to tell when it has been. Difficult though this result is, it should be remembered that the system itself is built on the capacity of the real-world individual to recognise and evaluate moral content within their social and historical context. The moral value of liberalism will not be able to be established any more firmly than this, but, because liberal theory itself cannot, on the present argument, be correct or true, the power that general moral belief offers is sufficient for its intended purposes.

Ultimately, the point of this section is to assert that this is a viable and desirable theoretical framework for liberal conceptions. Its value lies in it ridding liberal theory of false arguments of universality, and so allowing it to work plainly and directly towards more achievable ends. It retains the value of inquiry, of critical thinking, and of theoretical construction, but without much of the theoretical underpinnings and justifications, which are contestable and are contested. Such an approach does not admit the failure of liberal theory, but rather frees it from the need to apply incontrovertible reasoning and find universal principles of justice. Of course, the nature of thinking and theorising is to find similarities between various circumstances, 
and to try and draw general rules from a variety of examples; but there is an important difference between drawing patterns in an admittedly contestable way within a complex context, and claiming for these patterns a universal grounding and a singularity of value.

This framework is itself theoretical, and is already liberal, in that it works explicitly with liberalism. It already assumes ideas of democracy and individualism in the way that belief is incorporated with and reacts to theory. It also incorporates strongly the perspective of moral uncertainty that pervades much of the reasoning of this thesis, and, correspondingly, a sense of openness, and of non-predetermination. There is the risk of this framework itself being perceived as a liberal conception (of liberal conceptions). As such, it would resemble the dual-levelled structure already described: open to various liberal conceptions, but closed as a metaethical theory of liberal and moral value. This is not the intention. It is instead conceived as a framework that arises out of the present social and theoretical situation, and that makes no claims to universality itself. It does, however, claim value: to work with and promote liberalism in a way that is beneficial to its theory and practice. As such, it is inconsistent with but not contrary to alternative conceptions of liberal ethics, nor to non-liberal beliefs.

\subsection{Beyond the framework}

It is possible to look beyond this framework to more substantive liberal ideas. These are to some extent suggested by recent developments in liberal theory, and by the positive content of the framework itself, particularly by its attitude towards uncertainty and consequent openness. Any links between the framework and any such ideas might indicate some affinity, but not a necessity: the framework itself does not dictate all or part of any particular liberal conception. These comments, then, can also be taken to be part of a definite conception of liberal justice, and as such, operate within an explicitly contestable space. 
The first of these relates to the role of political dialogue within this liberal framework. Such dialogue was found to be effective in relocating some of the complexity and contestability of the substance of liberal ideas away from the ambit of liberal theory. The framework of liberal conceptions is designed in response to this complexity and contestability, so it may seem less important that these are relocated elsewhere; because contestability is conceded and assumed at the theoretical level, there is less need for it to be moved to the practical. There is some merit to this observation but, in fact, the incorporation of belief, and its interaction with theory, already resembles a process of dialogue. This general form of dialogue involves a normative discussion on political and moral subjects, and informs and shapes our liberal beliefs. This means that dialogue forms part of the very process by which alternative liberal conceptions are chosen, and more generally, by which liberal values and ideas interact and take political form.

Even though dialogue, broadly understood, functions to inform the theoretical interaction between conceptions of liberalism, this does not render structured political dialogue irrelevant as part of such a conception. The nature of such discussion, and of liberal belief generally, retains a high level of complexity and difference; in other words, the general dialogue that informs belief by no means assumes that there will be a clear winner. For that reason, a more structured and explicitly principled form of dialogue can form an important part of particular liberal conceptions, and would internalise some of that difference into the actual political workings of public discussion. This, of course, cannot amount to an argument that such an approach is the correct or best form of liberalism, but this functioning of dialogue, and its explicit incorporation of uncertainty and difference, would make it an attractive prospect. In addition, the relationship between belief and theory inherent in the framework already anticipates the importance of theory to dialogue: the rhetorical nature of theory makes it an important part of the interaction and exchange between parties engaged in discussion. This relieves some of the difficulties of public reason liberalism related to the separation and distinctiveness of dialogue and theory.

Recognising the inevitability of disagreement in actual liberal discussion raises the issue of the diversity of moral and political belief. It is an obvious difficulty of the framework, since it depends on overall belief, that these are too diverse, too opposed, 
to be reduced to a single conception. The suggested approach has been characterised by moral-liberal belief as being the terrain or landscape upon which substantive liberalisms are mapped. Inherent in this is an idea of belief as being complex and dynamic, diverse and divergent. The issue then is whether this terrain can realistically be said to 'support' any liberal structure, or instead, whether any such structure is so necessarily approximate and singular as to belie its terrain. Perhaps there is some truth to this, but at least this approach offers an understanding of morality that is more accepting of fundamental difference, and the related uncertainty, than any approach where liberalism is based on singular, universal ideas. A second response to this difficulty, less apologetic about the possibilities of liberal theory, would stress that belief is not freewheeling anyway; the necessary interaction and interdependence of belief, and the commonalities and agreements in relation to biological, cultural, linguistic and social contexts mean that a conception of belief simply as an unfettered diversity is not adequate. This is exploited by the framework in considering belief as offering value and power that is more than individual, but without the making either of the mistakes of arguing that a single approach can be distilled from this belief, or that the outcome is universally, or contextually, correct.

This leads to the interesting point, that conceptions based on this framework are naturally majoritarian; they will tend to take most notice of the majority or dominant group or culture, especially with regard to foundational issues. Despite the denial that popularity is the indicator of moral value, it is true that the context of justice will be moulded to the weight of belief. In this way, the framework is, broadly speaking, democratic in nature, and potentially biased, in much the same way as was described by multicultural views of liberalism: matters of justice will tend to be expressed according to their common understandings. It is the nature of liberalism, however, to recognise the arbitrariness of this, and to respond to it in an appropriate way. This inequality or bias, the failure to offer those freedoms, goods and opportunities that are meaningful to their recipients, are liberal concerns, and need to find expression in liberal conceptions. The point is not to attempt to remove this bias, as ultimately liberalism must be manifested through some particular understanding. What is important is that liberalism, by its nature, will be interested to identify the injustice of this bias, and take steps to minimise it. This does not mean that the bias has no effect, any more that it means that the liberalism ceases to be majoritarian; rather, it raises an 
issue to which the liberalism is likely to want to respond. In this way, the relationship between the centre of liberal and social belief, and the periphery, takes on a special importance for current and future liberalism, once the bias of particular liberal forms is admitted.

The two preceding points imply also a conception of the relationship between self and society. With the increased culturally-focussed content of liberal theory, this relationship becomes more prominent. Implied in this is an account of diversity or pluralism in society. These conceptions have proved problematic, as ideas of culture and pluralism are complex. The account of society and self is itself contextual, in that different societies may embody different modes of this relationship. A traditional liberal account of the self tends to focus on its capacity for autonomy, in the sense of creating and defining one's own identity, and for moral appreciation and judgment. Communitarian views of the self emphasise cultural attachment, and, in particular, the constitutive nature of culture. The significance of culture to our identities is also raised by pluralist and multicultural theorists, where the latter tend to envisage a complex account of culture that emphasises the potential to criticise, and to dissent from at least part of one's culture; and differences in cultural attachment, as well as the importance of cultural interaction to both individual and culture.

The relationship between the individual and their society and culture seems to map out a potentially complex structure of interdependence. A better descriptor may be one of mutual embeddedness: self is embedded in society in the sense that its culture and the linguistic community to which it belongs represents a background to its belief and understanding; the society is embedded in the self in that society or culture has no meaning or expression other than that afforded it by its individual members or adherents. The mutuality of this relationship means that neither self nor society is ascendant over the other: our cultures do not constitute entirely our selves to the extent that genuine self-creation is impossible; nor is our power of self-creation so complete that we can readily step beyond our social contexts, and rewrite ourselves from a transcendent viewpoint. In this way, both society and self are fundamental to justice, and neither represents the proper focus or end-point of moral analysis. Multiculturalism adds further complexity to this idea, in that it requires a separation between society and culture, but, this does not negate the central message. Rather, it 
adds the further complexities of cultural plurality and interaction to an account of justice.

This model of the relationship between society and self offers a broad understanding of a complex interdependence. It emphasises on one hand the learned and social nature of moral understanding, and the importance of normative socialisation, and on the other, the personal nature of moral understanding: that it is meaningful for an individual to make judgments independent of the judgments of others, and, so, to hold a particular position that is unpopular or unorthodox, and yet is not necessarily wrong. This model is useful as a part of a liberal conception, and it seems also to add to the framework of conceptions itself; in particular, it informs the understanding of liberal belief implicit in the framework, and affirms the separation of belief and moral value.

One final point that will be made about the framework, and the possible expressions of liberal justice that might be built upon it, concerns the adherence to liberal ideals. Rawls' Political Liberalism envisages persuading those reasonable democratic citizens to adopt public liberal practices, even if these differ from their private beliefs. As such, it offers a model of liberal adherence that is different from the picture of already-liberal Westerners accepting a particular liberal conception as best. Because the liberal framework does not insist on a singularity of approach as do more universal conceptions, it is quite possible to envisage a liberal expression that incorporates a variety of models of adherence to its liberal ideas. In terms of the rhetorical power of theory, this is mirrored by the idea that there can be a number of situations and ways or modes of being convinced by a theory, that are quite various in nature.

This diversity in liberal adherence can be described by two examples, the first of which is the pragmatic liberal, who is similar to that found in Political Liberalism, and other public reason conceptions. Such people, by virtue of their citizenship in a Western society, have a basic moral appreciation of democratic beliefs and institutions, and have the general desire to enjoy the benefits of a co-operative and minimally restrictive political process, will willingly agree with and adhere to a liberal political dialogue. These people would need to accept that their personal beliefs would not find full expression in their society's political processes. In addition 
to the freedoms and opportunities that the citizens of such states enjoy, this engagement offers individuals and groups the chance to express their culture generally, and to influence political substance: the complexity and bias of particular political conceptions will be framed by the society and, in particular, by those engaging in the dialogue. Through this, individuals, groups and cultures can influence the way that particular issues are understood and treated within a society.

The second example of liberal adherence might be described as the moral liberal, for whom liberalism is accepted as setting out an appropriate political and personal ethic. Such an adherent would be characterised, not as someone who pragmatically separates their political from their personal lives, but as someone who merges both based on their liberal ideals. This would entail adopting principles such as an openness towards and respect for others, and a powerful belief in the rights of people not to be interfered with or harmed. Finally, it might also suggest an ironism, whereby the liberal person recognises uncertainty, or the burdens of judgment, and incorporates this into their structure of beliefs. This example suggests a deeper coherence with and potential engagement in a liberal public culture and political dialogue, but, contained within it, is the belief of furthering the genuine inclusion of others in that process. It is this person who engages more readily with the liberal conceptions on which social institutions are founded, but also is ready to respect others whose relationship to these conceptions are less strongly forged.

The idea of different structural forms of liberal inclusion has no parallel in contemporary liberal theory, but is a natural consequence of diversity and complexity. It allows liberalism to be seen as both political and ethical, and that these do not necessitate a conflict: a single Western society could readily incorporate both. These two examples are not meant to set up two paradigms of liberal adherence, but, rather, represent two focal points within a complex diversity; they are useful focal points to draw from because they represent imagined extremes. The result is a liberal theory that is inclusive and just, and is more easily applied once the universal aims of liberal theory are dropped, and so its focus no longer needs to be as singular. 


\subsection{Some objections}

This framework, and some of the subsequent ideas of an allied liberal conception, has not been presented as a positive structure to the same extent as those works that have been explored throughout this thesis. Despite this, these ideas have been informed by the shortcomings of liberal theory with regard to liberal uncertainty. For this reason, there is enough content here to at least sketch out how the framework could respond to many of the criticisms levelled at contemporary liberalism in this thesis.

The central relationship of the liberal framework is between belief and theory. The former, it is asserted, amounts to a terrain or landscape onto which a liberal conception of justice can be mapped and built. The characterisation of belief as terrain is potentially problematic in two ways. Firstly, it makes liberal theory too reliant on what beliefs actually are. As was mentioned above, this result is disturbing, but is a valid approach given the exhaustion of other foundational approaches. It should also be remembered that this belief is the result of our moral capacities, and is the complex result of historical and social conditions, not to mention the relationship with moral and political theory. The second point is that if belief is merely the terrain, then it is uncertain how to get from that belief to a valid liberal conception. Of course, the idea is that there is no necessity in the relationship between belief and any particular conception; the reason for using the terrain-structure is to offer a ready understanding of this point. A conception arises from existing liberal belief and thought, but how this is constructed depends on the particular person or group who is engaging in the exercise. The mutuality of this account, the importance of both the foundational belief and the process of construction itself, is the essential feature of the relationship. These two difficulties, then, really the observations that it is problematic if theory is either too closely aligned to, or too independent from, belief, and do recognise genuine concerns about the approach. But the impetus for the liberal framework is from the difficulties moral uncertainty reveal about contemporary approaches to liberal theory. These would be difficult to overcome absolutely, and the framework presented here does not attempt this. Rather, it is supposed to represent a coherent response; one that sets out a plausible foundational structure that recognises the value of both belief and theory without finding moral ascendancy in either. It's a necessary consequence of 
this that there will be no single standard to apply to liberal theory, and no direct way of deriving liberal content from an agreed set of facts.

Another criticism, and one that strikes at the heart of the framework and requires more elaboration, is its status as a two levelled liberal conception: that is open in relation to the liberal conceptions that it supports, and yet is closed according to the structure and content of the framework itself. Since this criticism has been made of various other conceptions, it is important for it to be answered here. In particular, the focus should be on the status of the framework itself: its justification and its potentially universal aims. My response is that the framework itself sets out a metaethical position that is not presumed to be universal, and that it contains substantive liberal content that is admitted to be clearly morally contestable. In relation to the first of these points, it presents a characterisation of the relationship between moral and political belief and liberal theory that is supposed to be fundamental, but is not supposed to exclude other possible characterisations, or positions that supplant this relationship with other systems or ideas.

The framework, then, is intended as a positive model of the nature of liberal theory that incorporates content from social values and theory, and has an affinity with liberalism. It is admittedly contestable and is conceived as an expression of theory, rather than as revealing a truth beyond theory. Yet the need for the justification of this approach remains; there must be some process of reasoning by which it can be shown to be valuable. And this justification itself must not contravene its own principles and ideas. Just as a liberal conception, on this approach, is founded on and perceived according to liberal belief, this liberal framework is to be apprised by those theorists and commentators who work and interact within its scope. Its justification, then, rests on its coherence to other beliefs, and the appreciation of it as being valuable. In this way, the content of its justification is that it models liberal theory in a recognisable and a plausible way, and that it is a functional and useful framework out of which to conceive and present conceptions of liberal theory. In particular, it frees that theory from its universal foundations, without removing its nature or power as theory.

This approach to justification, it will be noticed, implies at a level once removed, a model of by which such frameworks can be appreciated and judged. It might be 
asserted that if the framework itself is contestable, then it is this level, the level of justification of such devices, that is universal and fixed. But the approach is admittedly non-foundational in that sense, meaning that just as it permits alternative approaches, it also permits alternative methods of judging such approaches. It does not present an unassailable foundational system, but rather an approach that is recognisable on its own terms, which are the terms of moral uncertainty. This approach is wholly contextual, but not so as to suffer from the same problem as some recent contextualised liberal theories, namely attempting to derive singularity from a diverse pluralism and difference of belief. The view of uncertainty that has prompted this approach would stress the difficulty of ever abstracting to a level beyond context to make an assertion of value. The approach itself, then, is a self-conscious expression of the limitations implied by this uncertainty, but ultimately does not comment on that nature or value of this outside of its own theoretical structure. The hope is that a movement in this direction might reflect a richer paradigm of liberal political theory; richer through being one that is admittedly linked to the context of its perception and understanding.

The liberal framework responds well to these criticisms from uncertainty, as would be expected, since it is constructed as an appropriate response to the same. The point is that such an approach has considerable benefits beyond the nature of this response. Of course, there are other criticisms that might be levelled at it from outside this perspective. In particular, the approach would admit to being without the foundations that some would believe is necessary for a valid moral theory, and consequently, to its liberal content being underdefined. But it would make no apology for these shortcomings, since they are its explicit points of difference from much of contemporary liberal theory, and the features by which it claims value as a different approach. While it would admit these shortcomings, it also represents a challenge to these positions: that, if it is too theoretically open and permissive, then criticism on these grounds should identify how a more closed and definite approach to liberal theory might work, one that represents a plausible response to moral uncertainty.

\subsection{Concluding remarks}


The essence of the approach to liberalism presented here relates liberal theory to belief without reducing theory to belief, retaining both the moral and theoretical characters of this liberalism. This is achieved in two ways. Firstly, by recharacterising the relationship between belief and theory, by applying the metaphor of terrain and structure. This is supposed to supplant the idea implicit in most contemporary forms of liberalism, as to the directness of the link between a liberal conception and its foundations. Secondly, the role of theory is recast from the revelation of truth to being rhetorical, and affecting belief. This adds to theory a flavour of persuasion that on most readings is not currently present, but that seems to assist in describing how theory actually interacts with its readers. This approach may mean that theory loses its sheen of absoluteness or universality, but it also means a more realistic conception of liberal theory, as a text with which its readers engage and interact. Of course, the rhetorical aspect of theory is not supposed to dictate its content and nature; more generally, the separation between belief and theory must be maintained for the approach tot be workable. The nature of this separation is one of the difficult aspects of the liberal framework, and has been explained here in only a cursory way.

As well as the general approach, I suggested a number of more substantive liberal ideas. These are to a large extent suggested by the weight of recent liberal theory, and are informed by the framework itself. They concern the role of dialogue, majoritarianism and bias, the relationship between self and society, and variation in legitimate forms of liberal adherence to a conception. These ideas stem from values that are prominent in post-communitarian liberalism, namely difference and diversity, and conflict and coexistence. The substantive ideas also trade on the links between uncertainty and liberalism, and on the attitude of openness suggested by the framework.

The idea of this framework is that by freeing liberal theory from the need to be derived from clear, albeit potentially contextual, foundations, and to assert a universal effect. This approach denies neither the moral value of liberalism, nor the importance of theory in structuring and explaining it. It is conceived as being useful for the current moment: that liberal theory has shifted to largely difference-based conception, while the uncertainty that this reflects is not mirrored by its approach to justification. 
It is not fully expressed here and, of course, the point is that this might allow liberal theory to develop in a way that is not predicted or predictable. In the context of difference and disagreement, the power of liberalism is that it embraces other doctrines, yet does not pretend to be any less of a doctrine itself. As such, it should seek to be genuinely open, liberal and self-transforming. My contention is that this can be better achieved by it being self-consciously grounded in its own understanding, not as the whole of justice, or even as its best expression, but as an expression of justice that we are able to recognise and appreciate and develop. 


\section{REFERENCES}

\section{Table of books}

Ackerman, B A, Social Justice in the Liberal State (New Haven: Yale University Press, 1980).

Barry, B M, A Treatise on Social Justice (Berkeley: University of California Press, 1989).

Barry, B M, Essays in Political Theory (Oxford: Oxford University Press, 1991).

Barry, B M, Culture and Equality: an egalitarian critique of multiculturalism (Cambridge: Harvard University Press, 2001).

Berlin I, Concepts and Categories: Philosophical Essays (London: Hogarth Press, 1978).

Berlin, I, The Proper Study of Mankind: An Anthology of Essays (London: Pimlico, 1998).

Bertens, J W, The Idea of the Postmodern: A History (London: Routledge, 1995).

Bramsted, E K \& Melhuish, K J (eds.), Western Liberalism: A History of Documents from Locke to Croce (London: Longman, 1978).

Burley, J, Dworkin and his Critics: With Replies by Dworkin (Malden: Blackwell Publishing, 2004).

Daniels, N, Reading Rawls: Critical Studies on Rawls' 'A Theory of Justice (Stanford: Stanford University Press, 1989)

Davion, V \& Wolf, C (eds.), The Idea of a Political Liberalism: essays on Rawls (Lanham: Rowman \& Littlefield, 2000).

Dworkin, R M, Taking Rights Seriously (London: Duckworth, 1978).

Dworkin, R M, A Matter of Principle (Cambridge: Harvard University Press, 1985).

Dworkin, R M, Sovereign Virtue: The Theory and Practice of Equality (Cambridge: Harvard University Press, 2000).

Freeman, S (ed.), John Rawls: Collected Papers (Cambridge: Harvard University Press, 1999)

Galston, W A, Liberal Pluralism: The Implications of Value Pluralism for Political Theory and Practice (Cambridge: Cambridge University Press, 2002). 
Gaus, G F, Justificatory Liberalism: An Essay on Epistemology and Political Theory (New York: Oxford University Press, 1996).

Gaus, G F, Contemporary Theories of Liberalism: Public Reason as a PostEnlightenment Project (London: Sage Publications, 2003).

Gellner, E, Reason and Culture (Oxford: Blackwell Publishers, 1992).

Gellner, E, Conditions of Liberty: Civil Society and its Rivals (New York: Allen Lane, 1994).

Geras, N, Solidarity in the Conversation of Humankind: The Ungroundable Liberalism of Richard Rorty (Guildford: Biddles, 1995).

Gray, J, Liberalisms (London: Routledge, 1989).

Gray, J, Post-Liberalism: Studies in political thought (Suffolk: Richard Clay, 1993).

Gray, J, Enlightenment's Wake: Politics and culture at the close of the modern age (London: Routledge, 1995).

Gray, J, Two Faces of Liberalism (New York: The New Press, 2000).

Gutmann, A \& Thompson, D, Democracy and Disagreement (Cambridge: Harvard University Press, 1996).

Hampshire, S (ed.) Public and Private Morality (Cambridge: Cambridge University Press, 1978).

Hardin, R, Liberalism, Constitutionalism, and Democracy (Oxford: Oxford University Press, 1999).

Honderich, T (ed.), The Oxford Companion to Philosophy (Oxford: Oxford University Press, 1995).

Kekes, J, The Morality of Pluralism (Princeton: Princeton University Press, 1993).

Kekes, J, Against Liberalism (New York: Cornell University Press, 1997).

Kerferd, G B, The Sophistic Movement (Cambridge: Cambridge University Press, 1981).

Kolenda, K, Rorty's Humanistic Pragmatism: Philosophy democratised (Tampa: University of South Florida Press, 1990).

Kymlicka, W, Multicultural Citizenship: A Liberal Theory of Minority Rights (Oxford: Oxford University Press, 1995). 
Kymlicka, W, Politics in the Vernacular: Nationalism, Multiculturalism, and Citizenship (Oxford, Oxford University Press, 2001).

Kymlicka, W, Contemporary Political Philosophy (Oxford: Oxford University Press, second edition, 2002).

Larmore, C E, Patterns of Moral Complexity, (Cambridge: Cambridge University Press, 1987).

Larmore, C E, The Morals of Modernity (Cambridge: Cambridge University Press, 1996).

Lyotard, J F, The postmodern condition: a report on knowledge (Minneapolis:

Minnesota Press, 1984).

MacIntyre, A C, After Virtue (Notre Dame: University of Notre Dame Press, second edition, 1984).

MacIntyre, A C, Whose Justice? Which Rationality? (Notre Dame: University of Notre Dame Press, 1988).

Mautner, T (ed.), The Penguin Dictionary of Philosophy (London, Penguin Books, 2000).

Merquior, J G, Liberalism, Old and New, (Boaton: Tawyne Publishers, 1991).

Mulhall, S \& Swift, A, Liberals and Communitarians (Oxford: Blackwell Publishing, second edition, 1996).

Nozick, R, Anarchy, State, and Utopia (Basic Books, 1974).

Parekh, B C, Rethinking Multiculturalism: Cultural Diversity and Political Theory (Cambridge: Harvard University Press, 2000).

Paul, J (ed), Reading Nozick: Essays on Anarchy, State, and Utopia (Totowa: Rowman \& Littlefield, 1981).

Porter, R, Enlightenment (London: Penguin, 2000).

Rawls, J, A Theory of Justice (Cambridge: Harvard University Press, Revised edition, 1999)

Rawls, J, Political Liberalism (New York: Columbia University Press, paperback edition, 1996).

Rawls, J \& Kelly, E, Justice as Fairness: A Restatement (Cambridge, Harvard University Press, 2001).

Raz, J, The Morality of Freedom (Oxford: Clarendon Press, 1986). 
Raz, J, Ethics in the Public Domain: Essays in the morality of law and politics (Oxford: Clarendon Press, 1994).

Rorty, R, Contingency, Irony, and Solidarity (Cambridge: Cambridge University Press, 1989).

Rorty, R, Objectivity, Relativism, and Truth (Cambridge: Cambridge University Press, 1991).

Rorty, R, Philosophy and Social Hope (London: Penguin Books, 1999).

Russell, B A W, Religion and Science (Oxford: Oxford University Press, 1997).

Sandel, M J, Liberalism and the Limits of Justice (Cambridge: Cambridge University Press, second edition, 1998).

Sen, A K, Inequality Reexamined (Cambridge: Harvard University Press, 1992).

Tan, K-C, Toleration, Diversity, and Global Justice (Pennsylvania: Pennsylvania State University Press, 2000).

Tarnas, R, The Passion of the Western Mind: Understanding the Ideas That Have Shaped Our World View (London: Pimlico, 1996).

Taylor, C, Sources of the Self: The making of the modern identity (Cambridge: Harvard University Press, 1989).

Taylor, C, Multiculturalism: Examining the Politics of Recognition (Princeton: Princeton University Press, 1994).

Tully, J, Strange Multiplicity: Constitutionalism in an age of diversity (Cambridge: Cambridge University Press, 1995).

Walzer, M, Spheres of Justice: A Defence of Pluralism and Equality (USA: Basic Books, 1983). 


\section{Table of articles}

M Akan, 'Contextualizing Multiculturalism' (2003) 38(2) Studies in Comparative International Development 57.

L A Alexander 'Liberalism as Neutral Dialogue: Man and Manna in the Liberal State' (1981) 28 UCLA Law Review 816.

C W Anderson, 'Pragmatism and Liberalism, Rationalism and Irrationalism: A Response to Richard Rorty' (1991) 23(3) Polity 257.

E S Anderson, 'What's the Point of Equality?' (1999) 109(2) Ethics 287.

R Arneson, 'Against 'Complex' Equality' in Millerm D \&Walzer, M (eds), Pluralism, Justice and Equality (Oxford: Oxford University Press, 1995).

R J Bernstein, 'One Step Forward, Two Steps Backward: Richard Rorty on Liberal Democracy and Philosophy' (1987) 15(4) Political Theory 538.

J W Boettcher, 'What is Reasonableness?' (2004) 30(5-6) Philosophy and Social Criticism 597.

J Bohman, 'Public Reason and Cultural Pluralism: Political Liberalism and the Problem of Moral Conflict' (1995) 23(3) Political Theory 253.

J W Chapman, 'Rawls's Theory of Justice' 69 (1975) American Political Science Review, 588.

M Clayton, 'Liberal Equality and Ethics' (2002) 113(1) Ethics 8.

G Crowder, 'John Gray's Pluralist Critique of Liberalism' (1998) 15(3) Journal of Applied Philosophy 287.

G Crowder, 'Two Value-pluralist Arguments for Liberalism' (2002) 37(3) Australian Journal of Political Science 457.

W M Curtis, 'Liberals and Pluralists: Charles Taylor vs. John Gray' (2007) 6 Contemporary Political Theory 86.

G Den Hartogh, 'The Architectonic of Michael Walzer's Theory of Justice' (1999) 27(4) Political Theory 491.

G Doppelt, 'Is There a Multicultural Liberalism?' (1997) 41(2) Inquiry 223.

R M Dworkin, 'What Liberalism Isn't' (1983) 29 New York Review of Books 47.

R M Dworkin, 'Sovereign Virtue Revisited' (2002) 113(1) Ethics 106. 
D Estlund, 'The Insularity of the Reasonable: Why Political Liberalism Must Admit the Truth' (1998) 108 Ethics 252.

M Evans, 'Is public justification central to liberalism?' (1999) 4(1) Journal of Political Ideologies 117.

J M O’Fallon, ‘The Meanings of Justice' (1983) 62 Texas Law Review, 763.

J Fennell, 'Harry Neumann and the Political Piety of Rorty's Postmodernism' (1999) 26(2) Interpretation 257.

J S Fishkin, 'Can There Be a Neutral Theory of Justice?' (1983) 93(2) Ethics 348.

J S Fishkin, Book Review: Patterns of Moral Complexity by Charles Larmore, (1989) 17(1) Political Theory 153.

M Fleurbaey, 'Equality of Resources Revisited' (2002) 13(1) Ethics 82.

P D Forster, 'Problems with Rorty's Pragmatist Defence of Liberalism' (2000) 25 Journal of Philosophical Research 345.

B H Fried, 'Begging the Question with Style: Anarchy, State, and Utopia at Thirty Years' 23(1) (2005) Social Philosophy and Policy 221.

W A Galston, 'Defending Liberalism' (1982) 76(3) American Political Science Review 621.

W A Galston, 'Community, Democracy, Philosophy: The Political Thought of Michael Walzer' (1989) 17(1) Political Theory 119.

W A Galston, 'Liberal Pluralism: A reply to Talisse' (2003) 3 Contemporary Political Theory 140.

R D Gastil, 'Beyond a Theory of Justice' (1975) 85(3) Ethnopsychologie 183.

G F Gaus, 'Reasonable Pluralism and the Domain of the Political: How the Weaknesses of John Rawls's Political Liberalism Can be Overcome by a Justificatory Liberalism' (1999) 42 Inquiry 259.

R P George, 'The Unorthodox Liberalism of Joseph Raz' (1991) 53 The Review of Politics 652.

A Gutmann, 'The Central Role of Rawls’ Theory’ (1989) 36(3) Dissent 338.

A Gutmann, 'Liberty and Pluralism in Pursuit of the Non-Ideal' (1999) 66(4) Social Research 1039.

H Haber, 'Richard Rorty's failed politics' (1993) 7(1) Social Epistemology 61.

R M Hare, 'Rawls’ Theory of Justice I' (1973) Philosophical Quarterly 144. 
R M Hare, 'Rawls’ Theory of Justice II' (1973) Philosophical Quarterly 241.

J C Harsanyi, 'Can the Maximin Principle Serve as a Basis for Morality? A Critique of John Rawls's Theory' (1975) 69 American Political Science Review 594.

S Hendley, 'Putting ourselves up for question: A postmodern critique of Richard Rorty's postmodern bourgeois liberalism' (1995) 29 The Journal of Value Inquiry 241.

K Hyams, 'Nozick's Real Argument for the Minimal State' (2004) 12(3) The Journal of Political Philosophy 353.

C Joppke, 'Multicultural Citizenship: A Critique' (2001) 42(2) European Journal of Sociology 431.

A Kaufman, 'The myth of the patterned principle: Rawls, Nozick and entitlements' (2004) 36(4) Polity 559.

C Knight, 'Liberal Multiculturalism Reconsidered' (2004) 24(3) Politics 189.

C Kukathas, 'Liberalism and Multiculturalism: The Politics of Indifference' (1998) 26(5) Political Theory 686.

C Kukathas, 'Multiculturalism as Fairness: Will Kymlicka's Multicultural Citizenship' (1997) 5(4) Journal of Political Philosophy 406.

W Kymlicka, 'Rawls on Teleology and Deontology' (1988) 17(3) Philosophy and Public Affairs 173.

W Kymlicka, 'Liberalism, dialogue and multiculturalism' (2001) 1(1) Ethnicities 128.

A J Langlois, 'Redescribing Human Rights' (1998) 27(1) Millennium: Journal of International Studies 1.

C E Larmore, 'Respect for Persons' (2005) 7.2 The Hedgehog Review 66.

M H Lessnoff, 'Justice, Social Contract and Universal Prescription' 28 (1978) Philosophical Quarterly 65.

P Lom, 'East Meets West - Jan Patocka and Richard Rorty on Freedom' (1999) 27(4) Political Theory 447.

D McCabe, 'Joseph Raz and the Contextual Argument for Liberal Perfectionism' (2001) 111 Ethics 493.

T McCarthy, 'Kantian Constructivism and Reconstructivism: Rawls and Habermas in Dialogue' (1994) 105 Ethics 44. 
J McNaughton, 'John Rawls and the Conflict Between Right and Good' (1980) Auslegung 270.

S Macedo, 'The Politics of Justification' (1990) 18(2) Political Theory 280.

G M Mara \& S L Dovi, 'Mill, Nietzsche, and the Identity of Postmodern Liberalism' (1995) 57(1) The Journal of Politics 1.

P A Michelbach et al., 'Doing Rawls Justice: An Experimental Study of Income Distribution Norms' (2003) 47(3) American Journal of Political Science 523.

L S Mullenix, 'The Limits of "Complex Equality"' (1984) 97(4) Harvard Law Review, 1801.

D D Murphey, “"Multiculturalism” and the West' (2005) 30(2) Journal of Social, Political, and Economic Studies 203.

P Neal, 'Liberalism \& Neutrality’ (1985) 17(4) Polity 664.

P Neal, 'Perfectionism with a Liberal Face? Nervous Liberals and Raz's Political Theory' (1994) 20(1) Social Theory and Practice 25.

P Neal, 'Three readings of political liberalism: Rawls, Maritain and Crick' (2000)

5(2) Journal of Political Ideologies 225.

J M O’Fallon, ‘The Meanings of Justice' (1983) 62 Texas Law Review 763.

M Otsuka, 'Luck, Insurance and Equality' (2002) 113(1) Ethics 40.

B C Parekh, 'A response' (2001) 1(1) Ethnicities 137.

C Perrin, Book review: Strange Multiplicity: Constitutionalism in an Age of Diversity by James Tully (1999) 10(2) European Journal of International Law 468.

J Raz ‘Facing Up: A Reply’ (1988) 62 Southern California Law Review 1153.

J Raz, 'Facing Diversity: The Case of Epistemic Abstinence' (1990) 19(1) Philosophy and Public Affairs 3.

D H Regan, 'Authority and Value: Reflections on Raz's Morality of Freedom' (1988) 62 Southern California Law Review 995.

S Rennie, 'Elegant Variations: remarks on Rorty's 'Liberal Utopia' (1998) 17(4) South African Journal of Philosophy 313.

R Rorty, 'Thugs and Terrorists: A Reply to Bernstein' (1987) 15(4) Political Theory 564.

H Ryu, 'Ethics of Ambiguity and Irony: Jacques Derrida and Richard Rorty' (2001) 24 Human Studies 5. 
S Scheffler, 'What is Egalitarianism?' (2003) 31(1) Philosophy and Public Affairs 4.

D Scott, 'Culture in Political Theory’ (2003) 31(1) Political Theory 92.

J Seglow, 'Neutrality and Equal Respect: On Charles Larmore's Theory of Political Liberalism' (2003) 37 The Journal of Value Inquiry 83.

A Stark, 'Beyond Choice: Rethinking the Post-Rawlsian Debate over Egalitarian Justice' (2002) 30(1) Political Theory 36.

R B Talisse, 'Two-faced liberalism - John Gray's pluralist politics and the reinstatement of enlightenment liberalism' (2000) 14(4) Critical Review 441.

R B Talisse, 'Can Value Pluralists be Comprehensive Liberals? Galston's Liberal Pluralism' (2004) 3 Contemporary Political Theory 127.

R B Thigpin \& L A Downing, 'Liberalism and the Neutrality Principle' (1983) 11(4) Political Theory 585.

K Topper, 'Richard Rorty, Liberalism and the Politics of Redescription' (1995) 89(4) American Political Science Review 954.

R van der Veen, 'Equality of Talent Resources: Procedures or Outcomes?' (2002) 113(1) Ethics 55.

J Waldron, 'Theoretical Foundations of Liberalism' (1987) 37 The Philosophical Quarterly 127.

J Waldron, 'Autonomy and Perfectionism in Raz's Morality of Freedom' (1988) 62 Southern California Law Review 1097.

D M Weinstock, Book review: Democracy and Disagreement by Amy Gutmann \& Dennis Thompson (1997) 91(3) The American Political Science Review 724.

B Williams, 'Space Talk: The Conversation Continued' (1983) 93(2) Ethics 367.

L Wenar, 'Political Liberalism: An Internal Critique' (1995) 106 Ethics 32.

B Yack, 'Multiculturalism and the Political Theorists' (2002) 1 European Journal of Political Theory 107. 